\title{
Modeling of Monostatic Bottom Backscattering from Three-Dimensional Volume Inhomogeneities and Comparisons with Experimental Data
}

\author{
by \\ Dan Li \\ B. S., Electrical Engineering \\ University of Science and Technology of China (1992) \\ M. S., O. E., Ocean Engineering \\ M.I.T. / Woods Hole Oceanographic Institution (1995) \\ Submitted in partial fulfillment of the \\ requirements for the degree of \\ DOCTOR OF PHILOSOPHY IN OCEANOGRAPHIC ENGINEERING \\ at the \\ MASSACHUSETTS INSTITUTE OF TECHNOLOGY \\ and the \\ WOODS HOLE OCEANOGRAPHIC INSTITUTION \\ September 1997 \\ (C) Dan Li, 1997. All rights reserved.
}

The author hereby grants to MIT and WHOI permission to reproduce and to distribute copies of this thesis document in whole or in part.

Signature of Author

Department of Ocean Engineering, MIT and the

MIT/WHOI Joint Program in Oceanographịc Engineering

Certified by

$\checkmark$ Dr. George V. Frisk

Senior Scientist, Woods Hole Oceanographic Institution

Thesis Supervisor

Certified by

(J 0 Dr. Dajun Tang

Associate Scientist, Woods Hole Oceanographic Institution

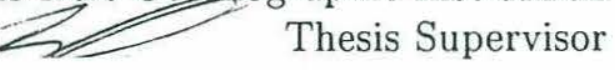

Accepted by

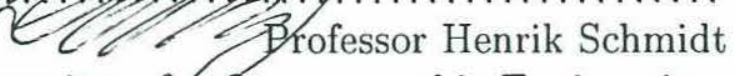

Chairman, Joint Committee for Oceanographic Engineering, Massachusetts Institute of Technology-Woods Hole Oceanographic Institution 


\title{
Modeling of Monostatic Bottom Backscattering from Three-Dimensional Volume Inhomogeneities and Comparisons with Experimental Data
}

\author{
by \\ Dan Li
}

\begin{abstract}
Submitted to the Massachusetts Institute of Technology/ Woods Hole Oceanographic Institution in partial fulfillment of the requirements for the degree of DOCTOR OF PHILOSOPHY IN OCEANOGRAPHIC ENGINEERING
\end{abstract}

\begin{abstract}
Acoustic propagation in the ocean inevitably encounters inhomogeneities of various types, which give rise to scattering. Acoustic scattering from rough water/bottom interfaces comprised of exposed rocks and sea mountains gives way to volumetric scattering in areas with flat interfaces and thick sediment cover. The data analysis of the ARSRP backscattering experiment revealed that random inhomogeneities in two irregular layers beneath the seafloor were the primary contributors to oblique backscattering in a sediment pond on the western flank of the Mid-Atlantic Ridge. In this thesis, an attempt has been made to model monostatic backscattering from 3-D volume inhomogeneities in the sediment and to compare the results with the ARSRP backscattering data.

A scattering process cannot be modeled correctly without a proper account of the incident field. Several approximate propagation models have been evaluated against the exact solution, while the appropriateness of using the equivalent surface scattering strength in volume scattering characterizations is studied. This study concludes that precautions need to be taken in modeling both the propagation effects and the scattering mechanisms associated with the bottom volume scattering process.

A volume scattering model based on perturbation theory and the Born approximation is developed incorporating contributions from both sound speed and density fluctuations. With the propagation part handled accurately by OASES and random fluctuations generated effectively by a new scheme modified from the spectral method, the model is capable of simulating the monostatic backscattered field and time series due to 3-D volumetric sediment inhomogeneities. Both the characteristic length scale and power spectrum descriptions of the random inhomogeneities are shown to have great impact on the backscattered field by parameter studies in a free-space scenario. The important roles played by horizontal anisotropy and the vertical correlation of the random field have been demonstrated. Density fluctuations are further confirmed to be the dominant force in backscattering. The model matches the ARSRP backscattering data very well, with the fluctuations of sound speed and density in the two irregular layers described by a power law type of power spectrum.
\end{abstract}


Thesis Supervisor: Dr. George V. Frisk

Senior Scientist

Woods Hole Oceanographic Institution

Thesis Supervisor: Dr. Dajun Tang

Associate Scientist

Woods Hole Oceanographic Institution 


\section{Acknowledgments}

I am extremely grateful to my thesis advisors Dr. George Frisk and Dr. Dajun Tang. Without their continuous encouragement, enormous help and insightful guidance, it would have been impossible for me to finish this thesis work. I will always remember George's famous mark "clarify" and D.J.'s back-to-the-simplest philosophy.

My indebtedness goes to my other committee members, Prof. Henrik Schmidt and Dr. James Presig as well. Henrik has always provided with excellent comments on my work and his OASES code is an important part of my scattering model. And the good advice given by Dr. James Presig on array signal processing was such a great help in the beginning of the data analysis. Thanks also goes to Dr. Jim Lynch

for chairing my defense and Dr. Dezhang Chu for the wonderful discussions and good suggestions.

I would like to express my gratitude to my academic advisor at MIT, Prof. Arthur Baggeroer, and all the MIT/WHOI Joint Program staffs for their support. I really appreciate the help of Cynthia Sellers in all these years and I will certainly miss the food prepared by Sabina.

To my fellow-students in 5-007 and 5-435, thank you for the wonderful atmosphere that you have created and the team-work spirit that I have enjoyed. Believe me, you are the best.

Life would not have been so fun without all my friends. Qing, Henry, and Chenyang, how about playing some Tetris the next time we get together? And thank you for introducing me to the "real world". Wenjie and Co., guess no card game is scheduled for now. Thank you, Elaine, for many "Sloanish" advice, Liang-wu, for the ride to chase the sunset, Richard, for the parties, and Feng, for making me a part-time Ashdowner. Helen, Lan and Xiaoou, I will surely not forget the summer at Cape Cod.

My most grateful thanks goes to my parents and my sister. You are always in my heart. 


\section{Contents}

1 Introduction $\quad 17$

1.1 General Background . . . . . . . . . . . . . . . . 17

1.2 Data Analysis Results of ARSRP Backscattering Experiment . . . . . 19

1.3 Objective and Approach . . . . . . . . . . . . . . 25

1.4 Literature Review . . . . . . . . . . . . . . . . . . . 26

1.5 Contributions ......................... 34

1.6 Overview of the Thesis . . . . . . . . . . . . 35

2 Evaluation of Sound Propagation Models Used in Bottom Volume $\begin{array}{ll}\text { Scattering Studies } & 37\end{array}$

2.1 Statement of the Problem . . . . . . . . . . . . . . . 38

2.2 The Scattering Cross Section . . . . . . . . . . . . . . . . . . . . . . . 39

2.3 Bottom Propagation Models . . . . . . . . . . . . . . . . . 45

2.4 Comparison of Propagation Models . . . . . . . . . . . . . . . 52

2.4 .1 Two Isovelocity Half-Spaces . . . . . . . . . . . . . . . . 54

2.4.2 Two Half-Spaces with Sound Speed Gradient in the Lower Half 63

2.4.3 Three Layers with a Sound Speed Gradient in the Middle Layer 72

2.5 Summary . . . . . . . . . . . . . . . . . . 75

3 Formulations for Modeling Bottom Volume Backscattering $\quad 77$

3.1 Volume Scattering Theory . . . . . . . . . . . . . . . 78

3.2 Correlation Functions of Sound Speed Variations . . . . . . . . . . . . 82 
3.3 Backscattering from Sound Speed Variations in the Free-Space Scenario: Analytical Solutions . . . . . . . . . . . . . . . . . . . 88

3.3.1 Backscattering from 2-D Isotropic Random Field . . . . . . 91

3.3.2 Backscattering from 2-D Anisotropic Random Field . . . . . 95

3.3.3 Effect of the Vertical Correlation . . . . . . . . . . . 98

4 Generation of Azimuthally-Summed 3-D Random Sound Speed Vari$\begin{array}{ll}\text { ations } & 102\end{array}$

4.1 Statement of the Problem . . . . . . . . . . . . . . . . . . . . 102

4.2 Formulas . . . . . . . . . . . . . . . . . . . . . . . . . 104

4.3 Numerical Examples . . . . . . . . . . . . . . . . . . . . . . . 109

$4.3 .1 \quad$ Isotropic Case . . . . . . . . . . . . . . . . . . 110

4.3 .2 Anisotropic Case . . . . . . . . . . . . . . . . . . . . . 113

4.4 Summary . . . . . . . . . . . . . . . . . . . . 117

5 Numerical Simulations of Backscattered Time Series 118

5.1 Numerical Simulations . . . . . . . . . . . . . . . . . . . . . . . . . 119

5.2 Comparisons with Analytic Solutions in the Free-Space Scenario . . . 122

$5.2 .1 \quad$ 2-D Isotropic Case . . . . . . . . . . . . . . . . 125

$5.2 .2 \quad$ 2-D Anisotropic Case . . . . . . . . . . . . . . . . . . 129

5.2.3 3-D Vertically Correlated Case . . . . . . . . . . . . . . . . . 134

5.3 The Effect of Density Variations . . . . . . . . . . . . . 136

6 Comparison of Model Results and ARSRP Backscattering Data 139

6.1 Experiment Description . . . . . . . . . . . . . . . . . . 140

6.2 Data Processing . . . . . . . . . . . . . . . . . . . 147

6.3 Selection of Model Parameters and Estimation of the Backscattering Strength . . . . . . . . . . . . . . . . . 150

6.4 Model and Data Comparison . . . . . . . . . . . . . . . . . 160

7 Conclusions and Future Work 168

7.1 Conclusions . . . . . . . . . . . . . . . . . . . 168 
7.2 Future Work . . . . . . . . . . . . . . . . . . . . . 171

A The Variance and Power Spectral Density of the 2-D AzimuthallySummed Random Field with Gaussian Correlation Function 172

B Estimation of the Standard Deviation $\quad 175$ 


\section{List of Figures}

1-1 The inferred sediment structure at the east side of the sediment pond. 20

1-2 The average envelope of oblique angle beamforming results over 8 pings at the east side of the sediment pond (arbitrary units). . . . . . . 22

1-3 The inferred sediment structure at the west side of the sediment pond. 23

1-4 The average envelope of oblique angle beamforming results over 8 pings at the west side of the sediment pond (arbitrary units). . . . . . . 24

2-1 Geometry of the classical scattering problem. . . . . . . . . . . 40

2-2 Schematic illustration of the surface and volume scattering coefficient definitions. . . . . . . . . . . . . . . . . . . . 4 41

2-3 Schematic illustration of the equivalent surface scattering coefficient definition. . . . . . . . . . . . . . . . . . . . . 44 43

2-4 The experimental scenario. . . . . . . . . . . . . . . 47

2-5 Schematic of scattering geometry for a two isovelocity half-space case. $\quad 49$

2-6 The sound speed profile for three types of bottom: (a) two isovelocity half spaces; (b) two half spaces with a sound speed gradient in the bottom; (c) three layers with a sound speed gradient in the middle layer. 53

2-7 Comparison of transmission loss in the environment with no critical angle. Parameters are: $f=500 \mathrm{~Hz}, c_{0}=1530 \mathrm{~m} / \mathrm{s}, c_{1}=1510 \mathrm{~m} / \mathrm{s}$, $\rho_{1}=1.72 \mathrm{~g} / \mathrm{cm}^{3}, \delta=0.00164, z_{s}=100 \lambda$. The transmitted field at (a) $z=0 ;$ (b) $z=-2 \lambda \ldots \ldots \ldots \ldots \ldots \ldots \ldots \ldots \ldots$ 
2-8 Comparison of transmission loss with the critical angle effect for a low attenuation bottom. Parameters are: $f=500 \mathrm{~Hz}, c_{0}=1500 \mathrm{~m} / \mathrm{s}$, $c_{1}=1560 \mathrm{~m} / \mathrm{s}, \rho_{1}=1.8 \mathrm{~g} / \mathrm{cm}^{3}, \delta=0.0027, z_{s}=100 \lambda$. The transmitted field at (a) $z=0$; (b) $z=-\lambda$; (c) $z=-2 \lambda \ldots \ldots \ldots \ldots \ldots$

2-9 The detailed comparison. Parameters are the same as that in Fig. 2-8(c). 57

2-10 The critical angle effect for a high attenuation bottom. Parameters are: $f=40000 \mathrm{~Hz}, c_{0}=1500 \mathrm{~m} / \mathrm{s}, c_{1}=1689 \mathrm{~m} / \mathrm{s}, \rho_{1}=1.97 \mathrm{~g} / \mathrm{cm}^{3}$, $\delta=0.0166, z_{s}=100 \lambda$. The transmitted field at (a) $z=0$; (b) $z=-\lambda$; (c) $z=-2 \lambda$.

2-11 The equivalent surface backscattering strength for a low attenuation bottom assuming point scatterers. Parameters are the same as that in Fig. 2-8 and $\sigma_{v}=0.00003$. The backscattering strength and the difference as the scattering layer begins at (a)(b) $z=0 ;(\mathrm{c})(\mathrm{d}) z=-\lambda$;

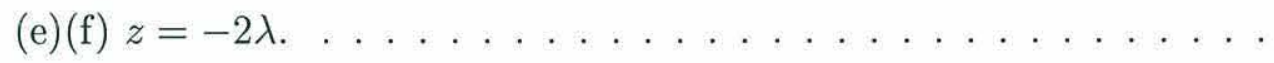

2-12 The equivalent surface backscattering strength for a high attenuation bottom assuming point scatterers. Parameters are the same as that in Fig. 2-10 and $\sigma_{v}=0.000906$. The backscattering strength and the difference as the scattering layer begins at (a)(b) $z=0$; (c)(d) $z=-\lambda$;

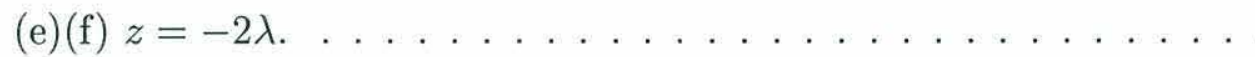

2-13 The frequency dependence of the comparison results. Parameters are: $c_{0}=1530 \mathrm{~m} / \mathrm{s}, c_{1}=1510 \mathrm{~m} / \mathrm{s}, \rho_{1}=1.72 \mathrm{~g} / \mathrm{cm}^{3}, \delta=0.00164, \mathrm{~g}=1 \mathrm{~s}^{-1}$, $z_{s}=100 \lambda, z=0$ and (a) $f=100 \mathrm{~Hz}$; (b) $f=300 \mathrm{~Hz}$; (c) $f=500 \mathrm{~Hz} .64$

2-14 The frequency and depth dependence of the comparison results for a low attenuation bottom with a critical angle. Parameters are $c_{0}=$ $1500 \mathrm{~m} / \mathrm{s}, c_{1}=1560 \mathrm{~m} / \mathrm{s}, \rho_{1}=1.8 \mathrm{~g} / \mathrm{cm}^{3}, \delta=0.0027, \mathrm{~g}=1 \mathrm{~s}^{-1}, z_{s}=$ $100 \lambda$ and (a) $f=100 \mathrm{~Hz}, z=0$; (b) $f=100 \mathrm{~Hz}, z=-2 \lambda$; (c) $f=500 \mathrm{~Hz}, z=0$; (d) $f=500 \mathrm{~Hz}, z=-2 \lambda$; (e) $f=5000 \mathrm{~Hz}$, $z=0$;(f) $f=5000 \mathrm{~Hz}, z=-2 \lambda \ldots \ldots \ldots \ldots \ldots$

2-15 The propagation paths for two half-space fast bottom with an upwardrefracting sound speed profile in the bottom. . . . . . . . . . . . 
2-16 The depth dependence for a high-attenuation bottom. Parameters are: $f=100 \mathrm{~Hz}, c_{0}=1500 \mathrm{~m} / \mathrm{s}, c_{1}=1689 \mathrm{~m} / \mathrm{s}, \rho_{1}=1.97 \mathrm{~g} / \mathrm{cm}^{3}, \delta=$ 0.0166, $g=1 s^{-1}, z_{s}=100 \lambda$. The transmitted field at (a) $z=0$; (b)

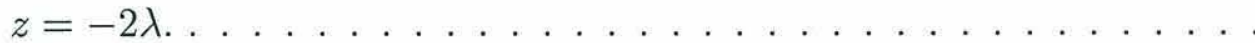

2-17 The sound speed gradient dependence. Parameters are the same as that in Fig. 2-13(a) except the gradient (a) $g=1 s^{-1}$; (b) $g=4 s^{-1}$; (c) $g=10 s^{-1}$.

2-18 The equivalent surface backscattering strength for a high-attenuation bottom assuming point scatterers. Parameters are the same as that in Fig. 2-16 with the starting depth of the scattering layer to be at $z=0$. (a) the backscattering strength; (b) differences.

2-19 The equivalent surface backscattering strength for a low-attenuation bottom assuming point scatterers. Parameters are: $f=2300 \mathrm{~Hz}, c_{0}=$ $1550 \mathrm{~m} / \mathrm{s}, c_{1}=1576 \mathrm{~m} / \mathrm{s}, \rho_{1}=1.83 \mathrm{~g} / \mathrm{cm}^{3}, \delta=0.00193, \mathrm{~g}=10 \mathrm{~s}^{-1}$, $z_{s}=100 \lambda$. The scattering layer starts at $z=0$. (a) the backscattering strength; (b) differences. . . . . . . . . . . . . . . . .

2-20 Figure 15(b) in Mourad and Jackson's paper [1]. The circles here represent the data points. . . . . . . . . . . . . . .

2-21 The middle layer thickness dependence for a three layer environment with a low attenuation middle layer. Parameters are: $f=500 \mathrm{~Hz}$, $c_{0}=1530 \mathrm{~m} / \mathrm{s}, c_{1}=1510 \mathrm{~m} / \mathrm{s}, c_{2}=1800 \mathrm{~m} / \mathrm{s}, \rho_{1}=1.72 \mathrm{~g} / \mathrm{cm}^{3}, \rho_{2}=$ $1.9 \mathrm{~g} / \mathrm{cm}^{3}, \delta_{1}=0.00164, \delta_{2}=0.019, \mathrm{~g}=1 \mathrm{~s}^{-1}, z_{s}=100 \lambda, z=0$ and (a) $H=2 \lambda$; (b) $H=10 \lambda \ldots \ldots \ldots \ldots$

2-22 The middle layer thickness dependence for a three-layer environment with a high attenuation middle layer. Parameters are: $f=100 \mathrm{~Hz}$, $c_{0}=1500 \mathrm{~m} / \mathrm{s}, c_{1}=1689 \mathrm{~m} / \mathrm{s}, c_{2}=1800 \mathrm{~m} / \mathrm{s}, \rho_{1}=1.97 \mathrm{~g} / \mathrm{cm}^{3}, \rho_{2}=$ $2.2 \mathrm{~g} / \mathrm{cm}^{3}, \delta_{1}=0.0166, \delta_{2}=0.019, \mathrm{~g}=4 \mathrm{~s}^{-1}, z_{s}=100 \lambda, z=0$ and (a) $H=2 \lambda$; (b) $H=10 \lambda$. 
3-1 Functional form of the correlation function $G_{\nu}(b)$ plotted for values of $\nu=0,1 / 2$, and $1 \ldots \ldots \ldots \ldots \ldots \ldots \ldots \ldots \ldots \ldots \ldots \ldots \ldots \ldots \ldots$

3-2 Normalized power spectra plotted in log-log space. . . . . . . . . 87

3-3 Normalized isotropic power spectra for different kinds of correlation functions $\left(l_{x}=l_{y}=1 m, \nu=1\right.$ in power law distribution). . . . . 88

3-4 The free-space monostatic backscattering scenario. . . . . . . . . . 89

3-5 Backscattering from sound speed variations with a 2-D isotropic Gaussian correlation function. . . . . . . . . . . . . . . . . . 92

3-6 Backscattering from sound speed variations with a 2-D isotropic exponential correlation function. . . . . . . . . . . . . . . . . . 93

3-7 Backscattering from sound speed variations with a 2-D isotropic Von Karman function as correlation function $(\nu=1) \ldots \ldots \ldots \ldots . . .94$

3-8 Backscattering from sound speed variations with different kinds of 2-D isotropic correlation functions. . . . . . . . . . . . .

3-9 Backscattering from sound speed variations with a 2-D anisotropic Gaussian correlation function. . . . . . . . . . . . 96

3-10 Backscattering from sound speed variations with a 2-D anisotropic exponential correlation function. . . . . . . . . . . . . . .

3-11 Backscattering from sound speed variations with a 2-D anisotropic Von Karman function as the correlation function $(\nu=1) . \ldots \ldots \ldots$

3-12 Backscattering from sound speed variations with an isotropic Gaussian distribution horizontally and an exponential correlation vertically $(\sigma=$ $\left.0.05, l_{x}=l_{y}=2 m, f=400 \mathrm{~Hz}, H=400 m, D=7.5 m\right) \ldots \ldots \ldots$

3-13 Backscattering from sound speed variations with an isotropic exponential distribution horizontally and an exponential correlation vertically $\left(\sigma=0.05, l_{x}=l_{y}=2 m, f=400 \mathrm{~Hz}, H=400 m, D=7.5 m\right) \ldots \ldots$

4-1 10 realizations of azimuthally-summed 2-D random fields with a Gaussian correlation function and $\sigma=1 \%, l_{x}=l_{y}=5 m \ldots \ldots \ldots 111$ 
4-2 Comparison of radial power spectral density estimate with exact radial power spectral density for an ensemble of 100 realizations (isotropic case) 111

4-3 Comparison of variance estimate with exact variance for an ensemble of 100 realizations(isotropic case) . . . . . . . . . . . . . . . 112

4-4 Comparison of radial power spectral density estimate with exact power spectral density for an ensemble of 100 realizations using the FFP

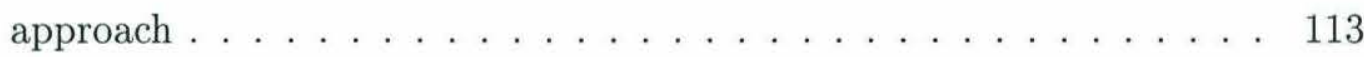

4-5 10 realizations of azimuthally-summed 2-D random fields with a Gaussian correlation function and $\sigma=1 \%, l_{x}=2 m, l_{y}=5 m \ldots . . . .114$

4-6 Comparison of radial power spectral density estimate with exact radial power spectral density for ensemble of 100 realizations (anisotropic case) 115

4-7 Comparison of variance estimate with exact variance for an ensemble of 100 realizations(anisotropic case) . . . . . . . . . . . . . . 116

4-8 Comparison of radial power spectral densities for random fields with the same correlation length $l_{y}$ but different correlation length $l_{x}$. The estimated radial spectral densities are obtained by an ensemble average of 100 realizations . . . . . . . . . . . . . . . 116

5-1 The source signal and its spectrum. . . . . . . . . . . . . 120

5-2 Simulated backscattered returns from an inhomogeneous layer. The correlation function of random sound speed variations within the layer are exponential both horizontally and vertically. . . . . . . . .

5-3 The convergence test for backscattering from 2-D sound speed variations with a power law distribution $\left(l_{x}=l_{y}=1 m\right) . \ldots \ldots 123$

5-4 Backscattering from 2-D isotropic sound speed variations with a Gaussian correlation function, averaging over 50 realizations $(f=400 \mathrm{~Hz}, \mathrm{H}=$

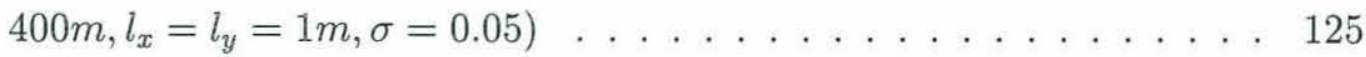


5-5 Comparison of the backscattering strength estimated from the numerically simulated backscattered returns with the analytic solution. Sound speed variations have a Gaussian correlation function. ( $f=$ $\left.400 H z, H=400 m, l_{x}=l_{y}=1 m, \sigma=0.05\right) \ldots \ldots . \ldots 126$

5-6 Backscattering from 2-D isotropic sound speed variations with an exponential correlation function, averaging over 50 realizations $(f=$ $\left.400 H z, H=400 m, l_{x}=l_{y}=0.84 m, \sigma=0.05\right) \ldots \ldots . . . . .$.

5-7 Comparison of the backscattering strength estimated from the numerically simulated backscattered returns with the analytic solution. Sound speed variations have an exponential correlation function. $(f=$ $\left.400 H z, H=400 m, l_{x}=l_{y}=0.84 m, \sigma=0.05\right) \ldots \ldots . . . .$.

5-8 Backscattering from 2-D isotropic sound speed variations with a power law distribution, averaging over 50 realizations $(f=400 \mathrm{~Hz}, \mathrm{H}=$ $\left.400 m, l_{x}=l_{y}=1 m, \sigma=0.05, \nu=1\right) \ldots \ldots \ldots$

5-9 Comparison of the backscattering strength estimated from the numerical simulated backscattering returns with the analytic solution. Sound speed variations have a power law distribution. $(f=400 \mathrm{~Hz}, \mathrm{H}=$ $\left.400 m, l_{x}=l_{y}=1 m, \sigma=0.05, \nu=1\right) \ldots \ldots \ldots$

5-10 Backscattering from 2-D anisotropic sound speed variations with a Gaussian distribution, averaging over 50 realizations ( $f=400 \mathrm{~Hz}, \mathrm{H}=$ $\left.400 m, l_{x}=1 m, l_{y}=0.6 m, \sigma=0.05\right) \ldots \ldots \ldots$

5-11 Comparison of the backscattering strength estimated from the numerically simulated backscattered returns with the analytic solution. Sound speed variations have a Gaussian distribution. ( $f=400 \mathrm{~Hz}, \mathrm{H}=$ $\left.400 m, l_{x}=1 m, l_{y}=0.6 m, \sigma=0.05\right) \ldots \ldots . \ldots \ldots$

5-12 Backscattering from 2-D anisotropic sound speed variations with an exponential distribution, averaging over 50 realizations $(f=400 \mathrm{~Hz}, \mathrm{H}=$ $\left.400 m, l_{x}=1 m, l_{y}=2 m, \sigma=0.05\right) \ldots \ldots \ldots$ 
5-13 Comparison of the backscattering strength estimated from the numerical simulated backscattering returns with the analytic solution. Sound speed variations have an exponential distribution. ( $f=400 \mathrm{~Hz}, \mathrm{H}=$

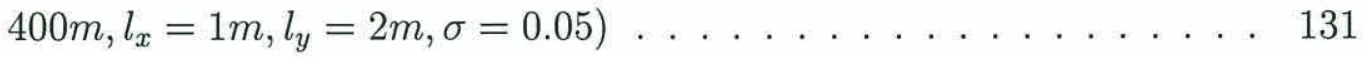

5-14 Backscattering from 2-D anisotropic sound speed variations with a power law type of distribution, averaging over 50 realizations $(f=$ $\left.400 H z, H=400 m, l_{x}=1 m, l_{y}=0.6 m, \sigma=0.05, \nu=1\right) \ldots .$.

5-15 Comparison of the backscattering strength estimated from the numerically simulated backscattered returns with the analytic solution. Sound speed variations have a power law distribution. $(f=400 \mathrm{~Hz}, \mathrm{H}=$ $\left.400 m, l_{x}=1 m, l_{y}=0.6 m, \sigma=0.05, \nu=1\right) \ldots \ldots$

5-16 Backscattering intensities for simulated backscattered returns due to sound speed variations with a Gaussian distribution, averaging over 50 realizations $(f=400 \mathrm{~Hz}, H=400 m, \sigma=0.05) \ldots \ldots . . .$.

5-17 The convergence test for backscattering from 3-D random sound speed variations with exponential distributions horizontally and vertically $\left(\sigma=0.05, l_{x}=l_{y}=2 m, l_{z}=0.5 m, f=400 H z, H=400 m, D=7.5 m\right)$

5-18 Comparison of the estimated backscattering strength from numerically simulated backscattered returns with the analytic solution. Sound speed variations are exponentially correlated horizontally and vertically. $\left(\sigma=0.05, l_{x}=l_{y}=2 m, l_{z}=0.5 m, f=400 H z, H=400 m, D=\right.$ $7.5 m) \ldots \ldots \ldots \ldots \ldots \ldots$

5-19 Backscattering intensities for simulated backscattered returns due to 2-D random sound speed variations only(dash-dotted line) and density variations only(dash line) and both(solid line). Sound speed variations have a power law distribution. $\left(\sigma=0.05, l_{x}=l_{y}=1 m, \beta=2, f=\right.$ $400 H z, H=400 m) \ldots \ldots \ldots \ldots$. . . . . . . . . . . . 137 
5-20 Backscattering strengths for simulated backscattered returns due to 2-D random sound speed variations only(dash-dotted line) and density variations only(dash line) and both(solid line). Sound speed variations have a power law distribution. $\left(\sigma=0.05, l_{x}=l_{y}=1 \mathrm{~m}, \beta=2, f=\right.$ $400 H z, H=400 m) \ldots \ldots \ldots \ldots \ldots \ldots \ldots \ldots$

6-1 Bathymetry and ship track in the ARSRP Site A sediment pond. . . 142

6-2 The experimental scenario. . . . . . . . . . . . . . . . . . . . 143

6-3 The DTAGS source and receiving array geometry. . . . . . . . . 144

6-4 (a) The received signal and (b) its spectrum at one hydrophone for a typical ping . . . . . . . . . . . . . . . . 145

6-5 The estimated receiving array configuration for a typical ping $\ldots . .146$

6-6 The beam pattern for a single-constraint beamformer: frequency $=450$ $\mathrm{Hz}$, look direction $=-45$ degrees. $\ldots \ldots \ldots \ldots \ldots \ldots$

6-7 The beam pattern for a five-constraints beamformer: frequency $=450$ $\mathrm{Hz}$, look direction $=-45$ degrees $\ldots \ldots \ldots \ldots \ldots$

6-8 Fluid bottom reverberation scenario. . . . . . . . . . . . . . 151

6-9 Schematic illustration of the scattering strength estimation. . . . . . 154

6-10 The estimated backscattering strength for the upper irregular region at the east side of the sediment pond. . . . . . . . . . . 156

6-11 The estimated backscattering strength for the lower irregular region at the east side of the sediment pond. . . . . . . . . . . . . . 157

6-12 The sensitivity of the estimated backscattering strength to the attenuation coefficient at $450 \mathrm{~Hz}$ for the upper irregular layer at the east side of the sediment pond. . . . . . . . . . . . . . . . . . . . . . 159

6-13 Model/data comparison for the upper irregular layer at the east side of the sediment pond. Sound speed fluctuations are described by a power law distribution with $l_{x}=l_{y}=20 m, l_{z}=0.7 m, \sigma=2.8 \%, \beta=3$, and $\nu=0.5$. The error bars show the standard deviation of the simulated backscattering strength. . . . . . . . . . . . . . . . 162 
6-14 Model/data comparison for the lower irregular layer at the east side of the sediment pond. Sound speed fluctuations are described by a power law distribution with $l_{x}=l_{y}=20 m, l_{z}=0.8 m, \sigma=2 \%, \beta=3$, and $\nu=0.5$. The error bars show the standard deviation of the simulated backscattering strength. . . . . . . . . . . . . . . . 163

6-15 Parameter studies for sound speed and density fluctuations described by a power law type of power spectrum: the effect of the aspect ratio. 165

6-16 Parameter studies for sound speed and density fluctuations described by a power law type of power spectrum: same aspect ratio but different correlation lengths, $\nu=0.5 \ldots \ldots \ldots \ldots \ldots \ldots$

B-1 Histogram of the simulated backscattering coefficients at $\theta=42$ de-

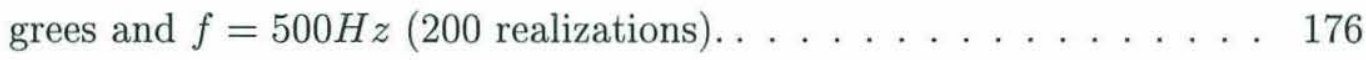

B-2 Histogram of the simulated backscattering coefficients averaged over 8 realizations at $\theta=42$ degrees and $f=500 \mathrm{~Hz}$ (200 realizations). . . 177

B-3 The estimated backscattering strength with standard deviations (error bars) over all the frequencies for the (a) upper and (b) lower irregular layers at the east side of the sediment pond. . . . . . . . 178 


\section{Chapter 1}

\section{Introduction}

\subsection{General Background}

Acoustic propagation in the ocean will inevitably encounter various types of inhomogeneities. Rough sea surfaces and seafloors, air bubbles, schools of fish, internal waves, and variations of sediment properties constitute only a small part of a wide spectrum. All of these inhomogeneities have the capability of reradiating a certain amount of incident acoustic energy, the process of which is called scattering.

With abilities to affect the distribution of the acoustic energy, scattering will undoubtedly play a significant role in sonar operation, which remains a primary means to explore the ocean. Analogous to optical scattering, which makes the world vivid to us, acoustic scattering enables us to "see" the underwater world. Broad applications include target detection and localization, geological surveys of the ocean seafloor, and monitoring of environmental changes, to name a few. On the other hand, contrary to its above role as signal, the reverberation can act as background noise to operations such as acoustic navigation and communication. Especially since it is induced by the transmitted signal itself, it would be extremely difficult to achieve a successful elimination without the knowledge of the excitation mechanisms. Needless to say, understanding scattering mechanisms is essential to sonar operations regardless of the role scattering is playing. 
Usually, acoustic scattering is categorized into rough surface scattering and volume scattering. For many years, scattering from rough surfaces has been the center of attention. Rough sea surfaces, exposed rocks on the seafloor, and large topographical features such as sea mountains and ridges are considered the dominant contributors to bottom scattering. On the other hand, bottom volume scattering has attracted more and more interest in recent years. Although less obvious and less well understood, more evidence shows that the inhomogeneities within the sediment can be a dominant factor in total bottom scattering, especially when the seafloor is relatively flat and/or the the bottom attenuation is small. In his early work on the measurement of the bottom backscattering strength, Merklinger [2] speculated that subbottom inhomogeneities may be primary contributors to the backscattered field. Jackson et al. $[3,4]$ and Lyons et al. [5] also recognized the importance of volumetric inhomogeneities in the bottom scattering. Tang et al.'s [6] analysis of high-frequency scattering data showed that the gas voids in the sediment are probable significant scatterers.

In order to understand a physical process such as scattering, we will have to resort to both experiment and modeling. Due to various reasons, sediment volume scattering is difficult to study. First of all, it is extremely difficult to directly measure the properties of the sediment. As a result, a lot of modeling work is based on very limited knowledge of the ground truth. Second, it is no easy task designing a good scattering experiment as well. In the usual deep-water scattering experiments, the source and receivers are close to the sea surface. Consequently, the insonified region is so large that the strong returns from sea mountains are likely to mask the weak returns from the sediment volume. In shallow water, the waveguide effects will inevitably complicate the scattered field, making it arduous to pinpoint the volume scattering effect $[7,8]$. Third, the conventional single source/single receiver configuration is unable to distinguish the volumetric scattered returns in different directions, which may result in misinterpretation of the data. To avoid all of the above problems, an ideal experimental scenario would be to have a deep-towed acoustic source and 
receiving array collecting data over a deep-sea sediment pond area, where the acoustic propagation is relatively simple in the absence of the waveguide effects manifested in shallow water and the scattered returns are primarily from the volume inhomogeneities in the sediment because the deep-sea water/sediment interfaces are normally flat. Fortunately for us, the experiment to be described next fit this scenario very well.

\subsection{Data Analysis Results of ARSRP Backscatter- ing Experiment}

As part of the Office of Naval Research Bottom/Subbottom Acoustic Reverberation Special Research Program (ARSRP), a backscattering experiment was conducted over a sediment pond on the western flank of the Mid-Atlantic Ridge in July 1993. A chirp source and vertical line array were deep-towed $200 \mathrm{~m}$ to $400 \mathrm{~m}$ above the seafloor, while the sediment thickness was up to $400 \mathrm{~m}$ in the middle of the sediment pond. For a transmitted acoustic source level of approximately $200 \mathrm{~dB}$ re $1 \mu \mathrm{Pa} @ 1 \mathrm{~m}$ and a source frequency of $250-650 \mathrm{~Hz}$, the acoustic signal can penetrate to the rock basement beneath the sediment layer in spite of sediment attenuation and geometrical spreading. Details of the experiment description are presented in Chapter 6. The following are the data processing results from Ref. $[9,10]$.

Taking advantage of the vertical line array, endfire beamforming yields signals which are dominated by normal incidence returns. With the ship moving across the sediment pond area, sediment profiling can be obtained by aligning the normal incidence returns with respect to reflection arrivals at the water/sediment interface. After employing an edge detection algorithm [11], the inferred sediment structure is shown in Fig. 1-1 at the east side of the sediment pond. Evidently, layering with gentle horizontal changes is the main characteristic of the sediment. However, some random features can be seen in two irregular layers beneath the water/sediment in- 


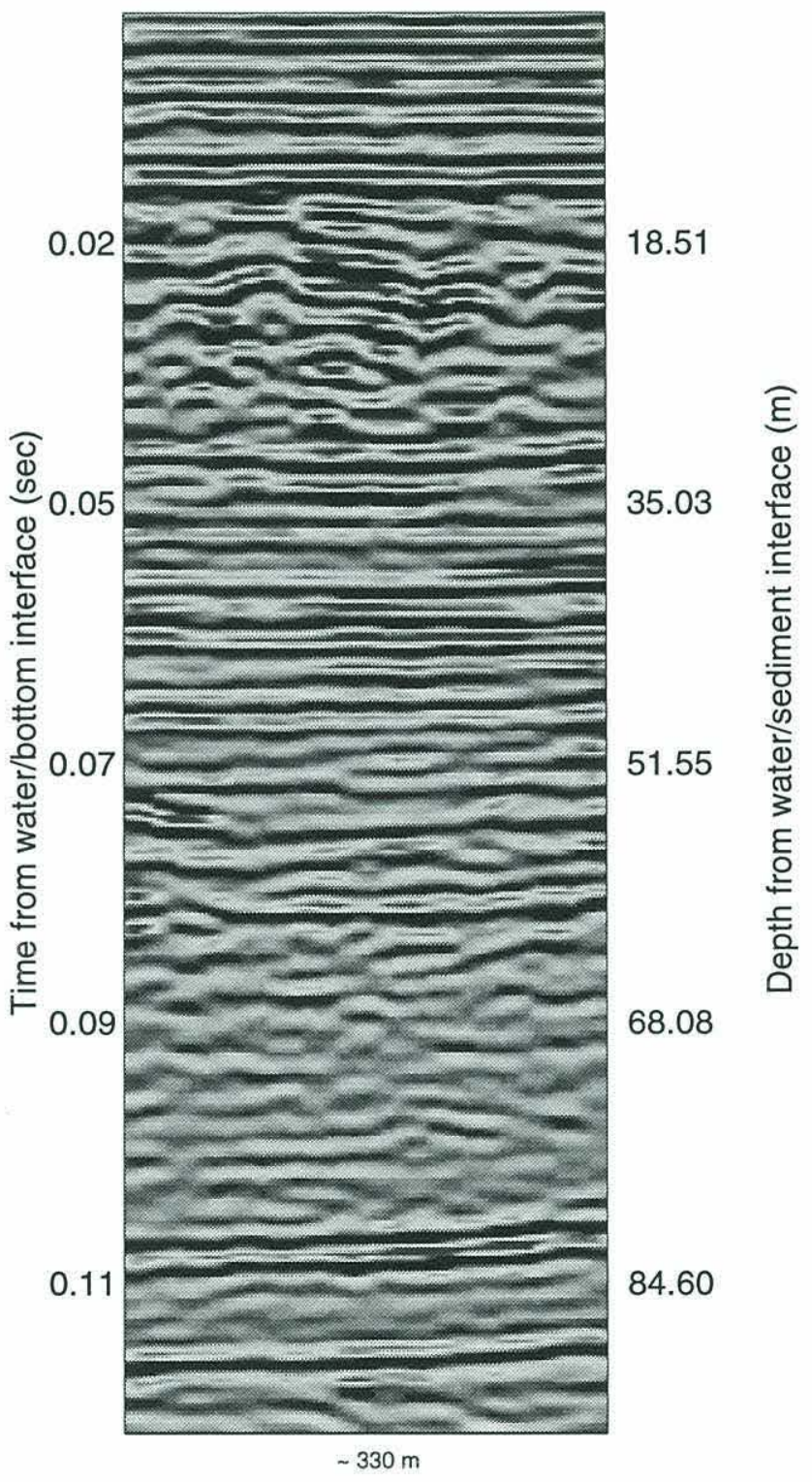

Figure 1-1: The inferred sediment structure at the east side of the sediment pond. 
terface, from $0.02 s$ to $0.045 s$ and from $0.065 s$ to $0.11 s$. A geological interpretation suggests that they are most likely to be turbidity layers [12]. What interests us is to find out whether the inhomogeneities contribute to backscattering in oblique directions. After subtracting the returns in normal and near normal incidence directions, the backscattered returns at oblique grazing angles can then be isolated by multiple constraints beamforming. We average the envelope of the beamformed results over 8 consecutive pings and further smooth the curve by a low-pass filter in order to detect any potential trend. The averaged envelope is shown in Fig. 1-2. Three groups of peaks appear in each look direction. Assuming that these peaks occur at horizontal interfaces and using a nominal sound speed of $1530 \mathrm{~m} / \mathrm{s}$, we have the best fit indicated by the solid lines. Here time 0 corresponds to the arrival time of the first water/sediment interface reflection. From the travel times, we find that the first two imaginary interfaces would reside at the locations of the above-mentioned two irregular layers, where actual interfaces don't appear to exist. We gather that the observed strong backscattering is probably caused by volumetric inhomogeneities. The third group is associated with sediment/basement interface according to travel time. Therefore, we tentatively conclude that the backscattered field in oblique directions is due to sediment inhomogeneities in the two irregular layers.

The same procedure is executed for pings collected at the west side of the sediment pond. Figure 1-3 shows the sediment structure. Interestingly, the upper irregular layer is absent on this side of the sediment pond. Therefore, if the above hypothesis that inhomogeneities in the irregular layers are the main contributors to the oblique backscattering is true, one would see only one group of peaks corresponding to the lower irregular layer instead of two groups of peaks at the beginning of the beamformed output record. Figure 1-4 shows the average envelope of oblique angle beamforming results. The striking point here is that the first group of peaks does disappear, which confirms our finding that the irregular layers beneath the water/sediment interface are the dominant contributors to backscattering in oblique directions. Notice that there is not a group of peaks close to time $0.02 s$ at 60 degree 


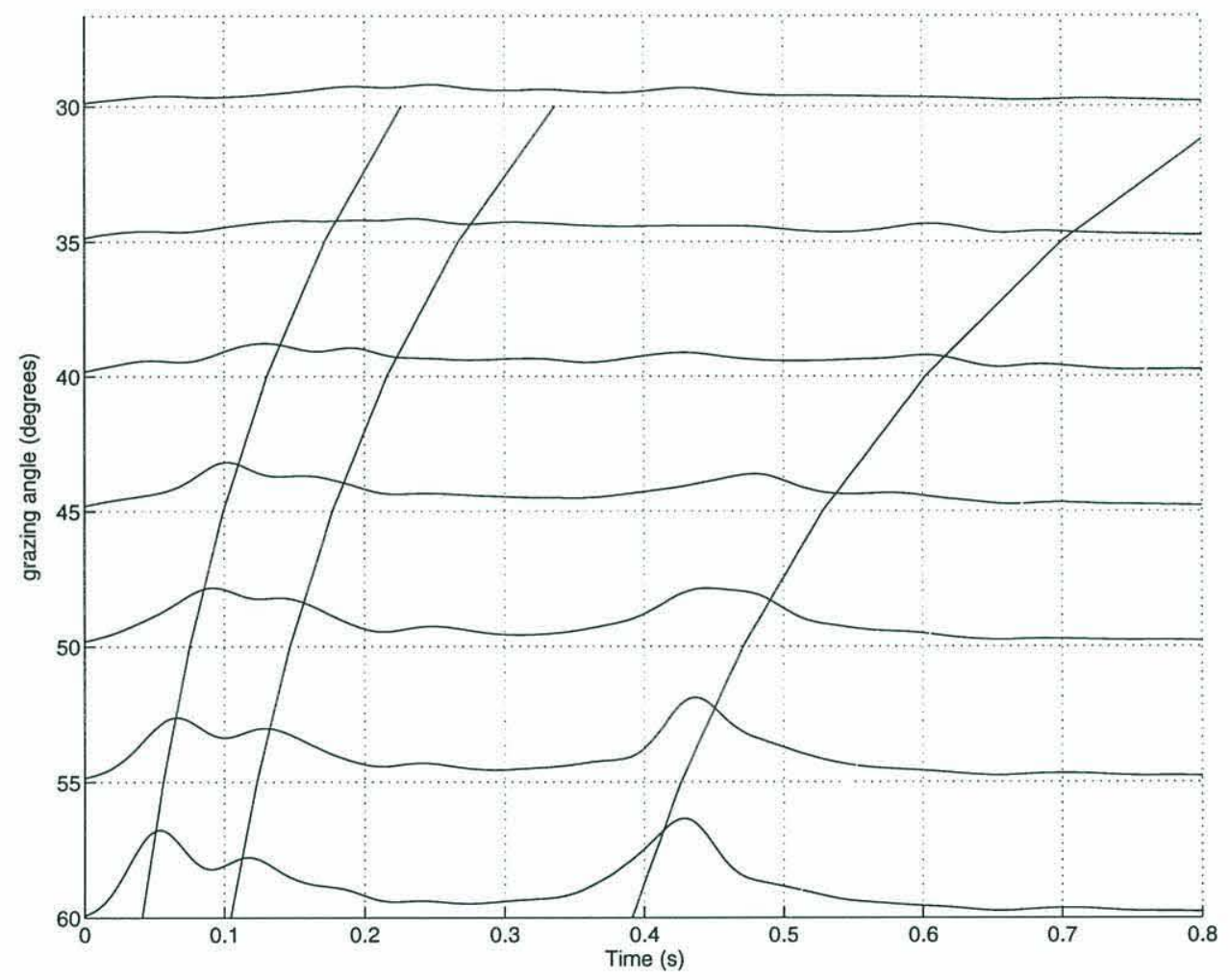

Figure 1-2: The average envelope of oblique angle beamforming results over 8 pings at the east side of the sediment pond (arbitrary units). 


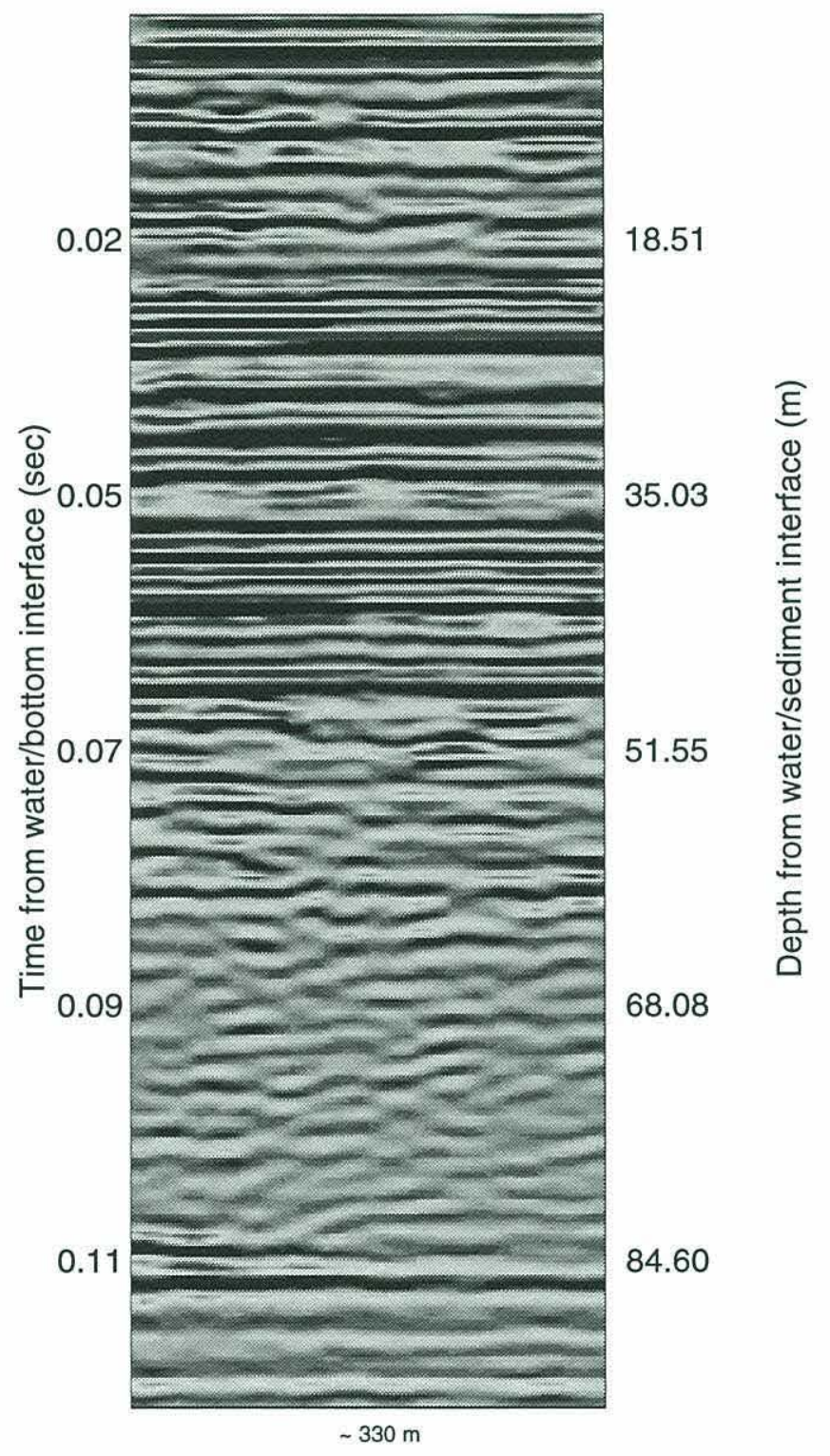

Figure 1-3: The inferred sediment structure at the west side of the sediment pond. 


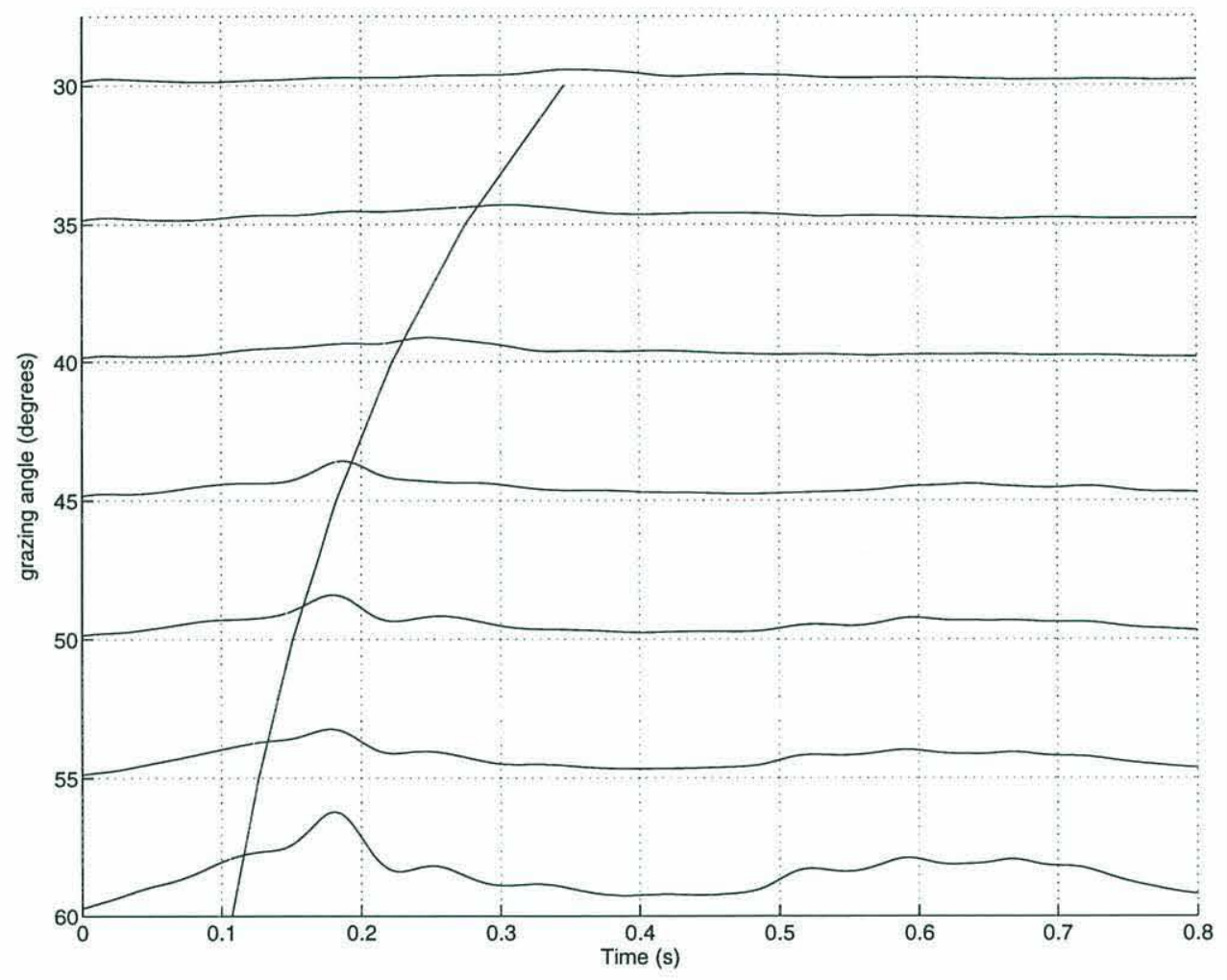

Figure 1-4: The average envelope of oblique angle beamforming results over 8 pings at the west side of the sediment pond (arbitrary units). 
grazing angle in both Fig. 1-2 and Fig. 1-4, which suggests the insignificance of backscattering at the water/sediment interface. This has been independently confirmed by Jeffrey and Ogden [13].

\subsection{Objective and Approach}

The results of experimental data analysis are encouraging. Now it is up to the modeling to help achieve better understanding of the scattering mechanisms. As a matter of fact, the objective of this thesis is to develop a monostatic backscattering model that can incorporate 3-D bottom volumetric inhomogeneities and to compare the results with the ARSRP backscattering data.

Since the propagation of sound between the source/receiver and the scatterer should be treated as an integral part of the scattering modeling, as the first step, efforts will be made to evaluate propagation approximations incorporated in Hines' [14], Ivakin's [15] and Mourad and Jackson's [1] scattering models. Comparisons with the full-wave solution obtained by a wavenumber integration method [16] are expected to show the validity and limitations of those approaches. The effectiveness of the equivalent surface scattering strength in characterizing the volume scattering process is going to be examined as well.

Due to the fact that the equivalent surface scattering strength is inappropriate in describing the angular dependence of the volume scattering when multi-path effects cannot be ignored, we want the model to possess an ability to overcome this. The most direct way to conduct a model/data comparison of bottom volume scattering is to compare them in the time domain. Scattering from volume inhomogeneities is usually considered as a random process, it is therefore practically impossible to synthesize time series which can match a single trace of the scattered field. Instead, one would try to compare the average shape, or frequency components, of the synthesized 
time series with that of the experimental data. A forward model that can incorporate random inhomogeneities and generate reverberation time series would definitely improve our capability of simulating scattering experiments.

In the model, the calculation of the incident field will be handled by a numerical wavenumber integration technique using OASES [16]. This would provide the model with versatility to deal with scattering involving complicated propagation scenario such as a sound speed gradient and even waveguide effects in shallow water. Similar to Morse and Ingard's [17] and Tang's [8] approach, a perturbation method will be applied to determine the scattered part of the field. Here, sound speed and den-

sity fluctuations in the fluid sediment are considered the cause of scattering. Rough surface scattering will not be included here but can be added in a straightforward manner. While the necessity to include poro-elastic effects in a sediment scattering model remains an open question [18], a fluid model is appropriate for the soft sediments and low frequencies under consideration. The volume inhomogeneities, i.e., the sound speed and density variations, are generated by the spectral method [19]. The random field is assumed to be correlated in three dimensions and defined by a power spectrum. By taking advantage of the fact that the source and receiver are both omnidirectional and co-located in monostatic backscattering experiment scenarios, the usually computationally intensive procedure can be carried out effectively. The model is expected to interpret the phenomena which have been observed in the ARSRP site A sediment scattering experiment $[9,10]$.

\subsection{Literature Review}

The modeling of scattering from both rough interfaces and volume inhomogeneities has been an active research area for many years, and the related literature is vast. We will present a brief overview of it. 
A large amount of work has been reported studying acoustic scattering from rough surfaces. There are two well-known theoretical methods for calculating acoustic scattering from rough surfaces: the Rayleigh-Rice method and the Kirchhoff method. Based on the small perturbation approximation, the Rayleigh-Rice method treats the rough surface as a perturbation to a smooth plane and the resulting scattering coefficient due to the presence of roughness is calculated. The Kirchhoff approximation approach is more pertinent to large-scale roughness, and the scattering surface is assumed to be sufficiently smooth so that the tangent plane at any point of the surface determines the reflection properties. Some efforts are also put into combining the above two methods in a composite model, treating small-scale roughness by the Rayleigh-Rice method and large-scale roughness by the Kirchhoff method [3]. A good review of theoretical work and experimental investigations on rough surface scattering can be found in Ogilvy's book [20].

It is generally accepted that backscattering from sediment volume inhomogeneities can contribute significantly to seafloor backscattering, especially at low frequencies, small grazing angles and where the bottom is flat. From the 1960's, a number of models have been developed in order to examine the underlying scattering mechanisms and to predict the strength of the backscattered signals.

Stockhausen [21] derived a volumetric backscattering strength expression assuming that the water-sediment interface is flat and refracting (with the consequent critical angle effect), and with the homogeneous sediment containing a uniform set of solid spherical particles which act to scatter the acoustic energy. Treating the small spheres as uncorrelated point scatterers, he employed Morse's expression [22] which is valid for scattering from spheres much smaller than a wavelength. In his model, Stockhausen represented all the scattering processes by a single volume backscattering cross section without further exploring any physical mechanisms.

Nolle et al. [23] at almost the same time developed their own model. They de- 
scribed the scattering from the sediment volume based on a random distribution of scattering amplitude per unit volume. The scattering strength distribution was considered to be centered at a mean value with random deviations. For simplicity, the scattering autocorrelation function was assumed to have the form of an exponential decay. The autocorrelation length was proportional to the particle size in the sediment volume, thus going one step further compared to Stockhausen's uncorrelated point scatterer model. Nolle et al. also conducted an experiment in the laboratory studying acoustic scattering from a smooth sand surface and compared their model with the collected data. It showed that the model had some difficulties in explaining the scattering from sub-critical angles.

Crowther [24] included both interface roughness and volume inhomogeneity effects in his ocean bottom backscattering model. Kuo's formula [25] for backscattering from the rough interface between two homogeneous fluids was used here. Extending Nolle et al.'s model, he assumed the correlation function for impedance fluctuations to be elliptic exponential, which was able to account for the anisotropy of the scatterers. However, this model also had problems with scattering at sub-critical angles when comparison with Nolle et al.'s laboratory backscattering data was made. Without knowledge of the detailed shape of the correlation function, it would be inappropriate to predict such characteristics as the frequency dependence of the scattering employing this model.

Morse and Ingard [17], studied volume scattering due to compressibility and density fluctuations in a free-space scenario. Their approach is still one of the best available methods in modeling volume scattering.

There were some singular features of the backscattering coefficient obtained in experimental measurements at sea [26] [27]. These included the frequency independence (or weak frequency dependence) over the frequency range 1-100 KHz and an angular dependence proportional to $\sin \theta$ for grazing angles $\theta$ from 5 to 50 degrees. 
In order to interpret this, Ivakin and Lysanov [28] proposed their statistical models on the basis of the geoacoustical model presented earlier by Lysanov [29]. The scattering was thought to come from "sharply anisotropic random inhomogeneities (fluctuations of the refractive index): large-scale in the horizontal plane and smallscale in depth" in the sediment. They used the Born approximation to derive an expression for the equivalent surface scattering coefficient, which was not dependent on the frequency if the absorption coefficient was proportional to frequency and the power spectrum of the inhomogeneities was inverse proportional to the wavenumber to the third order. Also, they extended the single-scale (horizontal correlation coefficient) model to two-scale and multi-scale models so as to account for the variability of the horizontal correlation coefficient in different regions. The interface effect was ignored by assuming that the changes in sound speed and density from the water to the water-saturated sediment were very small. The model prediction agreed very well with the data after the model/data fit of a free parameter: the ratio of the vertical and horizontal correlation lengths.

In their next paper [30], Ivakin and Lysanov revised their model to account for the interface roughness effect. The Kirchhoff approximation was applied to the rough interface backscattering case. The model studied in detail the influence of interface roughness on the volume backscattering cross section: for slow or nonreflecting bottoms, the volume scattering did not depend on whether the bottom interface was smooth or not; for fast bottoms, the roughness effect was significant in the subcritical grazing angle region. Physically, the reason for the latter result was because the rough interface would enhance the penetration of sound into the bottom medium at small grazing angles and therefore intensify the scattering of sound by volume inhomogeneities. They emphasized the nonadditiveness of the scattering effects due to volume inhomogeneities and water-bottom interface roughness, which was different from several other researchers who assumed that these two effects were uncorrelated. The authors did not include the possible lateral wave effects. 
Ivakin [15] extended his model for scattering from volume inhomogeneities again to deal with a stratified bottom. The correlation function for the random spatial fluctuations of the acoustical parameters was considered to be "statistically homogeneous in the horizontal plane and quasi-homogeneous with respect to the vertical coordinate". The model allowed considerable changes in the sound speed and the density with depth. He studied the linear increase in sound speed and density case and the linear decrease in sound speed case. The results showed good model/data agreement.

For the high-frequency $(10-100 \mathrm{kHz})$ bottom backscattering model proposed by Jackson et al. [3] in 1986, the composite roughness approximation was applied for the scattering due to interface roughness. To include the volume scattering contribution, they employed Stockhausen's formula [21] and accounted for the volume scattering by an equivalent surface backscattering coefficient. The assumption inherent in Jackson et al.'s approach was the neglect of any correlation between the scattering due to interface roughness and sediment inhomogeneities, which was different from Ivakin and Lysanov's model [30]. Multiple scattering was also ignored. The comparison of the model with two sets of data suggested that "in soft sediment, sediment volume scattering is likely to be more important than roughness scattering, except near normal incidence and for grazing angles smaller than the critical angle. For sand bottoms, roughness scattering is relatively more important". However, the volume scattering parameter was still obtained from model/data fits without any relationship to the sediment properties.

Mourad and Jackson [31] generalized Jackson et al.'s model [3] by including the sediment sound absorption in the interface boundary condition. They constructed some empirical relationships for estimating surficial values of the input geoacoustic parameters to the model using bulk measurements of logarithmic grain size $M_{z}$ proposed by Hamilton [32]. These parameters otherwise could only be obtained by direct measurement, which was not available for many datasets. Nevertheless, as they 
pointed out, the gradients of sound speed and density, which might play an important role in affecting the acoustical response of the sediment, were not included in this model.

Later, Mourad and Jackson [1] went one step further by including the gradient of sound speed in their low-frequency $(100-1000 \mathrm{~Hz})$ model. The volume scattering model was very similar to Ivakin's model [15] and they assumed "a distribution of uncorrelated omnidirectional point scatterers in the sediment causes the backscattering of sound". The model related the oscillation of the backscattering strength to the acoustic field within the sediment and suggested that the total backscattering from the ocean bottom was controlled by the processes affecting sediment volume scattering, for example strong layering. They also discussed the possible errors in the measurement of bottom scattering strength near the normal incidence direction using omnidirectional sources and receivers. For low-frequency backscattering, the questions for this model were how good the local plane-wave assumption inherent in the model was and the validity of attributing the volume scattering effect to an equivalent surface scattering process.

Hines [14] developed a backscattering model in an approach similar to Ivakin's [15]. He followed Chernov's work [33] and applied the Born approximation and the far-field assumption. Backscattering of the acoustic energy in his model was due to the sound speed and density fluctuation in the sediment, which he related to the fluctuation of porosity. The lateral wave effect was for the first time included in this model, attempting to interpret the phenomena observed in experiments at small grazing angles. An exponential decay correlation function was assumed and the model fit several published data very well. Yet a priori knowledge of the correlation function and variance of the porosity were needed for the model prediction. The approach of decomposing the incident spherical wave into a refracting plane wave and an evanescent wave was debatable. Later, he extended the model to deal with bistatic scattering $[34]$. 
Tang in his thesis [35] and Tang and Frisk in their several papers [36] [37] [38] discussed in detail the scattering from a random layer or half-space where the sound speed is assumed to be a constant plus a small random component. A self-consistent volume scattering model using a perturbation method was developed to model scattering from sound speed fluctuations. A coherent field reflection coefficient which included scattering loss could be calculated. An interesting point was that the spatial correlation length of the scattered field could be used to infer the correlation length of the scatterers. This provided a way of inverting for the bottom parameters critical to bottom scattering by measuring the scattered field using multiple receivers. Also taken into consideration was the anisotropy of the scatterers. Besides examining the combination of interface roughness and volume inhomogeneity effects, an attempt was made to solve the near-field problem in low-frequency scattering when the far-field assumption was not appropriate anymore.

Lyons et al. [5] extended Jackson et al.'s model [3] for scattering from the seafloor. In addition to the composite roughness model for interface scattering and Stockhausen's expression for volume scattering [21], they included the volume scattering from a random inhomogeneous continuum and scattering from subbottom interfaces. Their approach to calculating the scattering from the random inhomogeneous continuum was similar to Hines' work [14]. The compressibility and density variations were modeled. The correlation function could have different correlation lengths in the horizontal and in the vertical to allow for anisotropy in the volume scattering model. They employed the Born approximation to obtain the volume backscattering cross section, which was a free parameter in Jackson et al.'s model, before fitting it to Stockhausen's formula for an effective surface backscattering coefficient. They estimated all of the input parameters except the horizontal correlation length from core data. The comparison with data was very good. Because this model was more complicated and had more input parameters, its performance depended very much on the estimation of those parameters. 
Recently, Yamamoto [39] used the same approach as Chernov's [33] to model density and sound speed fluctuations in the sediment. The above fluctuations were described by a power law distribution and the parameters were estimated from crosswell tomography. The model compared with data favorably. However, the ray tracing type of propagation model would not be able to deal with scattering at low grazing angles for a fast bottom.

The modeling of volume scattering in a shallow water waveguide can be found in the work by Ellis [40], Tang [8] and Tracey [7].

For elastic media, in addition to compressional waves, shear waves and evanescent waves (e.g., Scholte waves at a fluid-elastic interface) may play important roles in bottom scattering. Their interactions generate some unique scattering features that cannot be observed in a fluid-fluid environment [41] [42] [43] [44]. For discrete elastic targets in a layered waveguide, the resultant scattering is treated by the transition matrix formalism [45] [46] or the boundary element method (BEM) [47]. However, the elasticity effect will not be considered in this thesis work.

On the experimental side, early investigations of bottom backscattering used an omnidirectional source and receiver. Mackenzie [48] presented the first deep-sea measurement results in 1961 . The source and receiver were close to the sea surface. He found that the bottom backscattering strength obeyed Lambert's law of diffuse reflection for grazing angles from 30 to 90 degrees, with the scattering constant (Mackenzie coefficient) having a value of $-27 \mathrm{~dB}$. This value didn't change for the frequencies 530 $\mathrm{Hz}$ and $1030 \mathrm{~Hz}$. In 1968, Merklinger [2] reported his experiment using a hydrophone and explosive charges suspended near the ocean bottom. The data analysis indicated that the reverberation due to the subbottom layer structure contributed significantly to the total bottom reverberation. This highlighted the importance of subbottom inhomogeneities in the prediction of the bottom backscattered field. 
Thanks to the evolution of technology, later experiments have used directional sources and/or receivers to obtain scattering data from the ocean bottom. Boehme et al. [49] conducted an experiment to study backscattering at low grazing angles in the frequency range $30-95 \mathrm{kHz}$, and found that the Lambert's law fit for grazing angles ranging from 2 to 10 degrees. Preston and Akal [50] employed a towed horizontal array and a suspended vertical array to measure ocean basin reverberation. They found that strong backscattering regions were related to basin topographic features. Hines and Barry [51] carried out an experiment in the Sohm Abyssal Plain with the source and receiver suspended close to the smooth seabed. Their acoustic array contained an omnidirectional hydrophone and a ring projector with vertical directivity. Different features were observed for backscattering from different bottom types. Jackson and Briggs [4] used a towed platform equipped with planar arrays to study high frequency bottom backscattering in three different regions. Sediment volume scattering was found dominant in two of the three sites.

\subsection{Contributions}

This thesis has advanced the state-of-the-art in several ways. These include:

- Evaluation of sound propagation models used in bottom volume scattering studies. The validity and limitations of some widely used models have been recognized and the equivalent surface scattering strength is found insufficient to characterize the mid- and low-frequency volume scattering process when multi-path effects are severe.

- Implementation of a numerical monostatic backscattering model with the ability to incorporate 3-D volume inhomogeneities and to compare with experimental data effectively. Density fluctuations have been confirmed to be the main contributor to backscattering, while the anisotropy of the statistical distribution of 
the inhomogeneities in both horizontal and vertical directions is found to have a profound influence on the angular dependence of the backscattering strength. In addition, the synthesized time series can help to design an experiment and provide predictions of reverberation fields.

- Formulation of the spectral method for the generation of azimuthally integrated 3-D random fields. This approach provides the opportunity to understand the full 3-D phenomena with a manageable computational load.

- Comparisons with the ARSRP backscattering data. The good fit between the model and data has proved the versatility of the model. The power law type of power spectrum is found to be the best description of sound speed and density fluctuations in the sediment that gives rise to excellent model/data comparisons.

\subsection{Overview of the Thesis}

Chapter 2 begins by reviewing the definition of the scattering cross section and the propagation models by Hines, Ivakin and Mourad and Jackson. Comparisons with the exact solution obtained by OASES are then carried out for three different type of fluid bottom models. The pros and cons of each propagation model are discussed. Meanwhile, the validity of the equivalent surface scattering strength in characterizing volume scattering is also evaluated.

In Chapter 3, the formulations for scattering due to sound speed and density fluctuations are derived. In addition, the power spectra and correlation functions that are going to be investigated later are presented. Analytic solutions for scattering in a free-space scenario are used to study the effects on backscattering imposed by different distributions and different correlation lengths of sound speed variations.

Chapter 4 presents a scheme to generate 3-D azimuthally-summed random fields by the spectral method. Numerical examples are also given for verification of this 
approach.

Chapter 5 describes the procedures for generating backscattered time series. Then the focus shifts to the verification of the numerical model. The effect of density fluctuations is studied at the end.

In Chapter 6, we present a detailed description of the ARSRP backscattering experiment. A brief introduction of the data processing methodology is then given. The estimation of backscattering strength from data and the selection of model parameters are then discussed. Data from the ARSRP experiment are compared to numerical simulations.

Chapter 7 summarizes the physical insights gained from the modeling and suggests directions for future work. 


\section{Chapter 2}

\section{Evaluation of Sound Propagation}

Models Used in Bottom Volume

\section{Scattering Studies}

The proper evaluation of sound propagation between sources/receivers and scatterers is important in characterizing bottom volume scattering. In this chapter, several sound propagation models used in bottom volume scattering studies are evaluated and their results compared to the exact solution obtained through a numerical wavenumber integration technique. It is found that Hines' approach [14] works well for the two isovelocity half-space case except when the grazing angle is close to the critical angle. The far-field approximation, given by Ivakin [15] and Mourad and Jackson [1], has a performance depending upon the sound speed structure in the sediment. For an isovelocity slow bottom, it agrees well with the exact solution. However, discrepancies arise for an isovelocity fast bottom or a bottom with a complex sound speed structure. In addition, the appropriateness of using the equivalent surface scattering strength as a function of grazing angle in volume scattering characterizations is studied. In conclusion, precautions need to be taken in modeling both the propagation effects and the scattering mechanisms associated with the bottom volume scattering process. 
This chapter is structured as follows: In the first section, we discuss the classical scattering problem and the definition of the conventional scattering cross section. The concept of an equivalent surface scattering strength which incorporates bottom volume scattering is also reviewed; for the second section, we describe the propagation models, and in the next section, we compare the results of these models for some typical cases of interest.

\subsection{Statement of the Problem}

Acoustic wave scattering from the ocean bottom generally includes rough water/bottom interface scattering and subbottom volume scattering. While rough surface scattering has been the focus for many years, recent evidence shows that volume scattering, due to inhomogeneities and/or scattering layers within the sediment, could contribute significantly to bottom reverberation.

The conventional quantities used to characterize the above two processes are the surface scattering coefficient and the volume scattering coefficient [52], which historically are defined in the context of a plane wave being scattered by scatterers confined in an otherwise homogeneous medium. In ocean bottom scattering, however, the incident field is normally not a plane wave. The multipath and refractive effects resulting from bottom sound speed structure complicate the scattering process and make it extremely difficult to pinpoint the scattering element in the classical sense. This problem will not diminish when the bottom volume scattering process is treated by an equivalent surface scattering process with the parameter of equivalent surface scattering coefficient being used. Therefore, the credibility of the scattering strength estimated from experimental data by this means needs to be examined.

Bottom volume scattering modeling usually include two components: the scattering part, which encompasses the appropriate scattering mechanisms and the propa- 
gation part, which takes into account propagation from the source to the scatterer and from the scatterer to the receiver. We will concentrate on propagation models in this study, which is equivalent to evaluating the Green's function between the source/receiver and the scatterer. The necessity to include poro-elastic effects in the scattering model remains an open question [18], and we will regard the bottom as an acoustic fluid. The free-space Green's function, together with the transmission coefficient at the water/bottom interface has been used in some work [24, 53]. Despite its simplicity, it cannot incorporate the interface wave contribution at subcritical grazing angle for a fast bottom. Since we are interested only in the methods that are good for the entire angular regime, this method will not be discussed in this paper. Ivakin [15] and Mourad and Jackson [1] considered the incident wave on the interface to be a plane wave, with the incident direction varying over the insonified region to match the true incident direction. For convenience, we call it the far-field approximation in this paper. This approach is actually a simplified version of the stationary phase method with only the most dominant stationary point contribution being included. Meanwhile, Hines [14] followed the stationary phase approach of Westwood [54] to find the transmitted field due to a point source in the two isovelocity half-space case.

The goal of this chapter is to demonstrate the validity and limitations of the above-mentioned propagation models based on realistic geoacoustic parameters. Results from these models are compared with the exact solution obtained through a numerical wavenumber integration technique. Their influence on the estimation of the equivalent surface scattering strength is also studied in this chapter.

\subsection{The Scattering Cross Section}

The conventional scattering cross section is defined when describing the scattering phenomenon associated with a single scatterer (e.g., a particle), as shown in Fig. 2-1. For simplicity, the incident plane wave is assumed to be propagating in the positive 


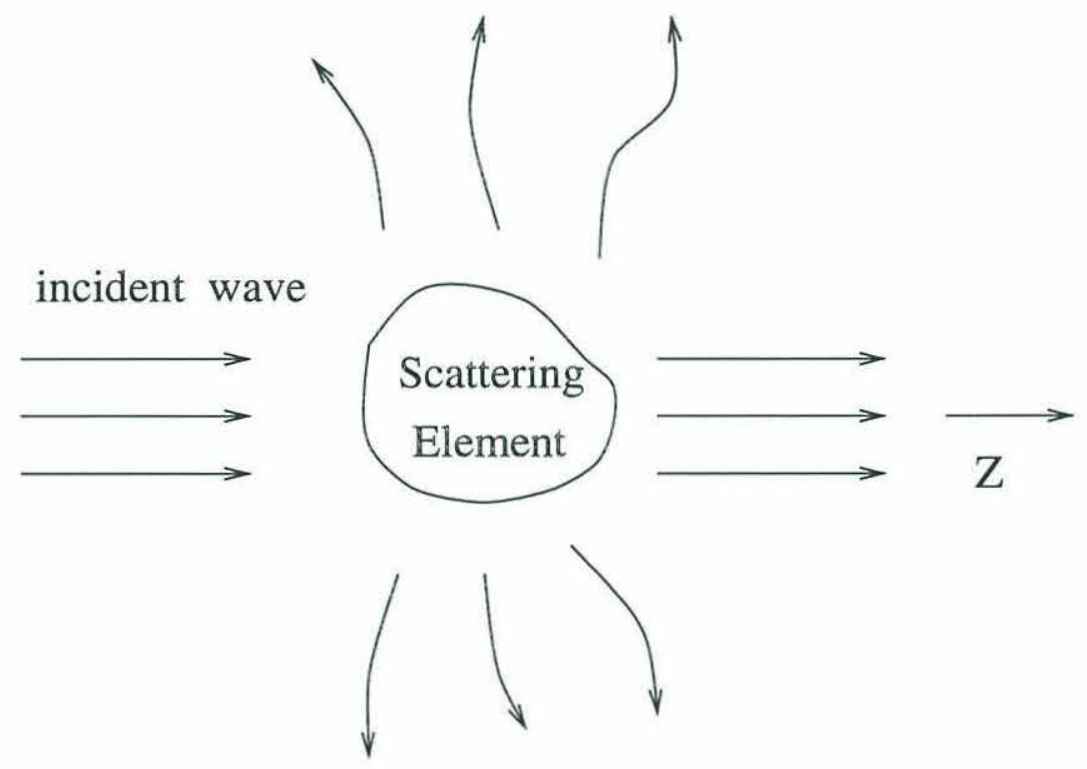

Figure 2-1: Geometry of the classical scattering problem.

$z$ direction with unit amplitude and suppressed time dependence $e^{-i \omega t}$ :

$$
\psi_{i}=e^{i k z} .
$$

The time dependence is chosen in the same convention as that in Ivakin's and Hines' work. However, bear in mind that I will change it to $e^{i \omega t}$ starting from Chapter 3 in order to be consistent with the convention used in statistic theory and random field generation. The scattered wave behaves as a spherical wave in the far field and is measured at infinity:

$$
\psi_{s}=f(\theta, \phi) \frac{e^{i k r}}{r}, \text { for } r \rightarrow \infty,
$$

where $r, \theta$ and $\phi$ are spherical coordinates with the origin at the scatterer. The scattering cross section is then defined as [55]

$$
\sigma(\theta, \phi)=|f(\theta, \phi)|^{2} .
$$

It is a measure of the scattered power in the $(\theta, \phi)$ direction per unit solid angle, per unit incident intensity. Because the incident field is a plane wave and the observa- 


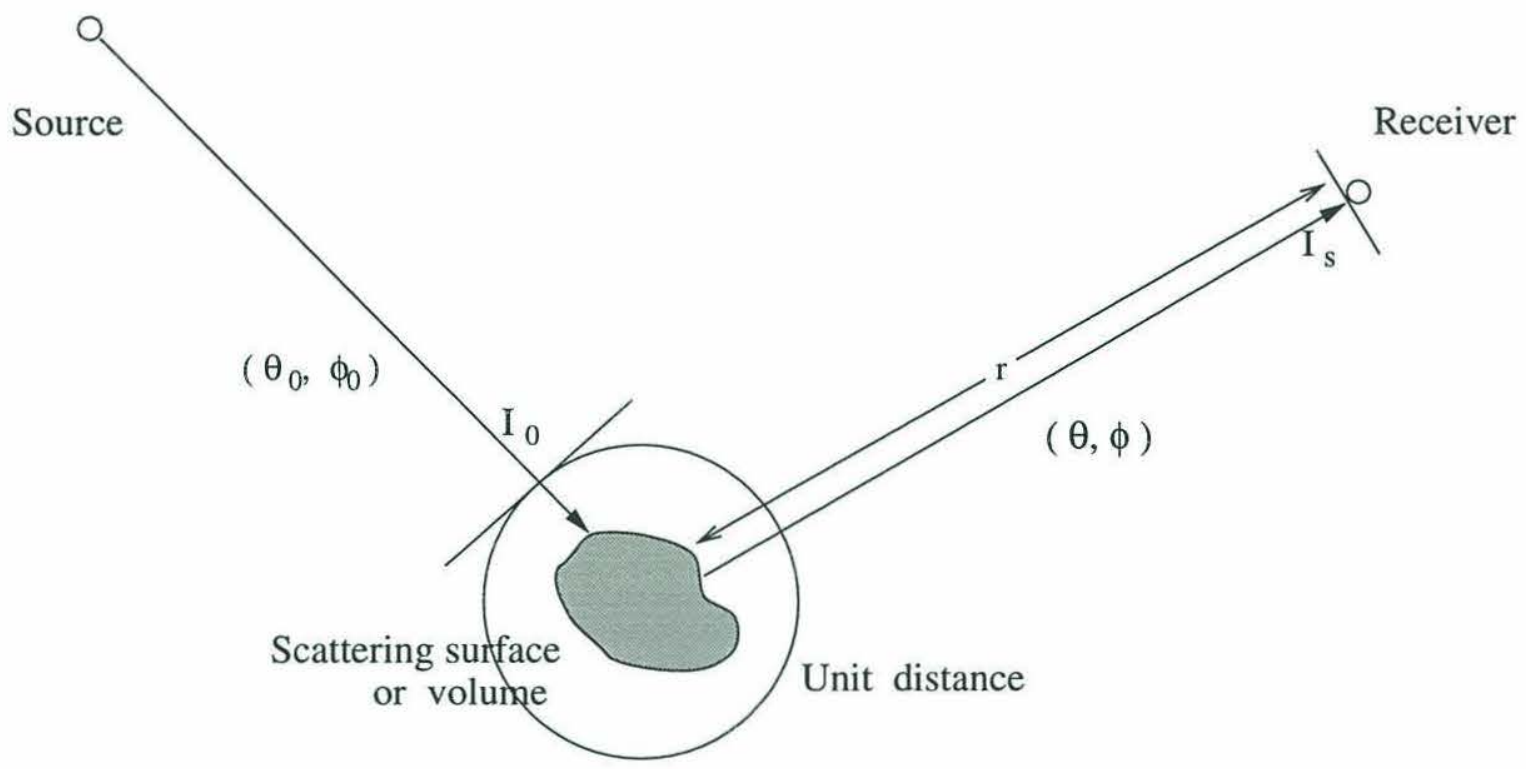

Figure 2-2: Schematic illustration of the surface and volume scattering coefficient definitions.

tion point is at infinity, $\sigma$ is a characterization of the scatterer independent of the source/receiver geometry [37].

As stated before, in bottom scattering studies, scattering by the rough water/bottom interface and volume inhomogeneities within the bottom are two key processes. Any type of scattering modeling will inevitably encounter the problem of describing the scattering ability of a surface area and/or a volume. Figure 2-2 shows the concept underlying the definitions of a surface scattering coefficient $\sigma_{s}$ and a volume scattering coefficient $\sigma_{v}$, which is similar to $\sigma$. Plane wave incidence is again assumed here, with the source and receiver in the far field. The incident intensity $I_{0}$ is measured at unit distance away from the scatterer. The scattered wave intensity $I_{s}$ is obtained at the receiver and then multiplied by $r^{2}$ in oder to compensate for spherical spreading of the scattered wave. Similar to the definition of $\sigma$, the scattering ability is characterized by the ratio of the scattered wave intensity to the incident intensity at unit distance from the scatterer per unit area or volume. It can be expressed in 
the following formula [52]:

$$
\sigma_{s}=I_{s}(\theta, \phi) r^{2} /\left[I_{0}\left(\theta_{0}, \phi_{0}\right) d S\right],
$$

and

$$
\sigma_{v}=I_{s}(\theta, \phi) r^{2} /\left[I_{0}\left(\theta_{0}, \phi_{0}\right) d V\right],
$$

where $\left(\theta_{0}, \phi_{0}\right)$ is the incident direction, $(\theta, \phi)$ is the scattering direction, and $d S$ and $d V$ are the insonified area and volume, respectively.

Several things need to be noticed about the introduction of $\sigma_{s}$ and $\sigma_{v}$. First, instead of a single scatterer, there exists a distribution of scatterers in the area $S$ or volume $V$. Therefore, $\sigma_{s}$ and $\sigma_{v}$ represent a measurement of the average scattering ability of the insonified surface or volume. Second, in the definition of $\sigma$, a plane wave incident on the scatterer is assumed in order to exclude the effect of source position. Similarly for $\sigma_{s}$ and $\sigma_{v}$, the incident wavefront is considered planar or locally planar, with the source in the far field. However, due to the possible multipath and refractive effects, this is generally not true for bottom scattering problem. Third, for the same reasons, the scattered wave is not simply a spherical wave. As a result, the spreading loss at a distance $r$ cannot be compensated for by the factor $r^{2}$, and in general, the phase is incorrectly calculated as well.

Despite the increasing interest in bottom volume scattering, it remains a difficult issue to characterize the scattering process. Unlike the rough surface scatterers which are concentrated at the water/bottom interface, the scatterers associated with volumetric scattering may be distributed over a large region. Scatterers may be located immediately beneath the water/bottom interface or a certain distance below the seafloor. As illustrated in Fig. 2-5, if we have a fast bottom and the scatterer is not at the interface but with $\beta$ smaller than critical grazing angle, the scattered wave can reach the receiver through two paths, therefore at two different angles. The path lengths are different so that the arrival times are not the same. As will be discussed 


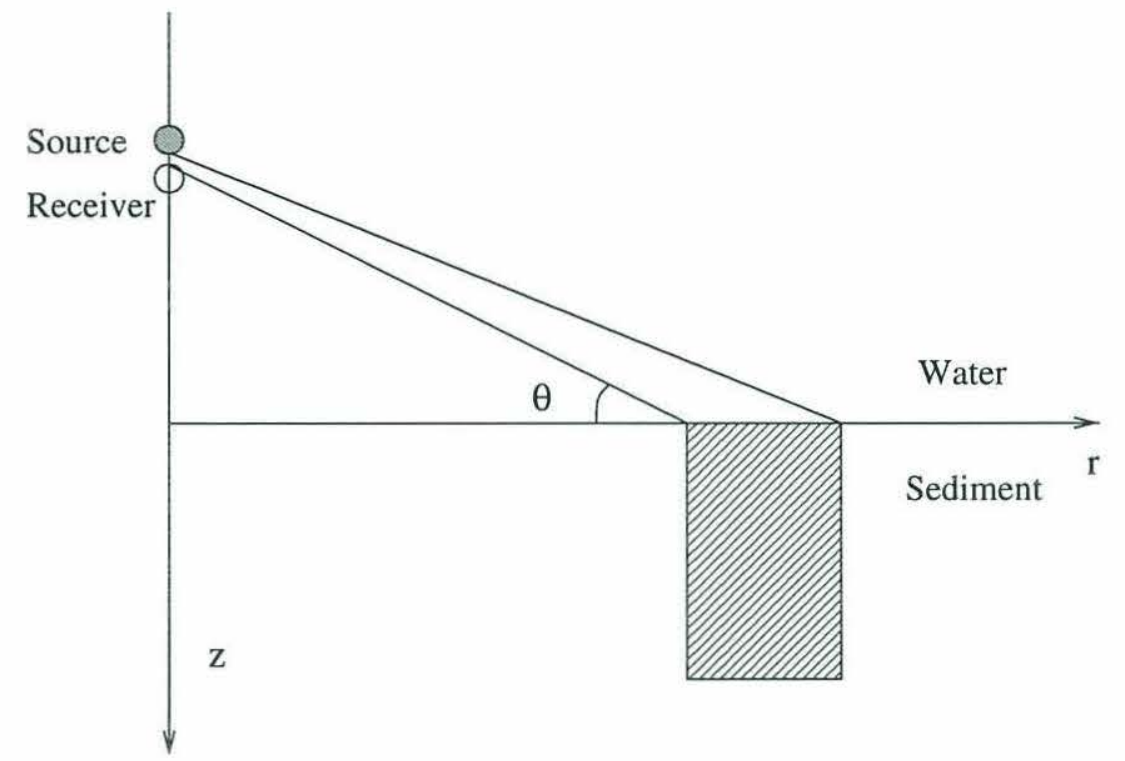

Figure 2-3: Schematic illustration of the equivalent surface scattering coefficient definition.

later, the above multi-path effect will pose nontrivial problems for scattering strength estimations.

When the bottom attenuation is sufficiently high and the depth of acoustic penetration is much smaller than the distance between the source and the sediment, scattering from volume inhomogeneities within the bottom can be described by an equivalent surface scattering process [1]. As shown in Fig. 2-3, scattering from the shaded region, which is a slice of the scattering volume in an axisymmetric coordinate system, is attributed to scattering from the surface area above it. All of the scattering are considered all from the grazing angle $\theta$ because the difference would be minimal when the observation point (i.e., receiver) is very far away. The equivalent surface scattering coefficient $\sigma_{e s}$, instead of the volume scattering coefficient $\sigma_{v}$, is then defined to quantify the scattering process. Mourad and Jackson [1] present a thorough description of this approach, with the result

$$
\sigma_{e s}=\int \sigma_{v}\left|\Psi_{1}\right|^{4} d z,
$$


where

$$
\Psi_{1}=\Psi_{v} / \Psi_{s} .
$$

Here $z$ is the depth coordinate, $\Psi_{v}$ and $\Psi_{s}$ are the incident field as it would be measured at the volume element $d V$ and the area element $d A$, respectively. $\sigma_{e s}$ can be viewed as a measure of the scattering contribution from the whole shaded region. $\Psi_{1}$ is the normalized field as if the scatterers were at the surface.

The concept of an equivalent surface scattering coefficient has been used extensively in scattering data analysis. In the high-frequency, high-attenuation case, the incident wave may penetrate to relatively shallow depths. However, at low frequencies and attenuations, the ensonification can encompass a substantial portion of the sediment column. The sediment layering and the sound speed gradient cause the scatterers to be ensonified by an incident field consisting of refracted, multiple reflected paths. Even if we neglect the multiple scattering effect, which is to ignore the rescattering of the scattered wave, it is still very difficult to relate the scattered returns recorded in an experimental time series to the corresponding scatterers. As can be seen in Fig. 2-3, the thickness of the shaded region would be very large for deep penetration. In the above calculation of the equivalent surface scattering strength, all the contributions from each shaded "vertical bar" would be ascribed to only one scattering angle, which can be justified for shallow penetration in a high-frequency situation but is invalid in the low-frequency case. In fact, the volume element could affect the scattering at different angles due to multipath effects. As a result, the angular dependence of the predicted scattering strength might be error-prone. In a shallow water environment, the limited water depth would further deteriorate the problem, since the distance between the source/receiver and the water/bottom interface is confined. All of the above would directly affect the effectiveness of using the scattering strength as a criteria in a model/data comparison. 


\subsection{Bottom Propagation Models}

Propagation, i.e., the Green's function, plays an important role in the modeling of the scattering process. In this section, three methods will be described for calculating the propagation from a point source in the water column to a scatterer in the sediment. The propagation from the scatterer to a receiver in the water can be obtained accordingly by the principle of reciprocity.

The wave equation in an inhomogeneous medium [56] can be written as

$$
\rho(\mathbf{r}) \nabla \cdot\left(\frac{1}{\rho(\mathbf{r})} \nabla p(\mathbf{r}, t)\right)-\frac{1}{c^{2}(\mathbf{r})} \frac{\partial^{2} p(\mathbf{r}, t)}{\partial t^{2}}=f(\mathbf{r}, t),
$$

where $p(\mathbf{r}, t)$ represents the pressure, $\rho(\mathbf{r})$ represents the density, $c(\mathbf{r})$ represents the sound speed, and $f(\mathbf{r}, t)$ represents the source term as a function of space $\mathbf{r}$ and time $t$. If constant density and harmonic time dependence $[\exp (-i \omega t)]$ are assumed, Eq.2.8 becomes the Helmholtz equation

$$
\left[\nabla^{2}+k_{0}^{2}(\mathbf{r})(1+\epsilon(\mathbf{r}))\right] P(\mathbf{r}, \omega)=F(\mathbf{r}, \omega)
$$

where $k_{0}(\mathbf{r})$ is the medium wavenumber for the background sound speed $c_{0}(\mathbf{r})$ and frequency $\omega$, with

$$
k(\mathbf{r})=\frac{\omega}{c_{0}(\mathbf{r})},
$$

and

$$
\begin{aligned}
f(\mathbf{r}, t) & =F(\mathbf{r}, \omega) e^{-i \omega t} \\
p(\mathbf{r}, t) & =P(\mathbf{r}, \omega) e^{-i \omega t} .
\end{aligned}
$$

Parameter $\epsilon(\mathbf{r})$ is the perturbation to the wavenumber due to the inhomogeneous sound speed. Using a perturbation method, the total field $P(\mathbf{r})$ (for simplicity, $\omega$ will be implicit from now on) can be expressed as the summation of the incident field 
$P_{0}(\mathbf{r})$ and the scattered field $P_{s}(\mathbf{r})$ :

$$
P(\mathbf{r})=P_{0}(\mathbf{r})+P_{s}(\mathbf{r}),
$$

where the incident field satisfies

$$
\left[\nabla^{2}+k_{0}^{2}(\mathbf{r})\right] P_{0}(\mathbf{r})=F(\mathbf{r}) .
$$

We assume a point source at $\mathbf{r}=\mathbf{r}_{\mathrm{s}}$ and the Green's function can be obtained from

$$
\left[\nabla^{2}+k_{0}^{2}(\mathbf{r})\right] G\left(\mathbf{r}, \mathbf{r}_{\mathbf{s}}\right)=\delta\left(\mathbf{r}-\mathbf{r}_{\mathbf{s}}\right),
$$

and the boundary conditions. The scattered field $P_{s}$ satisfies

$$
\left[\nabla^{2}+k_{0}^{2}(\mathbf{r})\right] P_{s}(\mathbf{r})=-k_{0}^{2}(\mathbf{r}) \epsilon(\mathbf{r}) P(\mathbf{r}) .
$$

The integral equation for the solution of the scattered field is

$$
P_{s}(\mathbf{r})=-k_{0}^{2}(\mathbf{r}) \int G\left(\mathbf{r}, \mathbf{r}^{\prime}\right) \epsilon\left(\mathbf{r}^{\prime}\right) P\left(\mathbf{r}^{\prime}\right) d \mathbf{r}^{\prime} .
$$

From Eq.2.15, it can be seen that the scattered field and the Green's function are closely related. The Green's function, which characterizes the propagation from the source to the scatterer and from the scatterer to the receiver, plays an important role in modeling of the bottom scattering process. In this section, three methods will be described for calculating the propagation from a point source in the water column to a scatterer in the sediment. The propagation from the scatterer to a receiver in the water can be obtained accordingly by the principle of reciprocity.

There are several ways to solve Eq.2.13. For simplicity, we will consider a rangeindependent, horizontally stratified environment, which If we choose a cylindrical coordinate system, with the vertical $z$-axis passing through the source and the r-axis being parallel to the interfaces, it becomes an axisymmetric propagation problem. At 


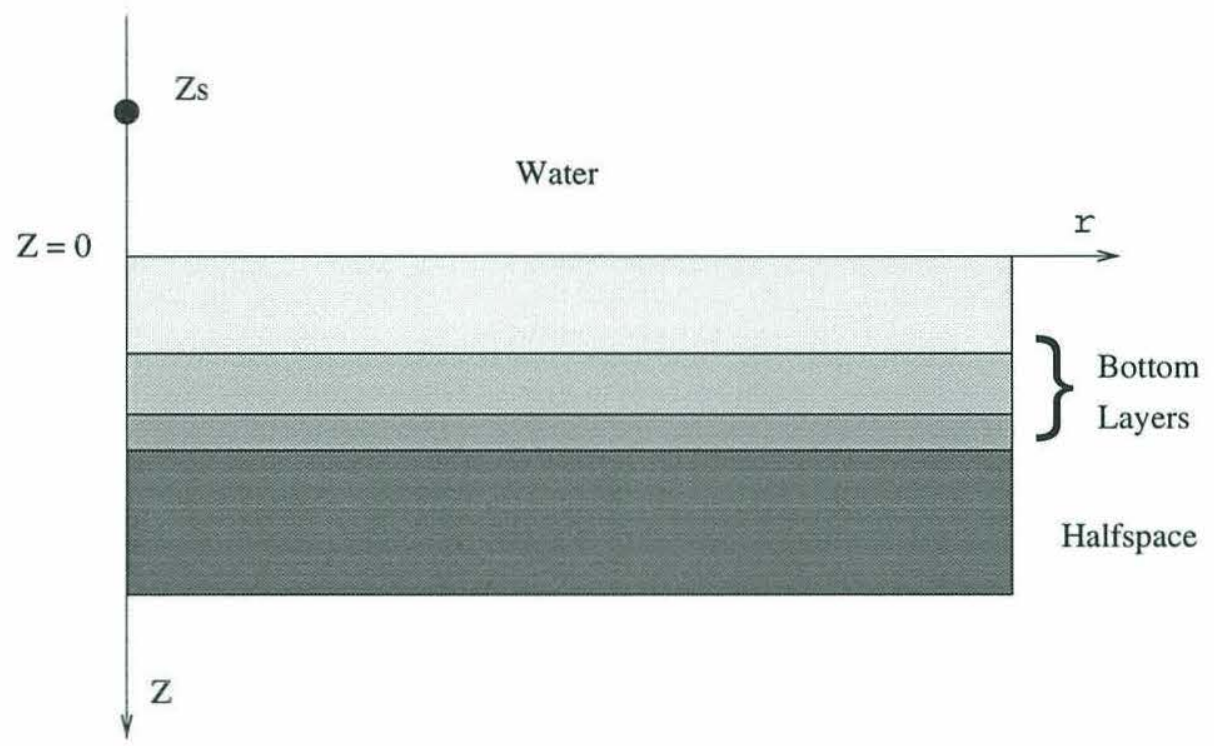

Figure 2-4: The experimental scenario.

this point, we will assume unit source strength. The Green's function can then be calculated by means of wavenumber integration. For the scenario shown in Fig. 2-4, we have the Green's function in the sediment $G(r, z)$ as follows,

$$
\begin{aligned}
G(r, z) & =\int_{0}^{\infty} g\left(k_{r}, z\right) k_{r} J_{0}\left(k_{r} r\right) d k_{r} \\
& =\frac{1}{2} \int_{-\infty}^{\infty} g\left(k_{r}, z\right) k_{r} H_{0}^{(1)}\left(k_{r} r\right) d k_{r}
\end{aligned}
$$

where $k_{r}$ is the horizontal wavenumber, $J_{0}$ is the zeroth order Bessel function, $H_{(0)}^{1}$ is the zeroth order Hankel function of the first kind and $g\left(k_{r}, z\right)$ satisfies the equation

$$
\left[\frac{d^{2}}{d z^{2}}+\left(k^{2}(z)-k_{r}^{2}\right)\right] g\left(k_{r}, z\right)=0
$$

and the boundary conditions of continuity of normal particle velocity and pressure across each interface. When $k_{r} r \gg 1$, we have

$$
G(r, z)=\frac{1}{\sqrt{2 \pi r}} \int_{-\infty}^{\infty} g\left(k_{r}, z\right) \sqrt{k_{r}} e^{i\left(k_{r} r-\frac{\pi}{4}\right)} d k_{r}
$$

Eq.2.18 can be evaluated numerically. The procedure of implementation can be 
found in Schmidt's SAFARI manual [16] and Ref.[57]. In fact, the numerical solution of the wave equation in Section III is obtained primarily by using SAFARI. One of the advantages of SAFARI is that it generally provides stable results for complicated sediment structures.

A point source is located at $\left(0, z_{s}\right)$. If an isovelocity water column is assumed so that the water wavenumber $k_{0}=\omega / c_{0}$, the depth-dependent Green's function in the water column will be

$$
g_{w}\left(k_{r}, z\right)=-\frac{e^{i k_{z_{0}}\left|z-z_{s}\right|}}{4 \pi i k_{z_{0}}}
$$

where $k_{z_{0}}=\sqrt{k_{0}^{2}-k_{r}^{2}}$ is the vertical wavenumber in the water. By matching the boundary conditions at the water/sediment interface $z=0$, it is clear that the depth-dependent Green's function in the sediment can be expressed in terms of $-e^{i k_{z_{0}}\left|z_{s}\right|} / 4 \pi i k_{z_{0}}$. In other words, we have

$$
g\left(k_{r}, z\right)=-g_{1}\left(k_{r}, z\right) \frac{e^{i k_{z_{0}}\left|z_{s}\right|}}{4 \pi i k_{z_{0}}}
$$

where $g_{1}\left(k_{r}, z\right)$ can be viewed as the solution to Eq.2.17 with a plane wave incident on the water/sediment interface. Using the coordinates shown in Fig. 2-4, Eq.2.18 now becomes

$$
G(r, z)=\frac{e^{i \frac{\pi}{4}}}{\sqrt{2 \pi r}} \int_{-\infty}^{\infty} \frac{g_{1}\left(k_{r}, z\right) \sqrt{k_{r}} e^{i\left(k_{r} r-k_{z_{0}} z_{s}\right)}}{4 \pi k_{z_{0}}} d k_{r} .
$$

The stationary phase method is often used to evaluate an integral of this type. Following Ivakin's approach [15], one obtains

$$
G(r, z)=\frac{e^{i k_{0} R}}{4 \pi R} g_{1}\left(k_{r}, z\right),
$$

where $R$ is the distance between the source and the point at the interface right above the scatterer in the sediment, as shown in Fig. 2-5. For simplicity, a two half-space scenario is being considered. In the above approach, the propagation can be interpreted as a spherical wave traveling from the source to the interface point $B$ above 


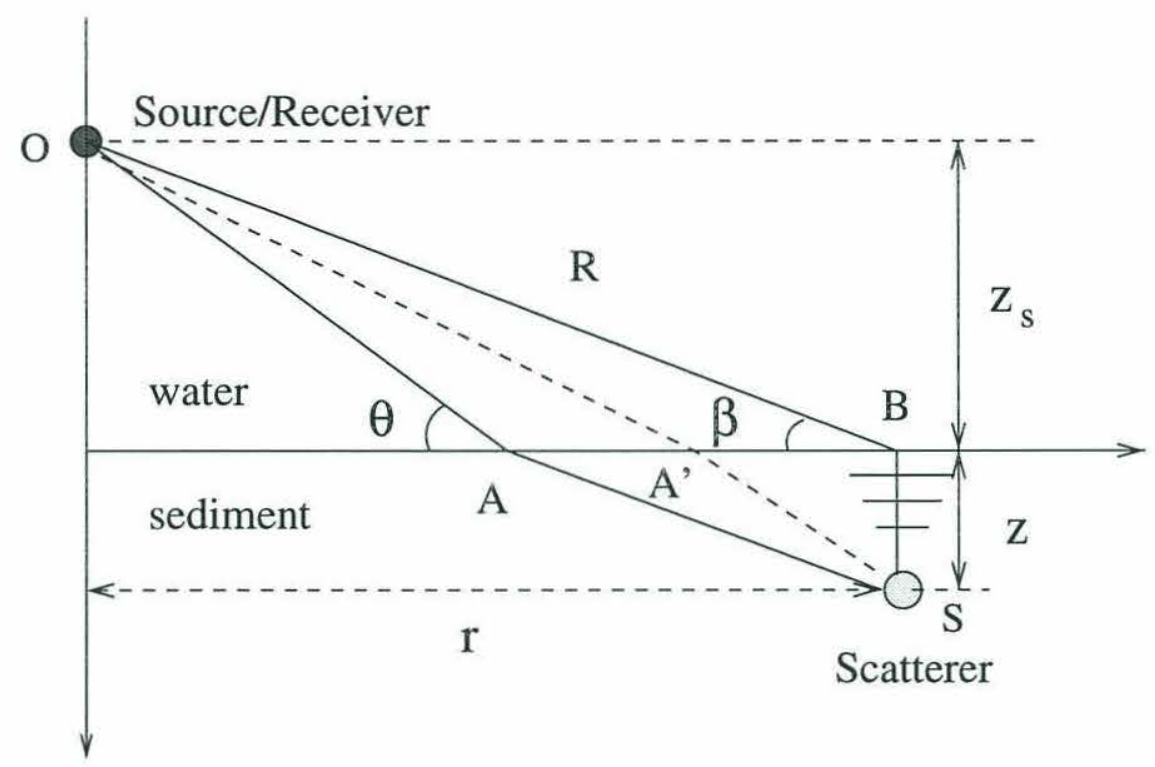

Figure 2-5: Schematic of scattering geometry for a two isovelocity half-space case.

the scatterer and then a plane wave traveling from point $B$ to the scatterer. For a slow bottom, there is only one eigenray path. Therefore, Path $O B S$ is used to approximate Path $O A^{\prime} S$. Point $B$ is the approximate stationary point and $A$ ' is the exact one; For a fast bottom, there are two cases. When the incident grazing angle $\alpha$ is larger than the critical angle, the situation is similar to that for a slow bottom. Only one eigenray path exists and $A$ is the exact stationary point. However, when $\alpha$ is smaller than the critical angle, both Path $O A S$ and $O B S$ will exist. Paths $O A S$ and $O B S$ correspond to the refracted wave and the the evanescent wave, respectively. If $\alpha$ is close to the critical angle, there might be more than two eigenrays according to Westwood [54]. On the one hand, point $B$ is not the same as point $A$ or $A$ ', which will bring in a certain degree of error for $\alpha$ larger than the critical angle. On the other hand, for $\alpha$ larger than the critical grazing angle, the stationary point $A$ contribution, i.e., the refracted wave, will be neglected. When the bottom attenuation is high and the scatterer is close to the water/sediment interface, this approach is justified, since the ray path of the refracted wave will be much longer than that of the lateral wave. The refracted wave would be greatly attenuated because of that. However, we will be able to see some differences if the above conditions cannot be satisfied. Mourad 
and Jackson's approach [1] is somewhat similar to Ivakin's. Instead of regarding the propagation as consisting of a spherical wave traveling from the source to the interface point, the incident wave is considered to be a plane or locally plane wave with incident direction allowed to vary over the insonified region in order to match the true incident direction. For convenience, we will call the above approach the far-field approximation in this work.

The formula that Hines derived in his paper [14], also a far-field approach, works for a two isovelocity half-space environment only. He applied the stationary phase method as well. In order to distinguish it from the above-mentioned far-field approximation, we will name it Hines' method in the later comparison. In a two isovelocity half-space situation, $g_{1}\left(k_{r}, z\right)$ in Eq.2.21 can be further expressed as

$$
g_{1}\left(k_{r}, z\right)=T_{w s} e^{i k_{z_{1}} z}
$$

where $T_{w s}$ is the transmission coefficient from the water to the sediment, $k_{z_{1}}$ is the vertical wavenumber in the sediment, and $z$ is the distance from the interface to the scatterer. If Eq.2.21 is rewritten in terms of the angles as plotted in Fig. 2-5 and includes the result in Eq.2.18, we will have

$$
G(r, z)=e^{i \frac{\pi}{4}} \sqrt{\frac{k_{0}}{2 \pi r}} \int_{i \infty-\pi / 2}^{-i \infty+\pi / 2} T_{w s}\left(\theta_{i}\right) \sqrt{\cos \theta_{i}} e^{i k_{0}\left(r \cos \theta_{i}-z_{s} \sin \theta_{i}+z \sqrt{\left.n_{w s}^{2}-\cos ^{2} \theta_{i}\right)}\right.} d \theta_{i}
$$

where $\theta_{i}$ is the incident angle and $n_{w s}=k_{1} / k_{0} . \quad k_{1}$ here represents the medium wavenumber in the sediment. Eq. 2.24 is a little different from that in Hines' paper since we choose $\theta_{i}$ to be the incident angle from the water to the sediment instead of the opposite. The phase of the integrand is now

$$
f\left(\theta_{i}\right)=i k_{0}\left(r \cos \theta_{i}-z_{s} \sin \theta_{i}+z \sqrt{n_{w s}^{2}-\cos ^{2} \theta_{i}}\right)
$$

$n_{w s}$ here can be complex to account for the attenuation in the sediment. According 
to the stationary phase method, the stationary point can be found from the following

$$
\frac{d f\left(\theta_{i}\right)}{d \theta_{i}}=-i k_{0}\left(r \sin \theta_{i}+z_{s} \cos \theta_{i}-\frac{z \sin \left(2 \theta_{i}\right)}{2 \sqrt{n_{w s}^{2}-\cos ^{2} \theta_{i}}}\right)=0
$$

There will be two solutions to Eq.2.26 for a fast bottom. The solution corresponding to the evanescent wave can be obtained by perturbing the incident angle $\beta$, where

$$
\beta=\tan ^{-1}\left(-z_{s} / r\right)
$$

The real solution can be found by performing a first-order Taylor expansion,

$$
\theta_{e}=\beta-\frac{f^{\prime}(\beta)}{f^{\prime \prime}(\beta)}
$$

where the prime (') represents the derivative with respect to the incident angle. We have to use a complex root finder to obtain the second solution $\theta_{r}$, which corresponds to the refracted wave. The perturbation method suggested by Hines has some difficulties when the actual stationary point is close to a singular point, the critical angle in this case. It might also be a problem for $\theta_{e}$ and is possibly a cause of the overestimate for the field in the region close to the critical angle as can be seen later.

For the two half-space, fast bottom situation, the incident spherical wave is therefore decomposed into two plane waves: the refracted wave and the evanescent wave. The evanescent wave exists only in the subcritical grazing angle region. The stationary phase method gives the results

$$
Q(\theta)=\frac{A(\theta)}{B(\theta)}
$$

where

$$
A(\theta)=T_{w s}(\theta) \sqrt{\cos \theta} e^{i k_{0}\left(r \cos \theta-z_{s} \sin \theta+z \sqrt{n_{w s}^{2}-\cos ^{2} \theta}\right)}
$$


and

$$
B(\theta)=\sqrt{r\left(r \cos \theta-z_{s} \sin \theta-z\left[\frac{\cos (2 \theta)}{\sqrt{n_{w s}^{2}-\cos ^{2} \theta}}-\frac{\sin ^{2}(2 \theta)}{4 \sqrt{\left(n_{w s}^{2}-\cos ^{2} \theta\right)^{3}}}\right]\right)},
$$

For the refracted wave

$$
G_{r}=Q\left(\theta_{r}\right)
$$

and for the evanescent wave

$$
G_{e}=Q\left(\theta_{e}\right)
$$

From the results in the next section, this method can be seen to be reasonably good for the two isovelocity half-space case, except in the region close to the critical angle, where there are more than two stationary points, as pointed out by Westwood [54]. However, it is not trivial to find all the stationary points for a bottom with multiple layers or with a sound speed gradient because the phase term of the integrand in Eq.2.19 becomes much more complicated.

\subsection{Comparison of Propagation Models}

The far-field approximation and Hines' method are widely implemented in bottom scattering modeling, especially in high-frequency problems. Yet their validity and limitations need to be recognized when dealing with general experimental scenarios. In this section, based on real geoacoustic parameters, the above two propagation models will be evaluated through comparisons with exact solutions obtained by numerical wavenumber integrations. An effort will also be made to identify their possible influence on the quantification of bottom scattering.

We will concentrate on three types of fluid models [cf. Fig. 2-6]: two isovelocity half-spaces; two half-spaces with an upward-refracting sound speed profile in the sediment; a layer with an upward-refracting profile between two isovelocity half-spaces. 


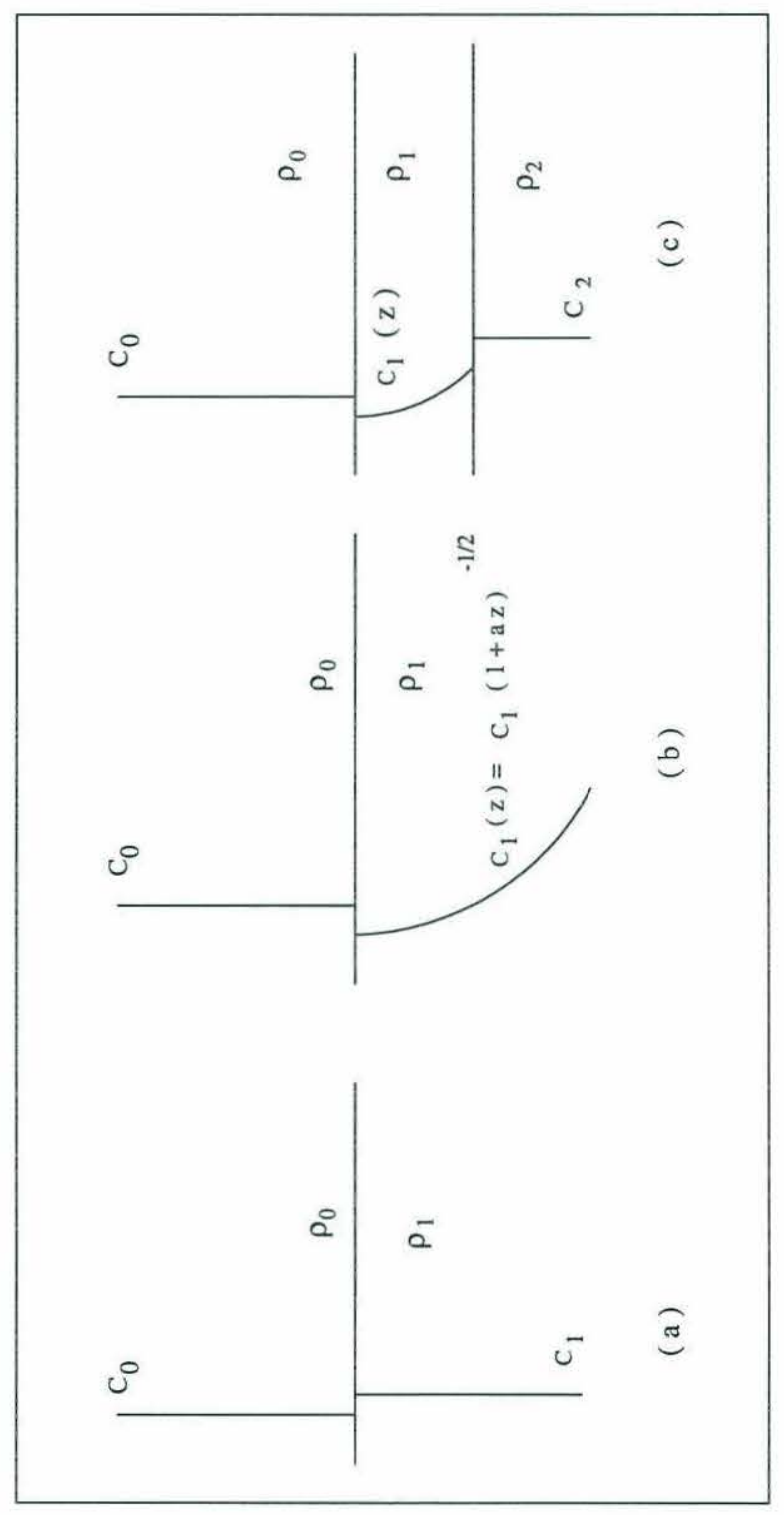

Figure 2-6: The sound speed profile for three types of bottom: (a) two isovelocity half spaces; (b) two half spaces with a sound speed gradient in the bottom; (c) three layers with a sound speed gradient in the middle layer. 
Most of the geoacoustic parameters used in the comparisons are measurement results or extrapolations of measured data which have appeared in the literature. In this section, $f$ will stand for frequency, $c_{i}$ and $\rho_{i}$ will stand for the sound speed and density in the overlying water $(i=0)$, just beneath the water/sediment interface $(i=1)$, and in the lower half space $(i=2)$ in Fig. 2-6(c). The density is chosen to be constant in each layer. The loss factor $\delta_{i},(i=0,1,2)$ is the ratio of the imaginary and real parts of the complex wavenumber at the top of each sediment layer. The quantity $g$ represents the sound speed gradient, and $H$ stands for the middle layer thickness in the three-layer case. The wavelength in the water is given by $\lambda$, and $z_{s}$ is the source height above the water/bottom interface and $z$ is the distance between the scatterer in the bottom and the water/sediment interface. The quantity $z_{s}$ and $z$ are scaled relative to wavelength $\lambda$. The source height $z_{s}$ is chosen to be $100 \lambda$ in order to avoid the near-field effect, which is true for the whole section. The transmission loss in the comparisons is defined as the ratio in decibels between the acoustic pressure at the scatterer for a simple point source and the pressure produced at a distance of $1 \mathrm{~m}$ from the same source.

\subsubsection{Two Isovelocity Half-Spaces}

The sound speed in the sediment depends on the sediment type. The sediment/water sound speed ratio could be larger or smaller than 1, which determines the existence of a critical angle. According to Hamilton [58], for soft sediments such as silty clay, the sound speed just beneath the water/sediment interface is usually less than that of the overlying water. The geoacoustic parameters used in Fig. 2-7, which is consistent with Continental Rise data and taken from Fig. 3 in Mourad and Jackson's paper [1], is an example. From the field at scatterers situated at $z=0$ and $z=-2 \lambda$, it can be seen that results of the far-field approximation and Hines' method are almost identical to that of the numerical wavenumber integration. There is no critical angle and only one stationary point, i.e., scatterers are insonified by the refracted wave only. Both approaches are good approximations in this situation. One would expect 

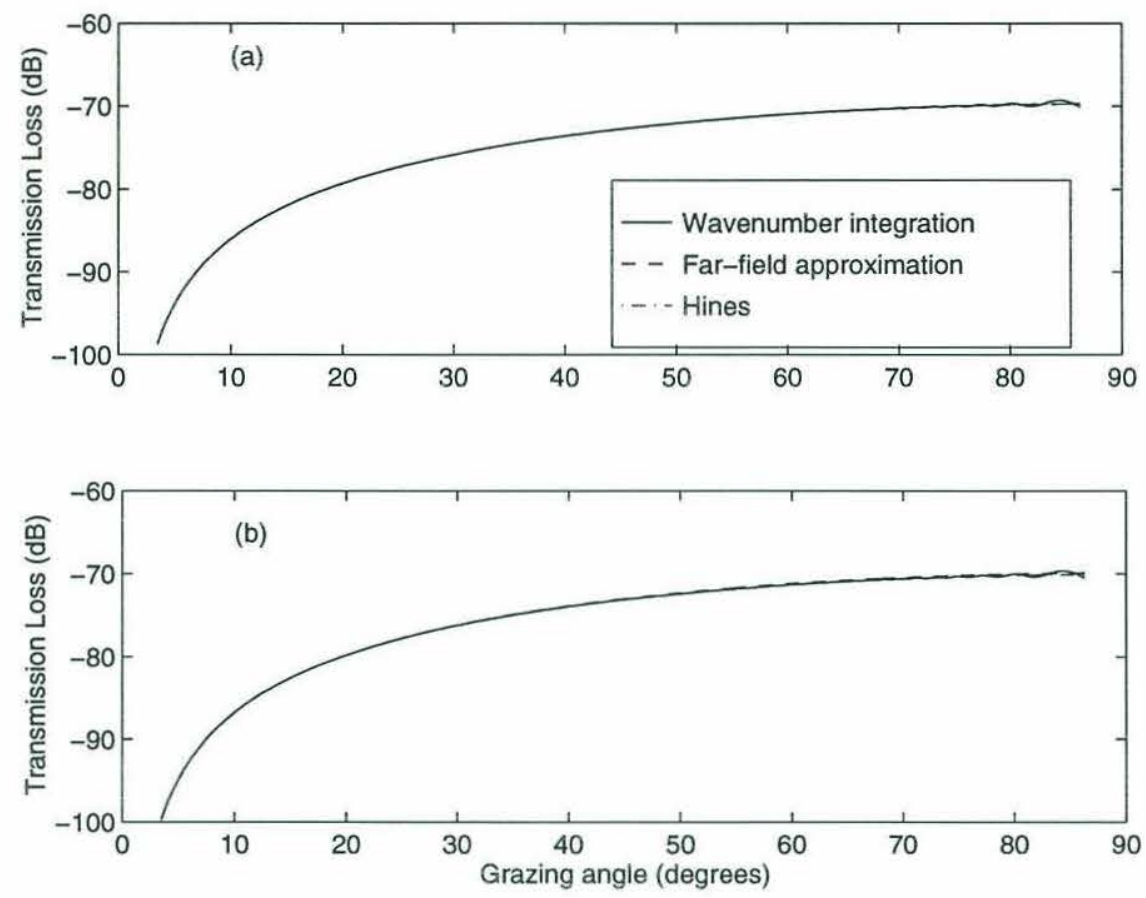

Figure 2-7: Comparison of transmission loss in the environment with no critical angle. Parameters are: $f=500 \mathrm{~Hz}, c_{0}=1530 \mathrm{~m} / \mathrm{s}, c_{1}=1510 \mathrm{~m} / \mathrm{s}, \rho_{1}=1.72 \mathrm{~g} / \mathrm{cm}^{3}$, $\delta=0.00164, z_{s}=100 \lambda$. The transmitted field at (a) $z=0$; (b) $z=-2 \lambda$.

the calculation of the equivalent surface scattering strength to be appropriate and accurate without including the multipath effect. Since $z_{s}$ and $z$ are both scaled relative to $\lambda$, the results obtained here and for the two isovelocity half-space case should be frequency-independent if the bottom attenuation is linear with frequency, which is an open question $[59,60,32]$.

For sandy bottoms and turbidite bottoms in certain areas, the sediment/water sound speed ratio is larger than 1. Input parameters in Fig. 2-8 correspond to the Hudson Canyon experiment reported by Rogers et al. [61], where the bottom is described as silty sand and silty clay. We choose the frequency to be $10 \mathrm{kHz}$ and assign the bottom attenuation to be $0.1 \mathrm{~dB} / \mathrm{m} / \mathrm{kHz}\left(\delta_{i}=0.0027\right)$, which is at the high end for this type of bottom. The transmitted field is again shown at different depths in Fig. 2-8. It is found that the agreement between Hines' method and the numerical wavenumber integration technique is fairly good except for some overestimate in the 

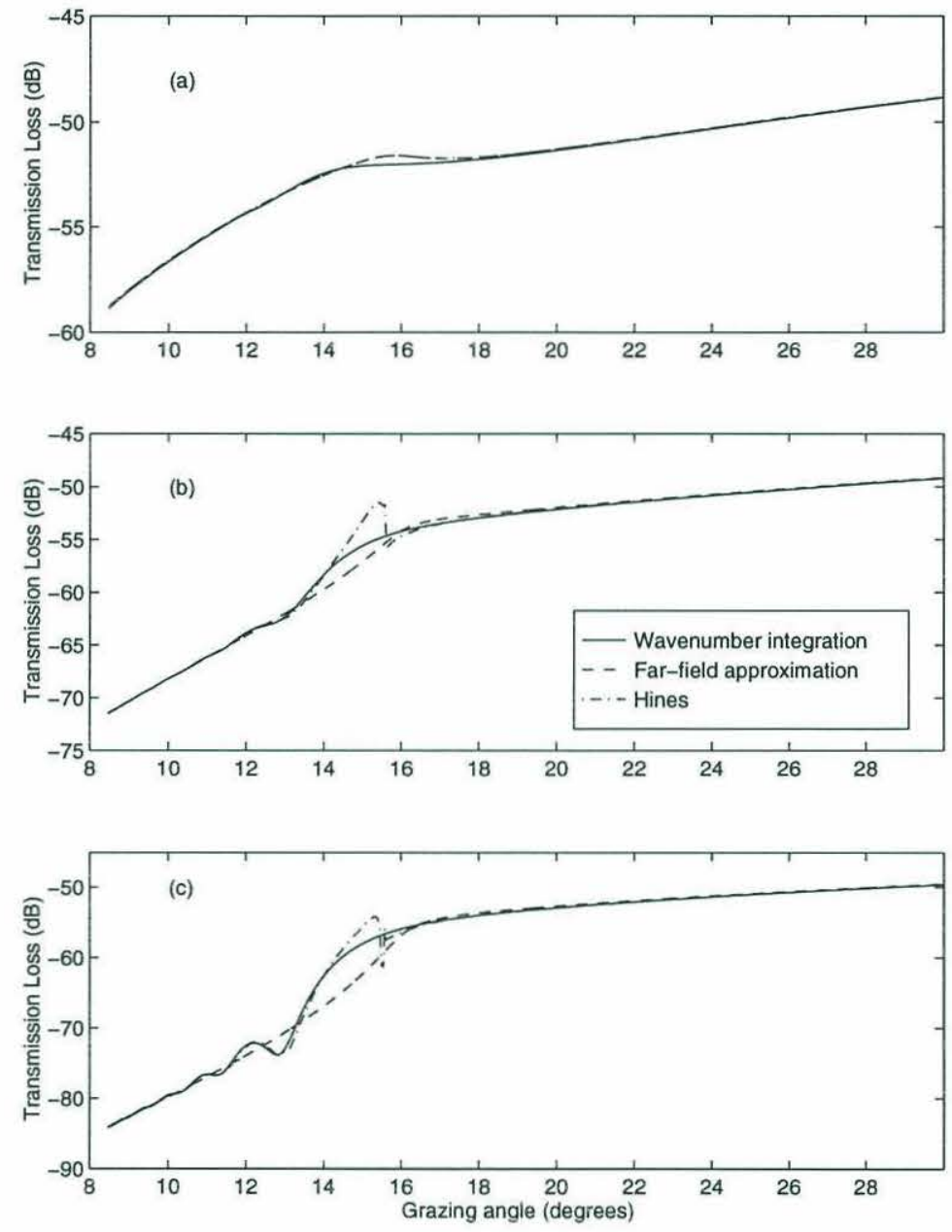

Figure 2-8: Comparison of transmission loss with the critical angle effect for a low attenuation bottom. Parameters are: $f=500 \mathrm{~Hz}, c_{0}=1500 \mathrm{~m} / \mathrm{s}, c_{1}=1560 \mathrm{~m} / \mathrm{s}$, $\rho_{1}=1.8 \mathrm{~g} / \mathrm{cm}^{3}, \delta=0.0027, z_{s}=100 \lambda$. The transmitted field at (a) $z=0$; (b) $z=-\lambda$; (c) $z=-2 \lambda$. 


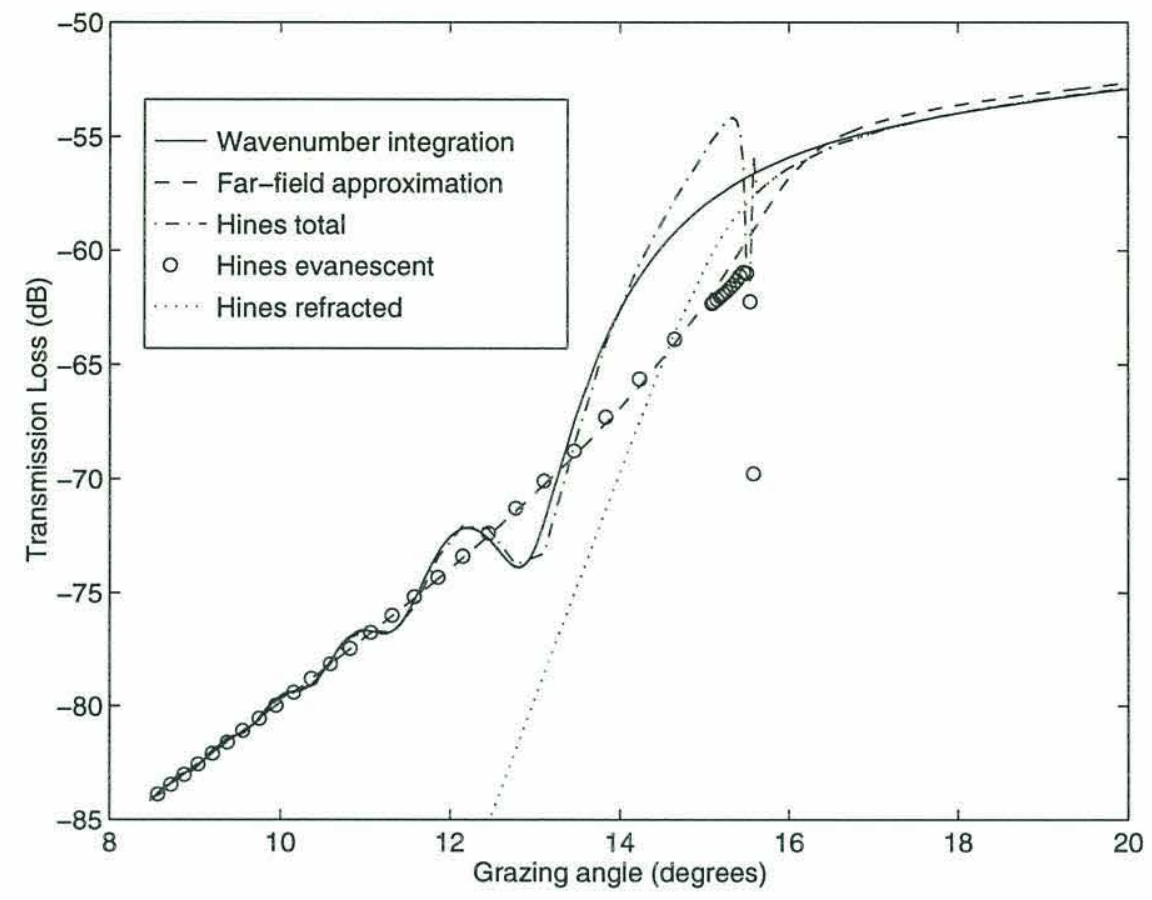

Figure 2-9: The detailed comparison. Parameters are the same as that in Fig. 2-8(c).

region just below the critical grazing angle. The critical angle $\left(\theta_{c}=\cos ^{-1}\left(c_{0} / c_{1}\right)\right)$ is 16 degrees and is indicated in the figure. The far-field approximation, on the other hand, tends to underestimate the field at subcritical grazing angles. Differences will increase with the depth of the scatterer [cf. Fig. 2-8]. In Fig. 2-9, we add the evanescent wave and refracted wave contributions to Fig. 2-8(c). For the far-field approximation, it is clear that the neglect of the refracted wave in the subcritical angle region is the cause of the underestimate. The small error before the critical angle can be attributed to the difference between the true stationary point and that used in the approximation. As for Hines' method, the match is excellent everywhere except in a small region after the critical angle. This is the transition area from a field composed of one stationary point to a field composed of two stationary points, where the approximation would normally be invalid, according to Westwood [54]. Another possible cause might be the failure of the first-order perturbation method to find the stationary point close to the singularity associated with the critical angle. 

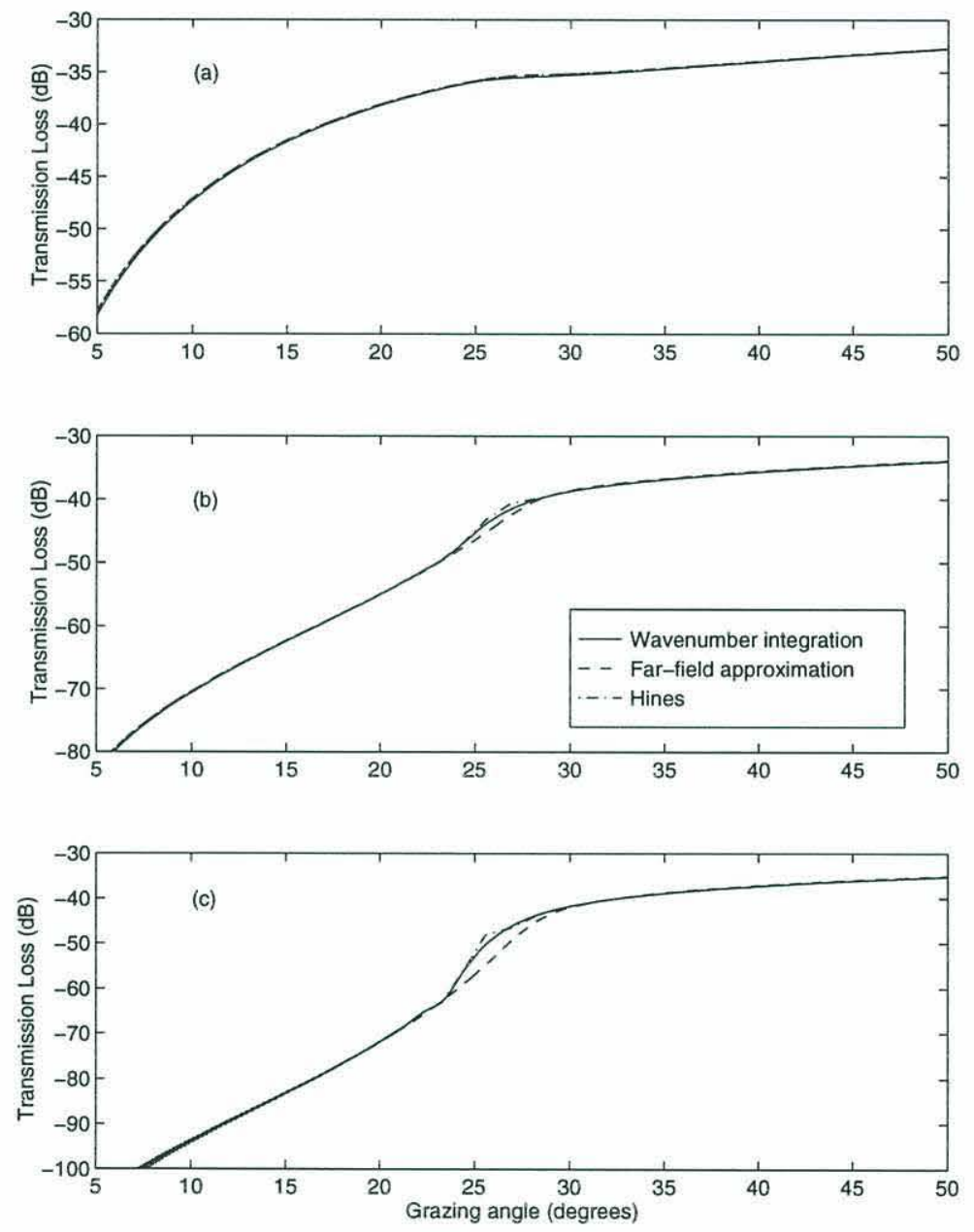

Figure 2-10: The critical angle effect for a high attenuation bottom. Parameters are: $f=40000 \mathrm{~Hz}, c_{0}=1500 \mathrm{~m} / \mathrm{s}, c_{1}=1689 \mathrm{~m} / \mathrm{s}, \rho_{1}=1.97 \mathrm{~g} / \mathrm{cm}^{3}, \delta=0.0166$, $z_{s}=100 \lambda$. The transmitted field at (a) $z=0$; (b) $z=-\lambda$; (c) $z=-2 \lambda$. 
For a sandy bottom, the attenuation will be much higher than that for a soft bottom. The geoacoustic parameters in Fig. 2-10 are from the CBBL Panama City site [18]. Frequency of $40 \mathrm{kHz}$ is chosen, the same as that in the experiment. It is a sandy bottom and the attenuation was found to be $0.524 \mathrm{~dB} / \mathrm{m} / \mathrm{kHz}$, which is equivalent to $\delta_{i}=0.0166$. The critical angle is now 27.4 degrees. The figure shows results similar to those in Fig. 2-8 for the field at three different depths. Nevertheless, the difference between the far-field approximation and the numerical wavenumber integration is smaller than that in the lower attenuation bottom. The interference between the refracted wave and the evanescent wave is hardly noticeable at subcritical angles. The explanation for this is that the refracted wave has a longer ray path than that of the evanescent wave and is therefore greatly attenuated at long ranges in the highattenuation bottom. In other words, the evanescent wave contribution will prevail at subcritical angles. So we see from the above that the single stationary point treatment in the far-field approximation works better in a high-attenuation bottom than in a low-attenuation one.

The effect of different propagation models on the estimation of scattering strength estimation is of considerable interest in bottom scattering modeling. In Figs. 2-11 and 2-12, we have calculated the equivalent surface scattering strength [cf. Eq.2.6] associated with volume backscattering using the same parameters as those in Figs. 2-8 and 2-10. Point scatterers are assumed here for simplicity, and the volume scattering cross section $\sigma_{v}$ is chosen to be 0.00003 in Fig. 2-11 and 0.000906 in Fig. 2-12. Both in the ARSRP low-frequency sediment scattering experiment and the CBBL high-frequency scattering experiment at the Eckernfoerde Bay site, strong scattering layers are found to be a certain distance away from the water/sediment interface $[9,6]$. So we will choose a scattering layer with a thickness of $50 \lambda$ here starting from different depths below the interface. Figures 2-11, 2-12(b)(d)(f) show the difference in backscattering strength between the exact solution and the far-field approximation result. Since results from Hines' method fit exact solutions very well, but in a very narrow region, we consider that it would yield similar outputs and will not discuss it 

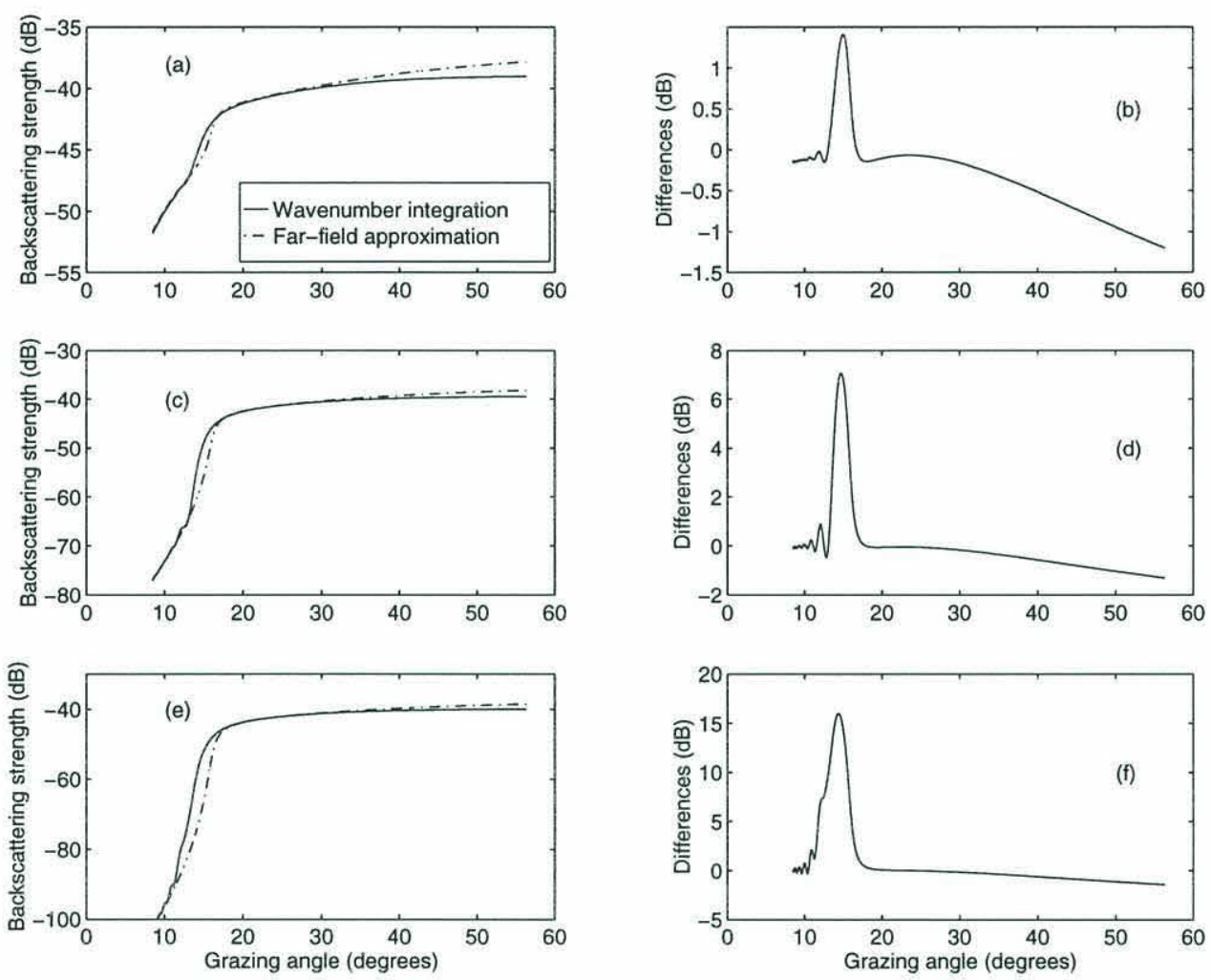

Figure 2-11: The equivalent surface backscattering strength for a low attenuation bottom assuming point scatterers. Parameters are the same as that in Fig. 2-8 and $\sigma_{v}=0.00003$. The backscattering strength and the difference as the scattering layer begins at (a)(b) $z=0 ;(\mathrm{c})(\mathrm{d}) z=-\lambda$; (e)(f) $z=-2 \lambda$. 

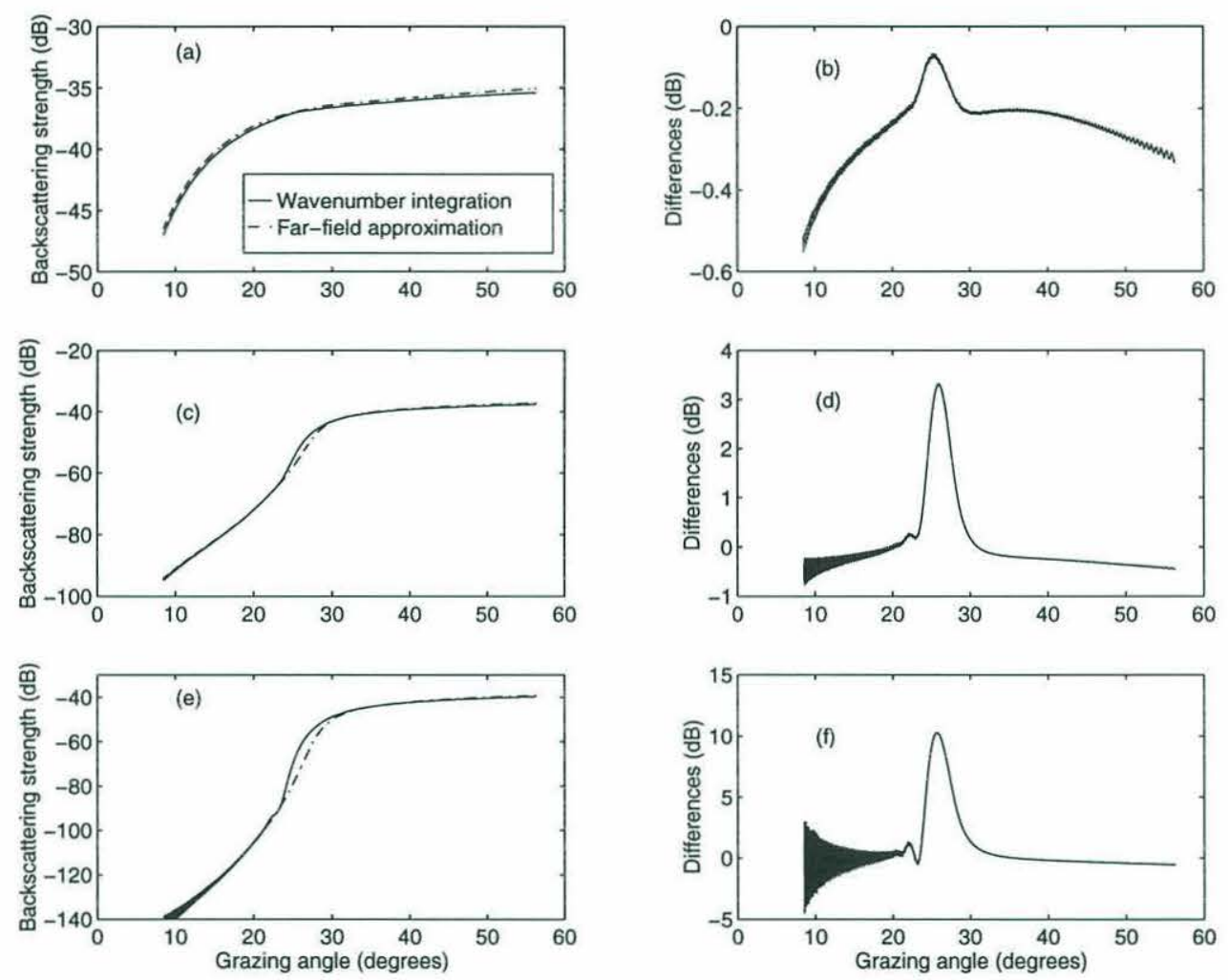

Figure 2-12: The equivalent surface backscattering strength for a high attenuation bottom assuming point scatterers. Parameters are the same as that in Fig. 2-10 and $\sigma_{v}=0.000906$. The backscattering strength and the difference as the scattering layer begins at (a)(b) $z=0 ;(\mathrm{c})(\mathrm{d}) z=-\lambda$; (e)(f) $z=-2 \lambda$. 
here.

From Fig. 2-11, it is evident that the underestimate of the field at subcritical angles for the far-field approximation leads to differences in equivalent surface backscattering strength. However, the biggest difference is only about $1 d B$ when the scattering layer starts right at the interface. On the other hand, when the scattering layer begins at greater depths, the difference is larger. For the scattering layer starting at two wavelengths beneath the interface, up to $15 d B$ difference appears. Also, we notice that the actual level of the backscattering strength in this case is only several $d B$ lower than that for the scattering layer starting from the interface. Therefore one can hardly distinguish them from the scattering measurement without a priori knowledge, which highlights the necessary precautions in scattering modeling using the far-field approximation in this environment. When the bottom attenuation is low, the penetration is deep. The multipath effect is inevitable. The scattered returns will reach the receiver via different paths, therefore different scattering angles, which makes the angular dependence of the scattering strength less meaningful. All of these results cast some doubt on the validity of using the equivalent surface scattering strength to characterize the volume scattering process.

Fig. 2-12 shows similar results for a sandy bottom. The difference in backscattering strength is smaller when compared with that in Fig. 2-11. Numerical errors can even be seen in Fig. 2-12(e) and (f). The scattering level for a scattering layer deep in the sediment is much lower than that at the interface. Since the sand is usually a strong scatterer and the bottom penetration is much shallower due to high attenuation, the problems that are addressed regarding Fig. 2-11 are much less serious. Both the far-field approximation and the equivalent surface scattering strength are appropriate. 


\subsubsection{Two Half-Spaces with Sound Speed Gradient in the Lower Half}

Sound speed gradients exist in both the water column and sediment in real ocean environments [62] and have significant effects on sound propagation. The gradient in the water column is generally small compared with that in the sediment and will be neglected. We will therefore focus on the effect of the sound speed gradient in the sediment. We will not include Hines' method in the comparisons from now on due to the complexity of extending it to complicated sound speed structures.

In order to obtain an analytic solution, we choose the $1 / c^{2}$ linear sound speed profile,

$$
c_{1}(z)=c_{1}(1-a z)^{-1 / 2},
$$

for a real constant $a$, with units of inverse length. The sound speed gradient at the interface is

$$
g=\left.\frac{d c_{1}(z)}{d z}\right|_{z=0}=\frac{c_{1} a}{2}
$$

Figure 2-13 shows the frequency dependence of the comparison between the farfield approximation and the numerical wavenumber integration for the case where there is a drop in sound speed at the water/sediment interface. The sound speed gradient $g$ has a typical value $1.0 \mathrm{~s}^{-1}$. The mismatch of the oscillation pattern is obvious at $100 \mathrm{~Hz}$, but as the frequency increases, the match is better. At $500 \mathrm{~Hz}$, they are almost indistinguishable. The oscillation here comes from the interference between the direct incident wave and the upward-refracted wave. The sound speed gradient will determine the path length of the upward refracting ray. At high frequencies, the refracted wave will be attenuated so much that virtually no interference occurs. Therefore the higher the frequency, the better the far-field approximation. The elucidation of the mismatch in oscillation patterns rests on the examination of the refracted wave. In the far-field approximation, the field is dominated by the interference between the plane wave incident at a particular angle and the refracted 

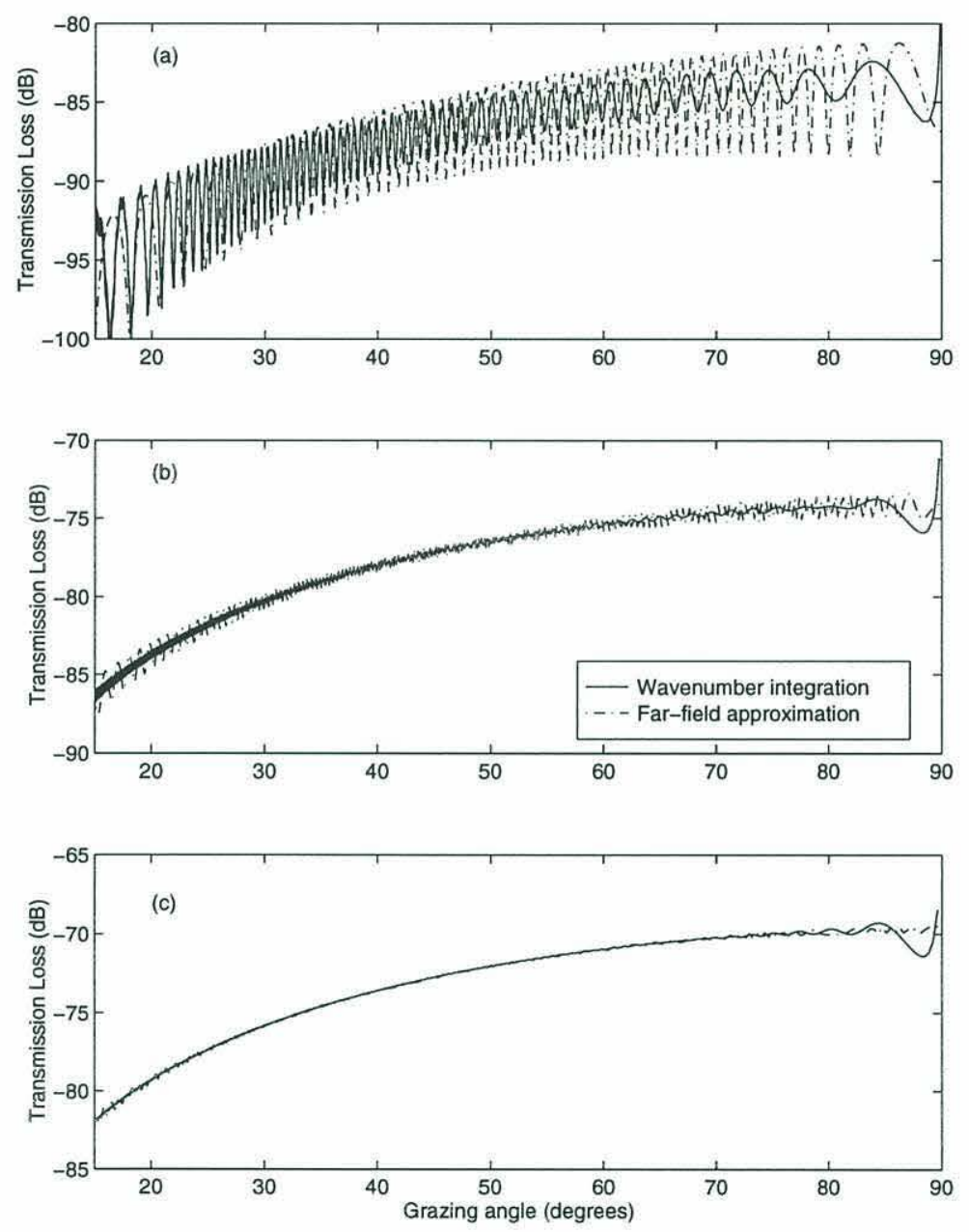

Figure 2-13: The frequency dependence of the comparison results. Parameters are: $c_{0}=1530 \mathrm{~m} / \mathrm{s}, c_{1}=1510 \mathrm{~m} / \mathrm{s}, \rho_{1}=1.72 \mathrm{~g} / \mathrm{cm}^{3}, \delta=0.00164, \mathrm{~g}=1 \mathrm{~s}^{-1}, z_{s}=100 \lambda$, $z=0$ and (a) $f=100 \mathrm{~Hz}$; (b) $f=300 \mathrm{~Hz}$; (c) $f=500 \mathrm{~Hz}$. 
wave originating from the same angle of incidence. While in the numerical wavenumber integration approach, the interference involves refracted waves originating from many incident angles. It all boils down to the plane wave assumption in the far-field approximation, which is not included in the wavenumber integration approach.

The parameters in Fig. 2-14 correspond to the case where there is an increase in sound speed at the water/sediment interface. The gradient $g$ is again chosen to be $1.0 \mathrm{~s}^{-1}$. Fig. 14 shows the field for three different frequencies at two different depths. As we anticipated, the field now includes the contribution from the evanescent wave, the interface refracted wave and the turning refracted wave, as shown in Fig. 2-15. Together, they will determine the interference pattern. The critical angle effect is still clear [cf. Sec. 2.4.1]. At higher frequencies, the results approach those for the two isovelocity half-space case because the turning refracted wave is strongly attenuated. All in all, the far-field approximation will have some problems at low frequency for this kind of low-attenuation bottom with a sound speed gradient. As for the high attenuation sandy bottom, we would expect similar phenomena as those at high frequency in Fig. 2-14, as can be seen in Fig. 2-16. The parameters in Fig. 2-16 are the same as in for Fig. 2-10 except that the frequency is $100 \mathrm{~Hz}$. Also, we have studied the effect of the sound speed gradient. The gradient will decide the path length for the turning refracted wave, therefore the amplitude, in a bottom with attenuation. We would expect higher amplitude for the turning refracted wave in a bottom with a larger gradient, because the path length would be shorter. As a result, the oscillation is more significant for a larger gradient as shown in Fig. 2-17.

Again we will study the influence of the propagation model on the quantification of the scattering process. We still assume point scatterers here and calculate only the backscattering strength. The scattering layer will be considered starting from the water/sediment interface only. For a high-attenuation sandy bottom like that used to generate Fig. 2-16, we choose $\sigma_{v}$ to be 0.000906 . As shown in Fig. 2-18, the equivalent surface backscattering strength calculated with the far-field approxima- 

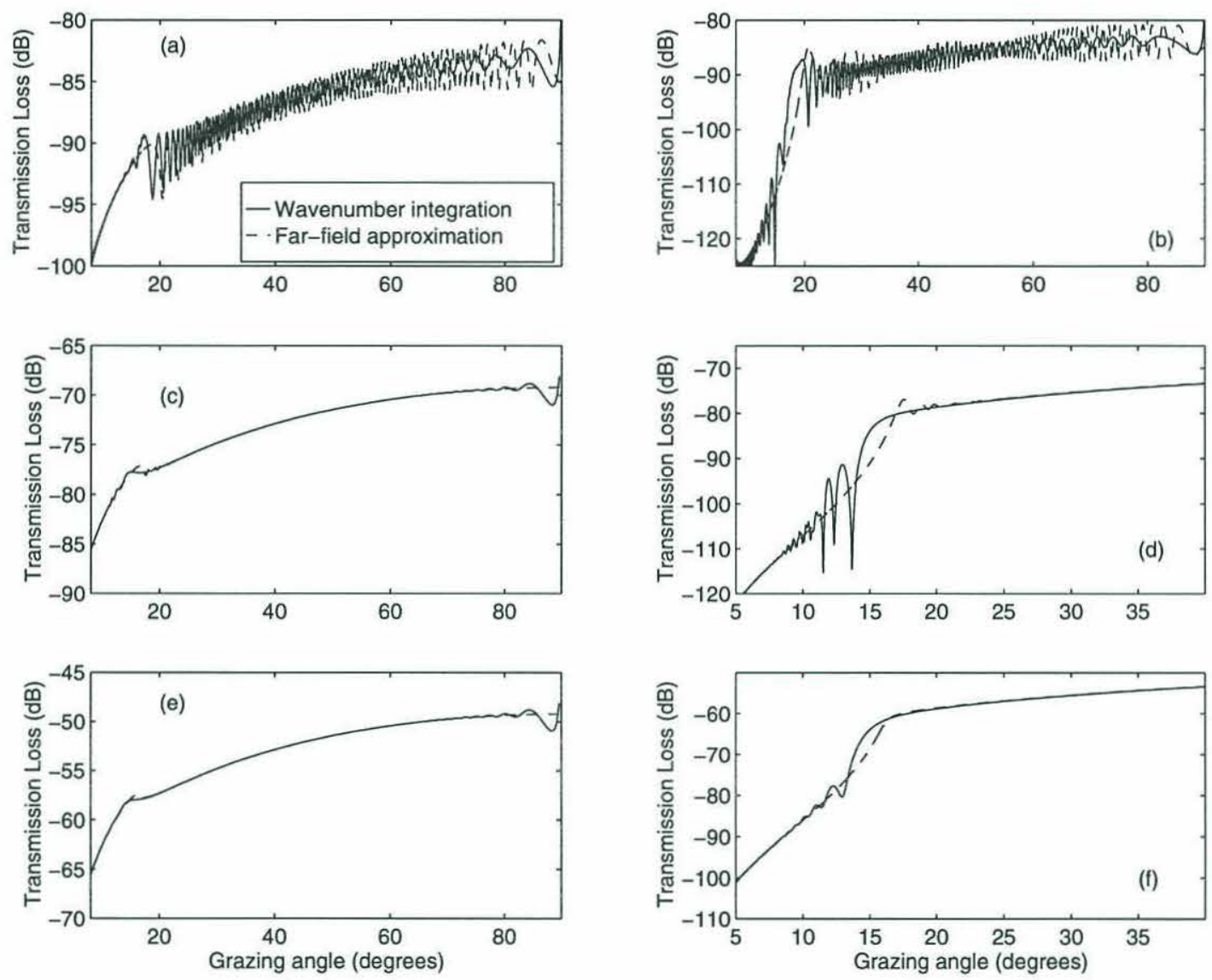

Figure 2-14: The frequency and depth dependence of the comparison results for a low attenuation bottom with a critical angle. Parameters are $c_{0}=1500 \mathrm{~m} / \mathrm{s}, c_{1}=$ $1560 \mathrm{~m} / \mathrm{s}, \rho_{1}=1.8 \mathrm{~g} / \mathrm{cm}^{3}, \delta=0.0027, \mathrm{~g}=1 \mathrm{~s}^{-1}, z_{s}=100 \lambda$ and (a) $f=100 \mathrm{~Hz}, z=0$ ; (b) $f=100 \mathrm{~Hz}, z=-2 \lambda$; (c) $f=500 \mathrm{~Hz}, z=0$; (d) $f=500 \mathrm{~Hz}, z=-2 \lambda$; (e) $f=5000 \mathrm{~Hz}, z=0$;(f) $f=5000 \mathrm{~Hz}, z=-2 \lambda$. 


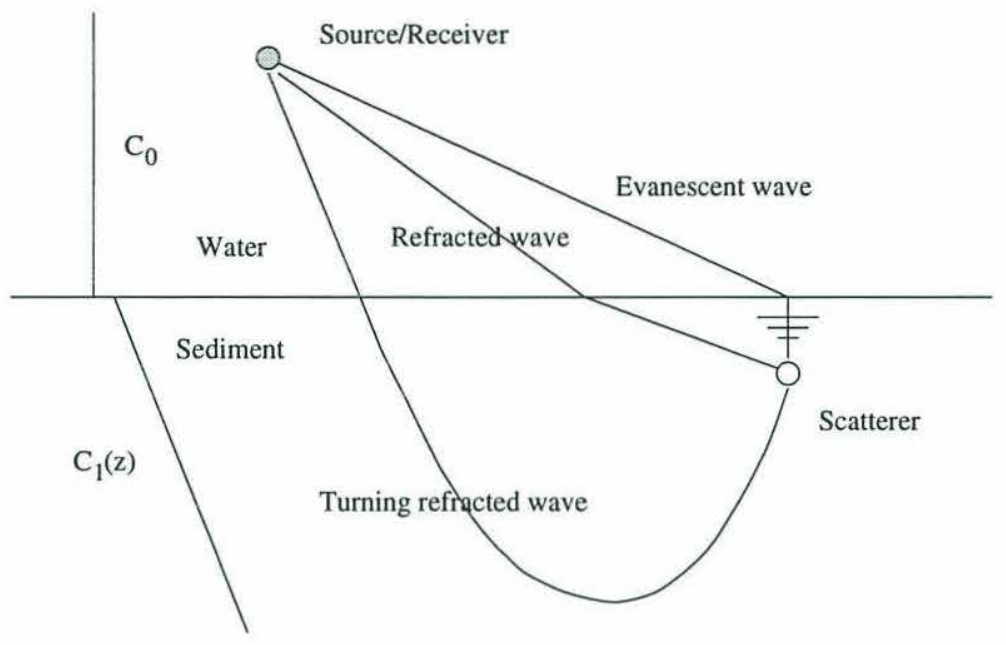

Figure 2-15: The propagation paths for two half-space fast bottom with an upwardrefracting sound speed profile in the bottom.
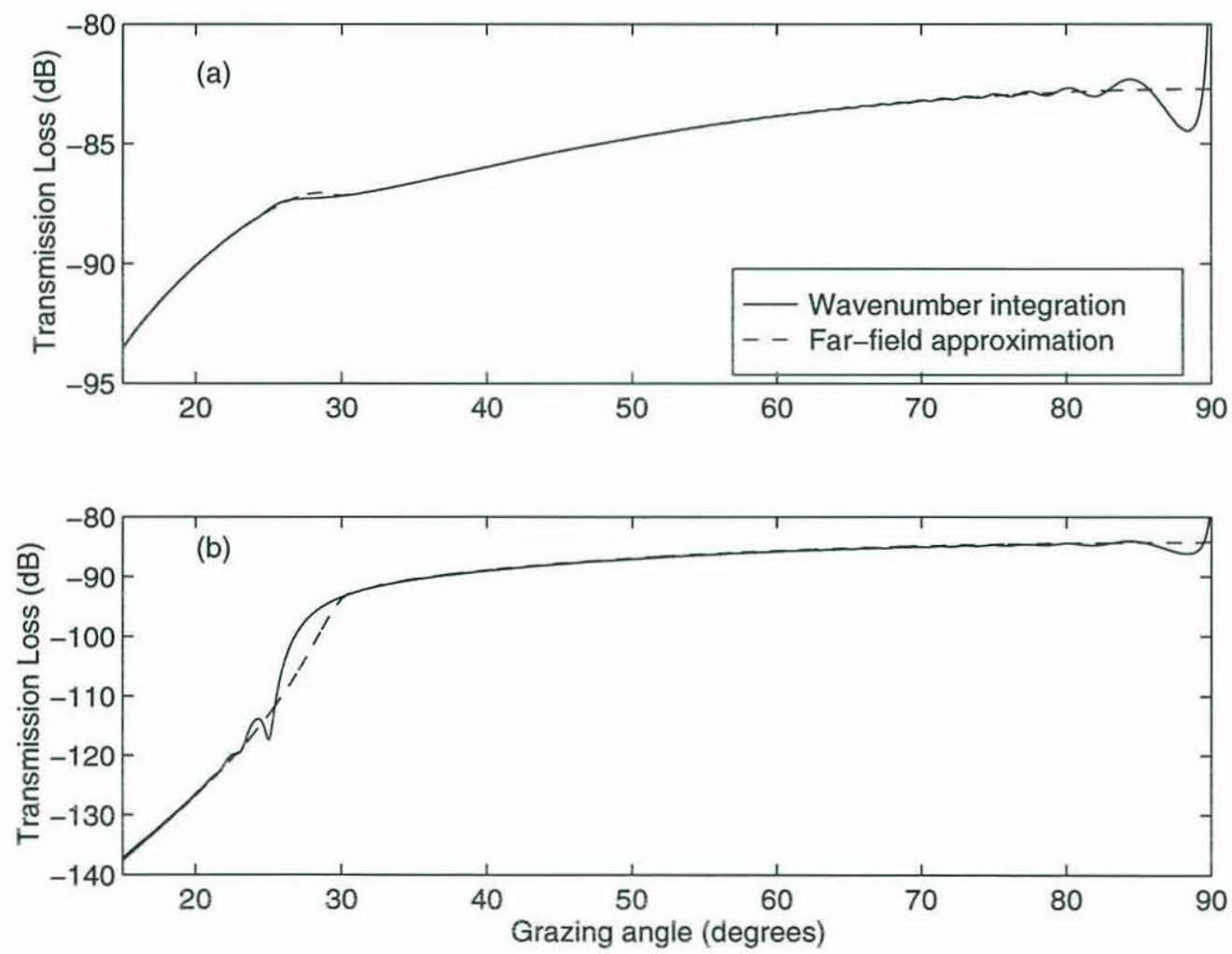

Figure 2-16: The depth dependence for a high-attenuation bottom. Parameters are: $f=100 \mathrm{~Hz}, c_{0}=1500 \mathrm{~m} / \mathrm{s}, c_{1}=1689 \mathrm{~m} / \mathrm{s}, \rho_{1}=1.97 \mathrm{~g} / \mathrm{cm}^{3}, \delta=0.0166, \mathrm{~g}=1 \mathrm{~s}^{-1}$, $z_{s}=100 \lambda$. The transmitted field at (a) $z=0$; (b) $z=-2 \lambda$. 

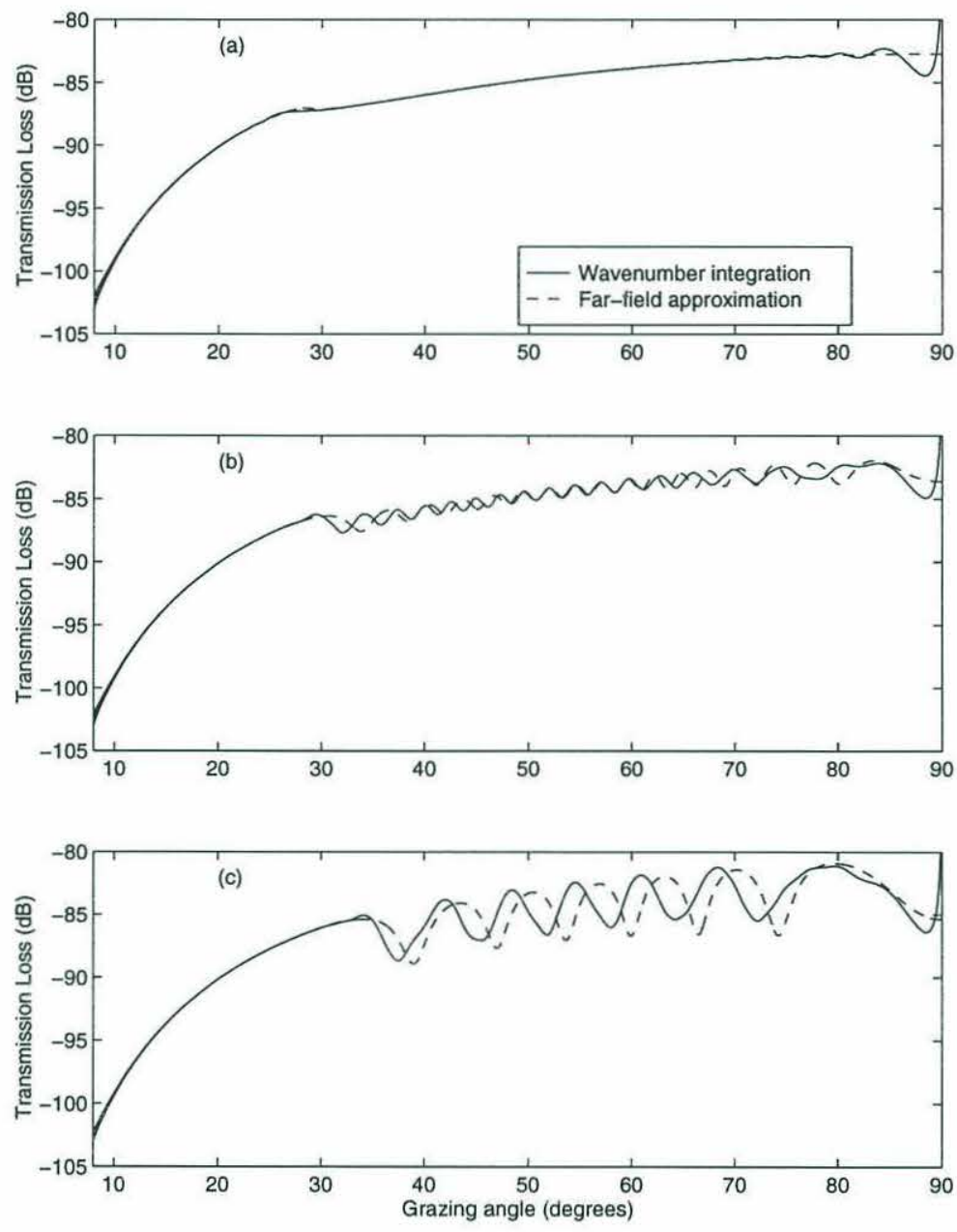

Figure 2-17: The sound speed gradient dependence. Parameters are the same as that in Fig. 2-13(a) except the gradient (a) $g=1 s^{-1}$; (b) $g=4 s^{-1}$; (c) $g=10 s^{-1}$. 

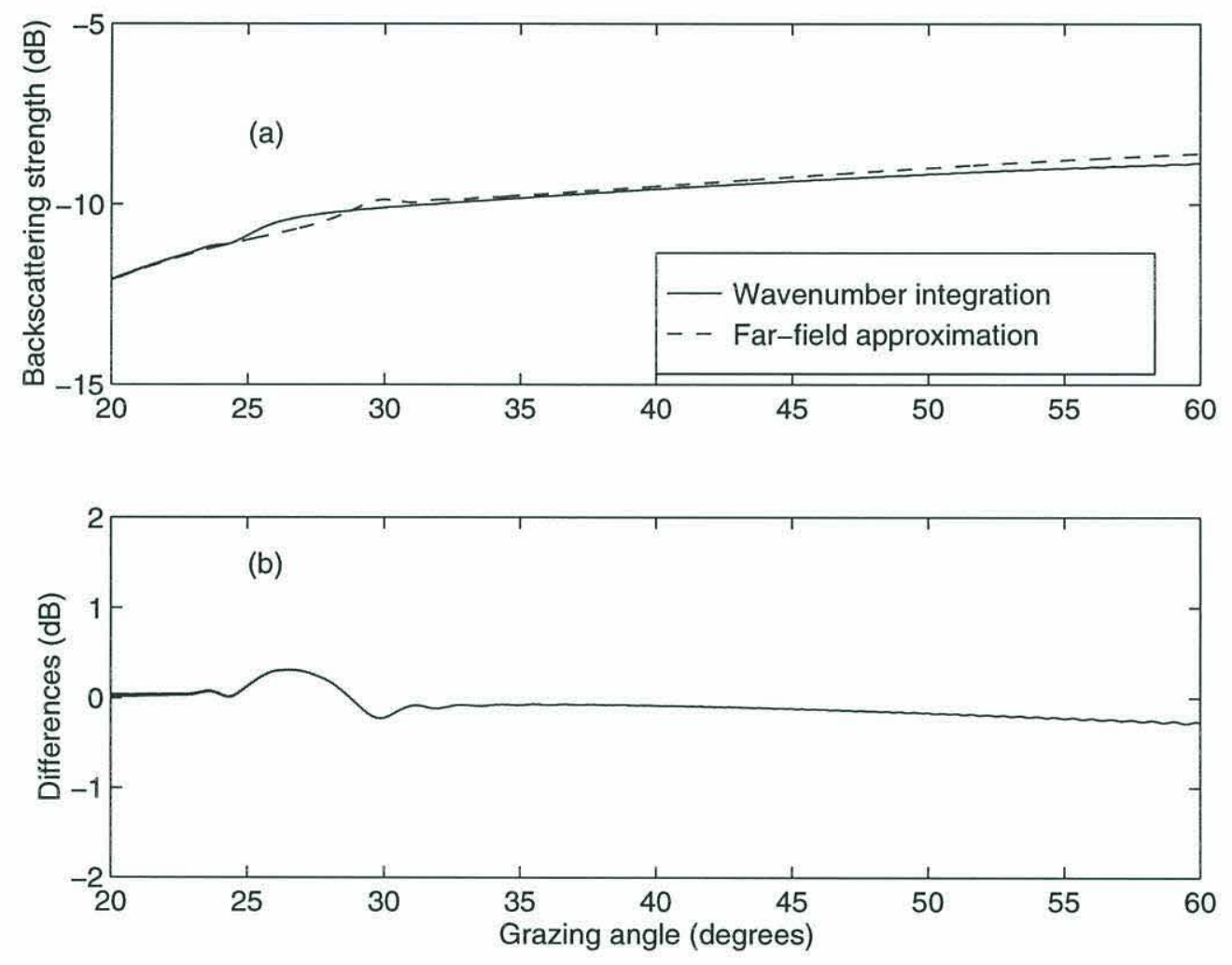

Figure 2-18: The equivalent surface backscattering strength for a high-attenuation bottom assuming point scatterers. Parameters are the same as that in Fig. 2-16 with the starting depth of the scattering layer to be at $z=0$. (a) the backscattering strength; (b) differences. 

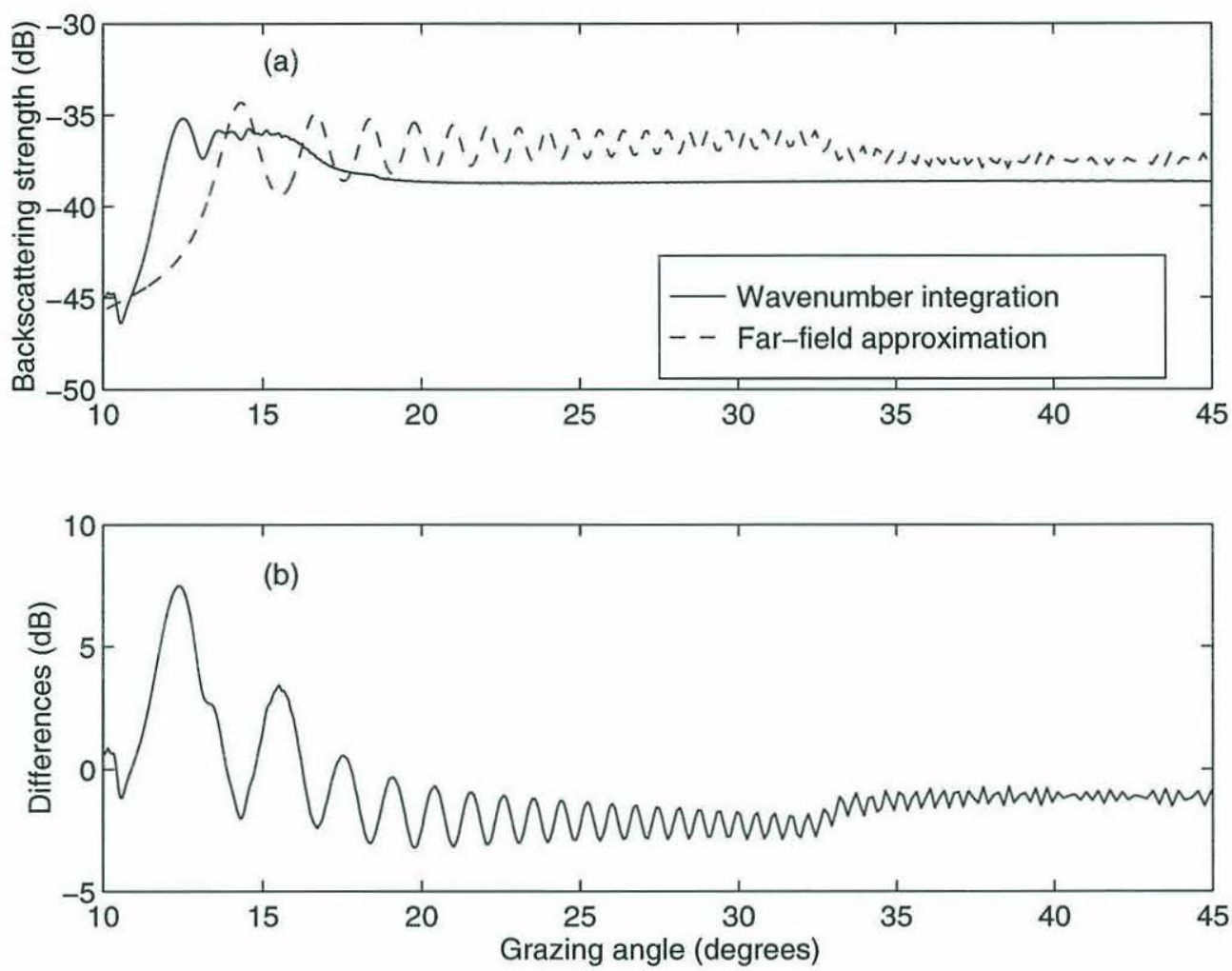

Figure 2-19: The equivalent surface backscattering strength for a low-attenuation bottom assuming point scatterers. Parameters are: $f=2300 \mathrm{~Hz}, c_{0}=1550 \mathrm{~m} / \mathrm{s}$, $c_{1}=1576 \mathrm{~m} / \mathrm{s}, \rho_{1}=1.83 \mathrm{~g} / \mathrm{cm}^{3}, \delta=0.00193, \mathrm{~g}=10 \mathrm{~s}^{-1}, z_{s}=100 \lambda$. The scattering layer starts at $z=0$. (a) the backscattering strength; (b) differences.

tion is very close to the exact solution. For a low-attenuation, soft sediment bottom, we let the geoacoustic input be the same as that for Fig. 15 (b) in Mourad and Jackson's paper [1]. Fig. 2-19 shows the backscattering strength predicted by the far-field approximation and the numerical wavenumber integration technique. Notice that the oscillations are absent for the numerical solution but still present for the farfield approximation. An interesting phenomenon appearing in the results obtained by numerical wavenumber integration is that the backscattering strength has a drop of about $3 d B$ at 16 degrees, which is not shown in the results when the far-field approximation is applied. In Mourad and Jackson's figure 15(b), the experimental data also shows an $8-10 d B$ fall. Although the drop in our prediction does not match the data exactly, it is one step closer and suggests that the far-field approximation may not be good enough in situations like this. 


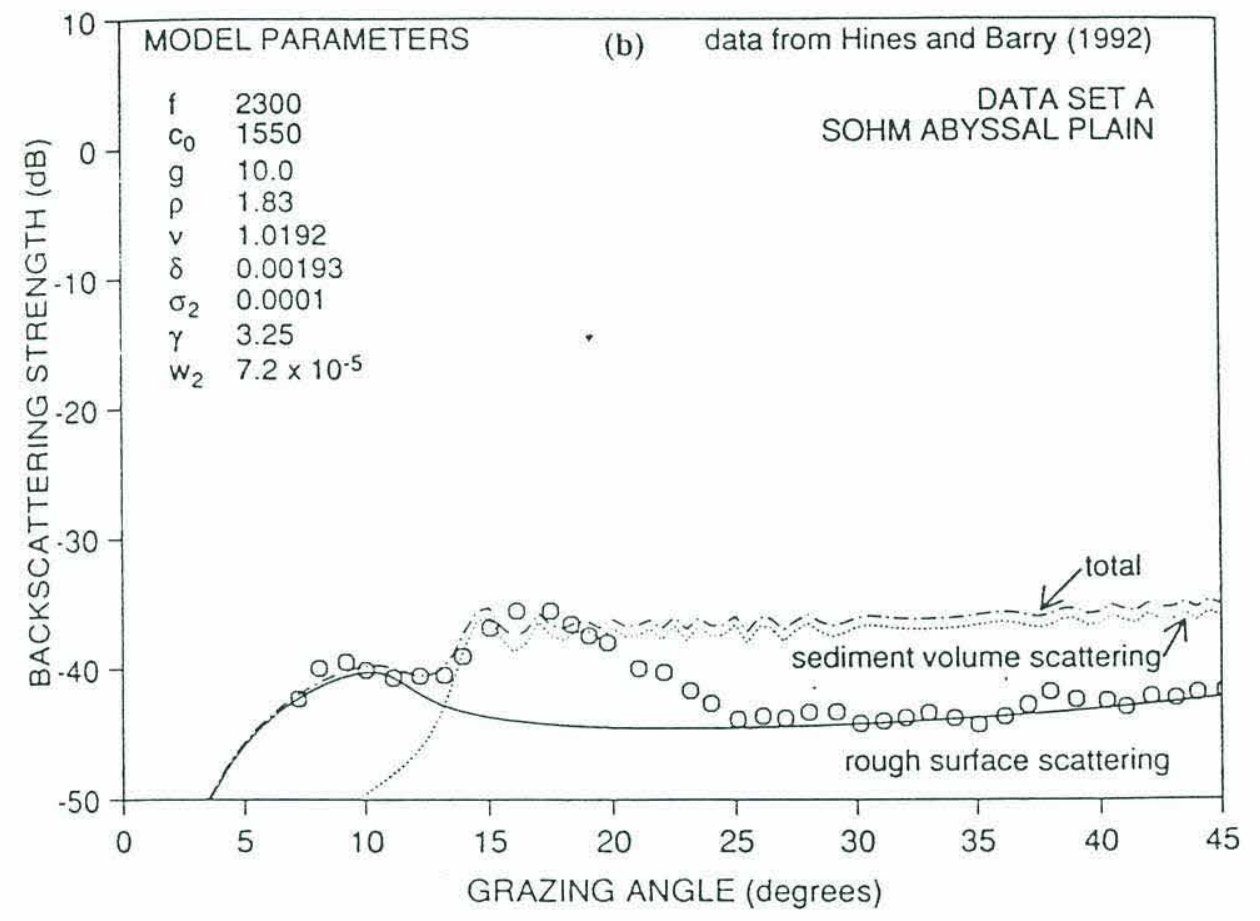

Figure 2-20: Figure 15(b) in Mourad and Jackson's paper [1]. The circles here represent the data points. 


\subsubsection{Three Layers with a Sound Speed Gradient in the Mid- dle Layer}

A more realistic model of the sediment is to have a basement beneath the water/sediment interface. We still choose a $1 / c^{2}$ linear sound speed profile below the water/sediment interface but constant sound velocity in the basement. Scatterers are assumed to be distributed in the middle layer. The presence of an additional layer interface enables the insonification of scatterers through some extra paths, i.e., the single or multiple bounces between the two interfaces, which is similar to some degree to the situation where there is a sound speed gradient in the bottom. The turning point there can be considered to be the virtual lower boundary.

One would gather that the middle layer thickness $H$ is likely to play an important role in the interference between multipath rays. Fig. 2-21 shows the field at the water/sediment interface for different middle layer thicknesses. The parameters for the middle layer are the same as those for Fig. 2-13(c) and correspond to a slow bottom. For the thin middle layer, the "frequency" of the oscillation is lower than that of the thick layer. However, the agreement between the far-field approximation and the exact solution is better. The "frequency" of the oscillation is related to the rate of the phase change for the transmitted wave with the change of the incident angle. For a thicker middle layer, a small change of the incident angle would cause a big change of the phase of the transmitted then reflected wave, which results in high "frequency" oscillations. The interference pattern will depend on the phase of the reflected rays and therefore on the layer thickness. The geoacoustic input for Fig. 2-22 for the upper two layers is the same as that for Fig. 2-12(b), corresponding to a sandy bottom. Although the argument for the "frequency" of the oscillation still holds, the level of oscillation is much smaller because of the high attenuation. In conclusion, the far-field approximation works better for a high-attenuation than for 

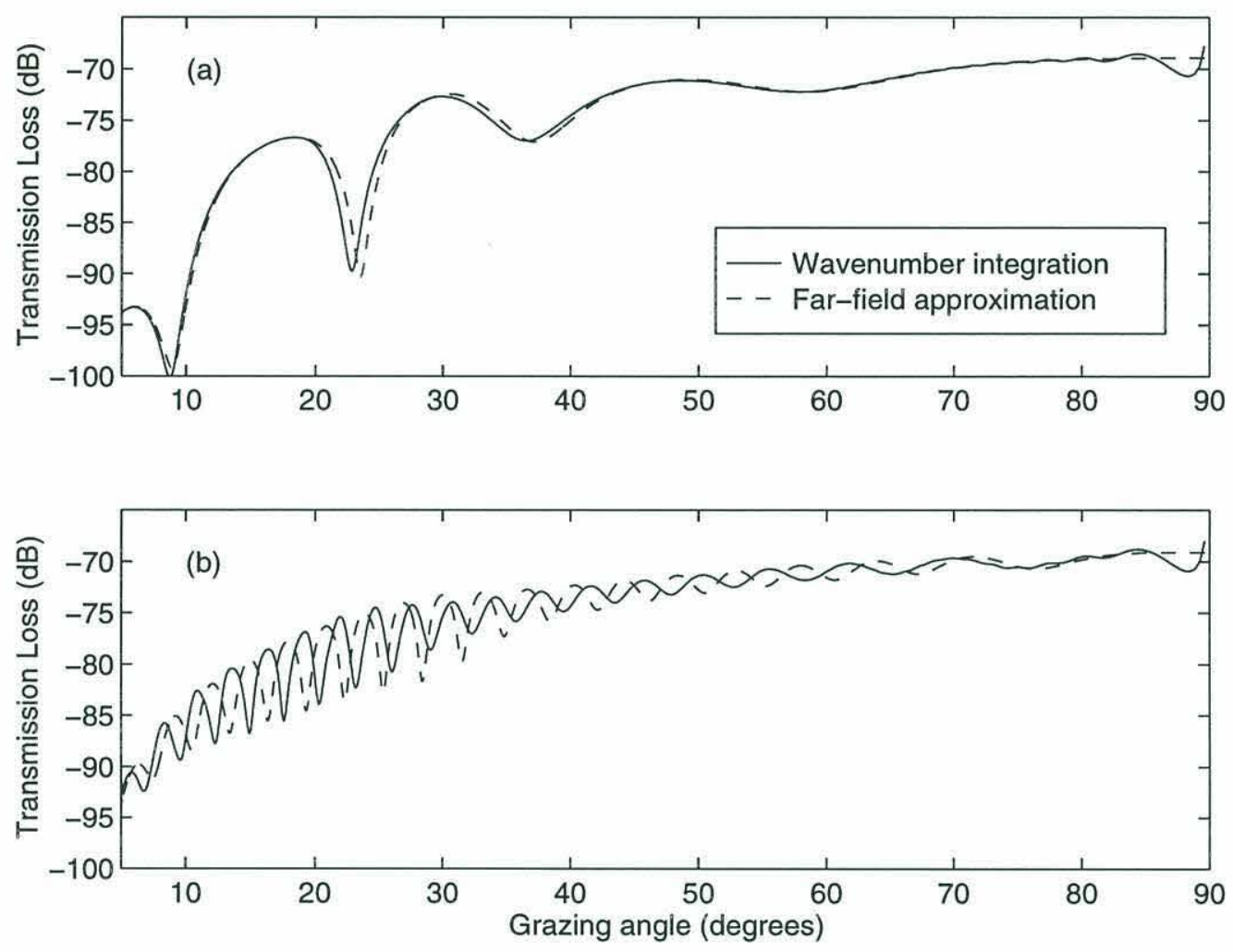

Figure 2-21: The middle layer thickness dependence for a three layer environment with a low attenuation middle layer. Parameters are: $f=500 \mathrm{~Hz}, c_{0}=1530 \mathrm{~m} / \mathrm{s}$, $c_{1}=1510 \mathrm{~m} / \mathrm{s}, c_{2}=1800 \mathrm{~m} / \mathrm{s}, \rho_{1}=1.72 \mathrm{~g} / \mathrm{cm}^{3}, \rho_{2}=1.9 \mathrm{~g} / \mathrm{cm}^{3}, \delta_{1}=0.00164$, $\delta_{2}=0.019, g=1 s^{-1}, z_{s}=100 \lambda, z=0$ and (a) $H=2 \lambda$; (b) $H=10 \lambda$. 

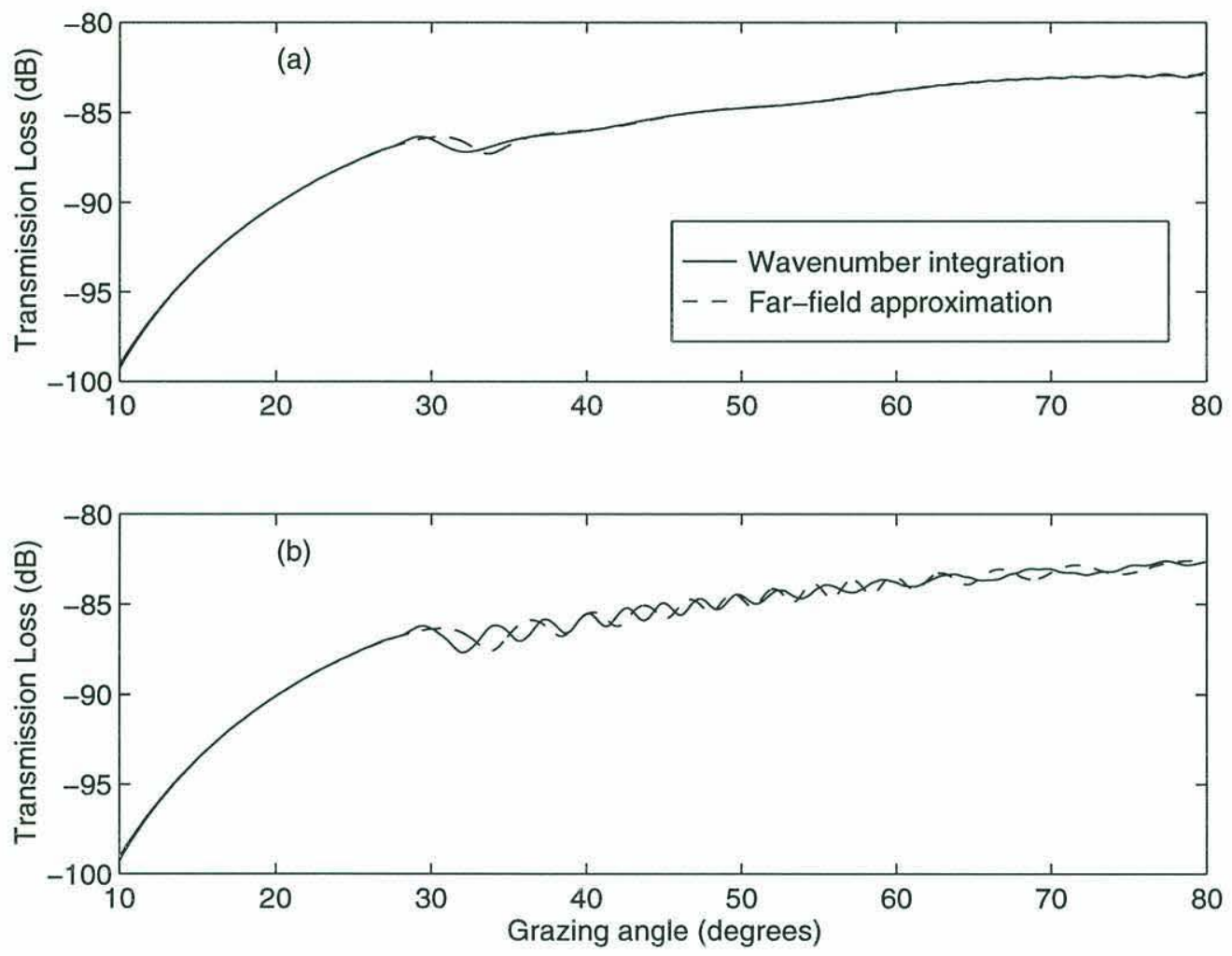

Figure 2-22: The middle layer thickness dependence for a three-layer environment with a high attenuation middle layer. Parameters are: $f=100 \mathrm{~Hz}, c_{0}=1500 \mathrm{~m} / \mathrm{s}$, $c_{1}=1689 \mathrm{~m} / \mathrm{s}, c_{2}=1800 \mathrm{~m} / \mathrm{s}, \rho_{1}=1.97 \mathrm{~g} / \mathrm{cm}^{3}, \rho_{2}=2.2 \mathrm{~g} / \mathrm{cm}^{3}, \delta_{1}=0.0166, \delta_{2}=$ $0.019, g=4 s^{-1}, z_{s}=100 \lambda, z=0$ and (a) $H=2 \lambda$; (b) $H=10 \lambda$. 
a low-attenuation bottom in three layer case.

\subsection{Summary}

In this chapter, we have compared several propagation models that are widely used in scattering studies with the exact solution obtained by a numerical wavenumber integration technique.

Hines' method, which decomposes the spherical wave into two plane waves: the refracted wave and the evanescent wave, matches the exact solution very well in a two isovelocity half-space scenario. The overestimate of the field in a small region close to the critical angle only affects the results at less than two degrees of grazing angle in our comparison, which is tolerable. Yet the difficulty in obtaining the stationary points easily and accurately hampers its generalization to more complex sound speed structure.

The far-field approximation, on the other hand, can be easily implemented in complicated bottom models, partly because it includes only one stationary point and has simplified its stationary point searching procedure by sacrificing some accuracy. As a result, the agreement with the exact solution is somewhat case-dependent. In a two isovelocity half-space model, the approach works almost perfectly if there doesn't exist the critical angle at the water/sediment interface, which is likely to be the case for a great amount of soft sediment types such as mud and silty clay. However, in some environments, the presence of the critical angle for such low-attenuation, soft sediment will find some underestimate of the field at subcritical angles. The situation is worse for deeper scatterers. This is because of the omission of the refracted wave past the critical angle inherent in the far-field approximation. The high attenuation in sandy bottoms would ease the problem to some extent by attenuating the refracted wave due to its long path. As for the influence on the equivalent surface scattering 
strength calculation, results are encouraging if the scattering layer starts from the water/sediment interface. However, when the scattering layer starts some distance away from the interface, which is unlikely for a sandy bottom but possible for soft sediments, the error introduced by applying the far-field approximation is significant.

The occurrence of the sound speed gradient in the sediment provides an additional propagation path, i.e., the turning refracted wave, which contributes to the determination of the interference pattern. The attenuation together with the frequency and the gradient controls the amplitude and phase of the turning refracted wave. Therefore, the results of comparisons are frequency- and gradient-dependent. In general, the far-field approximation fares better for a high-attenuation, low-gradient bottom. While for a low-attenuation bottom, the difference is considerably large, as can be seen in the equivalent surface scattering strength calculation.

Sediment layering is common in a real ocean environment. In the three layer case, the bounce of the acoustic wave between two interfaces adds to multipath phenomena. The oscillation of the field is sensitive to the layer thickness no matter what type of sediment is in the middle layer. High bottom attenuation is again helpful in alleviating the multipath effect, i.e., the oscillation. The far-field approximation matches the numerical wavenumber integration better for a thinner middle layer and a higher attenuation situation.

The validity of using the equivalent surface scattering strength to characterize the volume scattering process is debatable. Because of the evident multipath contribution to the field in many cases discussed in the comparison, the obtained angular dependence of the equivalent surface scattering strength is under question. However, as shown in our results, it is still reasonable to apply the equivalent surface scattering strength concept in some high-frequency and high-bottom attenuation bottom scattering studies. 


\section{Chapter 3}

\section{Formulations for Modeling Bottom Volume Backscattering}

A sound wave can be scattered by a region in which the acoustic properties of the medium differ from their values in the rest of the medium. Sound speed and density fluctuations are generally considered the major contributors to volume scattering. Although the effect of density fluctuation is insignificant within the water column, according to Chernov [33], it is often the dominant factor in the case of bottom backscattering from sediment volume inhomogeneities [14]. From a practical point of view, a statistical approach seems to be the only resort for people to model such small scale features. The fluctuations of sound speed and density in the bottom are usually small due to the nature of sedimentation process. Therefore, the method of small perturbation is suitable and widely used in volume scattering modeling [7, 14, 17, 28, 33, 35].

In this chapter we will derive the formula for modeling volume scattering due to sound speed and density fluctuations. It is similar to Morse and Ingard's [17], Chernov's [33] and Hines' [14] approaches and used by Tang in the study of shallow water reverberation [8]. Also, one section will concentrate on various power spectral density functions and correlation functions that are going to be used in describing sound speed and density fluctuations. In another section, we will present the analytical solution for backscattering from different kinds of random sound speed fluctuations 
in a free-space scenario.

\subsection{Volume Scattering Theory}

As shown in the previous chapter, the wave equation in an inhomogeneous medium can be written as

$$
\rho(\mathbf{r}) \nabla \cdot\left(\frac{1}{\rho(\mathbf{r})} \nabla p(\mathbf{r}, t)\right)-\frac{1}{c^{2}(\mathbf{r})} \frac{\partial^{2} p(\mathbf{r}, t)}{\partial t^{2}}=f(\mathbf{r}, t)
$$

where $p(\mathbf{r}, t)$ represents the pressure, $\rho(\mathbf{r})$ represents the density, $c(\mathbf{r})$ represents the sound speed, and $f(\mathbf{r}, t)$ represents the source term as a function of space $\mathbf{r}$ and time $t$. Assuming a point source and harmonic time dependence $\exp (i \omega t)$, the inhomogeneous Helmholtz equation is [33]:

$$
\left[\nabla^{2}+k^{2}(\mathbf{r})\right] P(\mathbf{r})-\frac{\nabla \rho(\mathbf{r})}{\rho(\mathbf{r})} \cdot \nabla P(\mathbf{r})=S_{\omega} \delta\left(\mathbf{r}-\mathbf{r}_{\mathbf{s}}\right),
$$

where the source strength is $S_{\omega}$. We assume that the background density is constant and the background sound speed only varies with depth $z$. With small variations in both density and sound speed, we have

$$
\begin{aligned}
& \rho(\mathbf{r})=\rho_{0}+\delta \rho(\mathbf{r}) \\
& c(\mathbf{r})=c_{0}(z)+\delta c(\mathbf{r}),
\end{aligned}
$$

where $\delta \rho(\mathbf{r}) \ll \rho_{0}$ and $\delta c(\mathbf{r}) \ll c_{0}(z)$ and they both have zero means.

If we expand the Helmholtz equation and keep up to first-order of smallness with respect to $\rho$ and $c$, Eq.3.2 becomes

$$
\left[\nabla^{2}+k_{0}^{2}(z)-\frac{2 \delta c(\mathbf{r}) k_{0}^{2}(z)}{c_{0}(z)}\right] P(\mathbf{r})-\frac{\nabla(\delta \rho(\mathbf{r}))}{\rho_{0}} \cdot \nabla P(\mathbf{r})=S_{\omega} \delta\left(\mathbf{r}-\mathbf{r}_{\mathbf{s}}\right)
$$


The total pressure field can also be expressed as the summation of the mean field $P_{0}(\mathbf{r})$ and the scattered field $P_{s}(\mathbf{r})$ :

$$
P(\mathbf{r})=P_{0}(\mathbf{r})+P_{s}(\mathbf{r})
$$

We can obtain the equation for the mean field $P_{0}(\mathbf{r})$ by inserting this summation in the equation above and averaging:

$$
\begin{aligned}
& {\left[\nabla^{2}+k_{0}^{2}(z)\right] P_{0}(\mathbf{r})-\left\langle\frac{2 \delta c(\mathbf{r}) k_{0}^{2}(z)}{c_{0}(z)} P_{s}(\mathbf{r})\right\rangle} \\
& -\left\langle\frac{\nabla(\delta \rho(\mathbf{r}))}{\rho_{0}} \cdot \nabla P_{s}(\mathbf{r})\right\rangle=S_{\omega} \delta\left(\mathbf{r}-\mathbf{r}_{\mathbf{s}}\right) .
\end{aligned}
$$

Subtracting this equation from the full averaged Helmholtz equation, we can get an equation for the scattered field:

$$
\left[\nabla^{2}+k_{0}^{2}(z)\right] P_{s}(\mathbf{r})=\frac{2 \delta c(\mathbf{r}) k_{0}^{2}(z)}{c_{0}(z)} P_{0}(\mathbf{r})+\frac{\nabla(\delta \rho(\mathbf{r}))}{\rho_{0}} \cdot \nabla P_{0}(\mathbf{r})
$$

It is interesting to notice that the two terms on the RHS caused by sound speed and density fluctuation act like virtual sources. As discussed by Morse and Ingard [17], the first term comes from the fact that the small region at $\mathbf{r}$ doesn't have the same sound speed (therefore compressibility) as the surrounding medium, which results in a monopole-like source distribution. The second term gives rise to dipole-like scattering phenomenon because the small region doesn't move in response to the force $\nabla P_{0}$ with the same velocity as does the surrounding medium due to the difference in density. This dipole source would have a null in the forward scattering direction but its major axis is in the backscattering direction, which makes density fluctuations a significant contributor to backscattering. As a result, it is of great importance to include density variations in volume backscattering modeling.

For the weak volume scattering problem, a usual assumption is that the effect of the scattered field on the mean field can be ignored (Born Approximation). Therefore, 
Eq.3.6 becomes:

$$
\left[\nabla^{2}+k_{0}^{2}(z)\right] P_{0}(\mathbf{r})=S_{\omega} \delta\left(\mathbf{r}-\mathbf{r}_{\mathbf{s}}\right)
$$

The corresponding Green's function can be obtained from

$$
\left[\nabla^{2}+k_{0}^{2}(\mathbf{r})\right] G\left(\mathbf{r}, \mathbf{r}_{\mathbf{s}}\right)=\delta\left(\mathbf{r}-\mathbf{r}_{\mathbf{s}}\right)
$$

and the boundary conditions.

Also, the sound speed variation and density variation are often considered linked, which would simplify the problem. As discussed by Hines [14], for a random sediment bottom, both fluctuations can be expressed as functions of the bottom porosity $p$ :

$$
\begin{aligned}
& \delta c=\frac{d c}{d p} \delta p \\
& \delta \rho=\frac{d \rho}{d p} \delta p
\end{aligned}
$$

where $\delta p$ is the variation in bottom porosity. If we use the notation

$$
\begin{aligned}
\epsilon(\mathbf{r}) & \equiv \frac{\delta c}{c_{0}} \\
\frac{\delta \rho}{\rho_{0}} & \equiv \beta \epsilon(\mathbf{r}),
\end{aligned}
$$

where $\beta$ is the ratio between density variation and sound speed variation, the scattered field equation becomes

$$
\left[\nabla^{2}+k_{0}^{2}(z)\right] P_{s}(\mathbf{r})=2 k_{0}^{2}(z) \epsilon(\mathbf{r}) P_{0}(\mathbf{r})+\beta \nabla \epsilon(\mathbf{r}) \cdot \nabla P_{0}(\mathbf{r}) .
$$

Applying Green's Theorem to the equation above, we have

$$
P_{s}\left(\mathbf{R}_{\mathbf{r}}\right)=\int_{v}\left[2 k_{0}^{2}\left(z^{\prime}\right) \epsilon\left(\mathbf{r}^{\prime}\right) P_{0}\left(\mathbf{r}^{\prime}\right)+\beta \nabla \epsilon\left(\mathbf{r}^{\prime}\right) \cdot \nabla P_{0}\left(\mathbf{r}^{\prime}\right)\right] G\left(\mathbf{R}_{\mathbf{r}}, \mathbf{r}^{\prime}\right) d \mathbf{r}^{\prime}
$$


where $\mathbf{R}_{\mathbf{r}}$ stands for the receiver position and $\mathbf{r}^{\prime}$ stands for the scatterer position. After integration by parts, the scattered field equation can be further expressed as

$$
P_{s}\left(\mathbf{R}_{\mathbf{r}}\right)=\int_{v} \epsilon\left(\mathbf{r}^{\prime}\right)\left[(2+\beta) k_{0}^{2}\left(z^{\prime}\right) P_{0}\left(\mathbf{r}^{\prime}\right) G\left(\mathbf{R}_{\mathbf{r}}, \mathbf{r}^{\prime}\right)-\beta\left(\nabla P_{0}\left(\mathbf{r}^{\prime}\right) \cdot \nabla G\left(\mathbf{R}_{\mathbf{r}}, \mathbf{r}^{\prime}\right)\right)\right] d \mathbf{r}^{\prime}
$$

The scattered intensity $I_{s}$ can then be obtained by

$$
\begin{aligned}
I_{s}= & \left\langle\left|P_{s}\left(\mathbf{R}_{\mathbf{r}}\right)\right|^{2}\right\rangle \\
= & \int_{v_{1}} \int_{v_{2}}\left\langle\epsilon\left(\mathbf{r}_{\mathbf{1}}\right) \epsilon^{*}\left(\mathbf{r}_{\mathbf{2}}\right)\right\rangle\left[(2+\beta) k_{0}^{2}\left(z_{1}\right) P_{0}\left(\mathbf{r}_{\mathbf{1}}\right) G\left(\mathbf{R}_{\mathbf{r}}, \mathbf{r}_{\mathbf{1}}\right)-\beta\left(\nabla P_{0}\left(\mathbf{r}_{\mathbf{1}}\right) \cdot \nabla G\left(\mathbf{R}_{\mathbf{r}}, \mathbf{r}_{\mathbf{1}}\right)\right)\right] \\
& {\left[(2+\beta) k_{0}^{2}\left(z_{2}\right) P_{0}^{*}\left(\mathbf{r}_{\mathbf{2}}\right) G^{*}\left(\mathbf{R}_{\mathbf{r}}, \mathbf{r}_{\mathbf{2}}\right)-\beta\left(\nabla P_{0}^{*}\left(\mathbf{r}_{\mathbf{2}}\right) \cdot \nabla G^{*}\left(\mathbf{R}_{\mathbf{r}}, \mathbf{r}_{\mathbf{2}}\right)\right)\right] d \mathbf{r}_{\mathbf{1}} d \mathbf{r}_{\mathbf{2}} . }
\end{aligned}
$$

Here $\left(^{*}\right)$ stands for complex conjugate operation.

In the above equation, $\left\langle\epsilon\left(\mathbf{r}_{1}\right) \epsilon^{*}\left(\mathbf{r}_{2}\right)\right\rangle$ is the correlation function of the sound speed fluctuation. Since the value of the sound speed variation must be a real number, $\left({ }^{*}\right)$ can be omitted from the correlation function expression. In this weak volume scattering model with the Born approximation, we can see that the scattered intensity is determined by the second-order statistics of the random sound speed and density variations if the propagation part is known. So it is worthwhile to devote the next section to different kinds of correlation functions to be used in volume scattering modeling.

If scattering time series are of interest, we can apply the technique of Fourier synthesis. The scattered return $y(t)$ can be calculated by

$$
y(t)=\frac{1}{2 \pi} \int_{-\infty}^{\infty} P_{s}\left(\mathbf{R}_{\mathbf{r}}, \omega\right) S(\omega) \exp (i \omega t) d \omega
$$

where $\omega$ is the radial frequency and $S(\omega)$ is the spectrum of the source signal. This equation will be used in Chapter 5 to generate scattering time series.

The validity of the volume scattering formula is not restricted to layered fluid 
media. As far as scatterers are located in the fluid layer, the bottom can be fluid, fluid-elastic, or elastic layered media. The random scattering layer is not necessarily immediately beneath the water/sediment interface. The attenuation of both compressional waves and shear waves and the background sound speed gradient are easily incorporated into the model because they are taken care of in the calculation of the Green's function by OASES. This theory will be suitable for studying scattering by a sediment bottom, where interface roughness does not necessarily dominate the scattering process. This is exactly the situation that we encountered in the ARSRP scattering experiment.

\subsection{Correlation Functions of Sound Speed Varia- tions}

The second-order statistics of sound speed and density variations are of great importance to volume scattering modeling, as can be seen in the section above. However, it is difficult to have direct measurement of such sediment properties, let alone a good model of them. Traditionally, Gaussian and exponential correlation functions have often been used because of their mathematical simplicity [14, 33, 35]. Recently, a power-law type of correlation function has attracted more interest [53, 63]. Meanwhile, Yamamoto, in his measurement of velocity variability using crosswell acoustic tomography, found the measured power spectra approximate a power law [64]. Still it is hardly conclusive that the power-law type power spectral density is the best description of velocity variations in the sediment. Therefore, we choose to include all three of them in our model.

In the beginning of this chapter, we have defined two random variables, the relative sound speed variation $\epsilon$ and the density variation. Since they are assumed to be fully correlated, only the statistics of $\epsilon$ will be dealt with from now on. In addition to the 
zero mean assumption regarding $\epsilon$, we require that the sound speed fluctuation be a homogeneous random variable. In other words, the process is spatially wide-sense stationary, by which we mean that the covariance of the random variable is invariant with respect to spatial translation,

$$
\left\langle\epsilon\left(\mathbf{r}_{1}\right) \epsilon\left(\mathbf{r}_{2}\right)\right\rangle=C\left(\mathbf{r}_{1}-\mathbf{r}_{2}\right) .
$$

One may argue that stationarity can never be achieved in a real environment. Nevertheless, from a practical point of view, it is a reasonable assumption if we confine ourselves to a small enough region so that the change across the whole region is little. Alternatively, a random process can be characterized in the wavenumber domain by its power spectrum

$$
W(\mathbf{k})=\int_{-\infty}^{\infty} \int_{-\infty}^{\infty} \int_{-\infty}^{\infty} C(\mathbf{r}) \exp (-i \mathbf{k} \cdot \mathbf{r}) d \mathbf{r}
$$

where $\mathbf{k}=\left(k_{x}, k_{y}, k_{z}\right)$ is the wavenumber vector.

For a horizontal correlation function to be Gaussian or exponential, we assume that the correlation function of the quantity $\epsilon(\mathbf{r})$ is horizontally and vertically decoupled $[7,28,35]$ :

$$
C\left(\mathbf{r}_{1}-\mathbf{r}_{2}\right)=\left\langle\epsilon\left(\mathbf{r}_{1}\right) \epsilon\left(\mathbf{r}_{2}\right)\right\rangle=\sigma^{2} N(\mathbf{l}) M\left(\left|z_{1}-z_{2}\right|\right),
$$

where the horizontal vector $\mathbf{l}=\left[\left(x_{1}-x_{2}\right),\left(y_{1}-y_{2}\right)\right]$, and $\sigma$ is the standard deviation of $\epsilon$. Geoacoustic evidence [29] showed that the vertical correlation length $l_{z}$ is much smaller than the horizontal correlation length $l_{x}$ and $l_{y}$. For the sake of convenience, the vertical correlation function $M(z)$ is chosen to be exponential, i.e.,

$$
M(z)=\exp \left(-\frac{|z|}{l_{z}}\right)
$$


Its Fourier transform is

$$
\tilde{W}\left(k_{z}\right)=\frac{2 l_{z}}{1+\left(k_{z} l_{z}\right)^{2}} .
$$

The horizontal correlation function $N(\mathbf{l})$ can be both Gaussian and exponential. For a Gaussian correlation function, we have

$$
N(x, y)=\exp \left(-\frac{1}{2}\left[\left(\frac{x}{l_{x}}\right)^{2}+\left(\frac{y}{l_{y}}\right)^{2}\right]\right) .
$$

(Notice a factor of $1 / 2$ difference in the exponential compared to that in Chernov's [33] and Tang's [35] work.) The corresponding Fourier transform is

$$
\tilde{W}\left(k_{x}, k_{y}\right)=2 \pi l_{x} l_{y} \exp \left(-\frac{k_{x}^{2} l_{x}^{2}+k_{y}^{2} l_{y}^{2}}{2}\right) .
$$

As for the exponential correlation function, there are two choices: one is for an isotropic random field,

$$
N(\mathbf{l})=\exp \left(-\frac{|\mathbf{l}|}{l_{0}}\right),
$$

with $l_{0}$ the correlation length and the Fourier transform

$$
\tilde{W}\left(k_{r}\right)=\frac{2 \pi l_{0}^{2}}{\left[1+\left(k_{r} l_{0}\right)^{2}\right]^{3 / 2}} ;
$$

the other is for an anisotropic random field

$$
N(x, y)=\exp \left(-\left[\frac{|x|}{l_{x}}+\frac{|y|}{l_{y}}\right]\right),
$$

with a corresponding Fourier transform

$$
\tilde{W}\left(k_{x}, k_{y}\right)=\frac{4 l_{x} l_{y}}{\left(1+k_{x}^{2} l_{x}^{2}\right)\left(1+k_{y}^{2} l_{y}^{2}\right)} .
$$

The second function will not degenerate to the first one even if $l_{x}=l_{y}$, because 
of a discontinuity at zero lag. However, as discussed by Ivakin and Lysanov [28], this can be corrected by introducing a second length scale to represent the minimum inhomogeneity scale in Eq.3.24, thereby obtaining

$$
N(\mathbf{l})=\frac{\exp \left(-\frac{|1|}{l_{0}}\right)-\varepsilon \exp \left(-\frac{|1|}{l_{1}}\right)}{1-\varepsilon},
$$

where $\varepsilon=l_{1} / l_{0}$ and $l_{1} \ll l_{0}$. Under the condition that $\varepsilon \rightarrow 0$, it is equivalent to Eq.3.24.

The Von Karman function, which has a power-law type of spectrum, is used as a correlation function to describe turbulence (Tatarski [65]), to characterize heterogeneity in the earth's crust (Frankel and Clayton [63]), and to model the seafloor morphology (Goff and Jordan [66]). In this work, we will follow Goff and Jordan's approach. First, a set of functions are defined

$$
G_{\nu}(b(\mathbf{r}))=b^{\nu}(\mathbf{r}) K_{\nu}(b(\mathbf{r})) \quad 0 \leq b<\infty,
$$

where $K_{\nu}$ is the modified Bessel function of order $\nu$ and

$$
b(\mathbf{r})=\left[\mathbf{r}^{T} Q \mathbf{r}\right]^{1 / 2} .
$$

Here $(T)$ stands for the transpose operation, and $Q$ is a positive-definite, symmetric matrix whose Cartesian elements $q_{i j}$ have dimensions of $(\text { length })^{-2}$. In the threedimensional case, it can be expressed in terms of its normalized eigenvectors $\mathbf{e}_{\mathbf{n}}, \mathbf{e}_{\mathbf{s}}$, and $\mathbf{e}_{\mathbf{t}}$ and its ordered eigenvalues $k_{n}^{2} \geq k_{s}^{2} \geq k_{t}^{2}$,

$$
Q=k_{n}^{2} \mathbf{e}_{\mathbf{n}} \mathbf{e}_{\mathrm{n}}^{\mathrm{T}}+k_{s}^{2} \mathbf{e}_{\mathbf{s}} \mathbf{e}_{\mathbf{s}}^{\mathrm{T}}+k_{t}^{2} \mathbf{e}_{\mathbf{t}} \mathbf{e}_{\mathbf{t}}^{\mathrm{T}} .
$$

The $Q$ matrix carries information about the anisotropy of the random field. While $k_{n}, k_{s}$ and $k_{t}$ correspond to the inverses of the correlation lengths in three dimensions respectively. For a two-dimensional case, simply drop the $k_{t}$ term in the expression. 
The correlation function can then be set to be

$$
C(\mathbf{r})=\sigma^{2} \frac{G_{\nu}(b(\mathbf{r}))}{G_{\nu}(0)} .
$$

The power spectrum for a two-dimensional random field is

$$
W\left(k_{x}, k_{y}\right)=\left\{\begin{array}{l}
\frac{4 \pi \nu \sigma^{2} l_{x} l_{y}}{\left(1+k_{x}^{2} l_{x}^{2}+k_{y}^{2} l_{y}^{2}\right)^{\nu+1}} \nu \neq 0 . \\
\frac{4 \pi \sigma^{2} l_{x} l_{y}}{\left(1+k_{x}^{2} l_{x}^{2}+k_{y}^{2} l_{y}^{2}\right)} \nu=0
\end{array}\right.
$$

Because we are interested in monostatic backscattering in this work, azimuthallysummed random fields will be generated as shown in Chapter 4 . The $x$ and $y$ axis are then chosen to be overlaid with $\mathbf{e}_{\mathbf{n}}$ and $\mathbf{e}_{\mathbf{s}}$, i.e., no skew. Figures 3-1 and 3-2 show the correlation functions and power spectra for several value of $\nu$ (same as Fig. 2 in Ref. [66]). An asymptotic roll-off rate $-2(\nu+1)$ of the spectrum is related to the fractal(Hausdorff) dimension $D_{\text {fractal }}$ of such a stochastic process as:

$$
D_{\text {fractal }}=3-\nu \text {. }
$$

Therefore, decreasing the parameter $\nu$ would increase the roughness, which is the case for the three-dimensional case as well. In the special case of $\nu=1 / 2$, the power spectrum reduces to the isotropic exponential power spectrum when $l_{x}=l_{y}$. Of course, we can construct a vertically uncorrelated random field model with the randomness in each layer specified by the above two-dimensional power-law type of power spectrum. For a correlated three-dimensional case, the power spectrum is

$$
W\left(k_{x}, k_{y}, k_{z}\right)=\frac{8 \pi \sqrt{\pi} l_{x} l_{y} l_{z} \sigma^{2} \Gamma(\nu+3 / 2)}{\Gamma(\nu)\left(1+l_{x}^{2} l_{x}^{2}+k_{y}^{2} l_{y}^{2}+k_{z}^{2} l_{z}^{2}\right)^{\nu+3 / 2}},
$$

where $\Gamma$ represents the Gamma function. The roll-off rate now is $-2(\nu+3 / 2)$ and the related fractal(Hausdorff) dimension is

$$
D_{\text {fractal }}=4-\nu \text {. }
$$




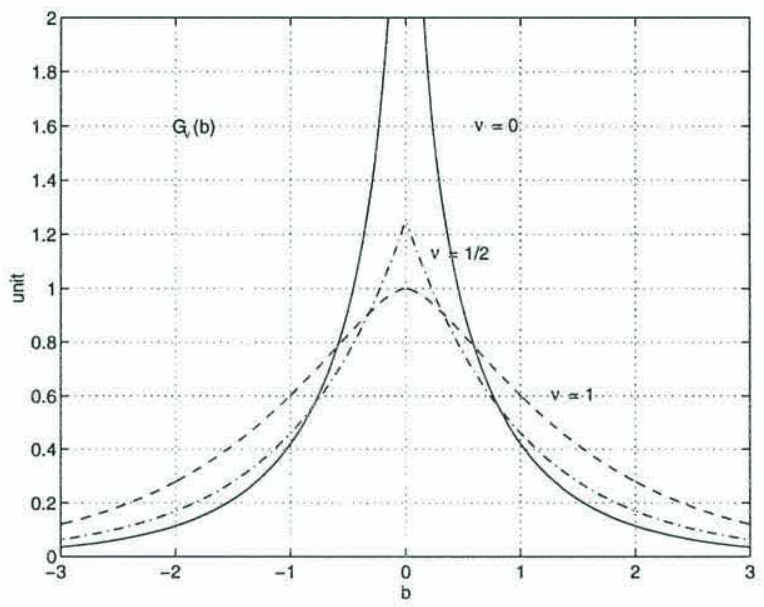

Figure 3-1: Functional form of the correlation function $G_{\nu}(b)$ plotted for values of $\nu=0,1 / 2$, and 1 .

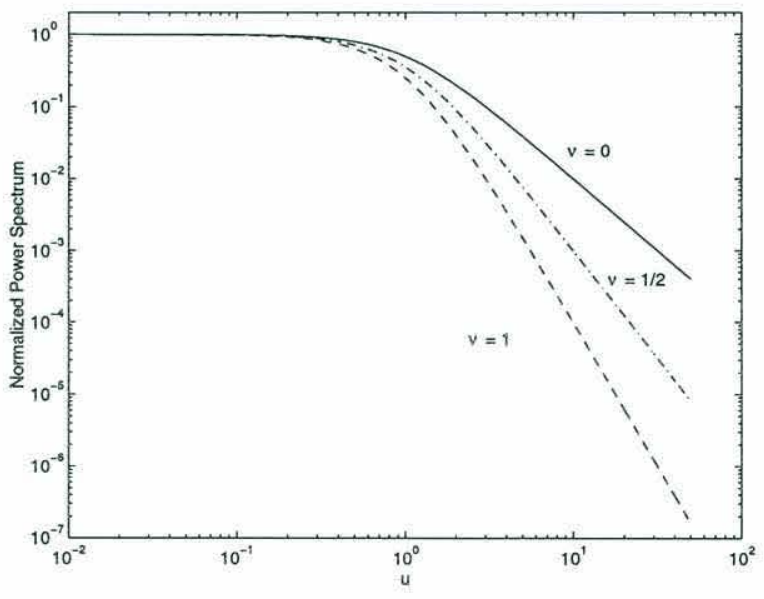

Figure 3-2: Normalized power spectra plotted in log-log space. 


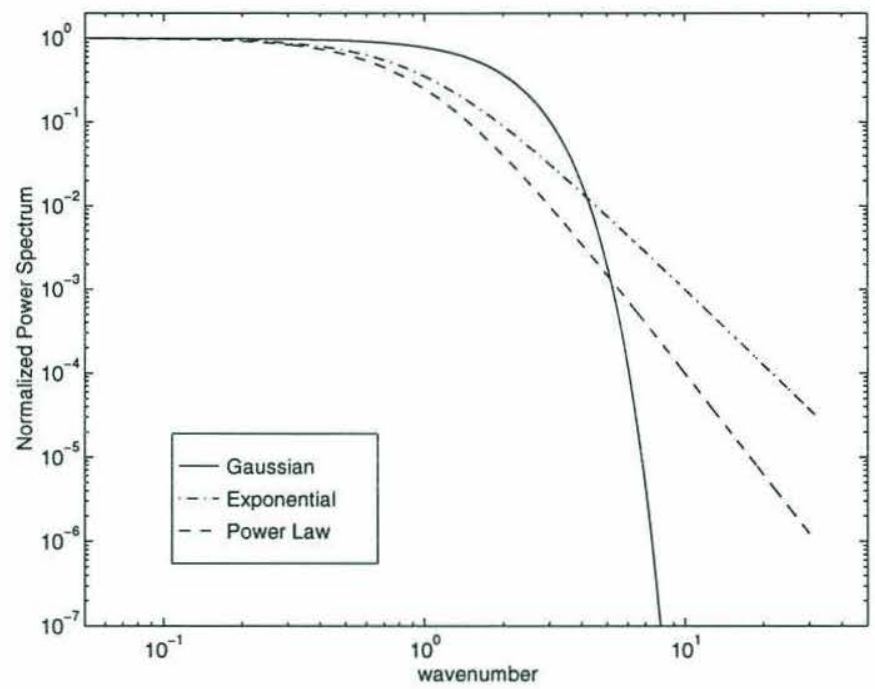

Figure 3-3: Normalized isotropic power spectra for different kinds of correlation functions $\left(l_{x}=l_{y}=1 m, \nu=1\right.$ in power law distribution).

As can be seen in Fig. 3-3, the isotropic power spectra $\left(l_{x}=l_{y}\right)$ for Gaussian, exponential and power law distributions have different roll-off rates versus wavenumber even with the same correlation length. For a Gaussian distribution, the spectral level at high wavenumber is low, which translates to a lack of small-scale roughness. On the other hand, there are significant amounts of energy in high wavenumbers for exponential and power law distributions. This is consistent with the fact that there is abundant small-scale roughness with the latter two distributions.

\subsection{Backscattering from Sound Speed Variations in the Free-Space Scenario: Analytical Solu- tions}

Analytical solutions for volume backscattering can be achieved in some special situations such as the free-space scenario. By free space, we mean that the background 


\section{Free-Space Scenario}

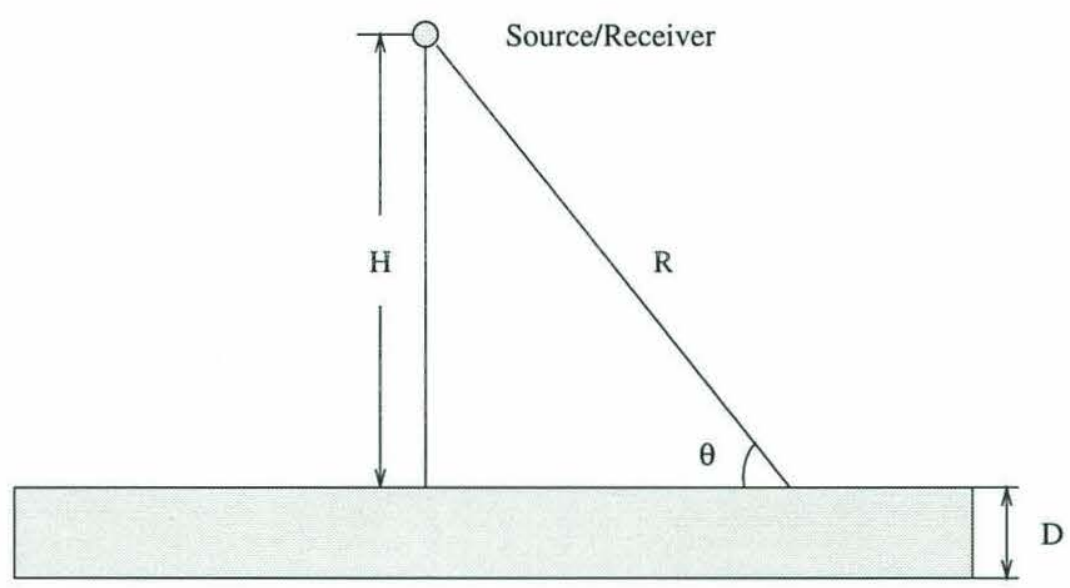

Figure 3-4: The free-space monostatic backscattering scenario.

sound speed and density are constants throughout the media. Because monostatic backscattering is the concentration of this work, solutions will be given only for this scenario. Meanwhile, only sound speed variations will be discussed for the sake of mathematical simplicity, i.e., $\beta=0$ (the effect of density variations will be studied later in Chapter 5). Figure 3-4 shows the scenario that we are going to study in this section. In the figure, $H$ represents source/receiver height, $\theta$ is the scattering angle, $D$ is the thickness of the random scattering layer, and $R$ is the distance between source/receiver and scatterers. We choose the background sound speed to be $1500 \mathrm{~m} / \mathrm{s}$ and the density to be $1000 \mathrm{~kg} / \mathrm{m}^{3}$ for the rest of the chapter.

In free space, the Green's function for a point source is well known as:

$$
G\left(\mathbf{R}_{\mathbf{r}}, \mathbf{r}\right)=-\frac{\exp (-i \mathbf{k} \cdot \mathbf{R})}{4 \pi R}
$$

where $\mathbf{R}=\mathbf{R}_{\mathbf{r}}-\mathbf{r}$ and $R=|\mathbf{R}|$. Therefore, for monostatic backscattering and unit 
source strength, Eq.3.15 becomes

$$
I_{s}=4 k^{4} \int_{v_{1}} \int_{v_{2}} C\left(\mathbf{R}_{\mathbf{1}}-\mathbf{R}_{\mathbf{2}}\right) \frac{\exp \left(-2 i \mathbf{k} \cdot\left(\mathbf{R}_{\mathbf{1}}-\mathbf{R}_{\mathbf{2}}\right)\right)}{\left(4 \pi R_{1}\right)^{2}\left(4 \pi R_{2}\right)^{2}} d \mathbf{r}_{\mathbf{1}} d \mathbf{r}_{\mathbf{2}} .
$$

If we have $R_{1}$ and $R_{2}$ much larger than the scattering volume dimension $L$, with $L$ much larger than the correlation length of the random field, $R_{1}$ and $R_{2}$ can be approximated by the distance between the source/receiver and a reference point in the scattering volume $R$. After performing the coordinate transformation to center coordinate $\mathbf{r}_{\mathbf{0}}$ and relative coordinate $\xi$, we have

$$
I_{s}=\frac{4 k^{4}}{(4 \pi R)^{4}} \int_{v} \int_{-\infty}^{\infty} C(\xi) \exp (-2 i \mathbf{k} \cdot \xi) d \xi d \mathbf{r}_{0} .
$$

Bear in mind that the correlation function depends only on the relative coordinate. By expanding the integration limit of the relative coordinate to infinity, we have actually neglected the influence due to the existence of physical boundaries, which will result in some differences when compared with solutions including the boundary effect. For a thin layer of scatterers, the equivalent surface backscattering coefficient $\Sigma$ can be obtained by

$$
\Sigma=\frac{I_{s} R^{2}}{I_{\text {in }} A},
$$

where $I_{i n}$ is the intensity of the incident source wave a unit distance away from the scattering region, $R$ is defined above as the distance between the source/receiver and a reference point in the scattering region, and $A$ is the insonified area(here actually the top of the scattering volume) [52]. The backscattering strength is simply

$$
B S S=10 \log _{10} \Sigma .
$$

For a point source with unit source strength,

$$
I_{\text {in }}=\frac{1}{(4 \pi R)^{2}}
$$


Therefore, $\Sigma$ can be calculated by

$$
\Sigma=\frac{k^{4}}{4 \pi^{2} A} \int_{v} \int_{v} C(\xi) \exp (-2 i \mathbf{k} \cdot \xi) d \xi d \mathbf{r}_{0}
$$

In the rest of the section, we will compute $\Sigma$ for different correlation functions that have been introduced in the previous section. Although the goal of this work is to model monostatic backscattering from the inhomogeneities of a full three-dimensional random field, we opt to investigate first backscattering from two-dimensional pseudosurfaces with sound speed variations (instead of height perturbations in the usual surface scattering problem) in hopes of obtaining some insights before complicating the problem further with another dimension. In fact, scattering from this pseudosurface is equivalent to that from a vertically uncorrelated $1 \mathrm{~m}$ layer with the same horizontal correlation structure. While at the end of this section, the effect of vertical correlation will be evaluated.

\subsubsection{Backscattering from 2-D Isotropic Random Field}

By isotropic we mean that the correlation lengths for the random field in $x$ and $y$ direction $l_{x}$ and $l_{y}$ are equal to $l_{r}$.

For a Gaussian correlation function, the backscattering coefficient can be obtained as:

$$
\Sigma(\theta)=\frac{k^{4} \sigma^{2} l_{r}^{2}}{2 \pi} \exp \left(-2 k^{2} l_{r}^{2} \cos ^{2} \theta\right)
$$

where $\theta$ is the scattering grazing angle.

Figure 3-5 shows the relation between the backscattering strength and the product of horizontal wavenumber $K$ and $a$, which equals to $\sqrt{2} l_{r}$ for the Gaussian distribution. It can be noticed that the backscattering strength has a peak around $K a=1$ and decays rapidly otherwise. As discussed by Chernov [33], $K a \ll 1$ corresponds 


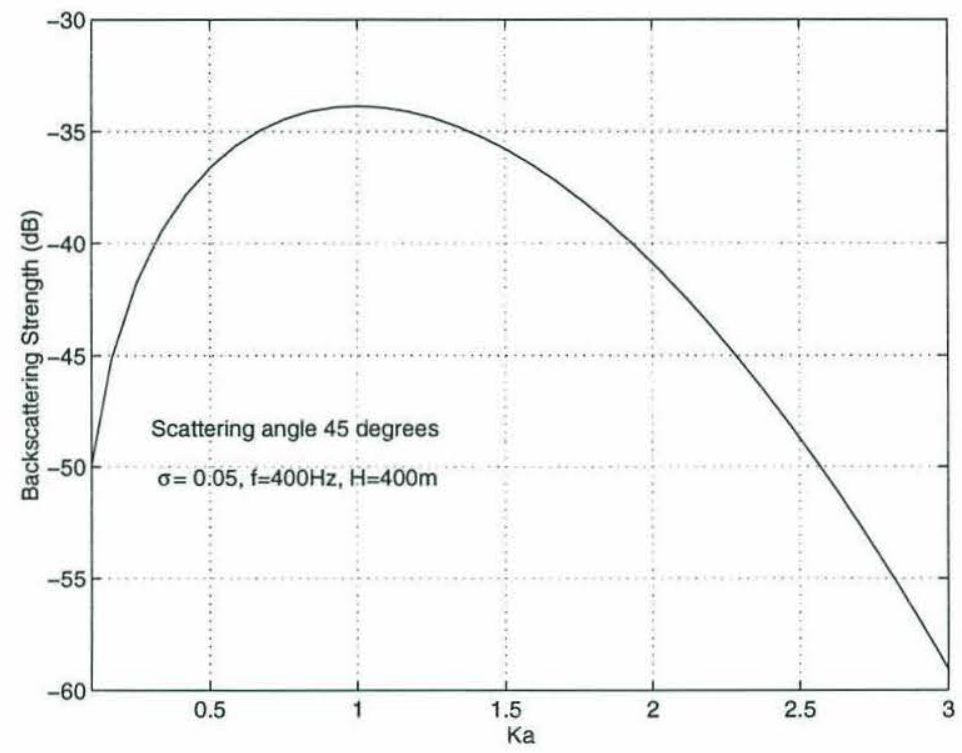

Figure 3-5: Backscattering from sound speed variations with a 2-D isotropic Gaussian correlation function.

to backscattering from small-scale inhomogeneities, which results in isotropic scattering. For $K a \gg 1$, the scattered power will be concentrated at a small forward angle; therefore the backscattering is weakened. The reason that the backscattering has only one "resonance" for the Gaussian distribution has a lot to do with the fact that the Gaussian distribution is a single-scale model.

As for an isotropic exponential correlation function, the backscattering coefficient is

$$
\Sigma(\theta)=\frac{k^{4} \sigma^{2} l_{r}^{2}}{2 \pi\left[1+\left(2 k l_{r} \cos \theta\right)^{2}\right]^{3 / 2}}
$$

As shown in Fig. 3-6, the backscattering strength decays much slower versus $K a$ than with a Gaussian distribution. Here $K$ again is the horizontal wavenumber and $a=l_{r}$. The explanation for this behavior is that a random field with an exponential correlation function has its energy distributed almost equally among all wavenumbers, i.e., it is a multi-scale model. It means that the random field would have roughness at all the scales, which makes it an effective scatterer for various wavelengths or 


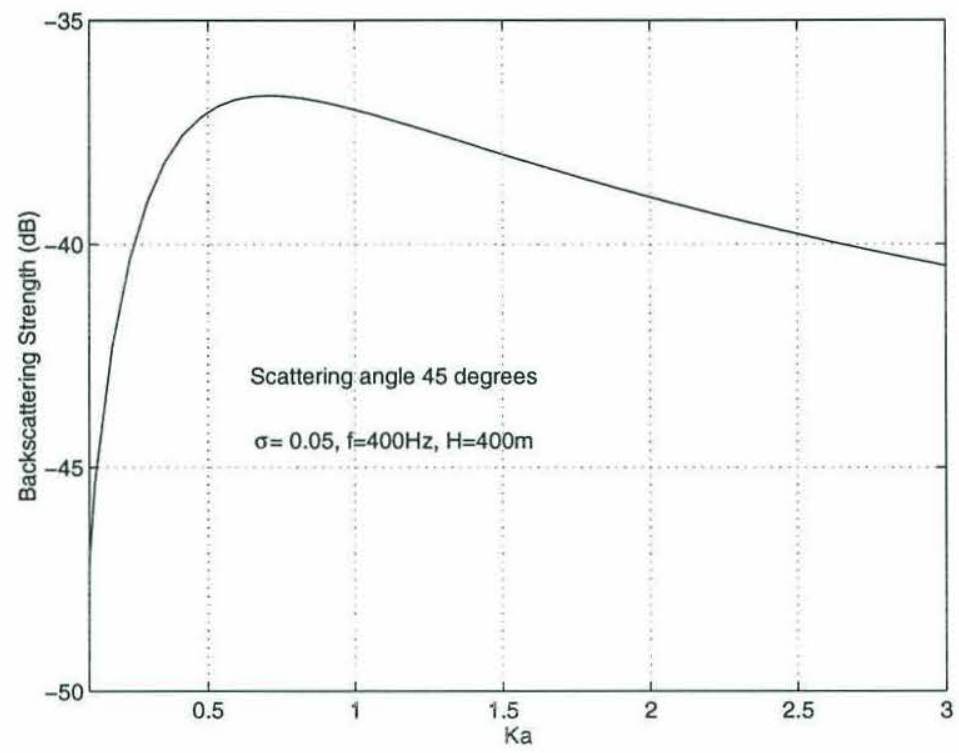

Figure 3-6: Backscattering from sound speed variations with a 2-D isotropic exponential correlation function.

scattering angles. We would expect that the backscattering strength has a different decaying rate versus scattering grazing angle for a Gaussian correlation function and an exponential correlation function as well.

For a correlation function with a general power-law type of power spectrum, we have the following backscattering coefficient:

$$
\Sigma(\theta)=\left\{\begin{array}{l}
\frac{k^{4} \nu \sigma^{2} l_{r}^{2}}{\pi\left[1+\left(2 k l_{r} \cos \theta\right)^{2}\right]^{\nu+1}} \nu \neq 0 \\
\frac{k^{4} \sigma^{2} l_{r}^{2}}{\pi\left[1+\left(2 k l_{r} \cos \theta\right)^{2}\right]} \nu=0
\end{array}\right.
$$

Figure 3-7 depicts that the backscattering strength versus $K a$ for $\nu=1$ with $a=l_{r}$. Similar to the random field with an exponential correlation function, the backscattering strength decays slowly when $K a$ increases.

If we plot together the backscattering strength for the above three types of correlation functions, it is evident that the decaying rate versus $K a$ is essentially related to the roll-off rate of their power spectra. It is the roll-off rates that determines the 


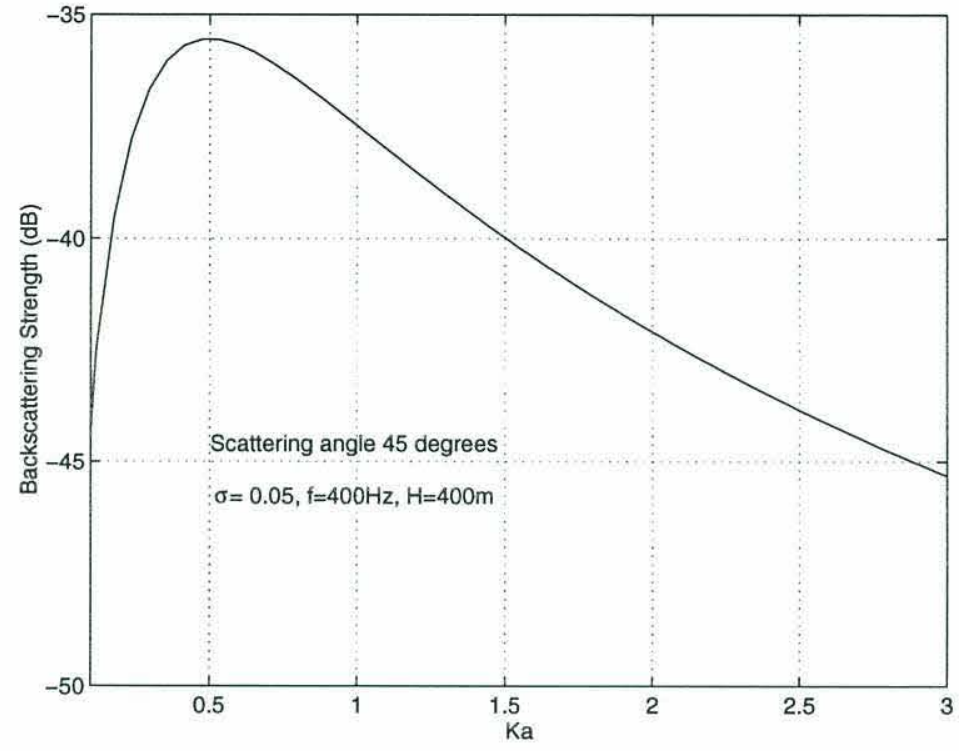

Figure 3-7: Backscattering from sound speed variations with a 2-D isotropic Von Karman function as correlation function $(\nu=1)$.

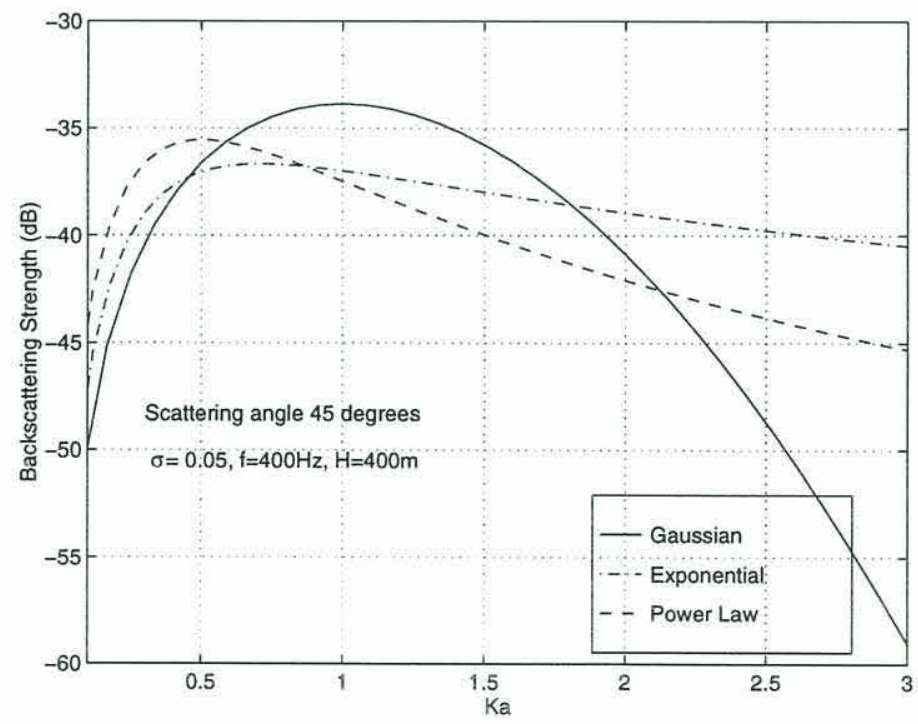

Figure 3-8: Backscattering from sound speed variations with different kinds of 2-D isotropic correlation functions. 
roughness at small length scales therefore the backscattering at large $K a$.

\subsubsection{Backscattering from 2-D Anisotropic Random Field}

The effect of anisotropy on monostatic backscattering will be studied in the following. By anisotropic we mean that the correlation length $l_{x} \neq l_{y}$. Since the scenario with a co-located point source and receiver is of interest in this work, an annulus would be insonified for each scattering angle.

In the case of a Gaussian correlation function, the backscattering coefficient can be derived as

$$
\begin{aligned}
\Sigma(\theta) & =\frac{k^{4} \sigma^{2} l_{x} l_{y}}{4 \pi^{2}} \int_{0}^{2 \pi} \exp \left(-2 k^{2} \cos ^{2} \theta\left(l_{x}^{2} \cos ^{2} \phi+l_{y}^{2} \sin ^{2} \phi\right)\right) d \phi \\
& =\frac{k^{4} \sigma^{2} l_{x} l_{y}}{2 \pi} \exp \left(-k^{2} \cos ^{2} \theta\left(l_{x}^{2}+l_{y}^{2}\right)\right) I_{0}\left(k^{2} \cos ^{2} \theta\left(l_{x}^{2}-l_{y}^{2}\right)\right),
\end{aligned}
$$

where $\phi$ is the azimuthal angle and $I_{0}$ represents the zeroth order modified Bessel function of the first kind. Figure 3-9 shows the backscattering strength versus grazing angle for different combinations of horizontal correlation lengths in the $x$ and $y$ directions. It appears that slopes of backscattering strength versus grazing angle are different for different combinations. In the isotropic case, a random field with $l_{r}=0.6 \mathrm{~m}$ is more effective in backscattering than that with $l_{r}=1 \mathrm{~m}$ and further with

$l_{r}=2 \mathrm{~m}$. Therefore, on the one hand, a random field with $l_{y}=0.6 \mathrm{~m}$ would improve the overall backscattering ability compared to one with $l_{y}=1 \mathrm{~m}$. On the other hand, a random field with $l_{y}=2 m$ would have a negative impact on total backscattering. The azimuthal dependence cannot be discerned because of the omnidirectional source and receiver.

For an anisotropic exponential correlation function, the backscattering coefficient 


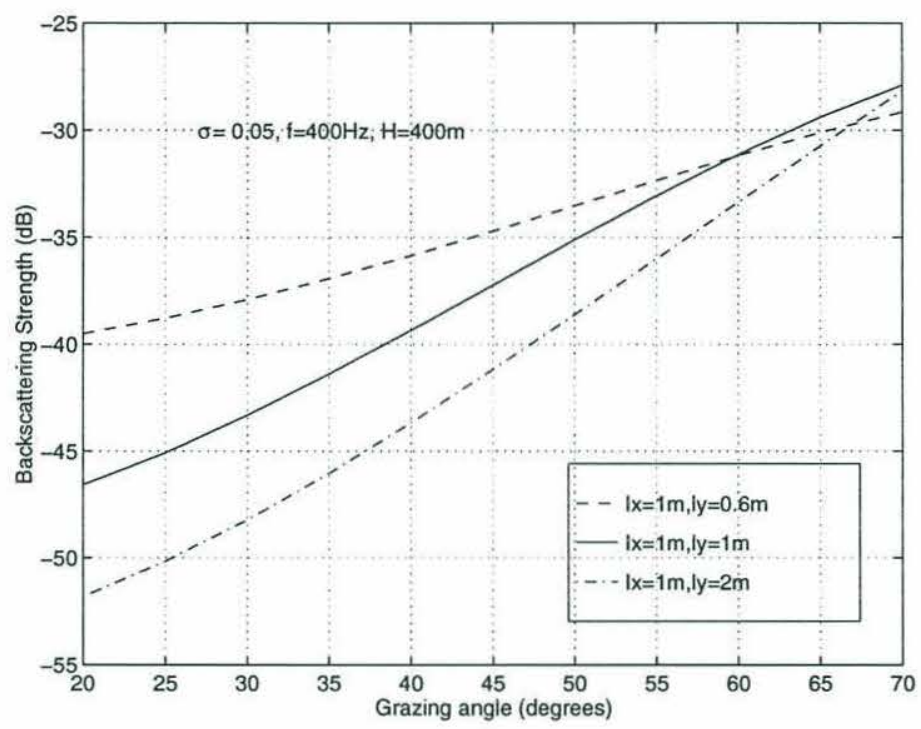

Figure 3-9: Backscattering from sound speed variations with a 2-D anisotropic Gaussian correlation function.

is

$$
\Sigma(\theta)=\frac{k^{4} \sigma^{2} l_{x} l_{y}}{2 \pi^{2}} \int_{0}^{2 \pi} \frac{d \phi}{\left[1+\left(2 k l_{x} \cos \theta \cos \phi\right)^{2}\right]\left[1+\left(2 k l_{y} \cos \theta \sin \phi\right)^{2}\right]} .
$$

What we see in Fig. 3-10 is similar to that in Fig. 3-9. However, the differences among three combinations are not as big as that for a Gaussian distribution. From Fig. 3-8, one can notice that the decay rate versus $K a$ is much smaller for an exponential distribution than for a Gaussian distribution. As a result, the change of correlation length in one direction will not have as strong an influence.

For an anisotropic correlation function specified by Eq.3.32, the backscattering coefficient becomes

$$
\Sigma(\theta)= \begin{cases}\frac{k^{4} \nu \sigma^{2} l_{x} l_{y}}{2 \pi^{2}} \int_{0}^{2 \pi} \frac{d \phi}{\left[1+\left(2 k l_{x} \cos \theta \cos \phi\right)^{2}+\left(2 k l_{y} \cos \theta \sin \phi\right)^{2}\right]^{\nu+1}} \quad \nu \neq 0 \\ \frac{k^{4} \sigma^{2} l_{x} l_{y}}{2 \pi^{2}} \int_{0}^{2 \pi} \frac{d \phi}{\left[1+\left(2 k l_{x} \cos \theta \cos \phi\right)^{2}+\left(2 k l_{y} \cos \theta \sin \phi\right)^{2}\right]} & \nu=0\end{cases}
$$

As can be seen in Fig. 3-11, the differences in backscattering strength due to the 


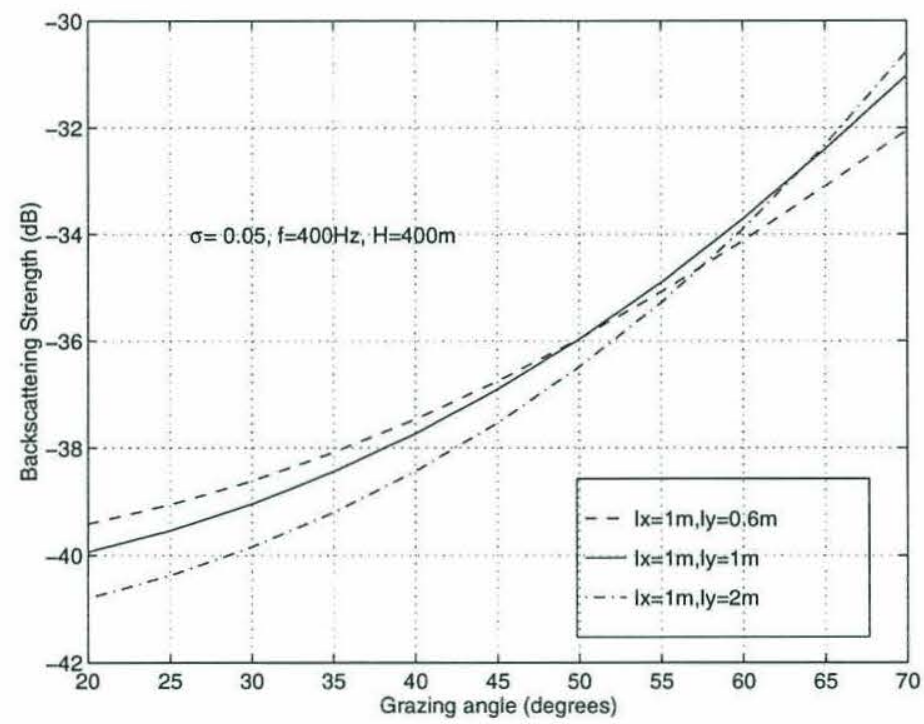

Figure 3-10: Backscattering from sound speed variations with a 2-D anisotropic exponential correlation function.

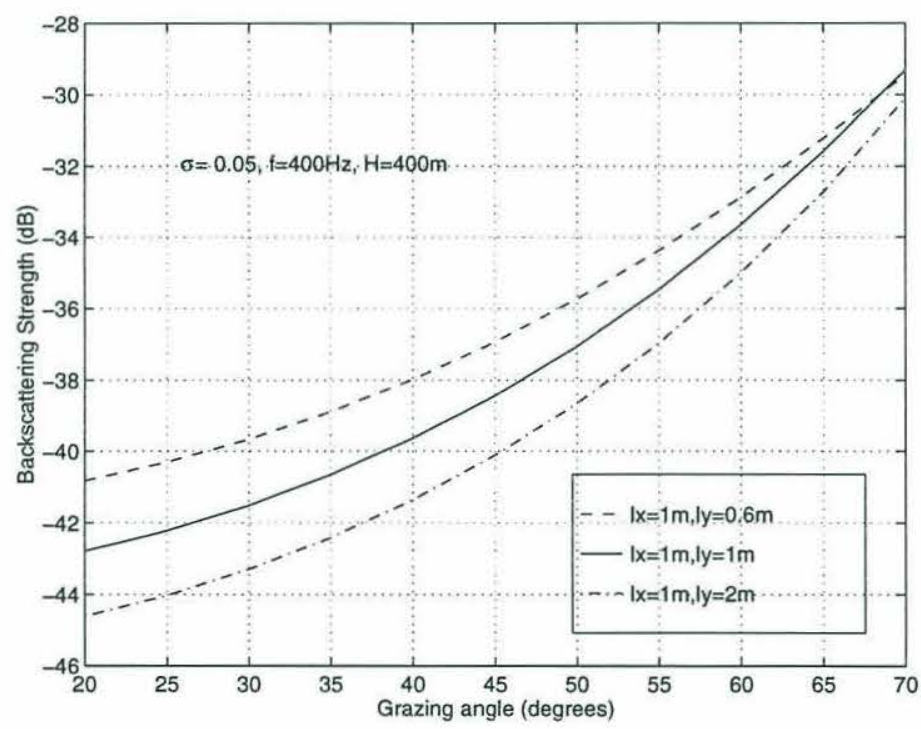

Figure 3-11: Backscattering from sound speed variations with a 2-D anisotropic Von Karman function as the correlation function $(\nu=1)$. 
change of correlation length in the $y$ direction are larger than that with an exponential distribution but smaller than that with a Gaussian distribution. This is consistent with the fact that the decay rate of the backscattering strength versus $K a$ in this case is larger than that of an exponential distribution but smaller than that of a Gaussian distribution.

\subsubsection{Effect of the Vertical Correlation}

For the sake of simplicity, the random sound speed and density variations are sometimes modeled as vertically uncorrelated [7]. What we will show next is the difference in backscattering strength versus grazing angle for vertically correlated and uncorrelated random fields. As we discussed in the previous chapter, the scheme to characterize volume backscattering using equivalent surface scattering strength versus grazing angle is only valid when the scattering layer is thin with respect to the wavelength and the source/receiver are far away. The environmental parameters will be chosen to fulfill this requirement. In the following, we will present only analytic solutions for backscattering from random fields with an isotropic Gaussian or exponential distribution horizontally and exponential distribution vertically.

With an exponential correlation function in the vertical direction, the backscattering coefficient for a horizontally isotropic Gaussian distribution is

$$
\Sigma(\theta)=\frac{k^{4} \sigma^{2} l_{r}^{2} l_{z} D}{\pi\left[1+\left(2 k l_{z} \sin \theta\right)^{2}\right]} \exp \left(-2 k^{2} l_{r}^{2} \cos ^{2} \theta\right),
$$

where $l_{z}$ is the vertical correlation length and $D$ is the layer thickness. For a horizontally isotropic exponential distribution, the backscattering coefficient becomes

$$
\Sigma(\theta)=\frac{k^{4} \sigma^{2} l_{r}^{2} l_{z} D}{\pi\left[1+\left(2 k l_{z} \sin \theta\right)^{2}\right]\left[1+\left(2 k l_{r} \cos \theta\right)^{2}\right]^{3 / 2}} .
$$

It is evident from the above two equations that vertical correlation does affect the 


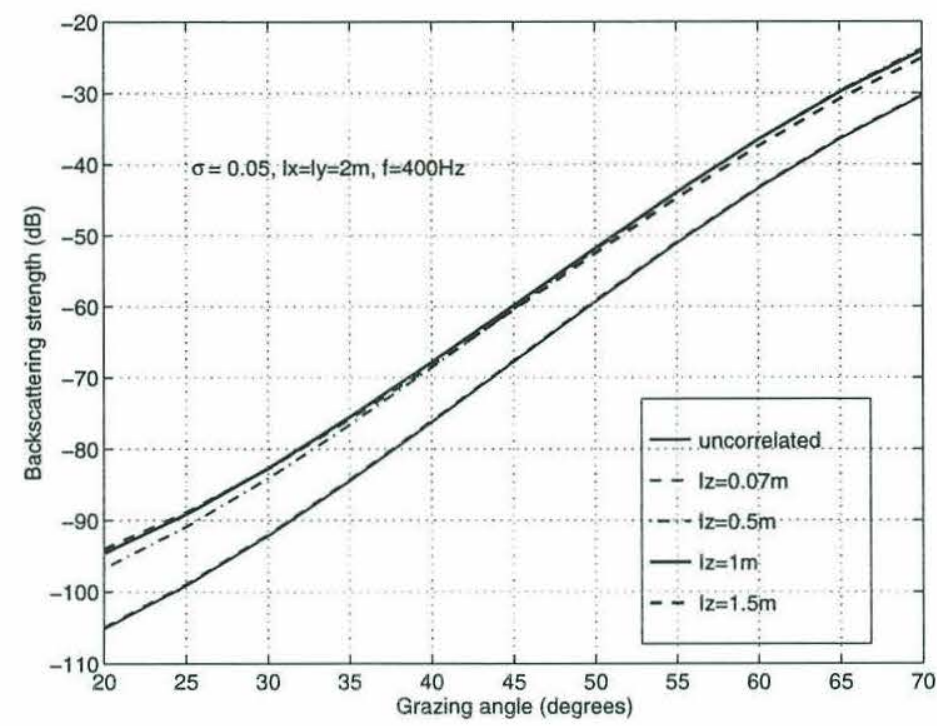

Figure 3-12: Backscattering from sound speed variations with an isotropic Gaussian distribution horizontally and an exponential correlation vertically $\left(\sigma=0.05, l_{x}=l_{y}=\right.$ $2 m, f=400 H z, H=400 m, D=7.5 m)$.

angular dependence of the backscattering strength. The effect is insignificant when $\left(2 k l_{z} \sin \theta\right)^{2} \ll 1$, which can be approximated by a vertically uncorrelated model. However, this condition can be satisfied only at very low frequency and small vertical correlation length. Otherwise, such an effect should not be ignored. When $\left(2 k l_{z} \sin \theta\right)^{2} \gg 1$, small changes in $l_{z}$ will not have strong influences on the angular dependence but only the overall levels of backscattering strengths. While with $\left(2 k l_{z} \sin \theta\right)^{2} \approx 1$, we would expect that the angular dependence of the backscattering strength to be very sensitive to the change of $k l_{z}$.

Figure 3-12 and Fig. 3-13 are two examples to show the effect of vertical correlation. Figure 3-12 depicts the backscattering strength for a 7.5m thick random layer with a Gaussian correlation horizontally. Figure 3-13 is for an exponential distribution horizontally. The correlation lengths $l_{x}$ and $l_{y}$ are chosen to be both $2 \mathrm{~m}$. The standard deviation of sound speed variations is $5 \%$ and the frequency is $400 \mathrm{~Hz}$. We present the backscattering strength curves for different vertical correlation lengths $l_{z}$. In both plots, there is a thin solid line representing the uncorrelated case, for 


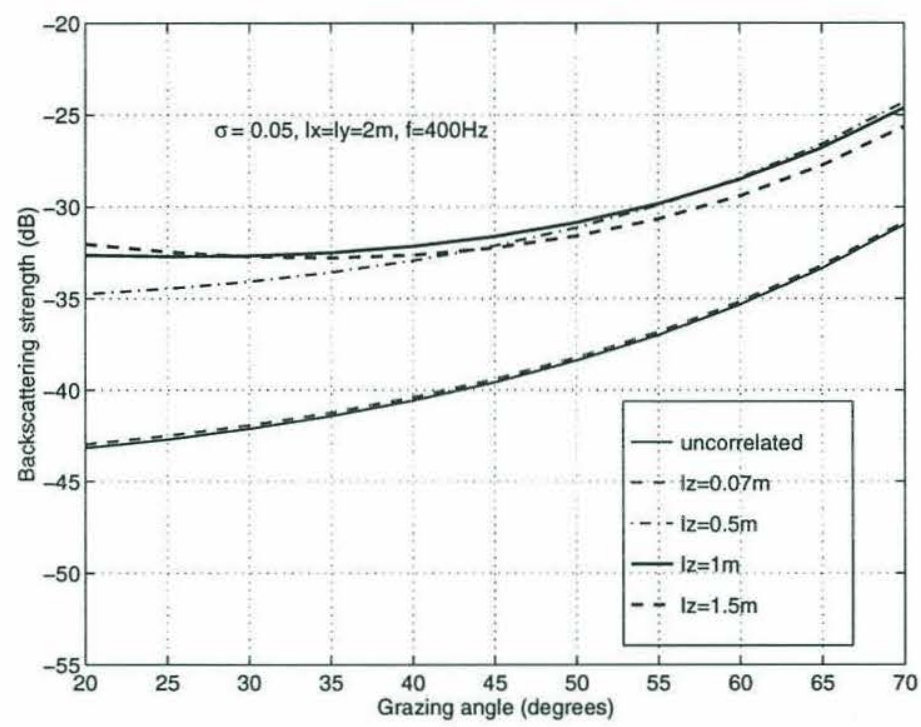

Figure 3-13: Backscattering from sound speed variations with an isotropic exponential distribution horizontally and an exponential correlation vertically $\left(\sigma=0.05, l_{x}=l_{y}=\right.$ $2 m, f=400 \mathrm{~Hz}, \mathrm{H}=400 \mathrm{~m}, \mathrm{D}=7.5 \mathrm{~m})$.

which we use the solutions of a 2-D isotropic distribution. The justification for this comes from the fact that the 3-D solution will not converge to the uncorrelated case with $l_{z}=0$ due to the discontinuity of the exponential correlation function discussed earlier. Fortunately, what interests us most here is the angular dependence of the backscattering strength, which is well embedded in the 2-D solution. As can be seen from both figures, the angular dependence of the backscattering strength for $l_{z}=0.07 m$ is very close to that of an uncorrelated model. However, as $l_{z}$ increases, we can see the backscattering strength level increases for smaller grazing angles, which cannot be predicted by a vertically uncorrelated model. In Fig. 3-8, the backscattering strengths possess peak values for certain $K a$. Similar behavior is expected for the backscattering strength versus $k_{z} l_{z}$, where $k_{z}$ is the vertical wavenumber. The backscattering strength includes contributions from both the horizontal correlation and vertical correlation. As the grazing angle decreases, the horizontal wavenumber $K$ increases and the vertical wavenumber $k_{z}$ decreases. The contribution due to the horizontal correlation will decrease since $K a$ increases, i.e., moving away from the peak, while the contribution from the vertical correlation might increase because of a 
smaller $k_{z} l_{z}$. As a result, there is a possibility for the backscattering strength curve to ascend at small grazing angles, which would not happen for a vertically uncorrelated random medium.

In conclusion, modeling the vertical correlation of the random sound speed and density variations is important in order to capture the full scope of the scattered field, which has justified our efforts to model backscattering from 3-D random volume inhomogeneities in the sediment. 


\section{Chapter 4}

\section{Generation of}

\section{Azimuthally-Summed 3-D Random Sound Speed Variations}

\subsection{Statement of the Problem}

The objective of this research is to develop a model that can account for monostatic backscattering from 3-D volume inhomogeneities.

The scenario of a water layer on top of the sediment layers is considered here with source/receiver co-located in the water column. By applying the method of small perturbation and the Born approximation, the scattered field $P^{s}$ due to sound speed perturbation ( $\beta=0$ here for simplicity) in the sediment volume can be expressed as (cf. Eq.3.14):

$$
P^{s}(\omega, \mathbf{R})=A m \int_{V_{1}} \epsilon\left(\mathbf{R}_{\mathbf{1}}\right)\left(2 k_{1}^{2} G^{2}\left(\mathbf{R}, \mathbf{R}_{\mathbf{1}}\right)\right) d V_{1} .
$$

where $A$ is the sound strength and $m$ is the density ratio between the top of the sediment layer and the water, $\omega$ is the radial frequency, $\mathbf{R}$ and $\mathbf{R}_{\mathbf{1}}$ are the source/receiver position and scatterer position, respectively. The relative sound speed perturbation 
is represented by $\epsilon=\delta c / c_{0}, k_{1}$ is the background wavenumber in the sediment, $V_{1}$ is the scattering volume and $G\left(\mathbf{R}, \mathbf{R}_{\mathbf{1}}\right)$ stands for the Green's function between the scatterer and the receiver. If the above integral is carried out in cylindrical coordinates, $d V_{1}$ can be expanded as $r d r d \phi d z$. By observing the above integral, it can be seen that only $\epsilon$ is dependent on $\phi$. So instead of generating $\epsilon$ in 3-D, if

$$
\eta(r, z)=\int_{0}^{2 \pi} \epsilon(r, \phi, z) d \phi,
$$

can be generated, the $3-\mathrm{D}$ problem is reduced to a 2-D problem. Since the scattering intensity is of most interest to us, the generated sound speed perturbation should possess the desired second-order statistics.

As for the random field generation, the algorithms can be catergorized into two classes: one that is implemented in the spatial domain and another that is implemented in the spectral domain. The spectral method [19] is more popular than the spatial method in the underwater acoustics community because of its efficiency in generating large, multi-dimensional fields. It was applied by Thorsos [67] in his study of rough surface scattering from a 1-D Gaussian random surface. The 2-D Goff-Jordan power law spectrum [66] is also extensively used to simulate the seafloor $[7,68]$. Nevertheless, the literature on 3-D random field generation can hardly be found in underwater acoustics, in contrast to the large number of papers in water resource research literature $[69,70,71]$. The reason may rest on the fact that the computational requirement is too big for the scale of the random field that we are dealing with in underwater acoustics. To serve the purpose of this research, the spectral method is used because of its simplicity and the availability of analytical forms of different power spectral density functions to describe the sound speed perturbation. 


\subsection{Formulas}

The spectral method for generating an azimuthally-summed 3-D random field $\eta(r, z)$ will be presented in this section. For the sake of simplicity, generation of azimuthallysummed 2-D random fields will be studied first. In other words, the dimension $z$ (corresponding to the vertical direction) is dropped for the time being. However, it can be easily added to the formulas later.

In Cartesian coordinates, the power spectral density $W$ can be obtained by

$$
W\left(k_{x}, k_{y}\right)=\lim _{L \rightarrow \infty} \frac{1}{L^{2}}\left\langle\left|\int_{-L / 2}^{L / 2} \int_{-L / 2}^{L / 2} \epsilon(x, y) e^{-i\left(k_{x} x+k_{y} y\right)} d x d y\right|^{2}\right\rangle
$$

where $k_{x}, k_{y}$ are the wavenumbers in the $x, y$ directions, $L^{2}$ is the patch size assuming square area, $\langle\cdot\rangle$ stands for the ensemble average operation and $|\cdot|$ indicates the modulus operation. The power spectral density should be normalized such that

$$
\frac{1}{(2 \pi)^{2}} \int_{-\infty}^{\infty} \int_{-\infty}^{\infty} W\left(k_{x}, k_{y}\right) d k_{x} d k_{y}=\sigma^{2}
$$

where $\sigma$ is the standard deviation of the sound speed perturbation. Equation 4.2 can be proved to satisfy the relation [72]

$$
W\left(k_{x}, k_{y}\right)=\int_{-\infty}^{\infty} \int_{-\infty}^{\infty} C\left(\xi_{x}, \xi_{y}\right) e^{-i\left(k_{x} \xi_{x}+k_{y} \xi_{y}\right)} d \xi_{x} d \xi_{y}
$$

where $C\left(\xi_{x}, \xi_{y}\right)$ is the correlation function and $\xi_{x}, \xi_{y}$ are the lag distances between two points in the $x$ and $y$ directions. The consistency between Eq.4.2 and Eq.4.4 has an important implication. It guarantees that if the power spectral density of the generated random field, estimated through Eq.4.2, converges to the desired one, the generated random field would have the desired correlation function according to Eq.4.4, and therefore the desired second-order statistics. 
According to Eq.4.2, a random field can be generated by

$$
\epsilon(x, y)=\frac{1}{(2 \pi)^{2}} \int_{-\infty}^{\infty} \int_{-\infty}^{\infty} \sqrt{W\left(k_{x}, k_{y}\right) L^{2}} R_{n}\left(k_{x}, k_{y}\right) e^{i\left(k_{x} x+k_{y} y\right)} d k_{x} d k_{y} .
$$

Here the random number $R_{n}$ fits the distribution $R_{n}\left(k_{x}, k_{y}\right) \sim[N(0,1)+i N(0,1)] / \sqrt{2}$, where $N(0,1)$ stands for a Normal distribution with zero mean and standard deviation 1. In order for $\epsilon(x, y)$ to be real, $R_{n}\left(k_{x}, k_{y}\right)$ has to be conjugate symmetric between the first and the third quadrants as well as the second and the fourth quadrants. If the following coordinate transformation

$$
\left\{\begin{array} { l } 
{ k _ { x } = k _ { r } \operatorname { c o s } \theta } \\
{ k _ { y } = k _ { r } \operatorname { s i n } \theta }
\end{array} \text { and } \left\{\begin{array}{l}
x=r \cos \phi \\
y=r \sin \phi
\end{array},\right.\right.
$$

is performed, Eq.4.5 will become

$$
\epsilon(r, \phi)=\frac{1}{(2 \pi)^{2}} \int_{0}^{\infty} \int_{0}^{2 \pi} \sqrt{W\left(k_{r}, \theta\right) L^{2}} R_{n}\left(k_{r}, \theta\right) e^{i k_{r} r \cos (\theta-\phi)} k_{r} d k_{r} d \theta
$$

The equation for $\eta(r)$ will be

$$
\int_{0}^{2 \pi} \epsilon(r, \phi) d \phi=\frac{1}{2 \pi} \int_{0}^{\infty} \int_{0}^{2 \pi} \sqrt{W\left(k_{r}, \theta\right) L^{2}} R_{n}\left(k_{r}, \theta\right) k_{r} J_{0}\left(k_{r} r\right) d k_{r} d \theta .
$$

We point out that $\eta(r)$ is a nonstationary process. Here $\Delta \phi=2 \pi / N(r)$ and $N(r)$ is the number of scatterers around the circle with radius $r$. Assuming constant density for the scatterers, $N(r)$ will increase proportionally to $r$. Therefore, the variance of $\eta(r)$ will decrease accordingly, which makes $\eta(r)$ a nonstationary process. Collecting the items on the right-hand side which depend on $\theta$, it gives

$$
F\left(k_{r}\right)=\frac{1}{2 \pi} \int_{0}^{2 \pi} \sqrt{W\left(k_{r}, \theta\right) L^{2}} R_{n}\left(k_{r}, \theta\right) d \theta .
$$

Changing the continuous integral to a discrete sum and using the relation

$$
\frac{(2 \pi)^{2}}{L^{2}}=\Delta k_{x} \Delta k_{y}=k_{r} \Delta k_{r} \Delta \theta \quad\left(k_{r} \neq 0\right)
$$


where $\Delta(\cdot)$ represents the sampling interval, Eq.4.9 becomes

$$
F\left(k_{r}\right)=\sum_{i=1}^{M\left(k_{r}\right)} \sqrt{\frac{W\left(k_{r}, \theta_{i}\right) \Delta \theta}{k_{r} \Delta k_{r}}} R_{n}\left(k_{r}, \theta_{i}\right)
$$

where $\theta_{i}=i \Delta \theta$ and $M\left(k_{r}\right)$ is the number of samples around a circle of radius $k_{r}$. The value of $F$ at $k_{r}=0$ can be arbitrary as long as it is finite because it will be multiplied by $k_{r}$, which is 0 here, in Eq.4.8. $F\left(k_{r}\right)$ can be treated as a new random variable with mean 0 and variance

$$
\sum_{i=1}^{M\left(k_{r}\right)} \frac{W\left(k_{r}, \theta_{i}\right) \Delta \theta}{k_{r} \Delta k_{r}} \quad\left(k_{r} \neq 0\right)
$$

since $R_{n}\left(k_{r}, \theta_{i}\right)$ are independent of each other and each has the variance of 1 . Therefore, $F\left(k_{r}\right)$ can be replaced by

$$
\sqrt{\frac{\int_{0}^{2 \pi} W\left(k_{r}, \theta\right) d \theta}{k_{r} d k_{r}}} R_{n}\left(k_{r}\right) . \quad\left(k_{r} \neq 0\right)
$$

where $R_{n}\left(k_{r}\right) \sim N(0,1)$. Notice that here $R_{n}\left(k_{r}\right)$ is a real number due to the fact that the imaginary parts of $R_{n}\left(k_{r}, \theta\right)$ have been cancelled because of the earlier mentioned property of conjugate symmetry. Eq.4.8 now becomes

$$
\eta(r)=\sum_{i=1}^{\infty} \sqrt{\frac{\int_{0}^{2 \pi} W\left(k_{r_{i}}, \theta\right) d \theta}{k_{r_{i}} \Delta k_{r}}} R_{n}\left(k_{r_{i}}\right) k_{r_{i}} J_{0}\left(k_{r_{i}} r\right) \Delta k_{r}
$$

where $k_{r_{i}}=i \Delta k_{r}$.

As mentioned above, the right second-order statistics of generated $\eta(r)$ is guaranteed. From Eq.4.4, the correlation function of $\epsilon$ in Cartesian coordinates can be written as

$$
\begin{aligned}
& \left\langle\epsilon\left(x_{1}, y_{1}\right) \epsilon^{*}\left(x_{2}, y_{2}\right)\right\rangle= \\
& \frac{1}{(2 \pi)^{2}} \int_{-\infty}^{\infty} \int_{-\infty}^{\infty} W\left(k_{x}, k_{y}\right) e^{i\left[k_{x}\left(x_{2}-x_{1}\right)+k_{y}\left(y_{2}-y_{1}\right)\right]} d k_{x} d k_{y} .
\end{aligned}
$$


where $(*)$ stands for conjugate operation. If the coordinate systerm is again changed to polar coordinates, it will result in

$$
\begin{aligned}
& \left\langle\epsilon\left(r_{1}, \phi_{1}\right) \epsilon^{*}\left(r_{2}, \phi_{2}\right)\right\rangle= \\
& \quad \frac{1}{(2 \pi)^{2}} \int_{0}^{\infty} \int_{0}^{2 \pi} W\left(k_{r}, \theta\right) e^{i k_{r}\left[r_{1} \cos \left(\theta-\phi_{1}\right)-r_{2} \cos \left(\theta-\phi_{2}\right)\right]} k_{r} d k_{r} d \theta .
\end{aligned}
$$

Therefore, the correlation function of $\eta(r)$ is

$$
\begin{aligned}
\left\langle\eta\left(r_{1}\right) \eta^{*}\left(r_{2}\right)\right\rangle & =\int_{0}^{2 \pi} \int_{0}^{2 \pi}\left\langle\epsilon\left(r_{1}, \phi_{1}\right) \epsilon^{*}\left(r_{2}, \phi_{2}\right)\right\rangle d \phi_{1} d \phi_{2} \\
& =\int_{0}^{\infty}\left[\int_{0}^{2 \pi} W\left(k_{r}, \theta\right) d \theta\right] k_{r} J_{0}\left(k_{r} r_{1}\right) J_{0}\left(k_{r} r_{2}\right) d k_{r} .
\end{aligned}
$$

It can be seen that $\eta(r)$ generated by Eq.4.11 satisfies the above equation. Let us denote

$$
W_{s}=\int_{0}^{2 \pi} W\left(k_{r}, \theta\right) d \theta,
$$

which can be considered the radial power spectral density. It is instructive to compute the spectral estimates $\hat{W}_{s}$ from the random realizations of sound speed perturbations for comparison with $W_{s}$. In continuum notation, we calculate

$$
\hat{W}_{s}=\frac{2 \pi k_{r}}{L}\left\langle\left|\int_{0}^{L / 2} w(r) \eta(r) r J_{0}\left(k_{r} r\right) d r\right|^{2}\right\rangle,
$$

where $w(r)$ is a real non-negative weighting or window function normalized such that

$$
\frac{2}{L} \int_{0}^{L / 2} w^{2}(r) d r=1
$$

The weighting function is chosen to improve the spectral estimation by reducing an effect known as spectral leakage. So if the estimated $\hat{W}$ agrees with the desired $W_{s}$, the generated $\eta(r)$ can be considered satisfactory. This equation will be used later in numerical experiments as the criterion to determine the acceptability of the generated random field. 
Eq.4.11 can be easily extended to the generation of a 3-D azimuthally-summed random field in cylindrical coordinates:

$$
\begin{aligned}
\eta(r, z)=\frac{1}{2 \pi} \sum_{m=0}^{\infty} \sum_{n=-\infty}^{\infty} \sqrt{\frac{D \int_{0}^{2 \pi} W\left(k_{r_{m}}, k_{z_{n}}, \theta\right) d \theta}{k_{r_{m}} \Delta k_{r}}} \\
R_{n}\left(k_{r_{m}}, k_{z_{n}}\right) k_{r_{m}} J_{0}\left(k_{r_{m}} r\right) e^{i k_{z_{n}} z} \Delta k_{r} \Delta k_{z},
\end{aligned}
$$

where $k_{r_{m}}=m \Delta k_{r}, k_{z_{n}}=n \Delta k_{z}$ and $R_{n}\left(k_{r}, k_{z}\right)$ has to be conjugate symmetric in the $z$ direction in order for $\eta(r, z)$ to be real. The layer thickness is denoted by $D$. This process can be viewed as passing the random series $\eta(r)$ generated according to Eq.4.11 through a filter in the $z$ direction. As for the power spectral density $W\left(k_{r}, k_{z}, \theta\right)$ used in the generation, it can be in any form, including the result of a direct measurement in an experiment.

If the area within a few wavelengths distance from the center is not a concern, Eq.4.17 can be evaluated using a Fourier transform instead of a Hankel transform in the $r$ direction and a Fourier transform in $z$ direction, which is essentially the same as the Fast-Field-Program(FFP) approach introduced by DiNapoli and Deavenport [73]. First the Bessel function $J_{0}\left(k_{r} r\right)$ is expressed in terms of Hankel functions,

$$
J_{0}\left(k_{r} r\right)=\frac{1}{2}\left[H_{0}^{(1)}\left(k_{r} r\right)+H_{0}^{(2)}\left(k_{r} r\right)\right]
$$

where $H_{0}^{(1)}$ and $H_{0}^{(2)}$ are the zeroth order Hankel functions of the first and second kind, respectively. Next, $H_{0}^{(1)}\left(k_{r} r\right)$ and $H_{0}^{(2)}\left(k_{r} r\right)$ are replaced by their asymptotic forms [74]

$$
\begin{aligned}
\lim _{k_{r} r \rightarrow \infty} H_{0}^{(1)}\left(k_{r} r\right) & =\sqrt{\frac{2}{\pi k_{r} r}} e^{i\left(k_{r} r-\frac{\pi}{4}\right)}, \\
\lim _{k_{r} r \rightarrow \infty} H_{0}^{(2)}\left(k_{r} r\right) & =\sqrt{\frac{2}{\pi k_{r} r}} e^{-i\left(k_{r} r-\frac{\pi}{4}\right)},
\end{aligned}
$$


to arrive at the following expression,

$$
\begin{array}{r}
\eta(r, z)=\frac{\Delta k_{z}}{2 \pi} \sqrt{\frac{\Delta k_{r}}{2 \pi r}} \sum_{m=0}^{\infty} \sum_{n=-\infty}^{\infty}\left[e^{-i \frac{\pi}{4}} \sqrt{D \int_{0}^{2 \pi} W\left(k_{r_{m}}, k_{z_{n}}, \theta\right) d \theta} R_{n}\left(k_{r_{m}}, k_{z_{n}}\right)\right. \\
\left.e^{i\left(k_{r_{m}} r+k_{z_{n}} z\right)}+e^{i \frac{\pi}{4}} \sqrt{D \int_{0}^{2 \pi} W\left(k_{r_{m}}, k_{z_{n}}, \theta\right) d \theta} R_{n}\left(k_{r_{m}}, k_{z_{n}}\right) e^{-i\left(k_{r_{m}} r-k_{z_{n}} z\right)}\right] .
\end{array}
$$

In the model/data comparison presented in Chapter 6, our interest is on medium to low grazing angle backscattering. The generated random field close to the center is

going to be discarded anyway. As a result, Eq.4.20 can be exploited to speed up the computation.

\subsection{Numerical Examples}

In this section, azimuthally-summed 2-D random fields are going to be generated. These numerical examples will help verify the methodology that is described above. The reasons that 2-D random fields instead of 3-D random fields are chosen are due to the following considerations: first is simplicity, since it is easier to show the agreement of power spectral densities and correlation functions in 2-D cases; second is the fact that the azimuthal summation is performed only in a horizontal plane.

The situations considered will include isotropic and anisotropic random fields. By isotropic, it means that the correlation lengths in both the $x$ and $y$ directions are the same; by anisotropic, it means that the correlation lengths are different in the $x$ and $y$ directions. In both cases, Gaussian correlation functions will be used. However, it can be regarded as a general assessment of the methodology.

The 2-D Gaussian correlation function in Cartesian coordinates is

$$
C\left(\xi_{x}, \xi_{y}\right)=\sigma^{2} \exp \left(-\frac{1}{2}\left[\frac{\xi_{x}^{2}}{l_{x}^{2}}+\frac{\xi_{y}^{2}}{l_{y}^{2}}\right]\right),
$$

where $\sigma$ is the standard deviation, and $l_{x}$ and $l_{y}$ are the correlation lengths in the $x$ 
and $y$ directions, respectively. The corresponding power spectral density is

$$
W\left(k_{x}, k_{y}\right)=2 \pi \sigma^{2} l_{x} l_{y} \exp \left(-\frac{k_{x}^{2} l_{x}^{2}+k_{y}^{2} l_{y}^{2}}{2}\right) .
$$

\subsubsection{Isotropic Case}

In the isotropic case, $l_{x}=l_{y}=l$. By performing the coordinate transformation from Cartesian coordinates to polar coordinates according to Eq.A.3, Eq.A.2 becomes

$$
W\left(k_{r}, \theta\right)=2 \pi \sigma^{2} l^{2} \exp \left(-\frac{k_{r}^{2} l^{2}}{2}\right) .
$$

Since $W$ is independent of $\theta, W_{s}\left(k_{r}\right)$ is simply $2 \pi W\left(k_{r}\right)$. The correlation function of $\eta(r)$ can be obtained as (see Appendix A)

$$
\left\langle\eta\left(r_{1}\right) \eta^{*}\left(r_{2}\right)\right\rangle=4 \pi^{2} \sigma^{2} I_{0}\left(\frac{r_{1} r_{2}}{l^{2}}\right) \exp \left(-\frac{r_{1}^{2}+r_{2}^{2}}{2 l^{2}}\right),
$$

where $I_{0}$ stands for the zeroth order modified Bessel function of the first kind. The variance at each range $r$ is

$$
\left\langle\eta(r) \eta^{*}(r)\right\rangle=4 \pi^{2} \sigma^{2} I_{0}\left(\frac{r^{2}}{l^{2}}\right) \exp \left(-\frac{r^{2}}{l^{2}}\right) .
$$

It is evident that the variance of $\eta$ depends on $r$, which proves that $\eta(r)$ is a nonstationary process.

Figure 4-1 shows 10 realizations of the generated random sound speed perturbations after being summed up azimuthally. Here the horizontal correlation lengths $l_{x}$ and $l_{y}$ are both $5 \mathrm{~m}$. The standard deviation $\sigma$ is chosen to be $1 \%$. There is a decaying trend of the magnitude over range, which is the result of decaying variances. 


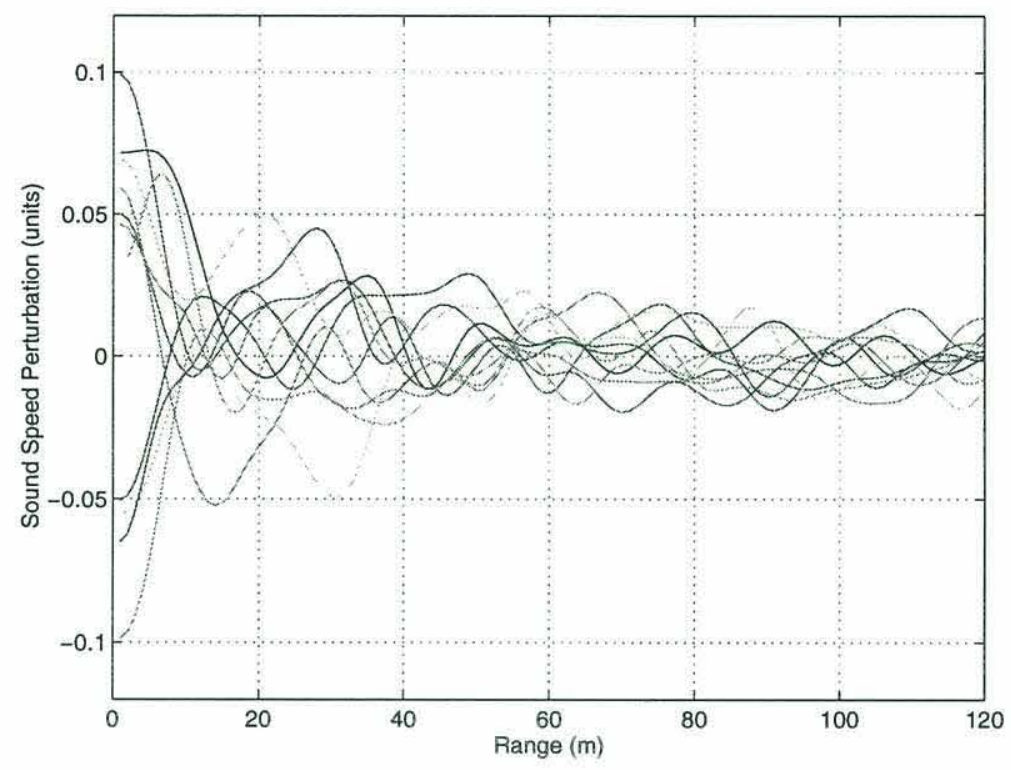

Figure 4-1: 10 realizations of azimuthally-summed 2-D random fields with a Gaussian correlation function and $\sigma=1 \%, l_{x}=l_{y}=5 \mathrm{~m}$

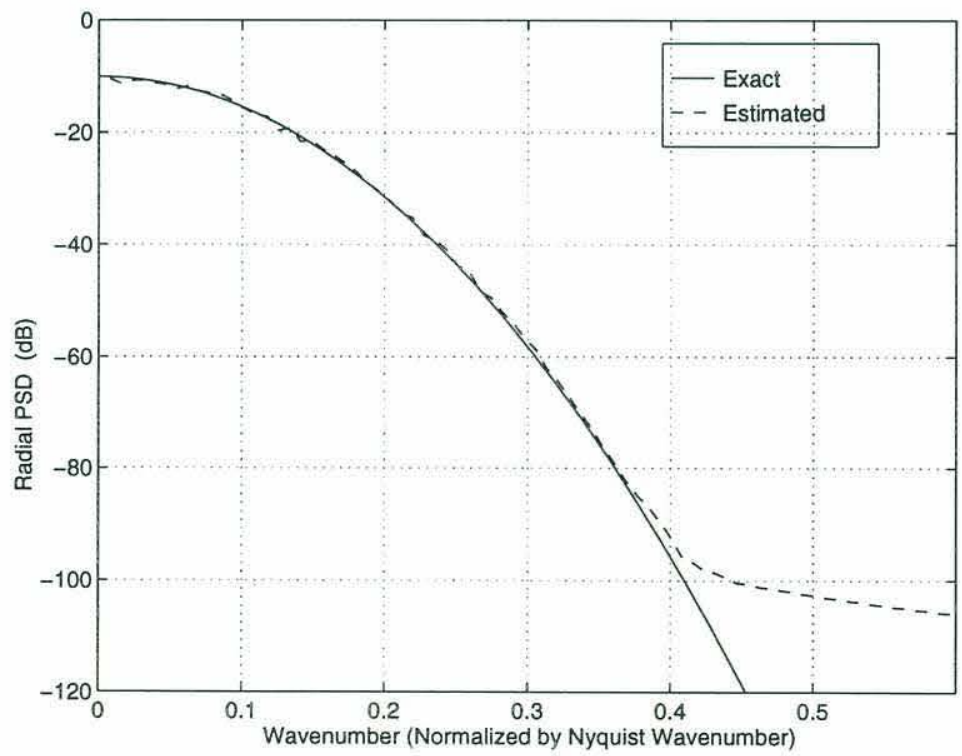

Figure 4-2: Comparison of radial power spectral density estimate with exact radial power spectral density for an ensemble of 100 realizations (isotropic case) 


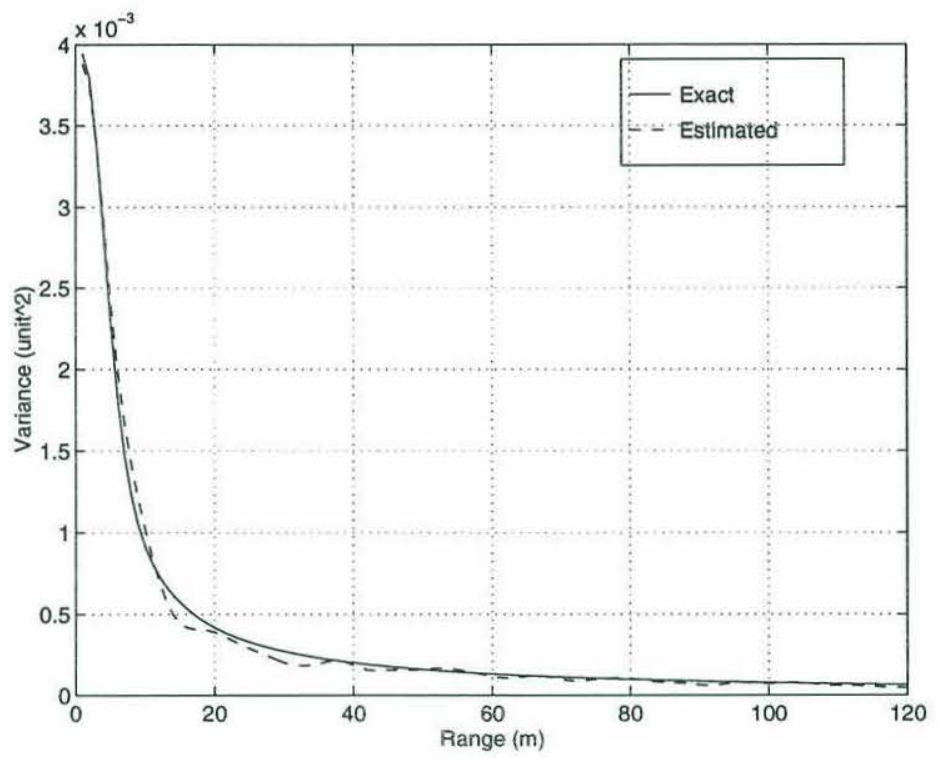

Figure 4-3: Comparison of variance estimate with exact variance for an ensemble of 100 realizations(isotropic case)

A comparison of radial power spectral density estimate $\hat{W}_{s}$ and the exact $W_{s}$ is shown in Fig. 4-2. Here, $\hat{W}_{s}$ is obtained with 100 realizations using the same parameters as in Fig.4-1. A Hanning window is chosen to be the weighting function $w(r)$. Reasonable convergence of $\hat{W}_{s}$ to $W_{s}$ is observed except at low spectral levels. This difference occurs because $w(r)$ still has a small finite value at the endpoints, which is a typical spectral leakage effect.

Fig. 4-3 shows a comparison of the variance estimate of $\eta(r)$ and the exact result obtained through Eq.A.6. The estimate is obtained by

$$
\operatorname{Var}[\eta(r)]=\frac{1}{N_{r l z}-1} \sum_{i=1}^{N_{r l z}} \eta^{2}(r)
$$

where $N_{r l z}$ is the number of realizations used in the estimation. Again, 100 realization has been used. The agreement between the estimate and the exact result is reasonably good, which shows the feasibility of this method. More ensemble averaging will certainly improve the match. 


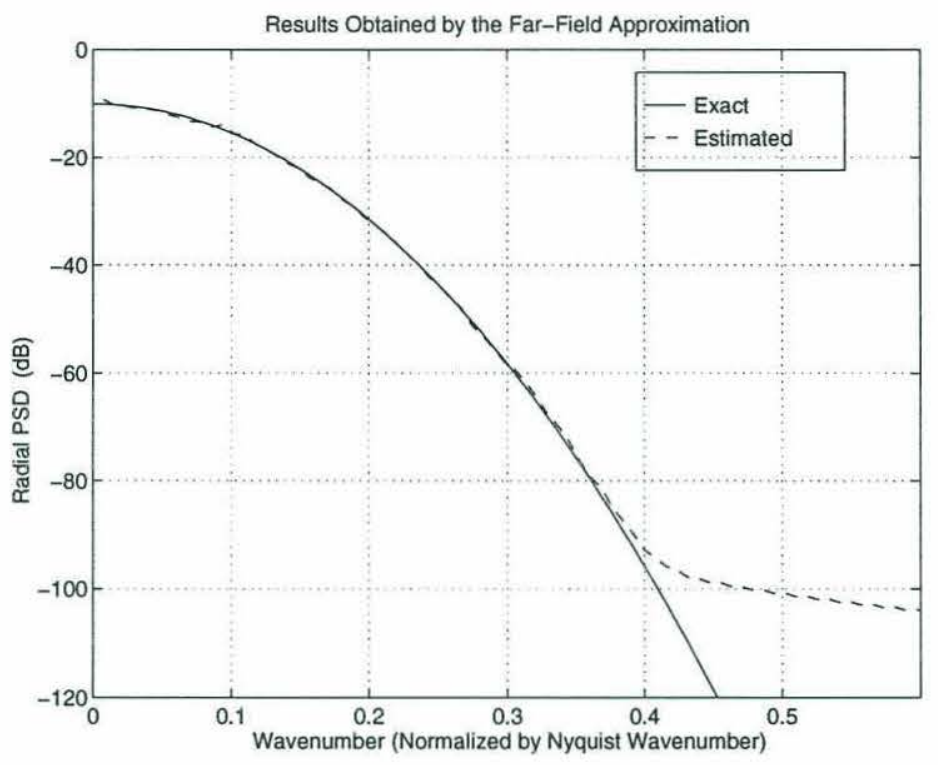

Figure 4-4: Comparison of radial power spectral density estimate with exact power spectral density for an ensemble of 100 realizations using the FFP approach

Fig. 4-4 is the same as Fig. 4-2 except the random fields are generated by the FFP approach described in Eq.4.20. The good fit between the estimated and exact $W_{s}$ indicates the acceptability of this approach.

\subsubsection{Anisotropic Case}

In an anisotropic situation, the coordinate transformation of Eq.A.2 through Eq.A.3 will give

$$
W\left(k_{r}, \theta\right)=2 \pi \sigma^{2} l_{x} l_{y} \exp \left(-\frac{k_{r}^{2}\left(l_{x}^{2} \cos ^{2} \theta+l_{y}^{2} \sin ^{2} \theta\right)}{2}\right) .
$$

As a result,

$$
W_{s}\left(k_{r}\right)=4 \pi^{2} \sigma^{2} I_{0}\left(\frac{k_{r}^{2}\left(l_{x}^{2}-l_{y}^{2}\right)}{4}\right) e^{-\frac{k_{r}^{2}\left(l_{x}^{2}+l_{y}^{2}\right)}{4}}
$$




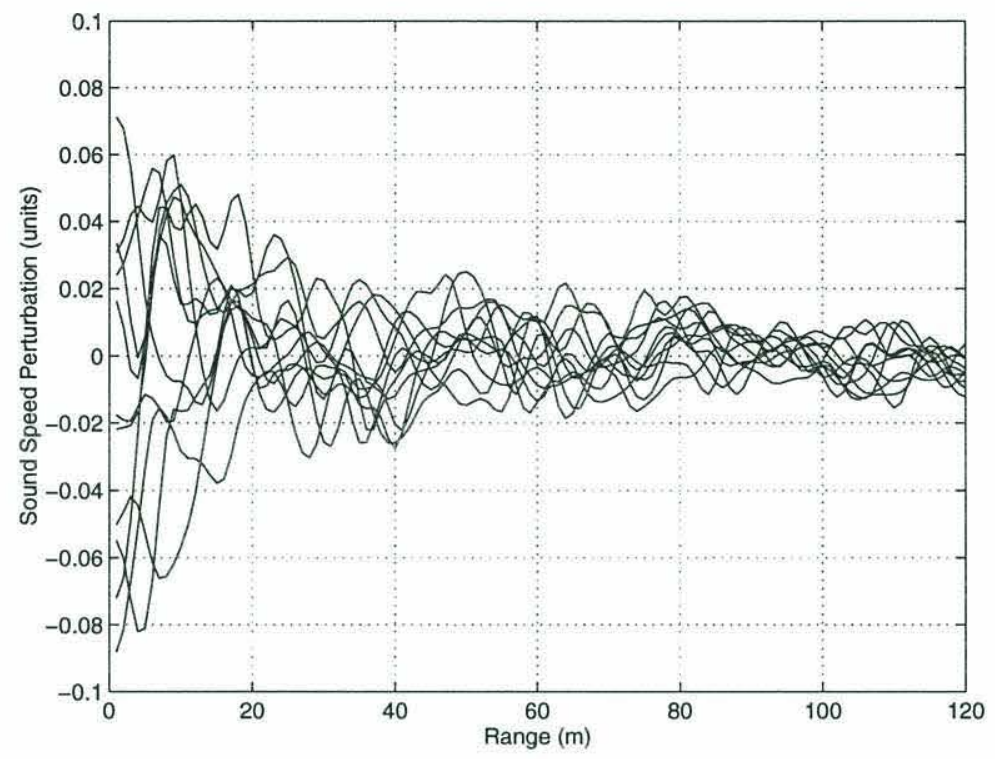

Figure 4-5: 10 realizations of azimuthally-summed 2-D random fields with a Gaussian correlation function and $\sigma=1 \%, l_{x}=2 m, l_{y}=5 m$

(See Appendix A for a detailed derivation). The variance of $\eta(r)$ is

$$
\begin{aligned}
& \operatorname{Var}\{\eta(r)\}= \\
& \sigma^{2} \int_{0}^{2 \pi} \int_{0}^{2 \pi} \exp \left(-\frac{r^{2}}{2 l_{x}^{2} l_{y}^{2}}\left[l_{y}^{2}\left(\cos \phi_{2}-\cos \phi_{1}\right)^{2}+l_{x}^{2}\left(\sin \phi_{2}-\sin \phi_{1}\right)^{2}\right]\right) d \phi_{1} d \phi_{2} .
\end{aligned}
$$

This equation can be evaluated numerically.

Figure 4-5 shows 10 realizations of the generated random sound speed perturbations after being summed up azimuthally. Here the horizontal correlation lengths $l_{x}$ and $l_{y}$ are $2 m$ and $5 m$, respectively. The standard deviation $\sigma$ is again $1 \%$. By comparing Fig.4-5 with Fig. 4-1, one can notice that there are more oscillations over the range in the previous one. This is because the correlation length $l_{x}$ in this anisotropic case is $2 m$ instead of the $5 m$ used in the isotropic case, which means that the correlation of the original 2-D field before azimuthal summation is not as good as that in the isotropic case. This is reflected in the azimuthally-summed field. It will be more evident when the radial power spectral densities are compared later. 


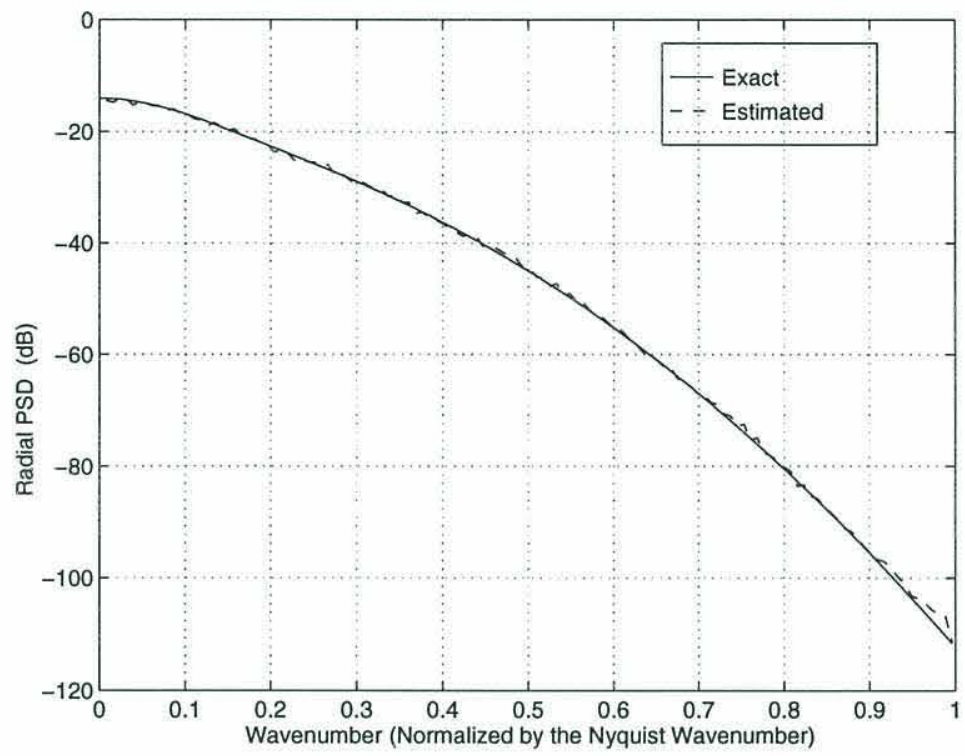

Figure 4-6: Comparison of radial power spectral density estimate with exact radial power spectral density for ensemble of 100 realizations (anisotropic case)

Again, good agreement can be seen between the estimated power spectral density $\hat{W}_{s}$ and the exact $W_{s} . \hat{W}_{s}$ is obtained with 100 realizations using the same parameters as in Fig.4-5. A Hanning window is chosen to be the weighting function $w(r)$. In this particular case, the difference at low spectral level due to spectral leakage cannot be observed since it is out of the plotted wavenumber region. The match between the estimated variance of $\eta(r)$ and the exact one obtained through numerical evaluation of Eq. A.8 is good as well.

Next we compare the radial power spectral density $W_{s}$ for random fields with different correlation lengths $l_{x}$. It can be seen in Fig. 4-8 that the decay rate of power spectral density versus wavenumber is inversely proportional to the correlation length $l_{x}$ when $l_{y}$ is equal. The larger the $l_{x}$, the better the correlation of the original field, the larger the decay rate. From the perspective of spatial-wavenumber relationship, there will be more energy at high wavenumber for poorer correlation, which corresponds to higher oscillation in spatial domain as can be seen in Fig. 4-5 compared to that in Fig. 4-1. 


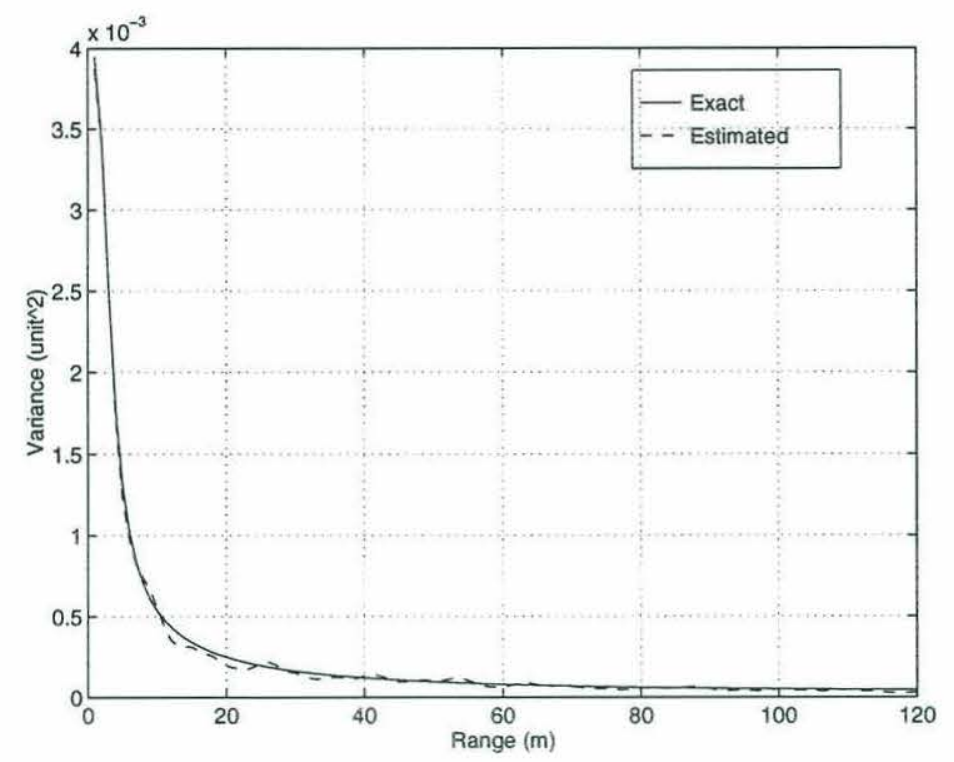

Figure 4-7: Comparison of variance estimate with exact variance for an ensemble of 100 realizations(anisotropic case)

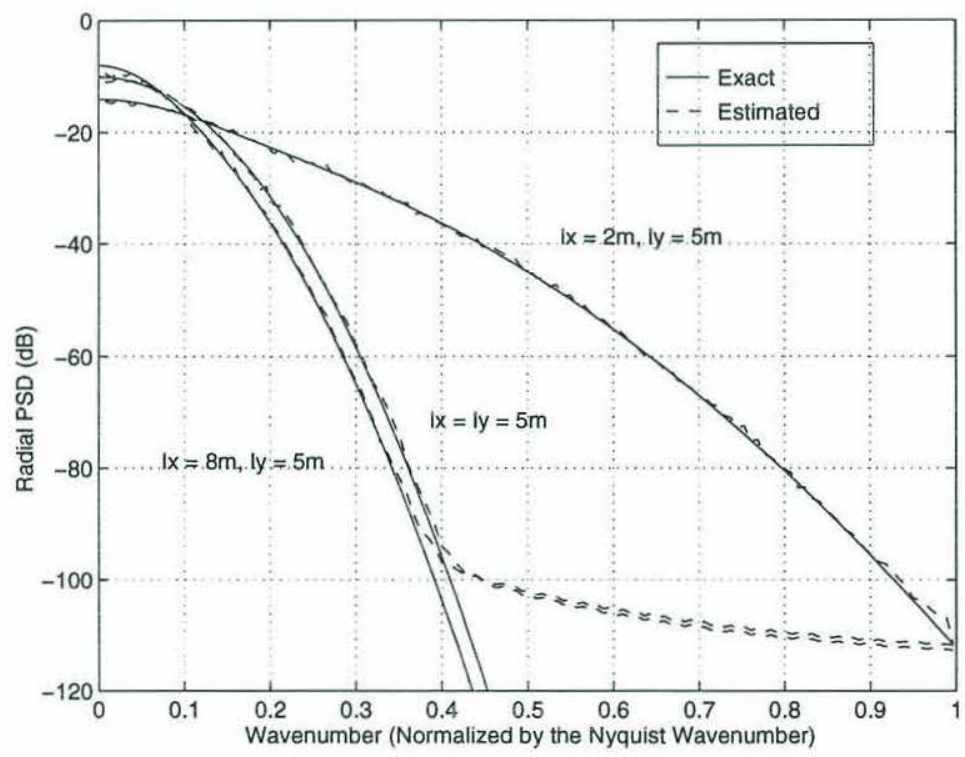

Figure 4-8: Comparison of radial power spectral densities for random fields with the same correlation length $l_{y}$ but different correlation length $l_{x}$. The estimated radial spectral densities are obtained by an ensemble average of 100 realizations 


\subsection{Summary}

The purpose of generating azimuthally-summed random fields is to reduce the computational load of studying monostatic backscattering from 3-D random inhomogeneities by transforming a $3-\mathrm{D}$ problem to a $2-\mathrm{D}$ problem if the source and receiver are colocated. The numerical examples given in the previous section prove that the proposed approach is successful, which will facilitate the synthesis of backscattering time series in the next chapter. 


\section{Chapter 5}

\section{Numerical Simulations of}

\section{Backscattered Time Series}

One of the objectives of this thesis work is to enable the simulation of the backscattered field and backscattering time series due to three-dimensional volume inhomogeneities in the sediment. In Chapter 3, a small perturbation approach was employed to derive the formulations for bottom scattering from sound speed and density variations. In Chapter 4, a scheme was proposed to generate azimuthally-summed threedimensional random fields using the spectral method. With the calculation of the Green's function discussed in Chapter 2 handled by OASES and sound speed and density variations generated by the above-mentioned spectral method, we are prepared to simulate monostatic backscattered fields and backscattering time series at the receiver.

In this chapter, we will describe procedures for generating backscattering time series first. Then the emphasis will be put on comparisons between numerical simulation and analytic solutions. The effect of density variations on backscattering will be studied at the end. 


\subsection{Numerical Simulations}

The keys to the numerical simulation of scattered fields and the scattering time series rest on the following equations (cf. Eq.3.14)

$$
P_{s}\left(\mathbf{R}_{\mathbf{r}}\right)=\int_{v} \epsilon\left(\mathbf{r}^{\prime}\right)\left[(2+\beta) k_{0}^{2}\left(z^{\prime}\right) P_{0}\left(\mathbf{r}^{\prime}\right) G\left(\mathbf{R}_{\mathbf{r}}, \mathbf{r}^{\prime}\right)-\beta\left(\nabla P_{0}\left(\mathbf{r}^{\prime}\right) \cdot \nabla G\left(\mathbf{R}_{\mathbf{r}}, \mathbf{r}^{\prime}\right)\right)\right] d \mathbf{r}^{\prime},
$$

where $\mathbf{R}_{\mathbf{r}}$ stands for the receiver position and $\mathbf{r}^{\prime}$ stands for the scatterer position. The scattering time series $y(t)$ can be obtained by (cf. Eq.3.16)

$$
y(t)=\frac{1}{2 \pi} \int_{-\infty}^{\infty} P_{s}\left(\mathbf{R}_{\mathbf{r}}, \omega\right) S(\omega) \exp (i \omega t) d \omega
$$

where $\omega$ is the radial frequency and $S(\omega)$ is the spectrum of the source signal. The numerical procedures for these are self-evident, i.e.,

- Calculate the Green's function $G$ and the mean field $P_{0}$. For simple cases such as a free-space scenario, analytic solutions are available, while for more complicated environments, some numerical toolboxes (e.g., OASES and Kraken [75]) can be exploited. OASES is our choice in this work.

- Generate azimuthally-summed 3-D random sound speed variations using the spectral method described in Chapter 4. For monostatic backscattering, except for $\epsilon$, all the terms on the RHS of Eq.3.14 are independent of azimuth in a cylindrical coordinate. As a result, we can take advantage of having an azimuthally-summed random field instead of a full 3-D random field because of the difficulty of meeting the computational requirement for the generation of the latter.

- Obtain the monostatic backscattered field using Eq.3.14. If the ultimate goal is to simulate a backscattering time series, scattered fields for many frequencies have to be calculated. Subsequently, $G$ and $P_{0}$ have to be computed for multiple frequencies. Meanwhile, random sound speed variations need only to be generated once because they are frequency-independent. 

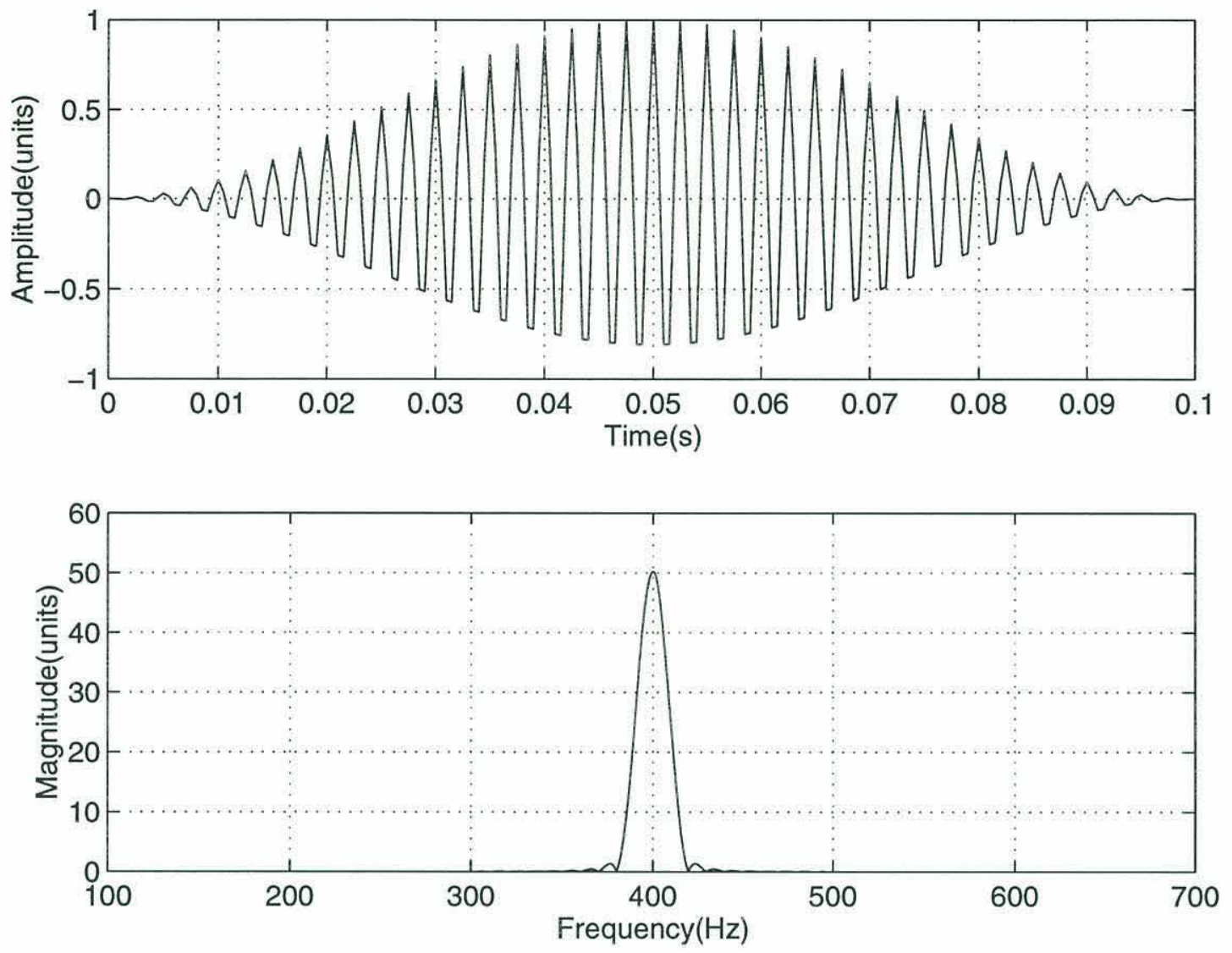

Figure 5-1: The source signal and its spectrum.

- Produce backscattering time series by Fourier synthesis of the scattered field in the full frequency spectrum.

Next we present an example of a simulated backscattering time series at one receiver. We will utilize the same free-space scenario as shown in Fig. 3-4. The background sound speed is chosen to be $1500 \mathrm{~m} / \mathrm{s}$, the density $1 \mathrm{~g} / \mathrm{cm}^{3}$, the source/receiver height $400 \mathrm{~m}$ and there is no attenuation. Figure 5-1 depicts the source signal that is going to be used in the simulation throughout this chapter and its spectrum. It is a Hanning-windowed $400 \mathrm{~Hz} \mathrm{CW}$ wave with a pulse duration of $0.1 \mathrm{~s}$. The sampling frequency is $2000 \mathrm{~Hz}$.

Assuming exponential correlation functions horizontally and vertically for a $7.5 \mathrm{~m}$ ( 2 wavelength) thick random layer with the horizontal correlation length $l_{x}=l_{y}=2 m$ 


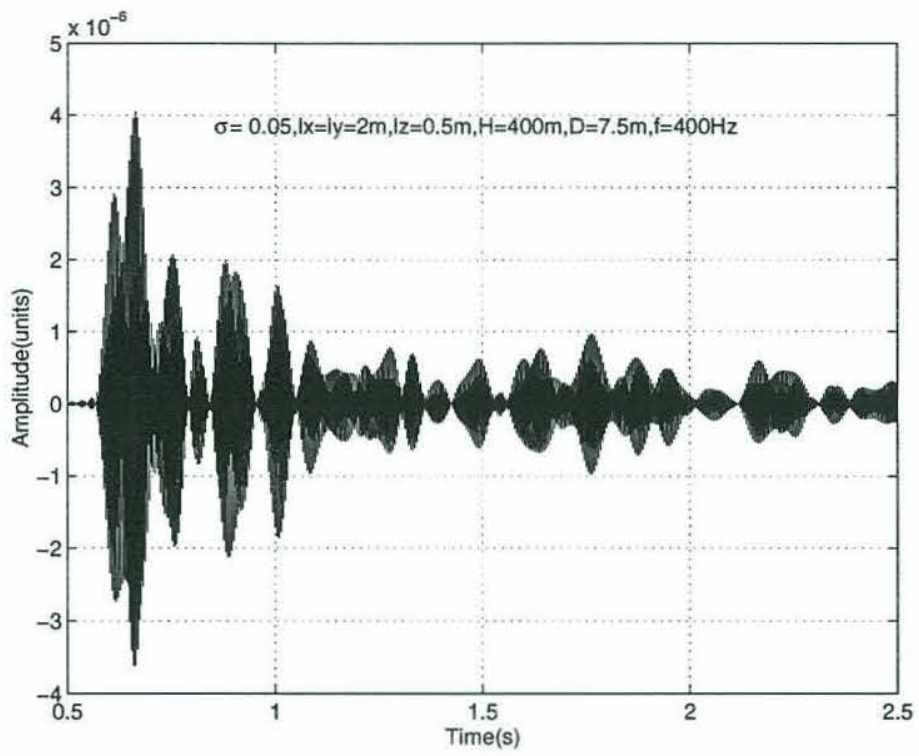

Figure 5-2: Simulated backscattered returns from an inhomogeneous layer. The correlation function of random sound speed variations within the layer are exponential both horizontally and vertically.

and vertical correlation length $l_{z}=0.5 \mathrm{~m}$, we can simulate backscattered returns recorded at the receiver as shown in Fig. 5-2. The single source/single receiver configuration is exploited and it is the setup for the rest of the chapter. The source/receiver height is fixed at $400 \mathrm{~m}$ from now on. Only sound speed variations will be modeled for the time being and the effect of density fluctuations will be examined in the last section of this chapter. Since the concentration of this thesis work is on mid- to low- grazing angle backscattering, we have tapered the random field close to normal incidence with half of a Hanning window. This causes an increasing amplitude at the beginning of the time series. The amplitude is then decaying with travel time increasing because of the spreading loss.

Simply by observing the simulated time series, it is almost impossible to tell if the numerical result is valid. Therefore, we have to find a way to prove that the generated backscattered returns make sense, which will be the task of the next section. 


\subsection{Comparisons with Analytic Solutions in the Free-Space Scenario}

With the quantum leap in the computational ability in recent years, numerical forward modeling has gained more and more popularity. However, there is always a question for this type of approach: how do we verify the numerical results? Usually there are two ways to check: the first and ideal scenario is to make comparisons of numerical results with appropriate analytic solutions, pending the availability of such analytic solutions; the second is to check if the model under development agrees with other numerical models or benchmark solutions, which is a legitimate verification except for some extreme cases. In the case that both of the above approaches cannot be carried out, the last but less convincing approach is to see if the numerical results make sense physically in solving some well-understood problems. Fortunately for this work, we have analytic solutions for monostatic backscattering from volumetric heterogeneities in the free-space case, which makes our life much easier. Formulations for such analytic solutions are given in Chapter 3. Subsequently, analytic results will be presented in this chapter without being reiterated in detail.

As we know in numerical modeling, one common practice is to discretize the continuous field, i.e., sampling. The final results would normally vary with the sampling rate unless adequate sampling of the field has been achieved. Therefore, it is critical to find out the sufficient sampling rate, thereby reaching a unique solution. An effective and widely used procedure is the convergence test. The idea is to increase the sampling rate until the end result approaches a constant value,i.e., converges. The lowest sampling rate for the result to reach the constant value is designated the sufficient sampling rate. Theoretically, convergence tests need to be performed for every problem. Figure 5-3 shows an example of a convergence test. The ensemble averages of backscattering intensity versus time are plotted for 50 realizations. Backscattering intensity here is defined as the square of the envelope of the backscattered returns. Meanwhile, from a statistics point of view, a single realization is not 


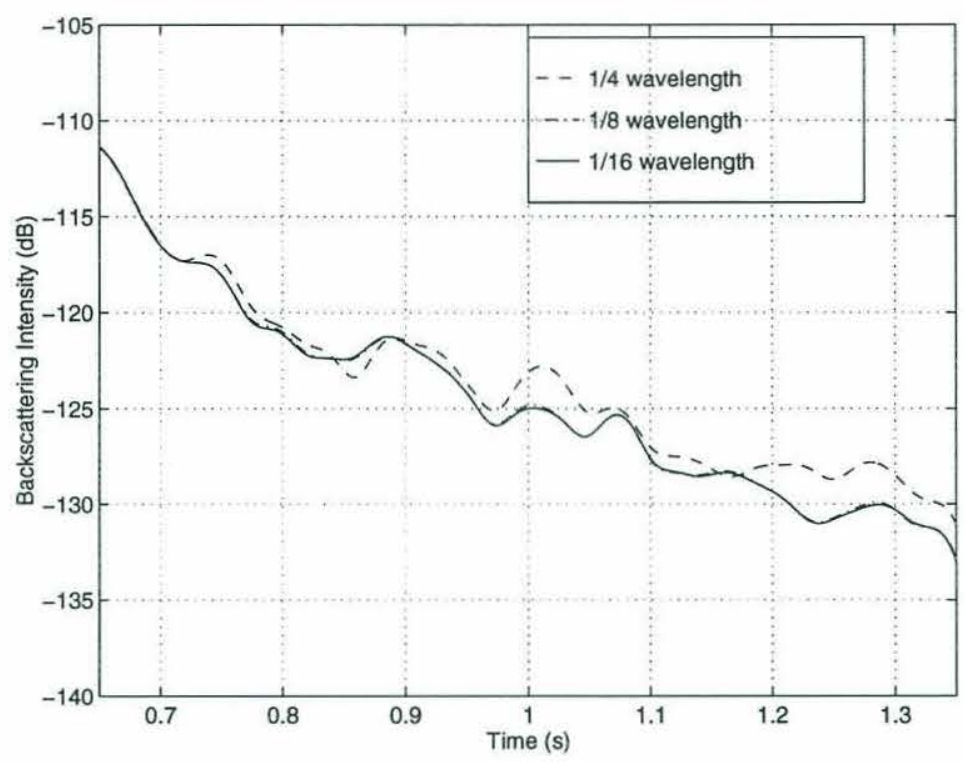

Figure 5-3: The convergence test for backscattering from 2-D sound speed variations with a power law distribution $\left(l_{x}=l_{y}=1 \mathrm{~m}\right)$.

sufficient to determine a statistical process. Consequently, ensemble averaging will instead be studied for the rest of the chapter. The result shown in Fig.5-3 is for backscattering from 2-D sound speed variations with a power law distribution. The horizontal correlation lengths $l_{x}$ and $l_{y}$ are $1 m$, respectively. The standard deviation of sound speed variations $\sigma$ is $5 \%$. From the figure, it can be seen that sampling interval of $1 / 8$ wavelength, i.e., a sampling rate of $8 k$ where $k$ is the wavenumber, is sufficient for the backscattering intensity to converge. Of course, the sufficient sampling rate will largely depend on what type of correlation function we have for the random field, or more specifically, the roll-off rate of the power spectrum versus wavenumber. Convergence tests have been done for Gaussian and exponential distributions as well, and the sampling interval of $1 / 8$ wavelength horizontally has been found to be adequate, although results are not shown here for the sake of brevity.

As mentioned in Chapters 2 and 3, for surface scattering or volume scattering from a thin layer, the quantity of scattering strength versus grazing angle can be used to characterize the scattering process. There is no angular ambiguity in this case even 
for a single source/single receiver configuration because the arrival time can be easily related to the scattering angle. The equation for the calculation of the backscattering strength is as follows

$$
B S S=10 \log _{10} \frac{I_{s} R^{2}}{I_{i n} A},
$$

where $I_{s}$ is the intensity of backscattered signals at the receiver, $I_{i n}$ is the intensity of the incident sound wave a unit distance away from the scattering area, $R$ is the distance from the source to the scatterer, and $A$ is the insonified area [52]. Following the same procedure as in Ref. [10], we have

$$
A=\pi c \tau R
$$

where $c$ is the sound speed and $\tau$ is the pulse duration. At the same time, $I_{s}$ and $I_{i n}$ can be estimated respectively by

$$
I_{s}=\frac{1}{\tau} \int_{t}^{t+\tau} y^{2}(T) d T,
$$

where $y(T)$ is the scattered return at the receiver and $t$ is the round trip travel time from the source/receiver to the scatterers and

$$
I_{\text {in }}=\frac{1}{\tau} \int_{t_{0}}^{t_{0}+\tau} S^{2}(t) d t,
$$

where $S(t)$ is the source signal. We will estimate the backscattering strength from the simulated backscattered returns and compare the results with analytic solutions obtained in Chapter 3.

We will categorize the comparisons into the 2-D isotropic case, the 2-D anisotropic case and the $3-\mathrm{D}$ vertically correlated case. 


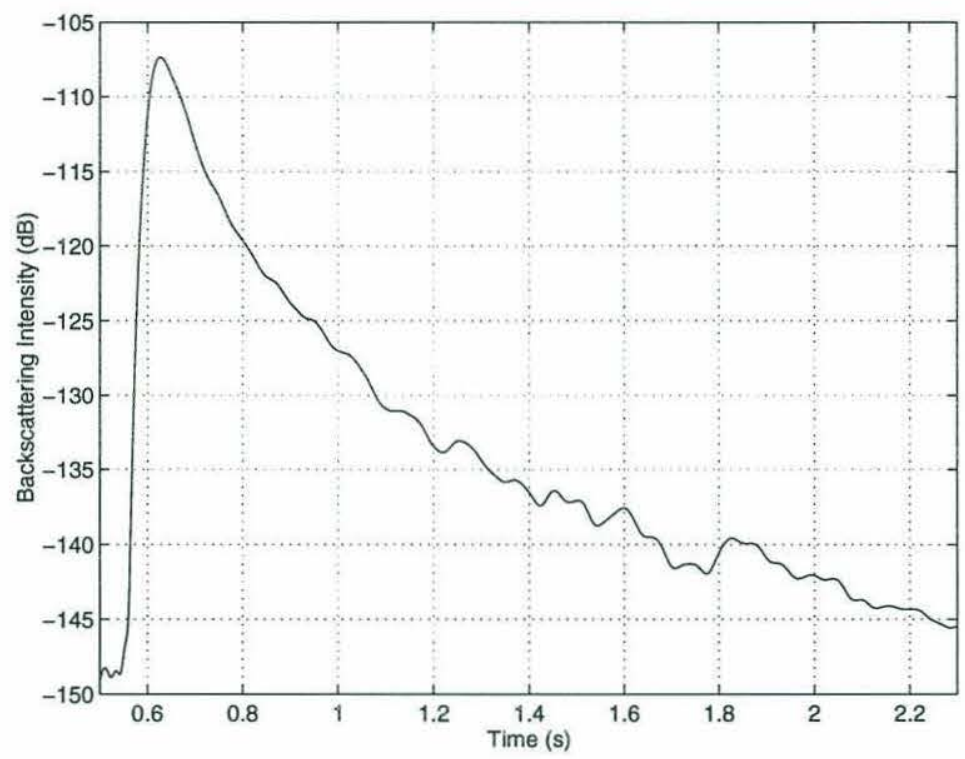

Figure 5-4: Backscattering from 2-D isotropic sound speed variations with a Gaussian correlation function, averaging over 50 realizations $\left(f=400 \mathrm{~Hz}, \mathrm{H}=400 \mathrm{~m}, l_{x}=l_{y}=\right.$ $1 m, \sigma=0.05)$

\subsubsection{2-D Isotropic Case}

First we will present an example of backscattering from random sound speed variations possessing a 2-D isotropic Gaussian correlation function. Figure 5-4 shows the ensemble average over 50 realizations of simulated backscattering intensities at one receiver. The source/receiver height is $400 \mathrm{~m}$. The frequency is $400 \mathrm{~Hz}$. The horizontal correlation lengths $l_{x}$ and $l_{y}$ are chosen to be $1 m$, respectively, and the standard deviation $\sigma$ is $5 \%$. The backscattering strength versus grazing angle can be estimated following the procedure presented in the beginning of this section. The comparison with the analytic solution can be seen in Fig. 5-5, in which the error bars show the standard deviation of the estimate. To our delight, the numerical solution and the analytic solution match very well.

Second is an example of backscattering from random sound speed variations with a 2-D isotropic exponential correlation function. Figure 5-6 shows the ensemble average over 50 realizations of simulated backscattering intensities at one receiver. All 


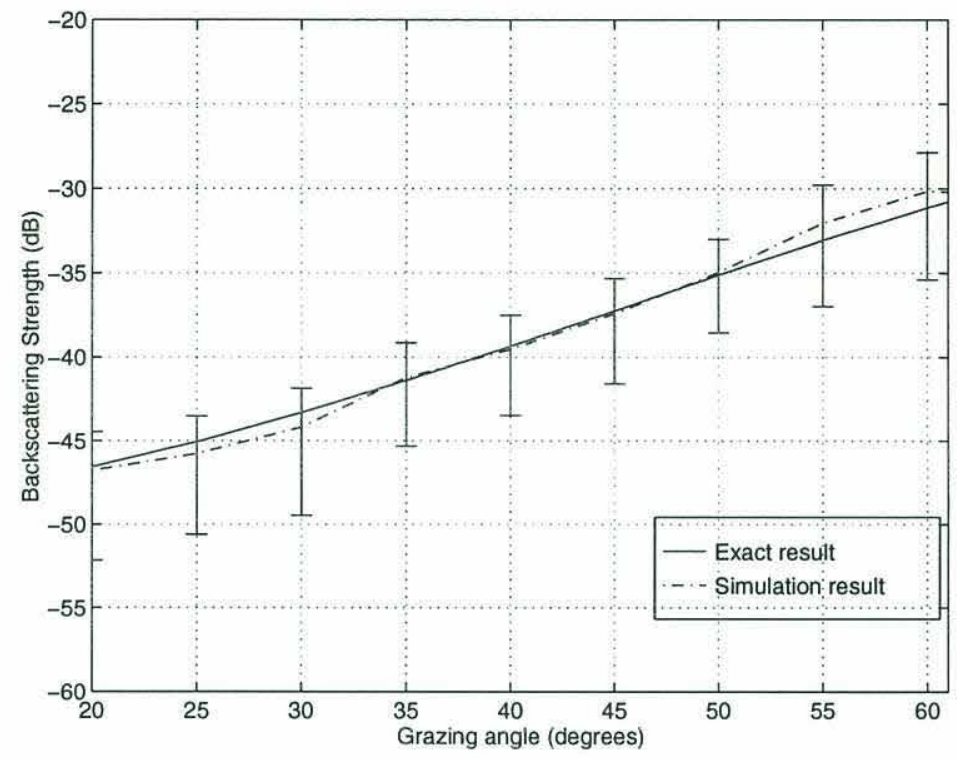

Figure 5-5: Comparison of the backscattering strength estimated from the numerically simulated backscattered returns with the analytic solution. Sound speed variations have a Gaussian correlation function. ( $f=400 \mathrm{~Hz}, \mathrm{H}=400 \mathrm{~m}, l_{x}=l_{y}=1 \mathrm{~m}, \sigma=$ $0.05)$

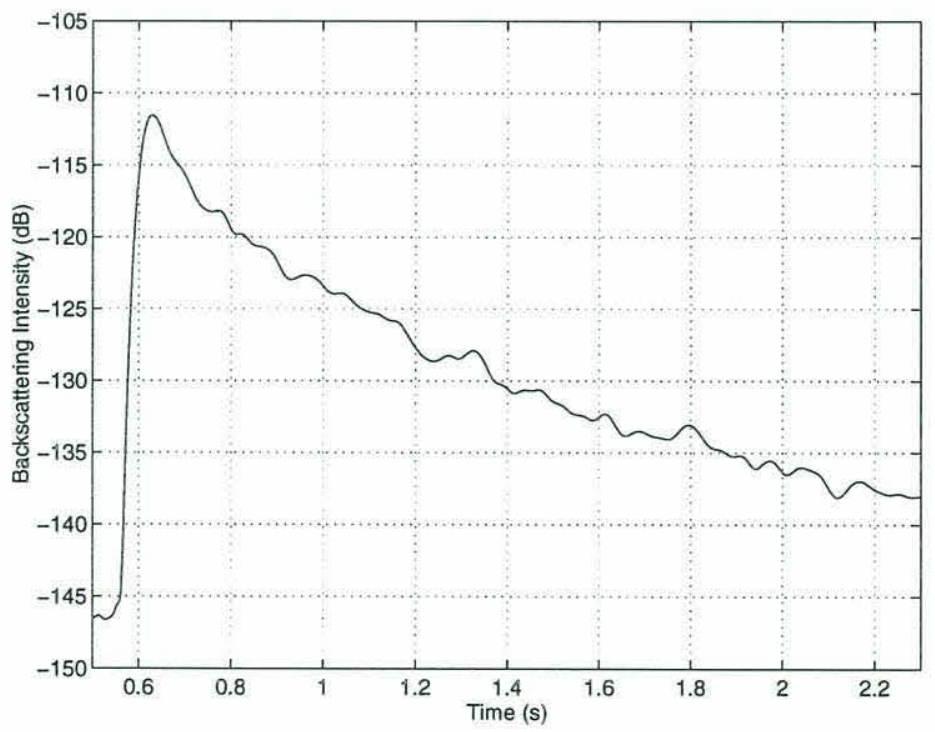

Figure 5-6: Backscattering from 2-D isotropic sound speed variations with an exponential correlation function, averaging over 50 realizations $(f=400 \mathrm{~Hz}, \mathrm{H}=$ $\left.400 m, l_{x}=l_{y}=0.84 m, \sigma=0.05\right)$ 


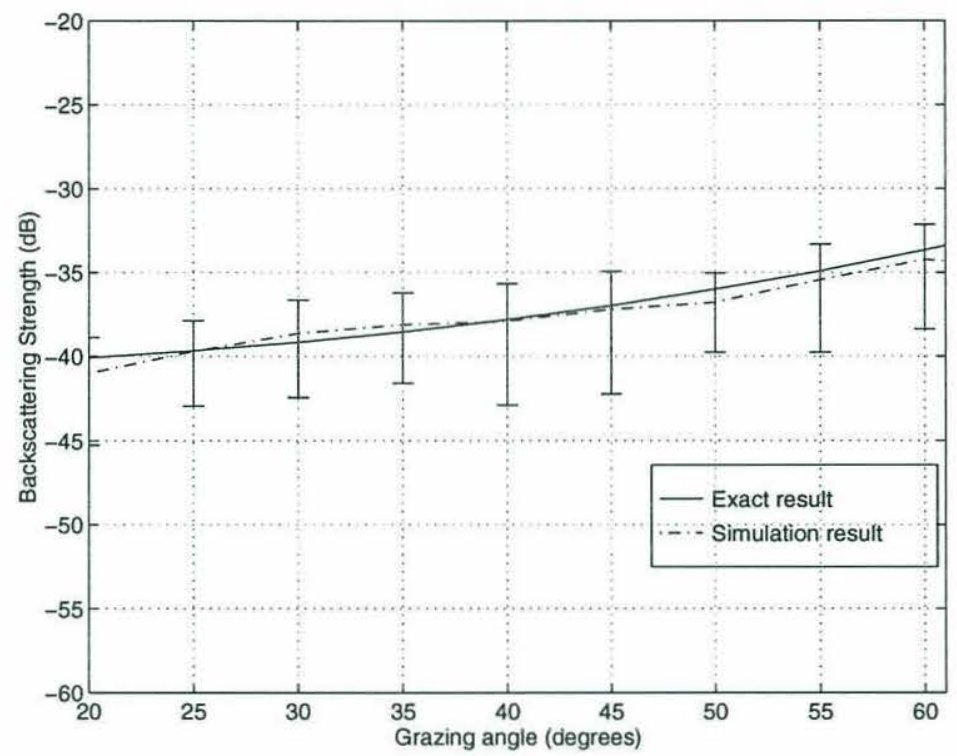

Figure 5-7: Comparison of the backscattering strength estimated from the numerically simulated backscattered returns with the analytic solution. Sound speed variations have an exponential correlation function. $\left(f=400 \mathrm{~Hz}, \mathrm{H}=400 \mathrm{~m}, l_{x}=l_{y}=\right.$ $0.84 m, \sigma=0.05)$

the parameters are the same as in the Gaussian correlation case except that the correlation lengths $l_{x}$ and $l_{y}$ are arbitrarily chosen to be $0.84 m$, respectively. Again, the backscattering strength versus grazing angle is estimated and the comparison with the analytic solution is plotted in Fig. 5-7. The matches between the two are excellent.

Third is an example of backscattering from random sound speed variations with a 2-D isotropic power law distribution. Figure 5-8 depicts the ensemble average over 50 realizations of simulated backscattering intensities at one receiver. All the parameters are the same as in the Gaussian correlation case and the horizontal correlation lengths are once again $1 m$ in both $x$ and $y$ directions. The order $\nu$ in the Von Karman function is chosen to be 1 so that the fractal dimension is 2 . This is the case for the anisotropic case in the next subsection as well. Figure 5-9 shows the comparison of the estimated backscattering strength with the analytic solution. The results again are excellent. 


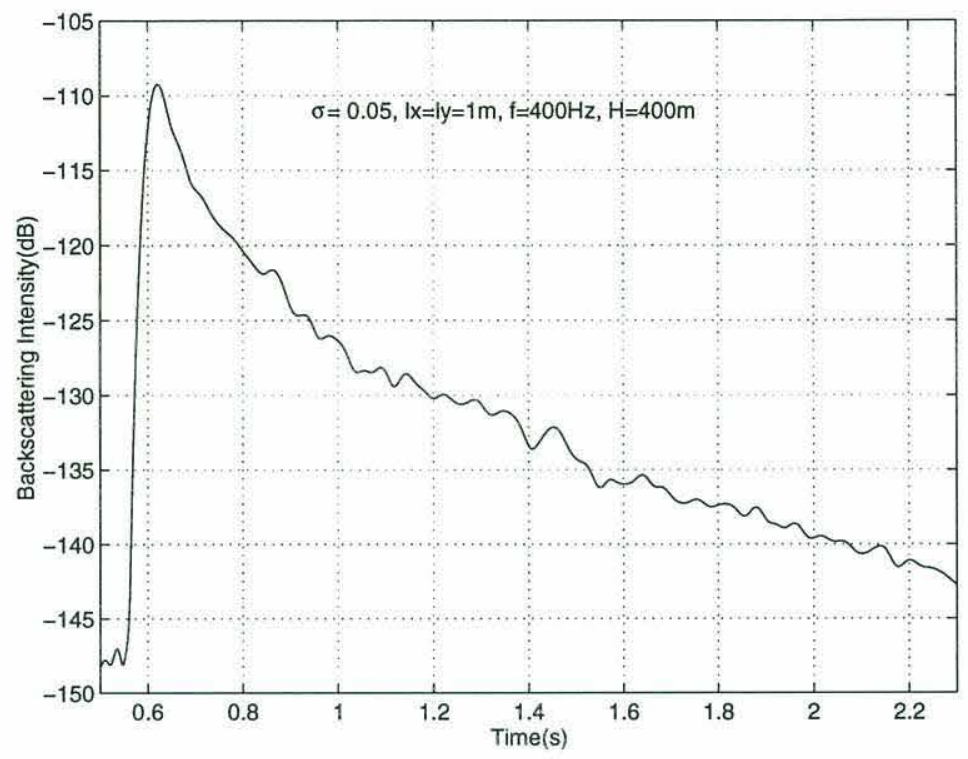

Figure 5-8: Backscattering from 2-D isotropic sound speed variations with a power law distribution, averaging over 50 realizations $\left(f=400 \mathrm{~Hz}, \mathrm{H}=400 \mathrm{~m}, l_{x}=l_{y}=\right.$ $1 m, \sigma=0.05, \nu=1$ )

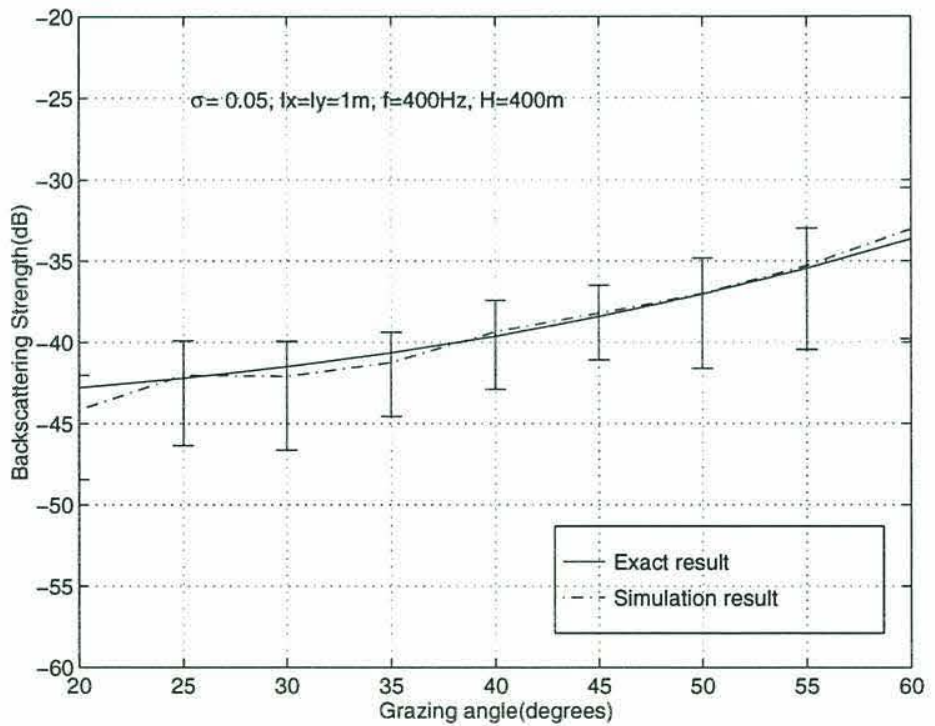

Figure 5-9: Comparison of the backscattering strength estimated from the numerical simulated backscattering returns with the analytic solution. Sound speed variations have a power law distribution. ( $f=400 \mathrm{~Hz}, H=400 \mathrm{~m}, l_{x}=l_{y}=1 \mathrm{~m}, \sigma=0.05, \nu=$ 1) 


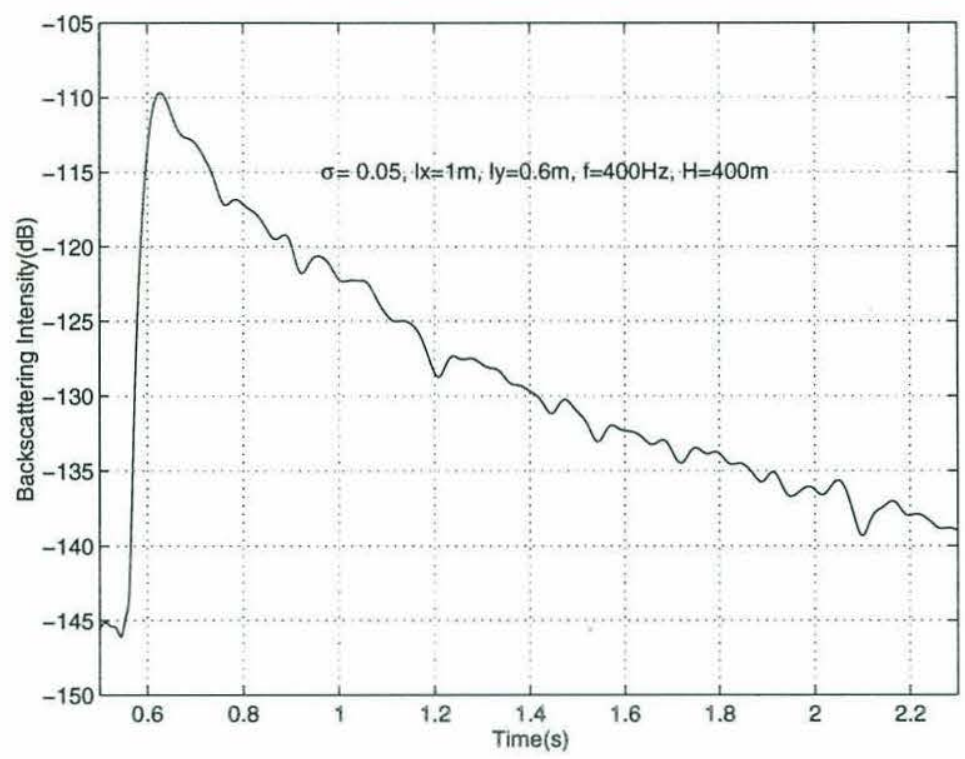

Figure 5-10: Backscattering from 2-D anisotropic sound speed variations with a Gaussian distribution, averaging over 50 realizations ( $f=400 \mathrm{~Hz}, \mathrm{H}=400 \mathrm{~m}, l_{x}=$ $\left.1 m, l_{y}=0.6 m, \sigma=0.05\right)$

\subsubsection{2-D Anisotropic Case}

The numerical model will be tested for 2-D anisotropic cases in this subsection. Again we will present examples for three different types of correlation functions.

Figure 5-10 demonstrates the anisotropic Gaussian case. The plotted backscattering intensity is the ensemble average of 50 realizations. All the parameters are the same as in the isotropic Gaussian case except that the correlation length in the $y$ direction $l_{y}$ has been changed to $0.6 \mathrm{~m}$. The comparison between numerical solutions and analytic solutions is shown in Fig. 5-11. For an anisotropic exponential distribution, the backscattering intensity versus arrival time is depicted in Fig. 5-12. The comparison of estimated backscattering strength with the analytic solution can be seen in Fig. 5-13. Except that the horizontal correlation length $l_{y}$ is changed to $2 m$, all other parameters are the same as in the isotropic Gaussian case. For an anisotropic power law distribution with correlation length $l_{x}=1 m, l_{y}=0.6 m$, and $\nu=1$, Fig. 5-14 shows the backscattering intensity and Fig. 5-15 the comparison 


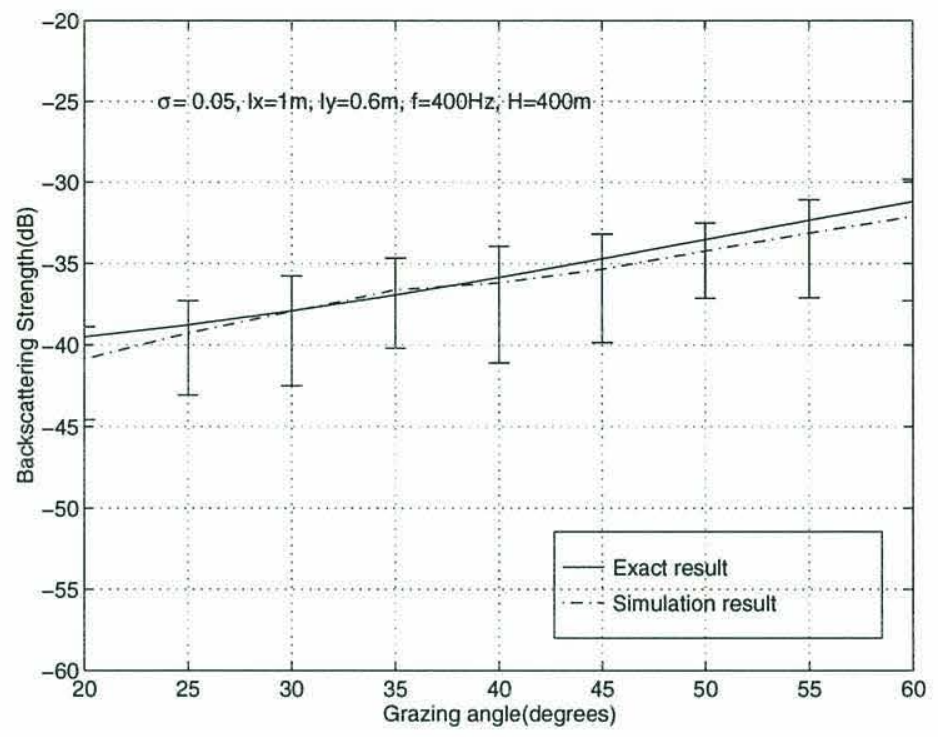

Figure 5-11: Comparison of the backscattering strength estimated from the numerically simulated backscattered returns with the analytic solution. Sound speed variations have a Gaussian distribution. $\left(f=400 \mathrm{~Hz}, H=400 \mathrm{~m}, l_{x}=1 \mathrm{~m}, l_{y}=0.6 m, \sigma=\right.$ $0.05)$

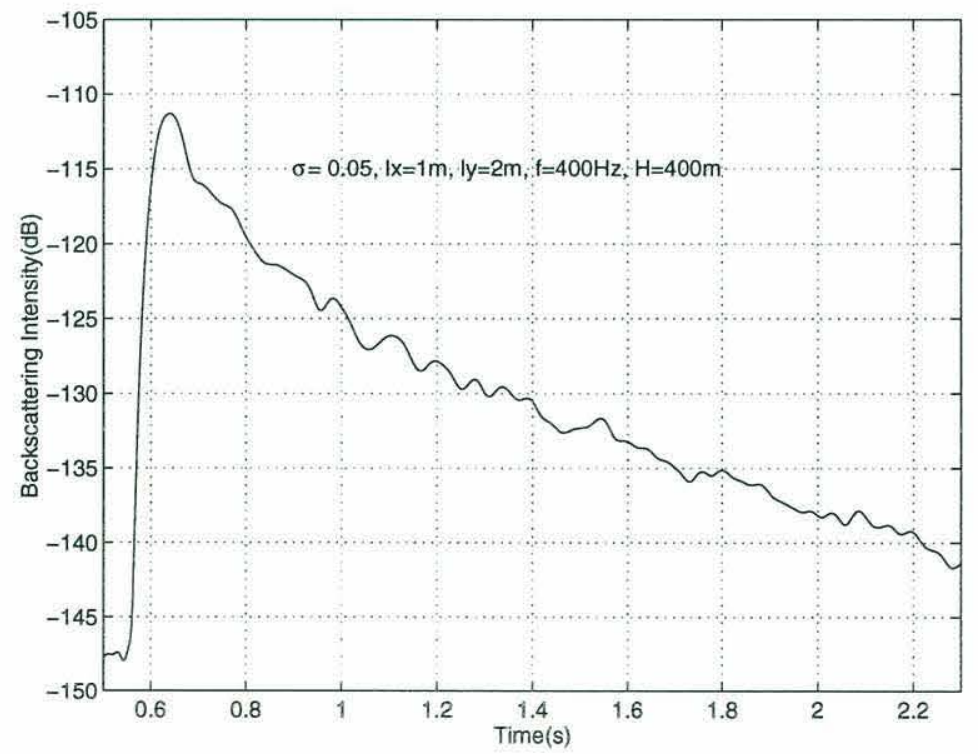

Figure 5-12: Backscattering from 2-D anisotropic sound speed variations with an exponential distribution, averaging over 50 realizations $\left(f=400 \mathrm{~Hz}, \mathrm{H}=400 \mathrm{~m}, l_{x}=\right.$ $\left.1 m, l_{y}=2 m, \sigma=0.05\right)$ 


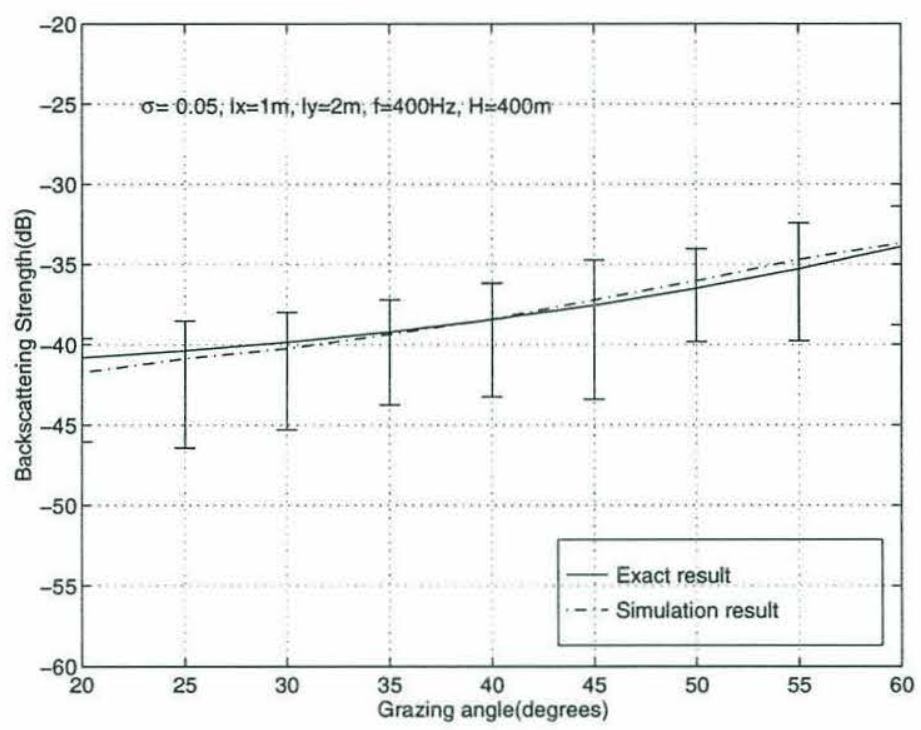

Figure 5-13: Comparison of the backscattering strength estimated from the numerical simulated backscattering returns with the analytic solution. Sound speed variations have an exponential distribution. $\left(f=400 \mathrm{~Hz}, \mathrm{H}=400 \mathrm{~m}, l_{x}=1 \mathrm{~m}, l_{y}=2 \mathrm{~m}, \sigma=\right.$ $0.05)$

with analytic solutions. It is evident that matches between the numerical results and the analytic solutions are excellent for all three of the above anisotropic cases.

For the sake of brevity, we give comparison of the backscattering strength versus grazing angle only for one set of parameters in each distribution. However, we have no doubt that the curves in Figs. 3-9, 3-10 and 3-11 can be reproduced by numerical simulation. Since the decay rate of the backscattering strength versus grazing angle is directly related to the decay rate of backscattering intensity versus time, we expect the backscattering intensity to have different decay rates versus time for different values of the parameters in the above three figures. Figure 5-16 gives one example in the Gaussian distribution case.

Through comparisons with analytic solutions in 2-D isotropic and anisotropic situations, the monostatic backscattering model has been proved to be successful in simulating backscattered returns resulting from 2-D random sound speed variations. 


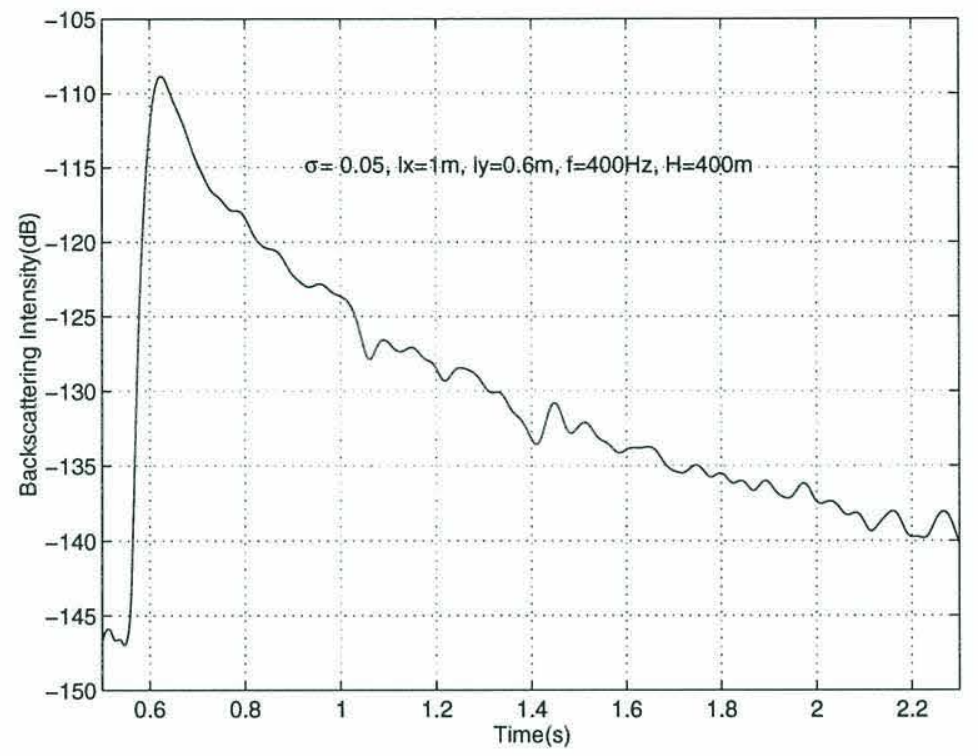

Figure 5-14: Backscattering from 2-D anisotropic sound speed variations with a power law type of distribution, averaging over 50 realizations $\left(f=400 \mathrm{~Hz}, \mathrm{H}=400 \mathrm{~m}, l_{x}=\right.$ $1 m, l_{y}=0.6 m, \sigma=0.05, \nu=1$ )

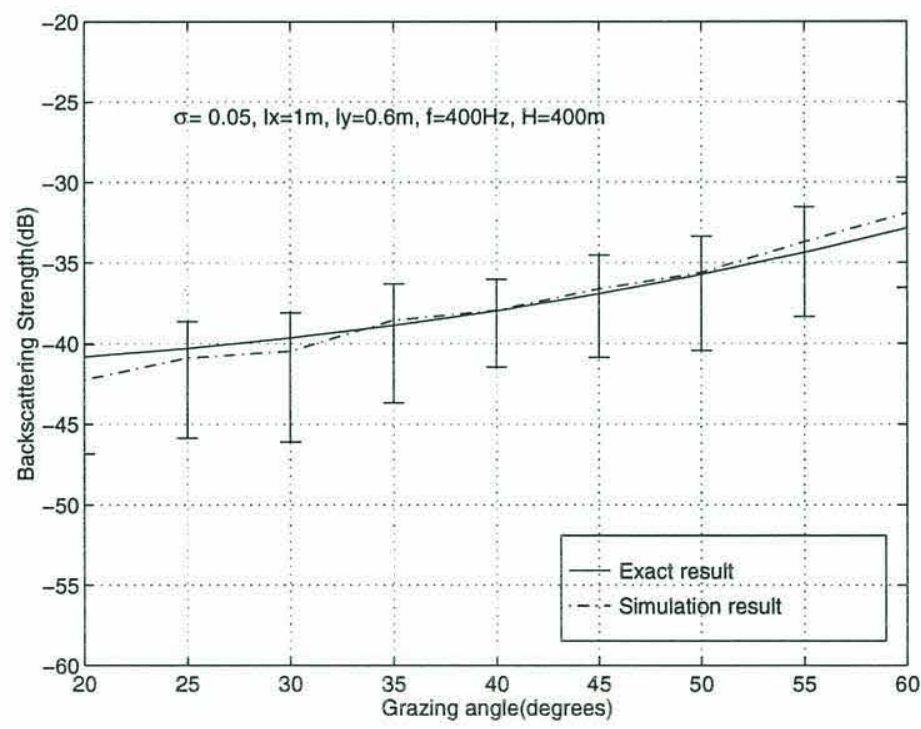

Figure 5-15: Comparison of the backscattering strength estimated from the numerically simulated backscattered returns with the analytic solution. Sound speed variations have a power law distribution. $\left(f=400 \mathrm{~Hz}, \mathrm{H}=400 \mathrm{~m}, l_{x}=1 \mathrm{~m}, l_{y}=0.6 \mathrm{~m}, \sigma=\right.$ $0.05, \nu=1)$ 


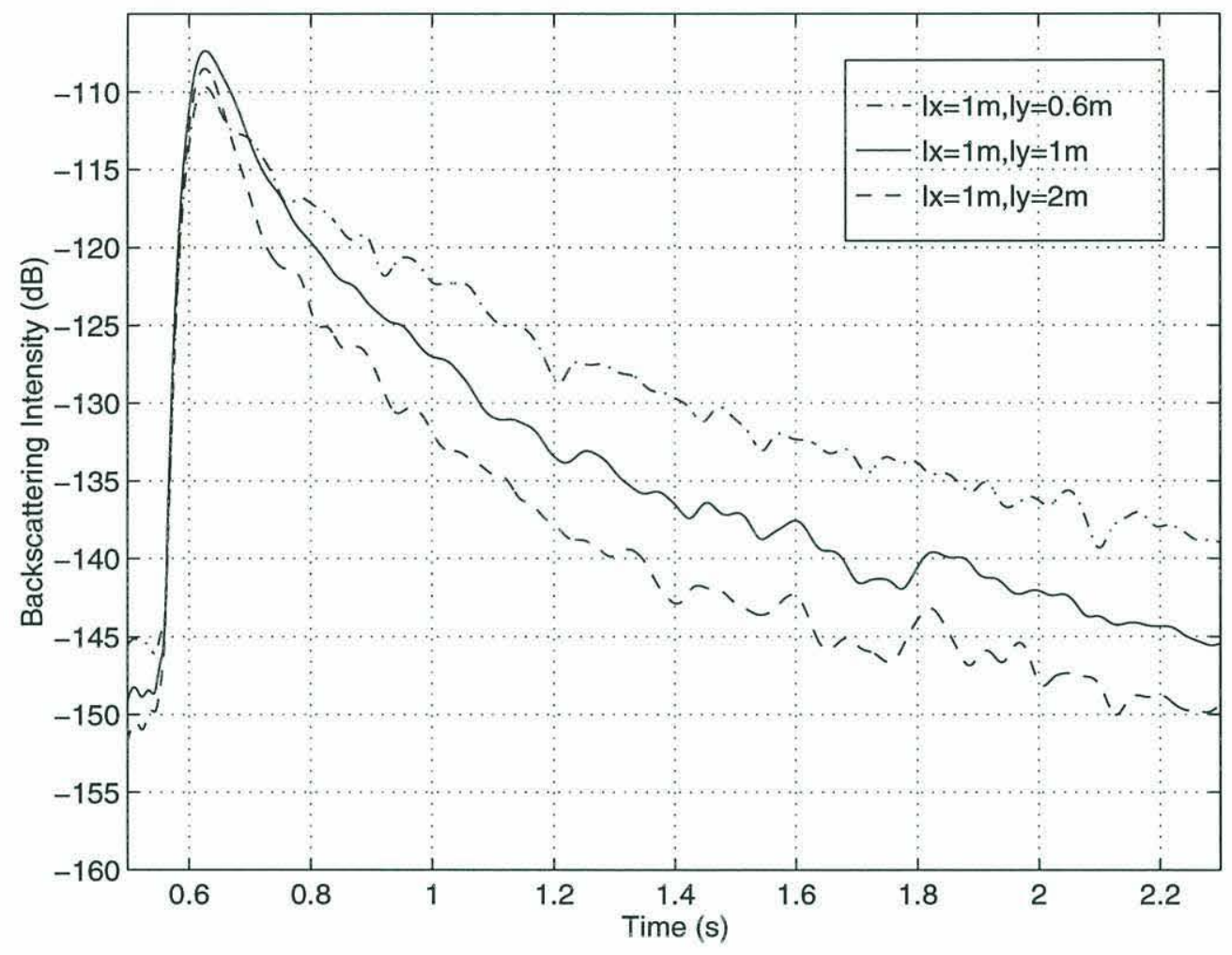

Figure 5-16: Backscattering intensities for simulated backscattered returns due to sound speed variations with a Gaussian distribution, averaging over 50 realizations $(f=400 \mathrm{~Hz}, H=400 \mathrm{~m}, \sigma=0.05)$. 
The good fit between the numerical results and analytic solutions also implies that the $1 / 8$ wavelength sampling interval is sufficient for all the above distributions.

\subsubsection{3-D Vertically Correlated Case}

Three-dimensional volume scattering with vertical correlation is more complicated than the above 2-D cases. On the one hand, the existence of physical boundaries, i.e., the layer interfaces, violates the stationarity assumption of the random field, which causes the edge effect that does not arise in the numerical solution. On the other hand, arrival time can no longer be translated into scattering angle accurately. Returns from scatterers located at different depths can have the same arrival time, which brings in angular ambiguities for the estimated backscattering strength in a single source/single receiver configuration.

In this section, our focus is on thin scattering layers. As long as the layer thickness is small, the single source/single receiver configuration is still capable of providing a good estimate of the backscattering strength versus grazing angle. However, in Chapter 2, an assumption is made in obtaining the analytic solution that the dimension of the scattering volume be much larger than the correlation length. This would conflict with the small layer thickness requirement above. A compromise has to be reached in choosing the layer thickness.

Again, we need a convergence test to determine the sufficient sampling rate in the vertical direction. In Fig. 5-17, we choose a $7.5 \mathrm{~m}$ thick random layer with exponential correlation function horizontally and vertically. The correlation lengths are $l_{x}=l_{y}=2 \mathrm{~m}$ and $l_{z}=0.5 \mathrm{~m}$. The frequency is $400 \mathrm{~Hz}$ and the source/receiver height is $400 \mathrm{~m}$. It can been seen that the curve with $1 / 10$ wavelength as sampling interval in the $z$ direction is very close to that with $1 / 20$ wavelength. Disregarding the beginning part which corresponds to high grazing angles, there are only up to $0.3 d B$ discrepancies along the curve which can be considered acceptable. Therefore, 1/10 


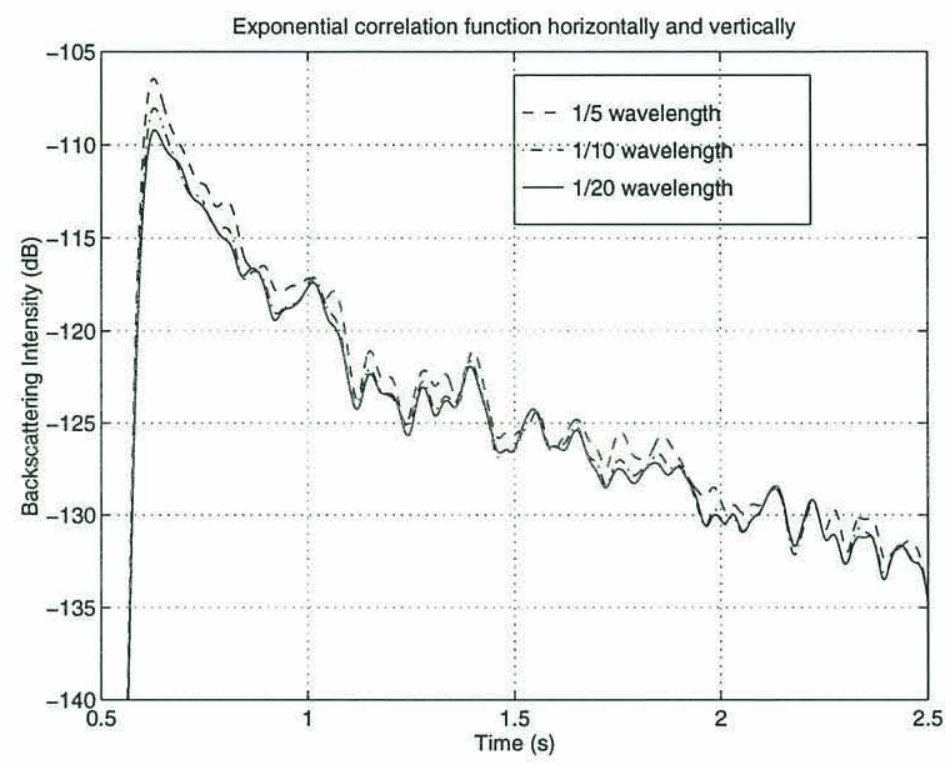

Figure 5-17: The convergence test for backscattering from 3-D random sound speed variations with exponential distributions horizontally and vertically $\left(\sigma=0.05, l_{x}=\right.$ $l_{y}=2 m, l_{z}=0.5 m, f=400 \mathrm{~Hz}, \mathrm{H}=400 \mathrm{~m}, \mathrm{D}=7.5 \mathrm{~m}$ )

wavelength is regarded as a sufficient sampling interval in the vertical direction.

Following the same procedure as in the 2-D cases, the backscattering strength can be estimated from simulated backscattered returns. The comparison with the analytic solution is shown in Fig. 5-18. The match between the two curves is not as good as that in the 2-D cases. However, except for regions close to 55 degree grazing angle, where the difference is about $2 d B$, the two curves are close to each other. Bear in mind that the analytic solution is obtained under the assumption that the dimension of the scattering volume is much larger than the correlation length so that the boundary effect can be ignored, while the numerical solution does include the boundary effect. That could well be the reason why such disagreement exists. Anyway, the fit can be regarded as satisfactory since the numerical solution predicts the trend of the backscattering strength versus grazing angle reasonably well and the level closely.

In conclusion, the numerical solution obtained from the monostatic backscattering 


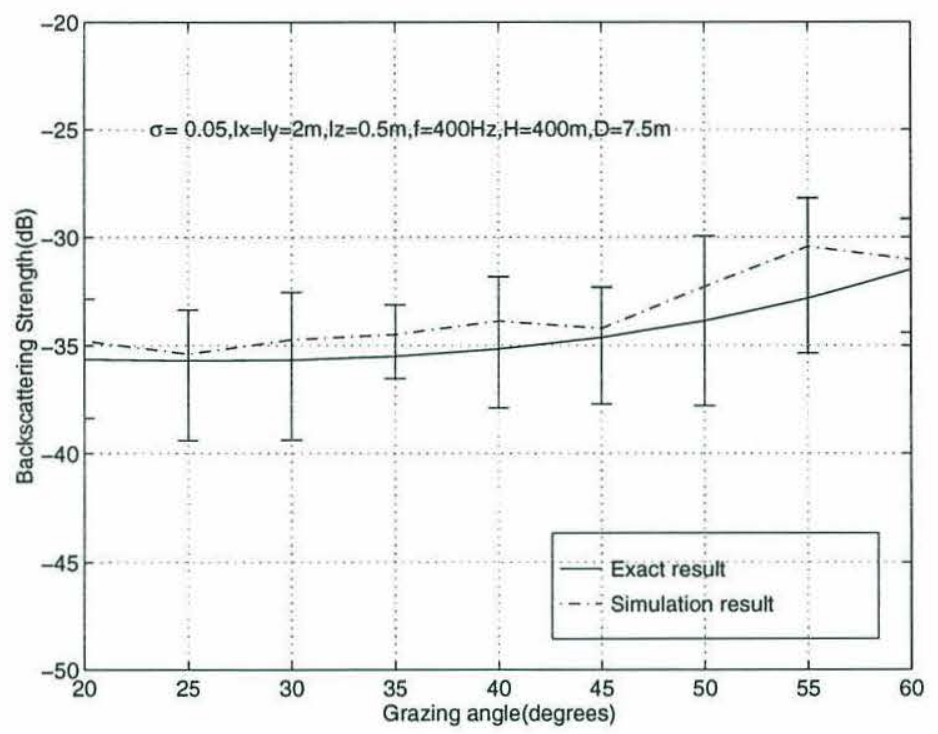

Figure 5-18: Comparison of the estimated backscattering strength from numerically simulated backscattered returns with the analytic solution. Sound speed variations are exponentially correlated horizontally and vertically. ( $\sigma=0.05, l_{x}=l_{y}=2 m, l_{z}=$ $0.5 m, f=400 \mathrm{~Hz}, H=400 \mathrm{~m}, \mathrm{D}=7.5 \mathrm{~m}$ )

model has been found to be in good agreement with the analytic solution. It gives us confidence that numerical errors are under control and the model prediction is valid, which sets the stage for the model/data comparison in the next chapter.

\subsection{The Effect of Density Variations}

As pointed out in Chapter 3, the density variations act like a dipole source with its major axis in the backscattering direction. Therefore, we would expect total backscattering to be enhanced. To confirm this, the following procedures are to be taken: First, backscattering returns from 2-D random sound speed variations only will be simulated. Sound speed variations are assigned a power law distribution with correlation lengths $l_{x}=l_{y}=1 \mathrm{~m}$. The standard deviation is $5 \%$. The frequency is $400 \mathrm{~Hz}$ and the source/receiver height is $400 \mathrm{~m}$. The backscattering intensity can then be obtained and the backscattering strength estimated. Next, we add density varia- 


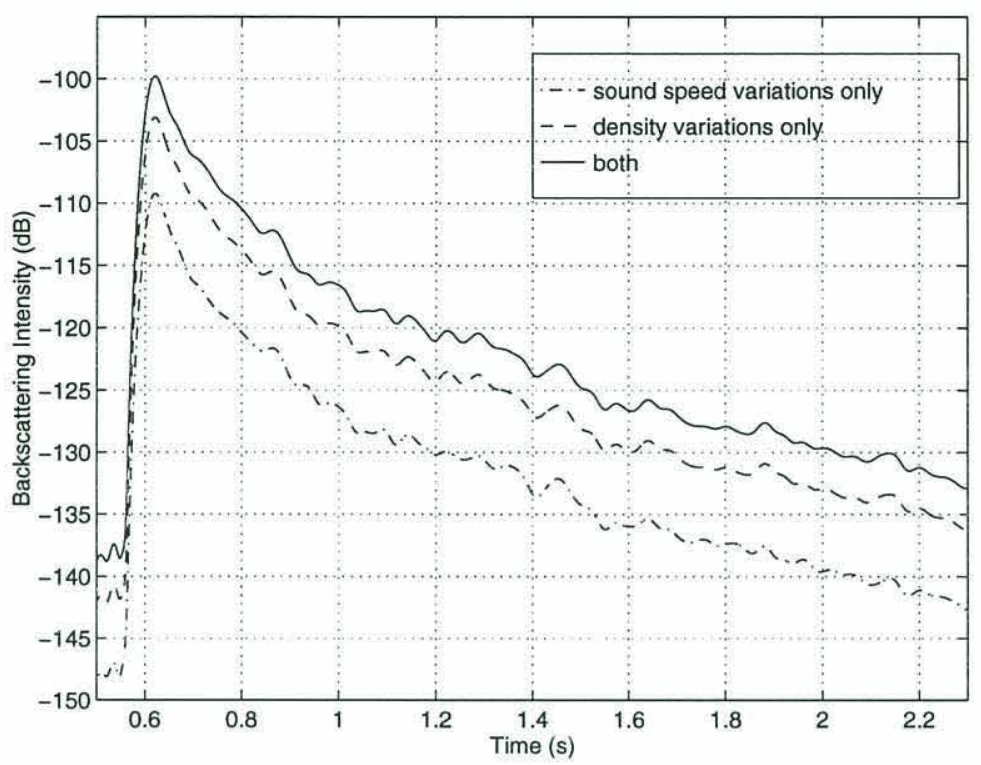

Figure 5-19: Backscattering intensities for simulated backscattered returns due to 2-D random sound speed variations only(dash-dotted line) and density variations only(dash line) and both(solid line). Sound speed variations have a power law distribution. $\left(\sigma=0.05, l_{x}=l_{y}=1 \mathrm{~m}, \beta=2, f=400 \mathrm{~Hz}, \mathrm{H}=400 \mathrm{~m}\right)$

tions to the picture. The ratio between density variations and sound speed variations, i.e., $\beta$ is chosen to be 2 according to Hines' empirical results [14]. The backscattering intensity is recalculated and the backscattering strength again estimated.

Backscattering intensities with sound speed variations only, with density variations only and with both variations are plotted together in Fig. 5-19 and the corresponding backscattering strengths in Fig. 5-20. The backscattering strength with density variations only is $7 d B$ higher than that with sound speed variations only, which shows that density variations are the more dominant contributors to backscattering. An interesting phenomenon is that the two curves are parallel to each other. In other words, the angular dependence of the backscattering strength has not changed with the addition of density variations. One possible explanation is that the major axis of the virtual dipole-like source caused by density variations always lies in the backscattering direction. While density variations and sound speed variations are fully correlated in the meantime, the dipole-like source will behave the same as the monopole-like source caused by sound speed variations in the backscattered direction. 


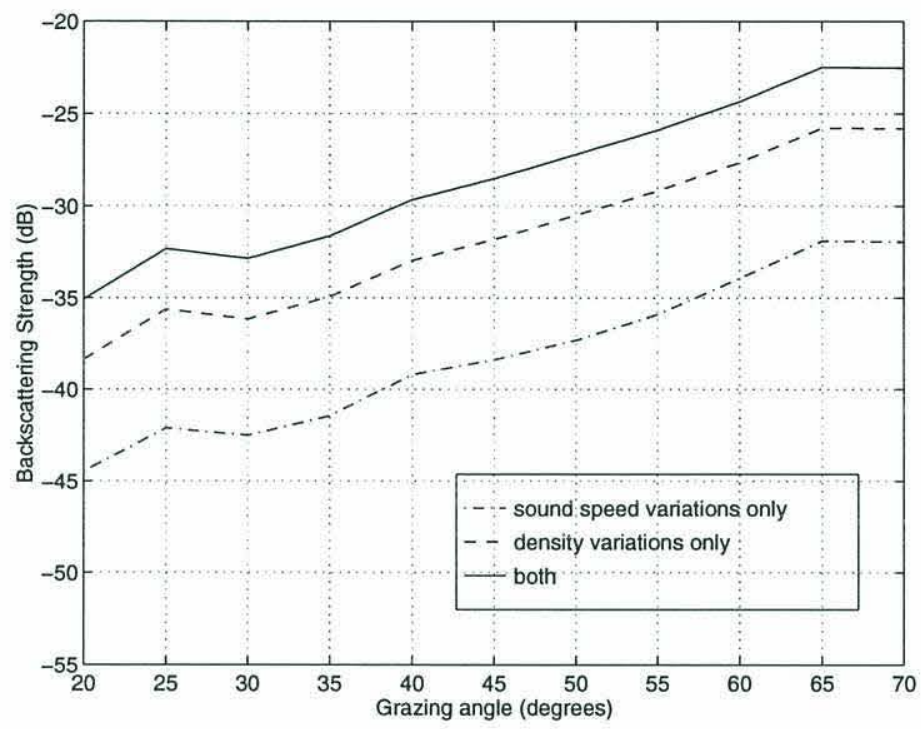

Figure 5-20: Backscattering strengths for simulated backscattered returns due to 2-D random sound speed variations only(dash-dotted line) and density variations only(dash line) and both(solid line). Sound speed variations have a power law distribution. $\left(\sigma=0.05, l_{x}=l_{y}=1 m, \beta=2, f=400 H z, H=400 m\right)$

All in all, the above examples have clearly demonstrated the importance of including density variations in backscattering modeling. Since density variations will change only the level but not the angular dependence of the backscattering strength, the conclusions that we have reached through the study of sound speed variations only will still be valid. 


\section{Chapter 6}

\section{Comparison of Model Results and}

\section{ARSRP Backscattering Data}

A primary goal of this work is to develop a volume backscattering model that can account for the backscattering phenomena observed in the ARSRP sediment experiment. Throughout Chapter 2, 3, and 4, the accumulating efforts have clearly paved the way for the realization of a backscattering model, which is capable of incorporating contributions due to both sound speed and density variations. In the meanwhile, by comparing to analytic solutions in free-space scenarios, the reliability of the model has been demonstrated in the previous chapter. Nevertheless, a more important but challenging task is to evaluate the capability of the model in predicting the experimental results, which is the concentration of this chapter.

As discussed earlier, an ideal and straightforward way to compare the model and backscattering data due to volume scattering from a thick layer of sediment inhomogeneities is through the scattering time series. However, in the ARSRP experiment scenario, the wide bandwidth of the source signal, $400 \mathrm{~Hz}$, together with the large layer thickness, $20-30 m$ (has to be sampled at every $1 / 10$ th wavelength vertically in order to guarantee convergence) make the simulation of the scattering time series extremely computationally intensive. It would be unrealistic in this situation to run the model many times so as to achieve a good fit with the data. Therefore, an al- 
ternative scheme for comparison is chosen, which is to compare the backscattering strength versus grazing angle between model and data for selected frequencies across the band. One might recall that the single source/single receiver configuration cannot distinguish scattered returns from different directions but with the same arrival time, thereby resulting in angular ambiguities of the backscattering strength. Fortunately for us, this drawback can be overcome thanks to the vertical line array and beamforming technique. Besides, as long as the model and data are processed by the same means, the results of comparisons will not be affected.

This chapter begins by reviewing the experimental setup. All the information about the backscattering data collection is provided in the first section. The ARSRP data processing will then be briefly discussed in the second section. In the third section, the focus will be on the estimation of the backscattering strength from the beamformed backscattered returns. Details will also be given on how to choose the model parameters since they are closely related to the above estimation. The comparison between model results and the ARSRP backscattering data is the theme of the final section.

\subsection{Experiment Description}

On July 24-26, 1993, as part of the Acoustic Reverberation Special Research Program (ARSRP), a bottom backscattering experiment was conducted over the site A sediment pond located at $26^{\circ} 11^{\prime} \mathrm{N}, 46^{\circ} 09^{\prime} \mathrm{W}$ in the vicinity of the Mid-Atlantic Ridge [76].

The water depth was about 4400 meters for most of the sediment pond area and the sound speed profile was a typical linear deep ocean profile obtained from XBT measurements. The gradient was $0.0154 / \mathrm{s}$ for the water column close to the seafloor. The sound speed at the water-sediment interface was extrapolated to be $1530 \mathrm{~m} / \mathrm{s}$. 
The oval-shaped sediment pond was approximately $15 \mathrm{~km} \times 20 \mathrm{~km}$ in size. The thickness of the sediment cover varied from almost no cover at the edge of the pond to about 430 meters in the middle of the pond. For areas where the backscattered data were collected, the sediment was at least $100 \mathrm{~m}$ thick. The top layer beneath the water-sediment interface was made of calcareous mud with a sound speed of $1510 \mathrm{~m} / \mathrm{s}$ according to core data [77]. No direct measurements of the attenuation coefficient or the sound speed gradient were available in the sediment area where the experiment was conducted. A deep towed high-frequency chirp sonar and in situ optical survey revealed that the seafloor was virtually flat with rms roughness less than $10 \mathrm{~cm}$ [78]. A rough basalt basement was beneath the sediment layer.

Figure 6-1 shows the bathymetry of the sediment pond and the ship track along which the experiment was carried out. For the results presented in this chapter, we will only analyze the data collected along the straight line shown in the figure. Figure 6-2 depicts the experimental scenario. The acoustic system was deployed at the west side of the pond and recovered in the middle after being towed across the entire sediment pond.

The acoustic source used in the experiment is part of the Deep Towed Acoustics/Geophysics System (DTAGS) and was developed by the Naval Research Laboratory - Stennis Space Center[79]. The source signal is a linear frequency-modulated (LFM) upchirp signal in the frequency range $250-650 \mathrm{~Hz}$. The designed source level is $200 \mathrm{~dB}$ re $1 \mu \mathrm{Pa} @ 1 \mathrm{~m}$. The duration of the source signal is $0.125 \mathrm{~s}$. The receiver is a 24 element vertical line array(VLA) hung beneath the source, with a spacing between adjacent hydrophone elements of $2 \mathrm{~m}$. The cable connecting the source and the top hydrophone is $57.5 \mathrm{~m}$ long. A weight is attached to the end of the array to keep it close to a vertical configuration. Figure 6-3 shows the geometry of the acoustic transmitting and receiving system. The source signal is transmitted once every 32 $\mathrm{s}$. For each ping, the hydrophones record $4.5 \mathrm{~s}$ of waveforms starting $0.1 \mathrm{~s}$ before the source trigger. The sampling interval is $0.432 \mathrm{~ms}$. More than 5000 pings were 


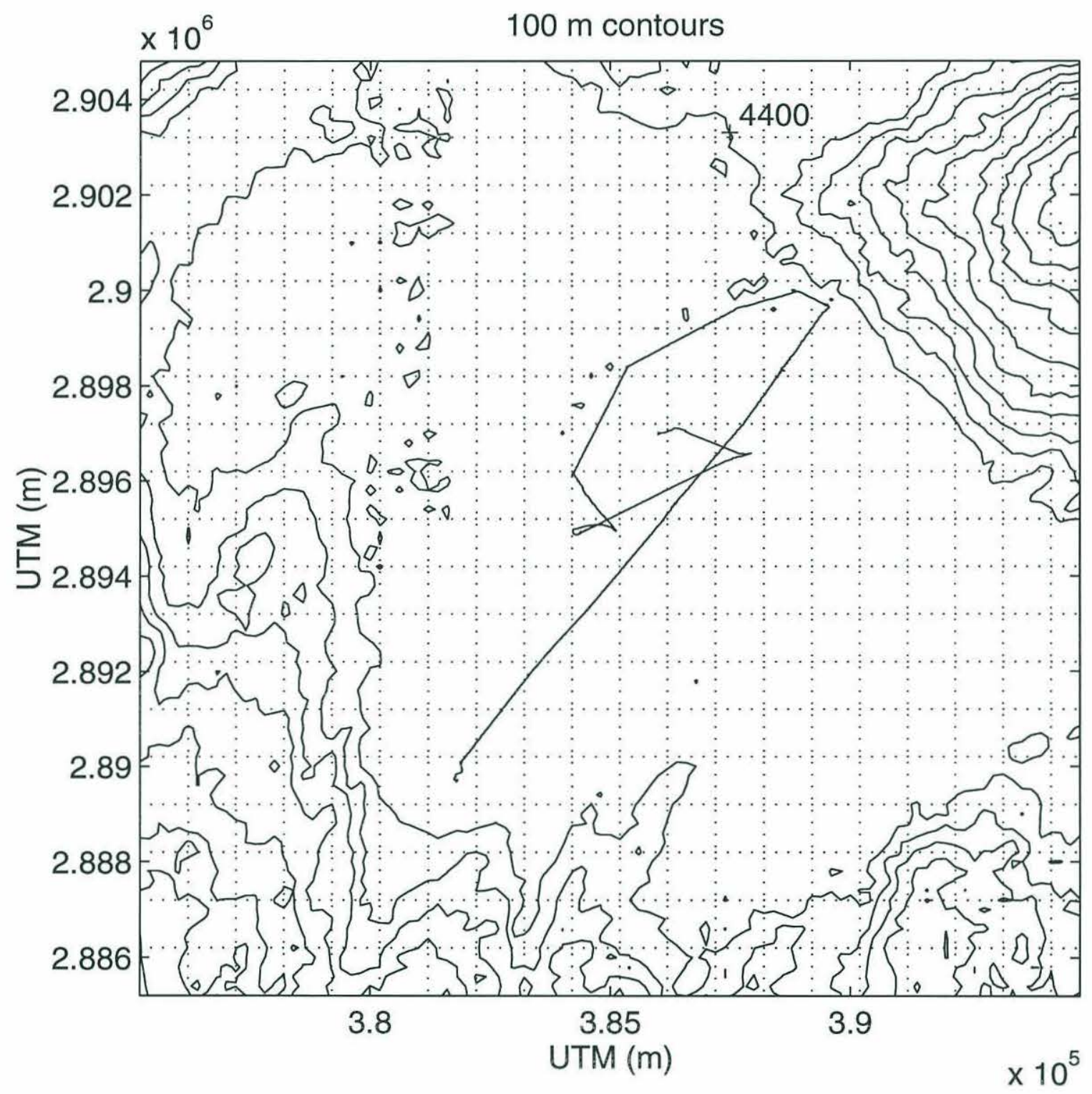

Figure 6-1: Bathymetry and ship track in the ARSRP Site A sediment pond. 
ARSRP Sediment Scatter Experiment

R/V Knorr, July 24 - 26, 1993

$26^{\circ} 11^{\prime} \mathrm{N} 46^{\circ} 09^{\prime} \mathrm{W}$

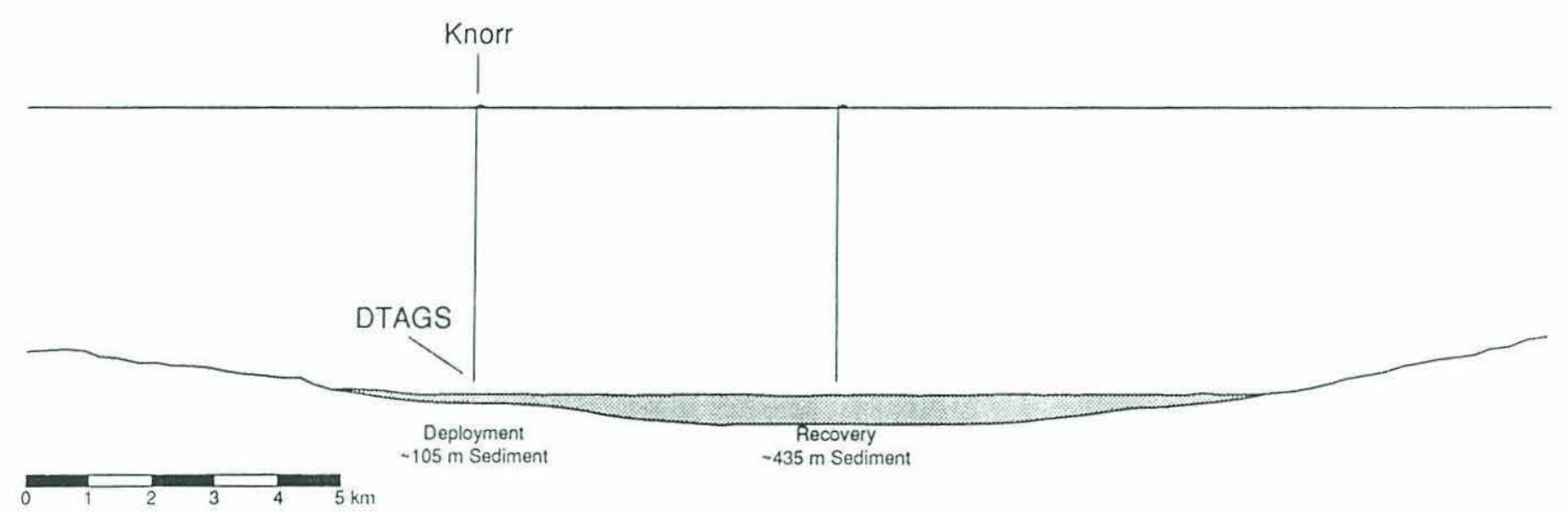

Figure 6-2: The experimental scenario. 


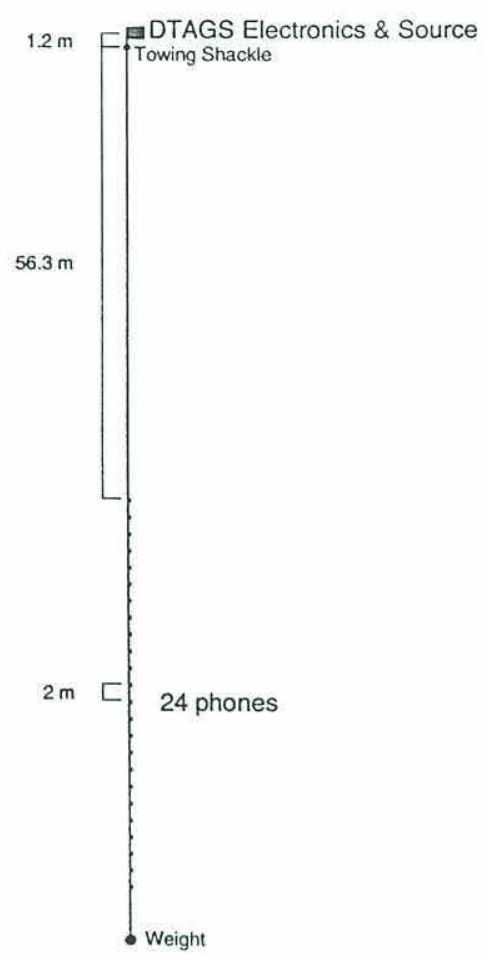

Figure 6-3: The DTAGS source and receiving array geometry.

transmitted throughout the experiment. Figure 6-4 shows the received signal and its spectrum at one hydrophone for one ping.

During the experiment, the DTAGS source was towed at a depth of about 200400 meters above the seafloor, while the ship used the high-resolution (P-code) GPS dynamic positioning to maintain a constant speed over ground (SOG) of $0.5 \mathrm{knot}$, with several stops to ensure that the array returned to nearly vertical alignment. The actual array shape is estimated by using the direct and bottom reflected arrival times on each hydrophone. The details of the array element localization method was given in [10]. Figure 6-5 shows the estimated array configuration for one location. It can be noticed that the array is roughly vertical. Generally it is found that the array tilt angle is within 5 degrees of vertical. 

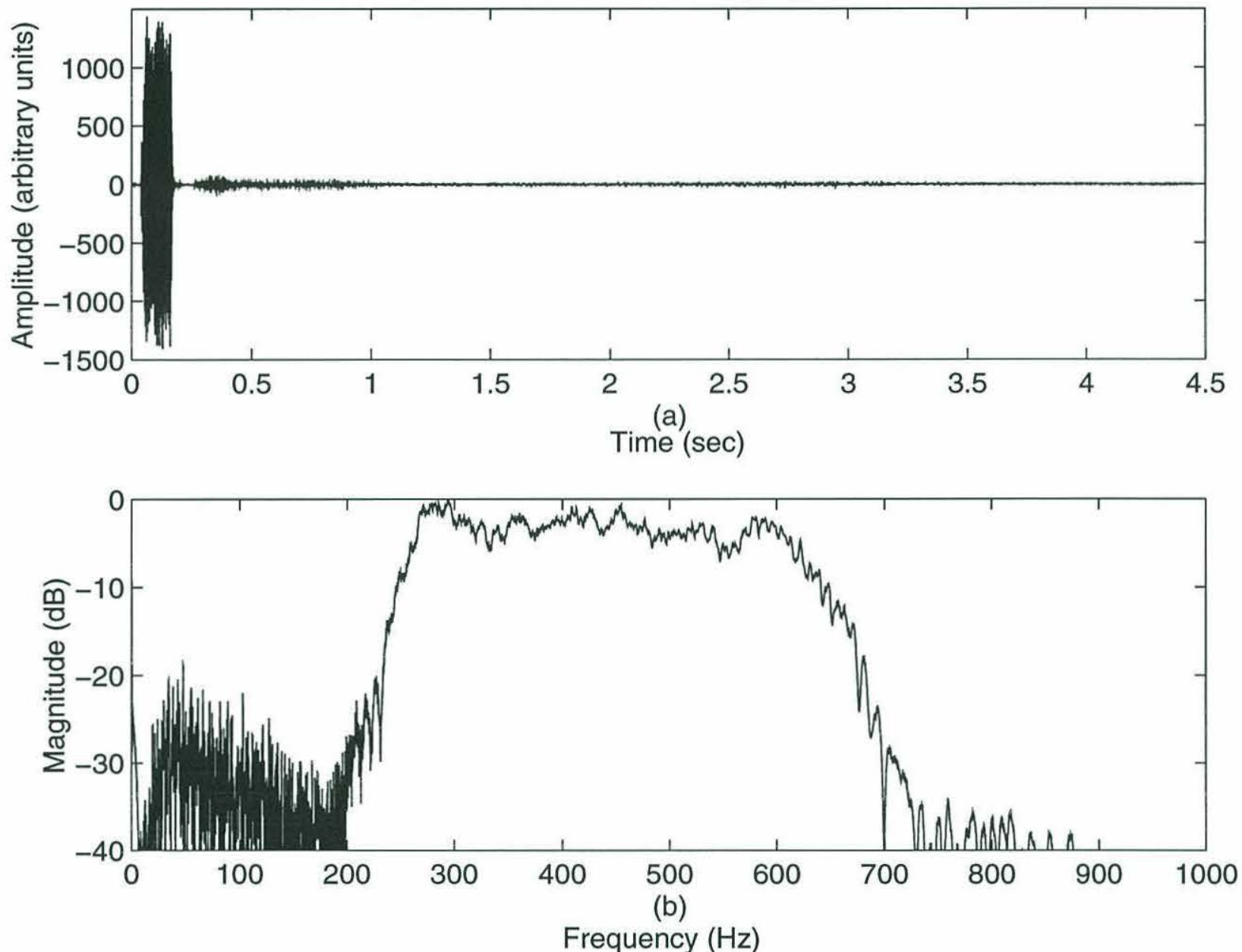

Figure 6-4: (a) The received signal and (b) its spectrum at one hydrophone for a typical ping 


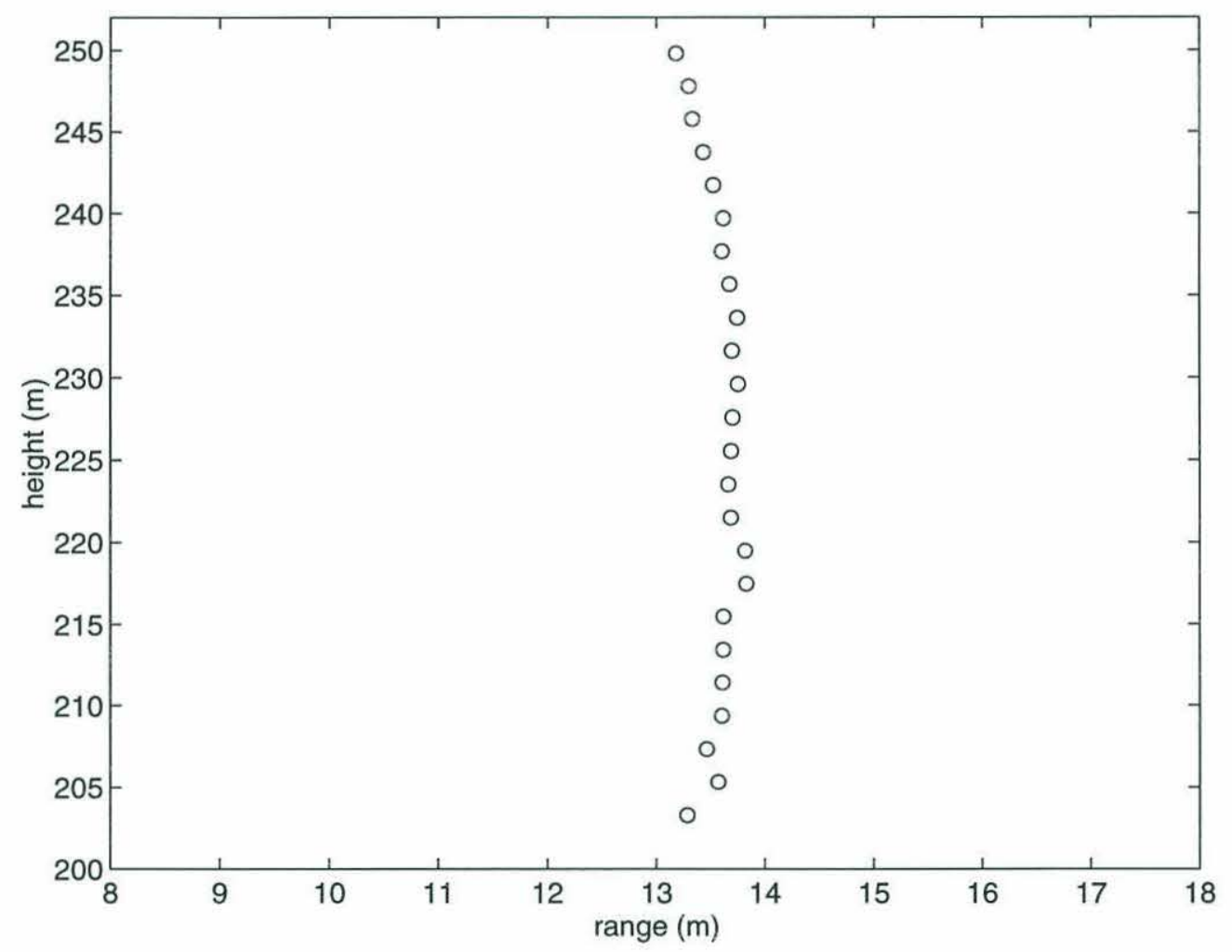

Figure 6-5: The estimated receiving array configuration for a typical ping 


\subsection{Data Processing}

Only a short version of the data processing techniques will be presented here since details have been given in Ref. [10]. The emphasis of this section is on estimating the backscattering strength versus grazing angle from the data.

The sediment is well-known for its layered structure, which is further confirmed by bottom profiling shown in Figs. 1-1 and 1-3 in the experimental region. It is evident from the plot that strong coherent reflections exist at layer interfaces. With the sediment thickness ranging from $100 \mathrm{~m}$ to $400 \mathrm{~m}$, the reflections from deep layers can arrive at the same time as backscattered returns in oblique directions. As a result, the usual time-gating technique are no longer valid in separating the reflected and backscattered returns. In other words, the assumption that scattering arrivals at a certain time are associated with only one scattering angle are not valid. This can explain why the angular dependence of the backscattering strength obtained from a single source/single receiver configuration is error-prone.

The aforementioned problem had been recognized in the design of the ARSRP backscattering experiment and a vertical line array instead of a single hydrophone was used as the receiver. With the beamforming technique, scattered returns in the look direction can be obtained without distortion, while all the returns in other directions have been attenuated. The beam pattern for a conventional single-constraint beamformer is shown in Fig. 6-6. The array here has the same geometry as the actual receiving array depicted in Fig. 6-3, i.e., a 24 element vertical line array with $2 m$ spacing between hydrophones. The frequency is chosen to be the center frequency of the source signal, $450 \mathrm{~Hz}$ and the look direction is -45 degrees. The array response for each circle is $20 \mathrm{~dB}$ higher than that of the neighboring inner circle. The grating lobes in the upper part of the plot are caused by undersampling. From the plotted beam pattern, as expected, one can see that no attenuation is imposed on signals coming from the look direction. However, in sediment volume scattering, the coherent 


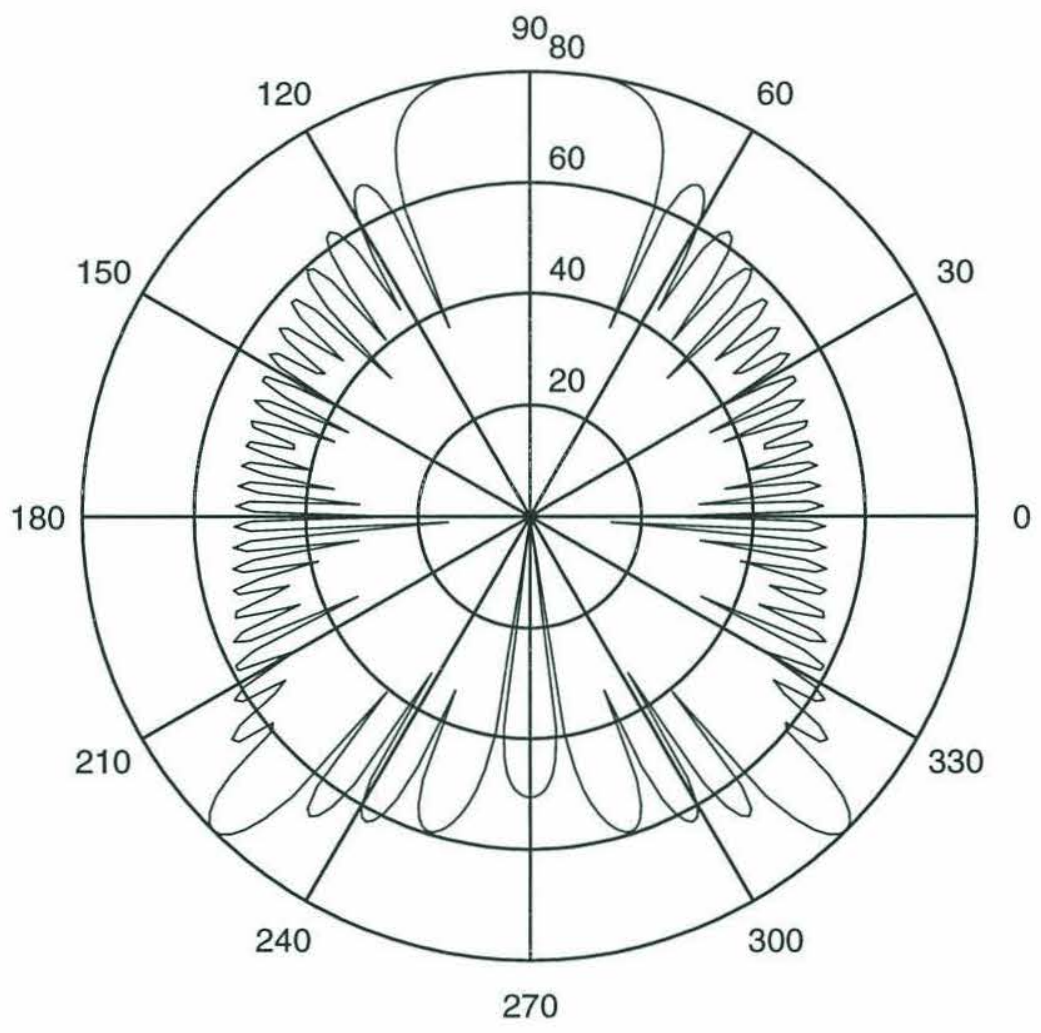

Figure 6-6: The beam pattern for a single-constraint beamformer: frequency $=450$ $\mathrm{Hz}$, look direction $=-45$ degrees. 


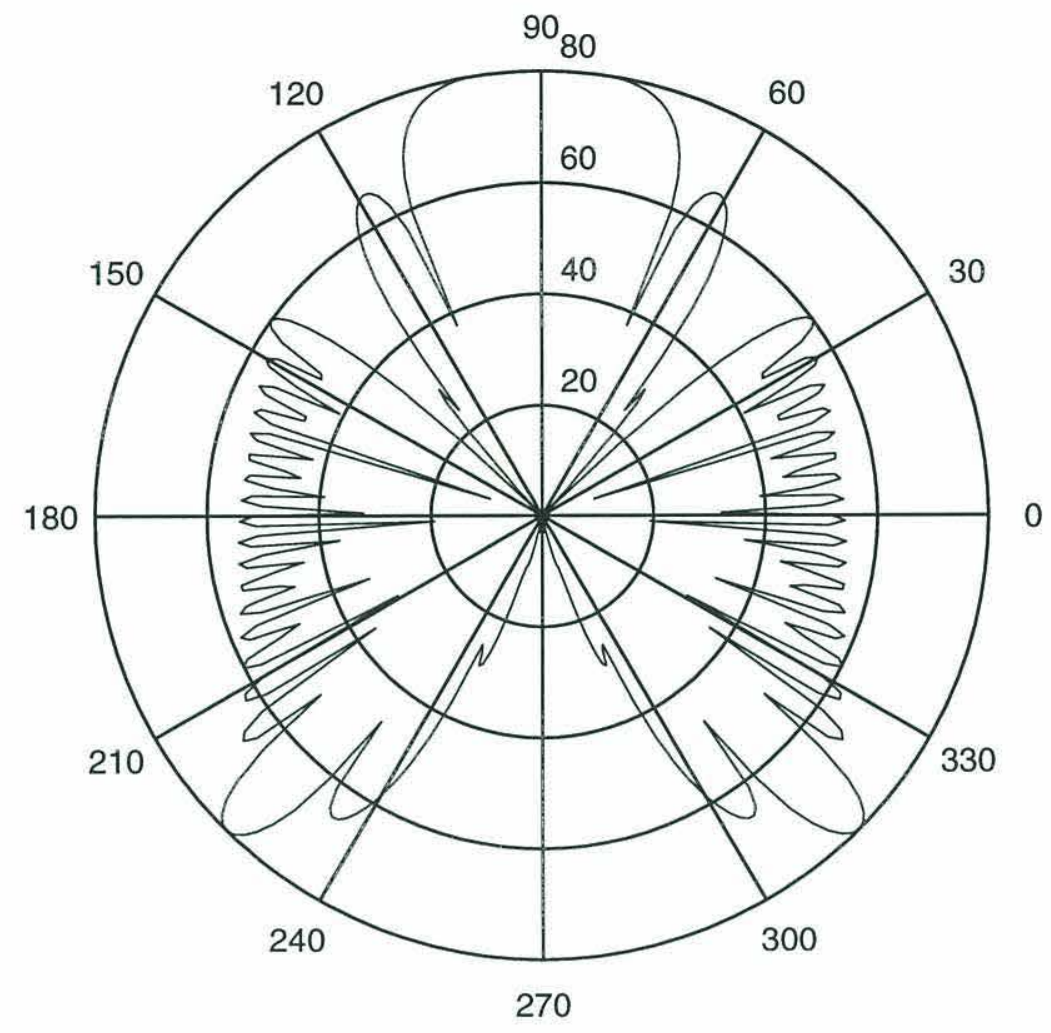

Figure 6-7: The beam pattern for a five-constraints beamformer: frequency $=450$ $\mathrm{Hz}$, look direction $=-45$ degrees

reflections in the normal and near normal incidence directions are much stronger than the backscattered returns in oblique directions, which had been observed in ARSRP backscattering experiment. Therefore, there was a great possibility that the above shown sidelobe levels in the normal and near normal incidence directions were not low enough to reject those unwanted returns. The ineffectiveness of the conventional beamformer had been confirmed by simulations [10]. To overcome this, a multipleconstraints beamformer was developed [10] and its beampattern for the same setup as in the above conventional single-constraint beamformer is shown in Fig. 6-7. A null is formed in the normal and near normal incidence directions, thereby improve the beamformer's ability of alleviating the strong reflections. Simulations in Ref. [10] saw much more favorable results compared to those with conventional single-constraint beamforming. 
To further reduce the normal-incidence returns, a hybrid method, which combines multiple constraints beamforming and a subtraction procedure, was applied in the ARSRP data processing. The idea is to subtract first the strong returns from the normal and near normal incidence directions obtained from endfire beamforming, then apply multiple constraints beamforming to estimate the backscattered returns in oblique directions. Bear in mind that it is case-dependent for the subtraction procedure to work and it is almost impossible to subtract all the returns in the normal and near normal incidence directions for experimental data. However, the subtraction proved to be successful for the ARSRP backscattering data, since most of the high amplitude peaks due to reflections disappeared after the subtraction (cf. Fig. 3-11 [10]). The multiple constraints beamformer will help to reject the residue of the subtraction, which is also in the normal and near normal incidence directions, and obtain the uncontaminated backscattered returns in oblique directions. This led to the findings stated in Chapter 1 that two irregular layers beneath the water/sediment interface are the primary contributors to backscattering in oblique directions.

\subsection{Selection of Model Parameters and Estimation of the Backscattering Strength}

Since the scattering layers are beneath the seafloor, the selection of background parameters for the model such as the sound speed profile and attenuation coefficient would directly affect the estimation of the backscattering strength, which prompts us to address these two together in this section.

The extrapolation of the CTD measurement revealed that the water sound speed at the top of the water/sediment interface is $1530 \mathrm{~m} / \mathrm{s}$. According to Fu et. al. [77], the sound speed just beneath the interface is $1510 \mathrm{~m} / \mathrm{s}$, slightly lower than that in the water column. Using a ray-tracing type of estimation of the sediment/basement 


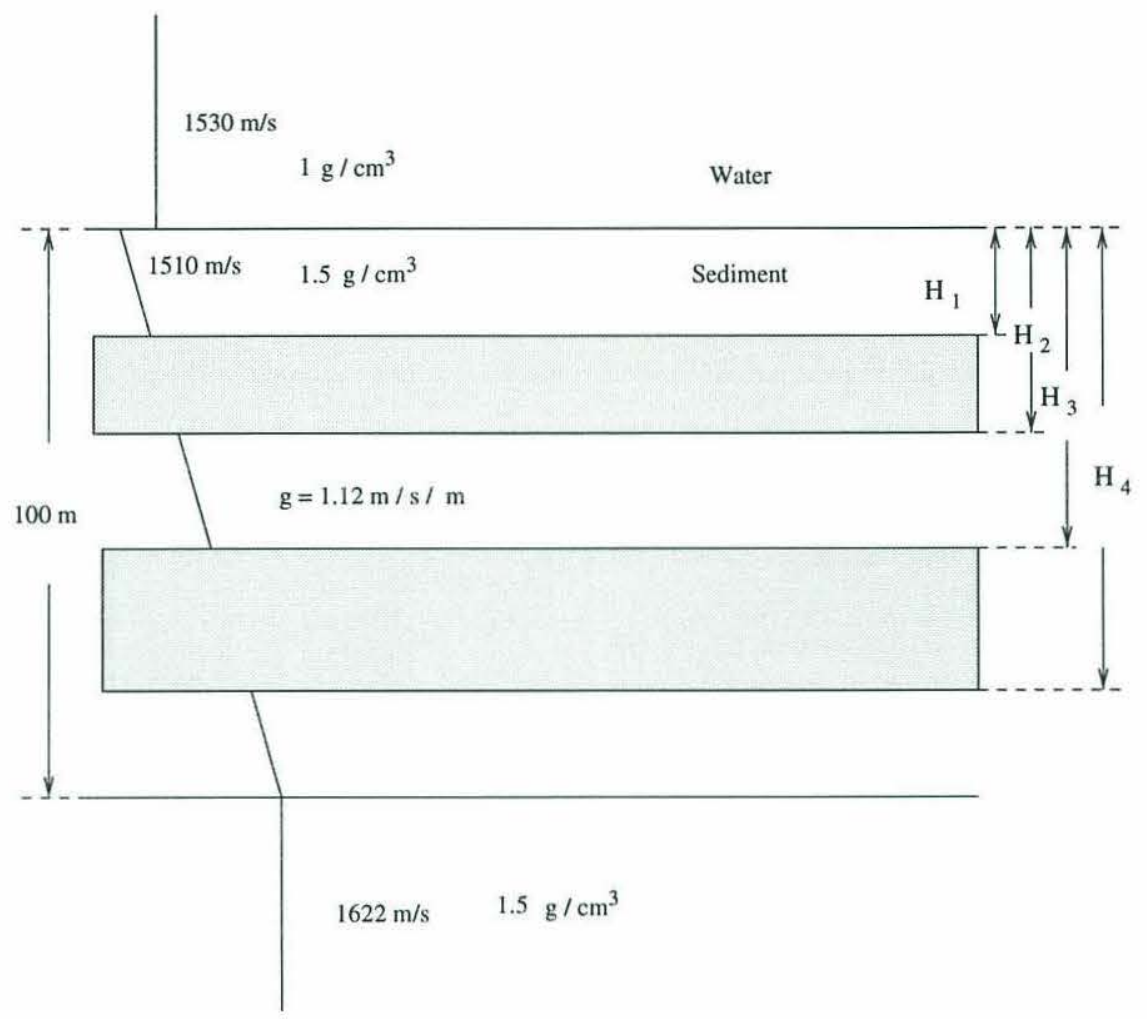

Figure 6-8: Fluid bottom reverberation scenario.

interface returns, the average sound speed gradient in the sediment is found to be approximately 1.12/s [9]. According to Hamilton [62], a typical gradient for this type of sediment is about $1 / s$. From the amplitudes of the normal-incidence reflections at the water/sediment interface and the sediment/basement interface together with the arrival time, the attenuation is approximately estimated as $0.1 \mathrm{~dB} / \lambda$, which is consistent with values in the literature $[59,32]$ for the calcareous mud type of sediment in this area. The density of the sediment is chosen to be $1500 \mathrm{~kg} / \mathrm{m}^{3}$, also well within the range for this type of sediment. By incorporating all the above parameters, we propose a simple fluid bottom model depicted in Fig. 6-8. The sound speed is assumed to be linear from the water/sediment interface down to $100 \mathrm{~m}$. The interface at $100 \mathrm{~m}$ beneath the water/sediment interface is a false one, as is the isovelocity bottom beneath it. As a result, the specular reflections at the sediment/basement interface and their possible insonification of the two irregular scattering layers are neglected because they would arrive later than backscattered returns at 30 degree grazing angle, 
which is the smallest angle at which backscattered returns can still be distinguished from the background noise (cf. Fig. 1-2). The positions of the two irregular layers can be determined from the travel time obtained from bottom profiling shown in Fig. 1-1 together with the above proposed sound speed profile. A simple calculation indicates that the the first irregular layer is located from $H_{1}=15.85 \mathrm{~m}$ to $H_{2}=31.42 \mathrm{~m}$ beneath the water/sediment interface, while the second one is from $H_{3}=56.42 \mathrm{~m}$ to $H_{4}=85.7 \mathrm{~m}$. Notice that these numbers are different from those marked on the side of the bottom profiling plot because a nominal sound speed of $1530 \mathrm{~m} / \mathrm{s}$ was used then. The water/sediment interface was found to be flat as mentioned in the previous section and verified by the data analysis in $[10,13]$, which vindicates the choice of a range-independent water/sediment interface with no roughness in the model. All the above parameters will be the input to OASES so that the Green's function can be evaluated.

To characterize the backscattering process quantitatively, we still resort to estimations of the backscattering strength. As you can recall in Chapter 2, we had discussed the need to be cautious in using the backscattering strength to describe a volume scattering process. This quantity might be inappropriate when multi-path effects are significant. However, the following reasons make us believe that the backscattering strength versus grazing angle is an acceptable parameter in describing backscattering from the two irregular layers found in the ARSRP experiment: First, thanks to the vertical line array and beamforming, we can discriminate the oblique backscattered returns from reflections at layer interfaces, which makes the angular dependence of the estimated backscattering strength trustworthy; second, the multi-path effect is not significant because of a lower sound speed at the top of the sediment compared to that in the water right above it and because of the small sound speed gradient in the bottom; in addition, we are only interested in the angular regime between 30 to 60 degrees grazing angles, where the sediment/basement reflections arrive late as discussed before. 
The estimation of the backscattering strength from data is no simple task. Although the procedures were described in Ref. [10] and reiterated in Chapter 5, we would like to give more details, particularly on how to arrive at the frequency dependence of the backscattering strength. Let's start from Eq.5.1

$$
B S S=10 \log _{10} \frac{I_{s} R^{2}}{I_{\text {in }} A},
$$

where $I_{s}$ is the intensity of the backscattered signals at the reference receiver, $I_{i n}$ is the intensity of the incident sound wave a unit distance away from the scattering volume, $R$ is the distance from the reference receiver to the top of the scattering volume, and $A$ is the top area of the scattering volume. A reference receiver is needed because we now have a vertical line array instead of a single receiver. In this data analysis, the middle hydrophone of the array (the twelfth from the top) is chosen as the reference receiver. Also, the directivity aspect of the array and subsequent beamforming makes it difficult to determine the insonified area precisely. Here, we just consider the simple case where the returns are from the maximum response axis(MRA) of the array, i.e., the returned signals suffer little attenuation passing through the beamformer. As a result, $A$ is estimated by the $3 d B$ area corresponding to the annulus where the array response is within $3 d B$ compared to the MRA. By applying ray tracing, $A$ can be calculated by

$$
A=\pi\left(r_{2}^{2}-r_{1}^{2}\right)
$$

where $r_{1}$ and $r_{2}$ are the horizontal ranges between the reference receiver and the radial bounds of the $3 d B$ area(annulus) at the top of the scattering layer, as shown in Fig. 6-9. Bear in mind that the $3 d B$ beamwidth $\theta_{3 d B}$ and therefore the $3 d B$ insonified area will change with frequency. The lower the frequency, the wider the beam, the larger the insonified area. In order to get the backscattering strength for each frequency, we need to estimate $I_{\text {in }}(f)$ and $I_{s}(f)$, where $f$ stands for the frequency. To calculate $I_{i n}(f)$, the source signal is first Fourier transformed. The amplitude squared of the source spectrum is then multiplied by $R^{2}$ to compensate 


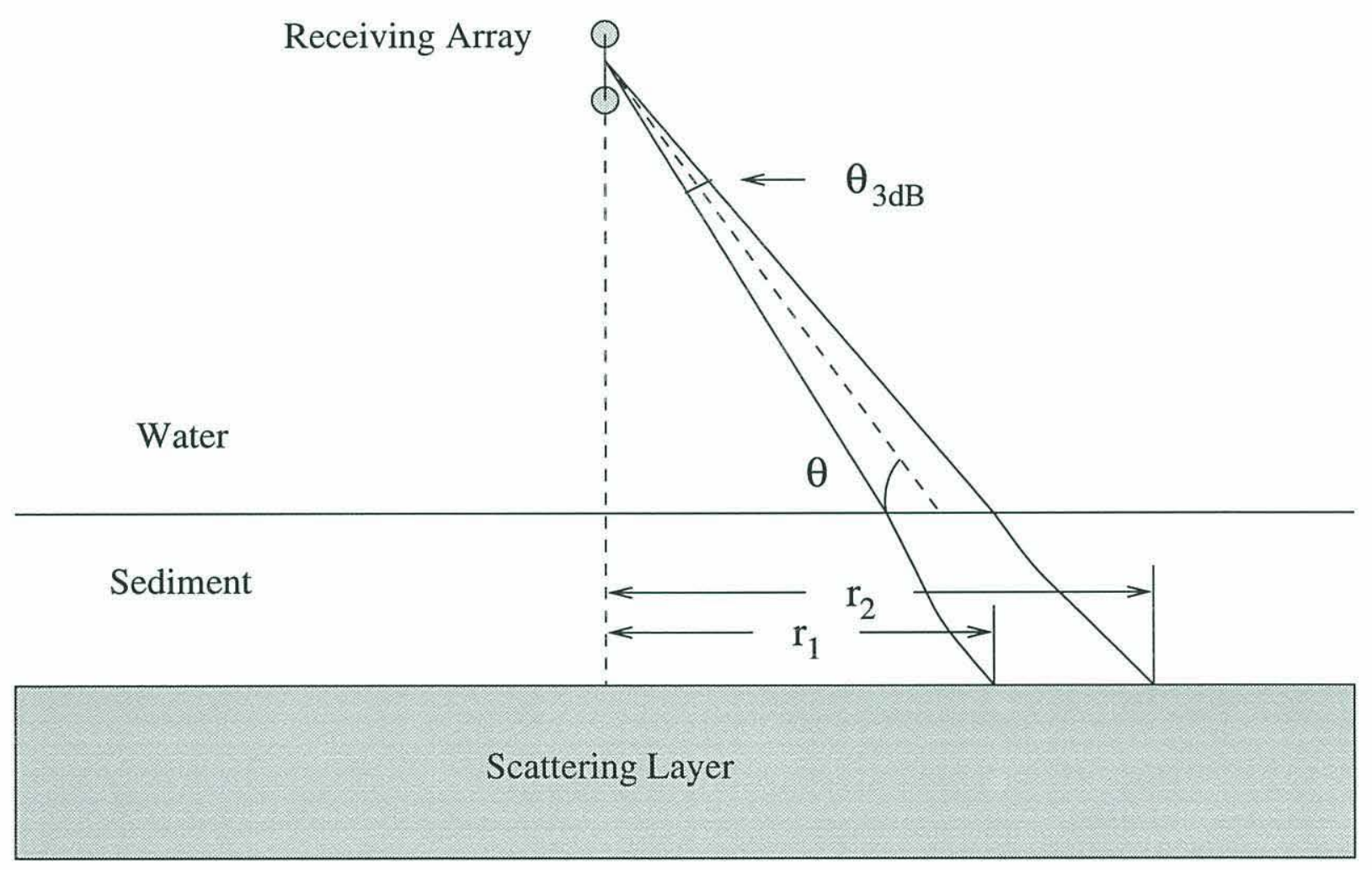

Figure 6-9: Schematic illustration of the scattering strength estimation. 
for the spreading loss. Meanwhile, the calculation of $I_{s}(f)$ would involve one more procedure, which is to determine the exact piece of beamformed data at each angle that corresponds to the backscattered returns from each scattering layer. It can be expressed as follows,

$$
I_{s}(f)=<|F F T\{y(t)\}|^{2}>, \quad T_{1} \leq t \leq T_{2}
$$

where $y(t)$ are the scattered returns, $F F T\{\cdot\}$ stands for the Fourier transform, $|\cdot|$ stands for modulus, $\langle\cdot\rangle$ stands for ensemble average over different pings, and $T_{1}$ and $T_{2}$ are the travel times corresponding to arrivals from the top and the bottom of a scattering layer. Note that $y(t)$ along with $T_{1}$ and $T_{2}$ are different for each scattering angle. The backscattering strength versus grazing angle for each frequency can then be evaluated according to Eq.5.1. The two irregular scattering layers are treated separately. When computing the incident field on the lower irregular layer, the effect of the upper irregular layer on the transmitted field beneath it is neglected. Actually it is consistent with the Born approximation employed in our scattering model since scattering from volume inhomogeneities is supposed to be weak. In Tang's thesis [35], simulations had demonstrated that the transmitted field beneath a six-wavelength thick random layer calculated by the Born approximation was accurate enough. Coincidentally, the upper irregular layer in the ARSRP experiment has a thickness of about six wavelengths. Therefore, the neglect of the influence on the incident field of the lower irregular layer due to the existence of the upper irregular layer can be justified. Taking into consideration the attenuation in the sediment, an amount of $2 \alpha R_{1}$ will be added to the above backscattering strength, where $\alpha$ is the attenuation coefficient and $R_{1}$ is the ray-path length in the sediment.

Figures 6-10 and 6-11 show the estimated backscattering strength versus grazing angle and frequency for the two irregular scattering layers at the east side of the sediment pond. Note that the grazing angle here and from now is the angle measured at the water/sediment interface instead of at the top of the irregular region. How- 


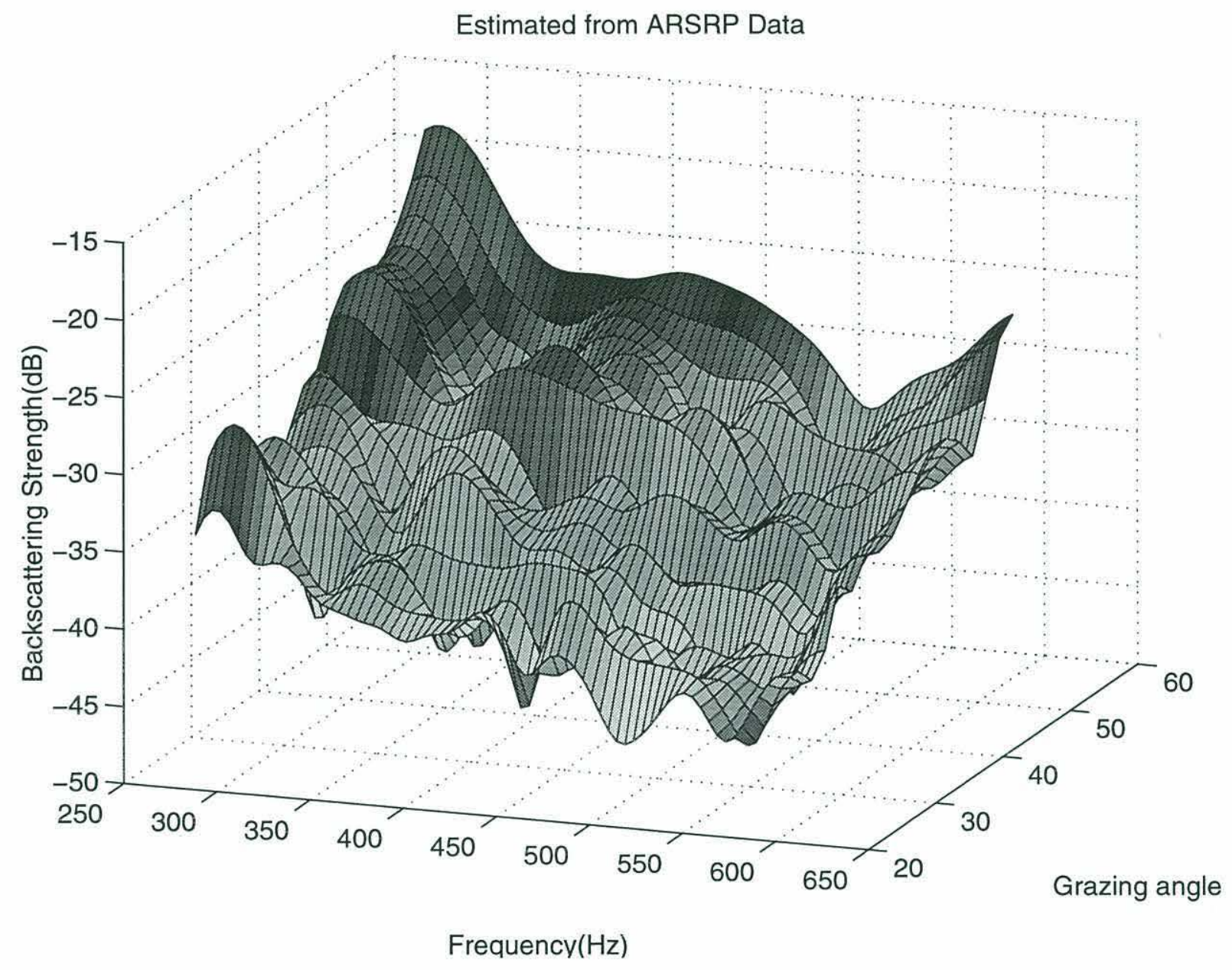

Figure 6-10: The estimated backscattering strength for the upper irregular region at the east side of the sediment pond. 


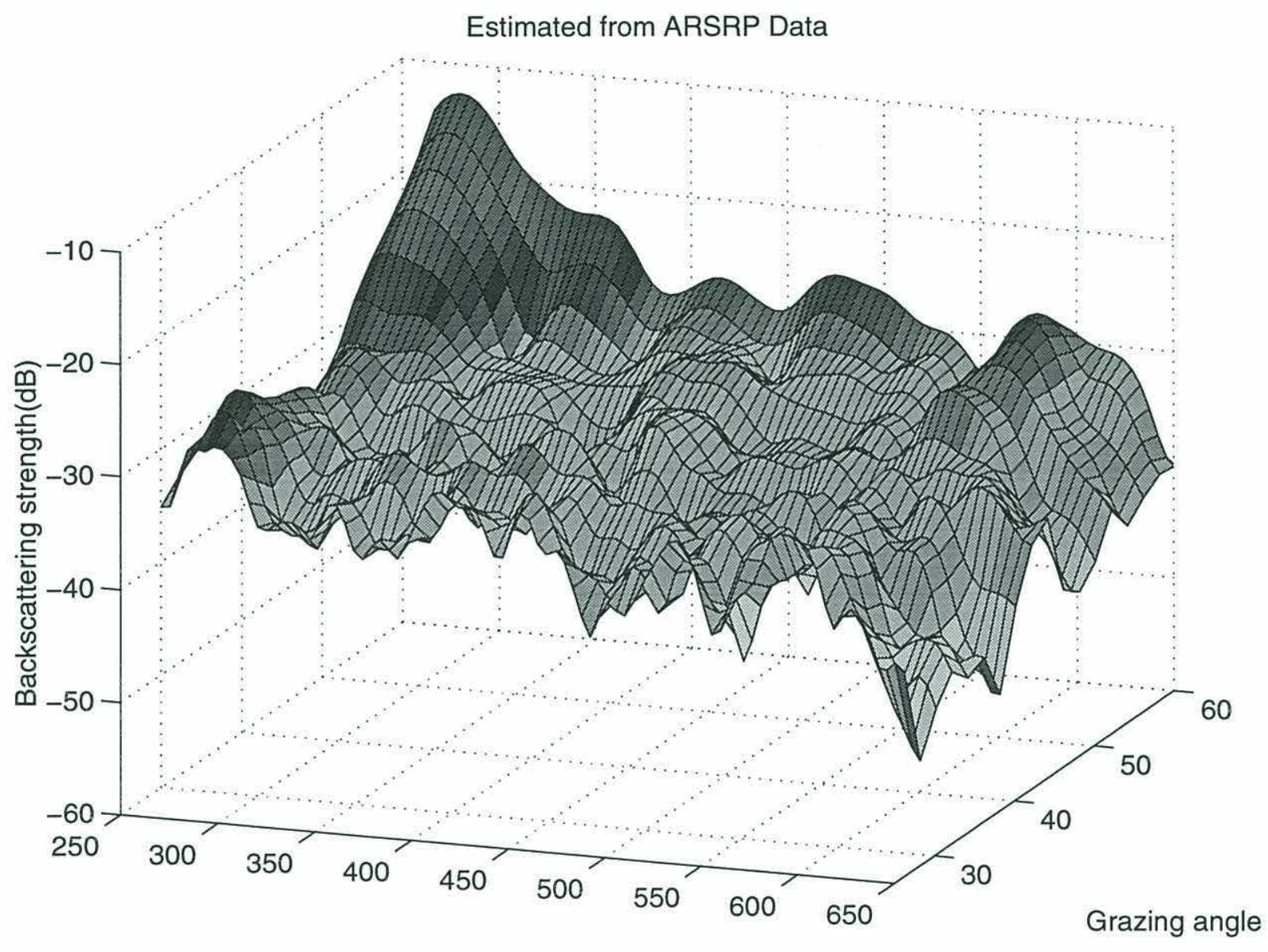

Frequency $(\mathrm{Hz})$

Figure 6-11: The estimated backscattering strength for the lower irregular region at the east side of the sediment pond. 
ever, due to the fact that the sound speed gradient is small, the largest difference, appearing at 30 degrees, is 1.4 degrees for the upper layer and 3.4 degrees for the lower layer, values which are smaller than the beamwidth. Therefore, the angular dependence obtained can be considered as reliable. It can be seen that in general the backscattering strength would decay with the increase of the frequency and/or the decrease of the scattering grazing angle. The widely used Lambert's law will certainly fail to produce the frequency dependence of the backscattering strength. With a point scatter model, the predicted backscattering strength would be independent of scattering angle, which is also not the case here. In Fig. 6-11, there is a peak standing out at low frequencies and high grazing angles. The peak is only confined to grazing angles larger than 50 degrees and frequencies less than $350 \mathrm{~Hz}$, which makes it look more like an artifact. One possible cause of it could be as follows: Although the aforementioned subtraction procedure had eliminated some strong normal and near normal incidence reflections, it can hardly be perfect in a real environment. As a result, there will be some residue or even some error brought in. Certainly the tilting of the layers beneath the lower irregular layer, as can be seen in Fig. 1-1, makes matters worse. The subtraction process would face greater difficulties here. Meanwhile, the beamwidth of the mainlobe is larger for a lower frequency and when the look direction is closer to normal incidence, which increases the chance of picking up those residues in the normal and near normal incidence directions. Incidentally, the returns from the above tilting layers would have about the same arrival time as the scattered returns from the lower irregular layers at about a 60 degree grazing angle. In addition, ambient noise is found to peak between $250 \mathrm{~Hz}$ and $300 \mathrm{~Hz}$, which may influence the scattering strength estimation because of weak oblique backscattering. Therefore, in the model/data comparisons, we will concentrate more on the backscattering strength at frequencies larger than $300 \mathrm{~Hz}$.

Notice that the estimation of the backscattering strength from data is related to the background bottom model, especially the attenuation coefficient. Because the scattered returns from the two irregular layers are identified by their travel times, 


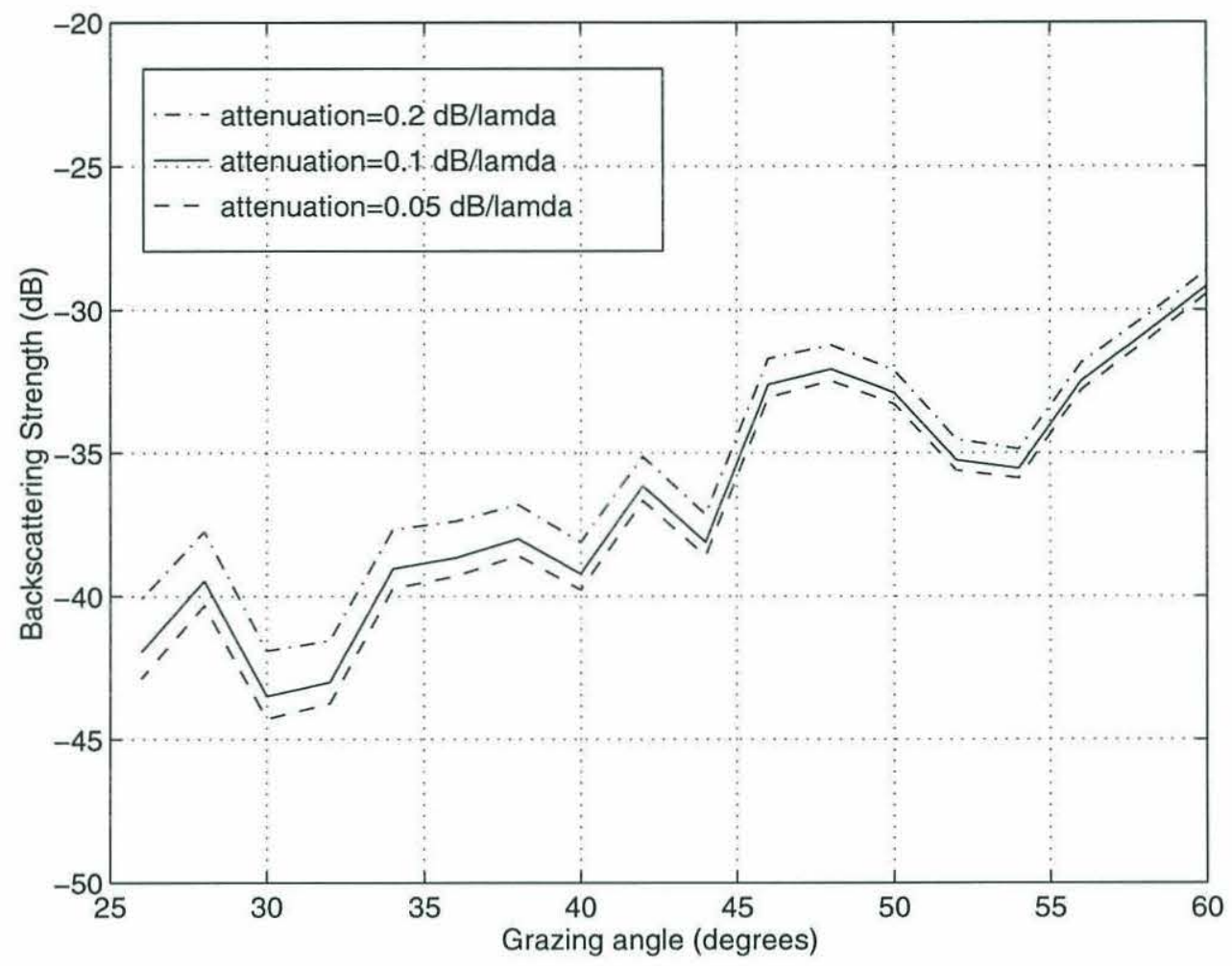

Figure 6-12: The sensitivity of the estimated backscattering strength to the attenuation coefficient at $450 \mathrm{~Hz}$ for the upper irregular layer at the east side of the sediment pond.

errors in the sound speed profile would have little impact on the backscattering estimation. However, the attenuation coefficient would decide the compensation level (cf. p155) of the backscattering strength at different scattering angles, therefore influencing the angular dependence of the backscattering strength as well as the level. In this study, we obtain the best estimate of the attenuation coefficient from the normal incidence returns as $0.1 d B / \lambda$. According to the archival data compiled by Kibblewhite [59], the attenuation coefficient for silt and clay sediment is between $0.01 d B / \lambda$ to $0.12 d B / \lambda$. The example shown in Fig. 6-12 illustrates the sensitivity of the estimated backscattering strength at $450 \mathrm{~Hz}$ to the attenuation coefficient. 


\subsection{Model and Data Comparison}

By no means it is an easy task computationally to generate scattering time series, especially the wide $400 \mathrm{~Hz}$ bandwidth in the ARSRP experiment. Adding to the degree of difficulty are the thick scattering layer and fine sampling requirement. In order to perform beamforming, scattered returns have to be simulated across the whole receiving array. Therefore, instead of a scattering time series, we choose to compare the backscattering strength versus grazing angle estimated from model simulation and real data for a selected set of frequencies over the signal spectrum.

The fact that multiple constraints beamforming is carried out in the frequency domain provides us with extra benefit. In other words, if the scattered field at each hydrophone for a single frequency is available, we are still able to perform beamforming to obtain the scattered field and therefore the scattered intensity at different scattering angles. As a result, in order to compare with data, all we need to do in the modeling part is to simulate the scattered field at each receiver for the selected set of frequencies. The beamforming is the same as that in the data processing. So is the estimation of the backscattering strength after obtaining the backscattering intensity for each frequency. Meanwhile, the procedures of simulating the scattering time series have been given in Chapter 5. We simply forego the last step, the Fourier synthesis, since our goal here is to obtain the scattered field at certain frequencies.

Model/data comparisons will be carried out for the two irregular layers at the east side of the sediment pond only. We choose to compare at six frequencies: $300 \mathrm{~Hz}$, $350 \mathrm{~Hz}, 400 \mathrm{~Hz}, 450 \mathrm{~Hz}, 500 \mathrm{~Hz}$, and $550 \mathrm{~Hz}$. As for the power spectrum and characteristic length scale to describe the inhomogeneous sound speed and density fluctuations, we elect to test the Gaussian distribution and the power law distribution (the exponential distribution is a special case of a power law distribution). There is no evidence suggesting horizontal anisotropy in the ARSRP Site A sediment pond area. So we will simply let the horizontal correlation length $l_{x}=l_{y}$ in the model. 
Meanwhile, there is no measurement of the correlation length in this sediment area. By examining Fig. 1-1, one would have the impression that the random field is much better correlated horizontally than vertically. As a matter of fact, this is consistent with the geoacoustical model proposed by Lysanov [29], in which he suggested largescale in the horizontal plane and small-scale in depth for random inhomogeneities in the sediment, the so-called "pancake" model. The above sediment profiling covered a horizontal distance of about $330 \mathrm{~m}$. The rough estimation would show us that the horizontal correlation length be on the order of $10 \mathrm{~m}$. Of course, the estimation can be very wrong. However, it is just the starting point of our parameter search. In the meantime, the standard deviation $\sigma$ of the sound speed fluctuation can range from $1.5 \%$ to $8 \%$ and $\beta$, the ratio between density and sound speed fluctuations, between 1 to 10 according to Yamamoto [64]. Also in Yamamoto's estimation, both sound speed and density fluctuations were characterized by a power law distribution with $\nu$ (cf. Eq.3.35) between 0.4 and 0.65 .

As shown in Fig. 6-13, the best fit for the upper irregular layer is with a power law distribution. The parameters for the model are correlation lengths $l_{x}=l_{y}=20 \mathrm{~m}$, $l_{z}=0.7 m$, the standard deviation of sound speed fluctuations $\sigma=2.8 \%, \beta=3$, and $\nu=0.5$. The model predicted curves are ensemble averages of 200 realizations, therefore the standard deviations are very small. (see Appendix B for the estimation of the standard deviation from the simulation and data.) In general, the model curves agree with the data very well. Notice that in the data curves, around a 47 degree grazing angle for all the selected frequencies except $500 \mathrm{~Hz}$, there is a small bump. No specific cause has been found for this and the model cannot predict it either. As for the parallel shift between the model and data curves at $300 \mathrm{~Hz}$, it might be due to the aforementioned strong ambient noise. Figure 6-14 shows the model/data comparison for the lower irregular layer at the east side of the sediment pond. The best fit again belongs to random sound speed fluctuations with a power law type of power spectrum. The horizontal correlation lengths are $l_{x}=l_{y}=20 \mathrm{~m}$, the vertical correlation length is $0.8 m$, the standard deviation $\sigma=2 \%, \beta=3$, and 

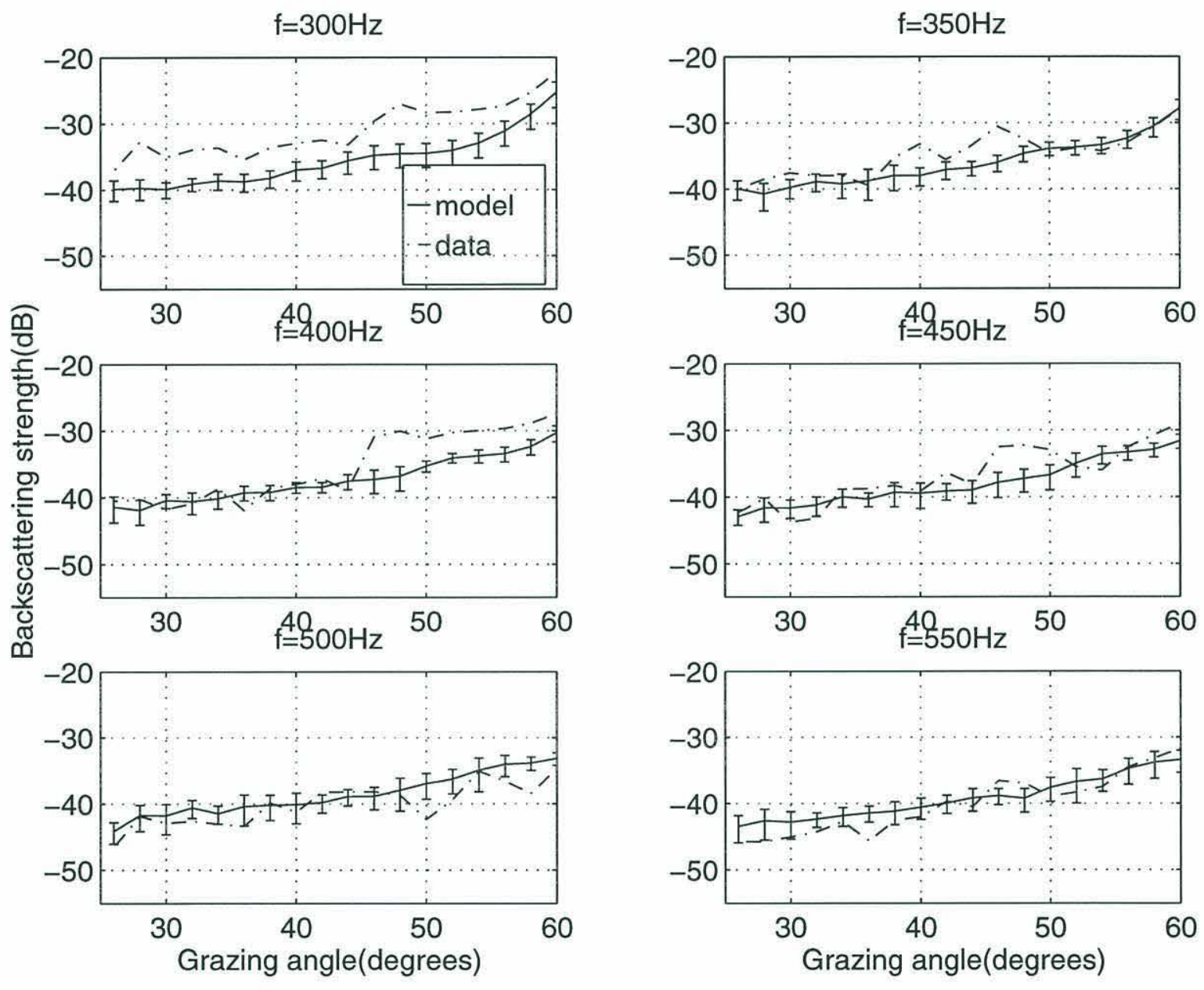

Figure 6-13: Model/data comparison for the upper irregular layer at the east side of the sediment pond. Sound speed fluctuations are described by a power law distribution with $l_{x}=l_{y}=20 m, l_{z}=0.7 m, \sigma=2.8 \%, \beta=3$, and $\nu=0.5$. The error bars show the standard deviation of the simulated backscattering strength. 

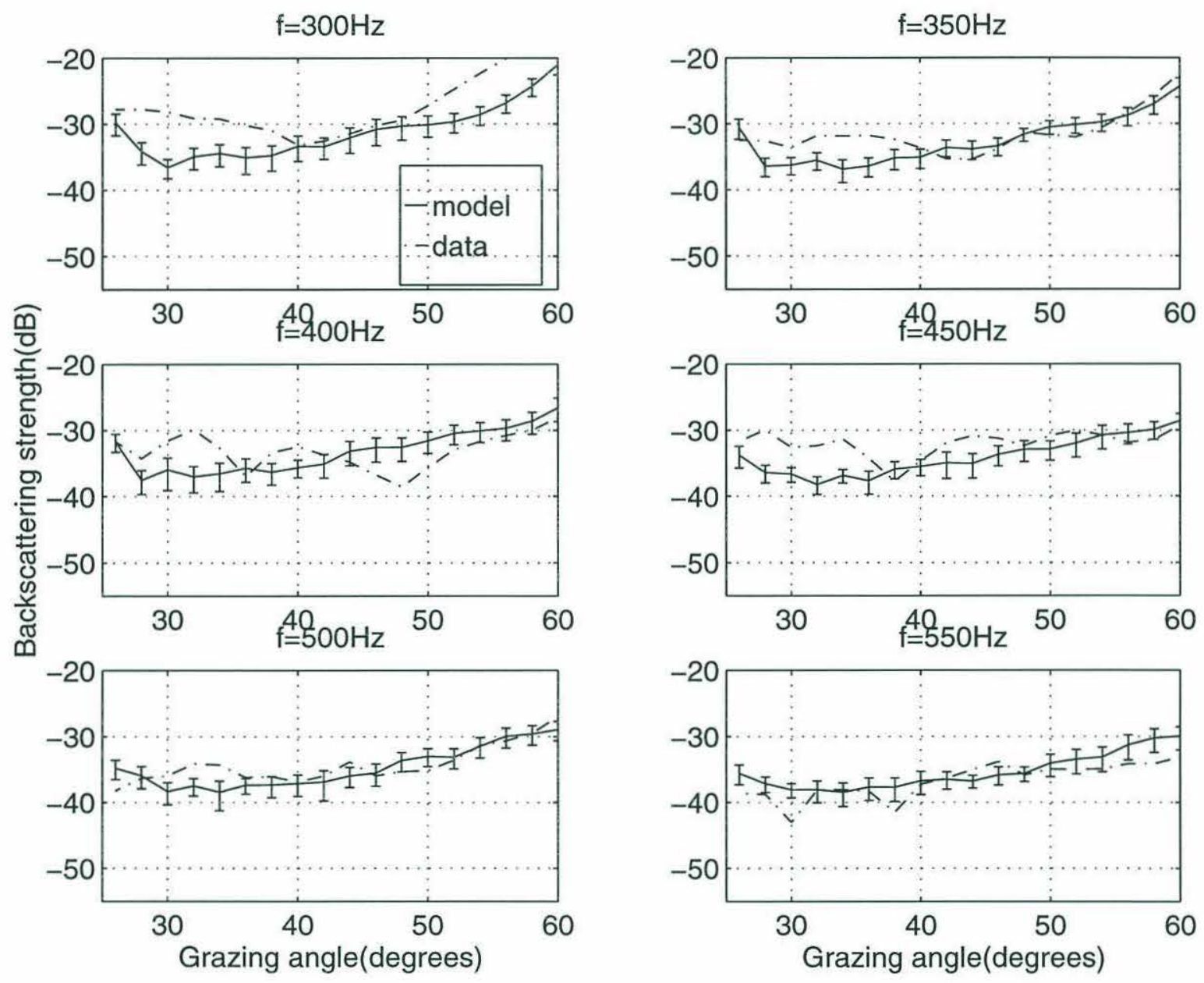

Figure 6-14: Model/data comparison for the lower irregular layer at the east side of the sediment pond. Sound speed fluctuations are described by a power law distribution with $l_{x}=l_{y}=20 \mathrm{~m}, l_{z}=0.8 \mathrm{~m}, \sigma=2 \%, \beta=3$, and $\nu=0.5$. The error bars show the standard deviation of the simulated backscattering strength. 
$\nu=0.5 .200$ realizations are used to obtain the ensemble average. The matches are again satisfactory, although the error in sound speed profile and attenuation estimation would have more impact here than on the comparison of the upper irregular layer.

The effects of error in the background bottom model upon the backscattering strength predicted by the model is minimal because the propagation effects associated with the background bottom model is cancelled out in estimating the backscattering strength.

For the Gaussian distribution, an acceptable fit for the upper irregular layer is obtained with $l_{x}=l_{y}=1 m, l_{z}=0.2 m, \sigma=1 \%$, and $\beta=2$. To us, these parameters are less reasonable. The agreements are not as good as with a power law distribution, either. In addition, the fit is far less satisfactory for the lower layer. All of these factors make the idea of using a Gaussian distribution to describe sound speed and density fluctuations unfavorable to us in the ARSRP backscattering scenario.

As discussed in Chapter 3, for backscattering from random inhomogeneities, the angular dependence of the backscattering strength is mainly determined by the rolloff rate of its power spectrum versus wavenumber. This is because the roll-off rate is directly related to the level of small-scale roughness at high wavenumbers. For a power law distribution, the quantity $\nu$ will control the roll-off rate $(2(\nu+3 / 2)$ for a 3-D case) and therefore the angular dependence. Different correlation lengths will only alter the power spectrum level at a particular wavenumber but not the roll-off rate. The aspect ratio, defined by the horizontal correlation length over the vertical correlation length, would play a role as well, although not as significant as $\nu$, which is shown in Fig. 6-15. Figure 6-16 shows the results of the parameter study. The two curves have the same $\nu, \sigma, \beta$, same aspect ratio but different correlation lengths. It can be seen that the trends of the two curves are very similar but not exactly the same. The differences can be seen at $450 \mathrm{~Hz}$ and $550 \mathrm{~Hz}$, for example. Although we find the dash line the best fit with data, it is a close call since the differences are small 

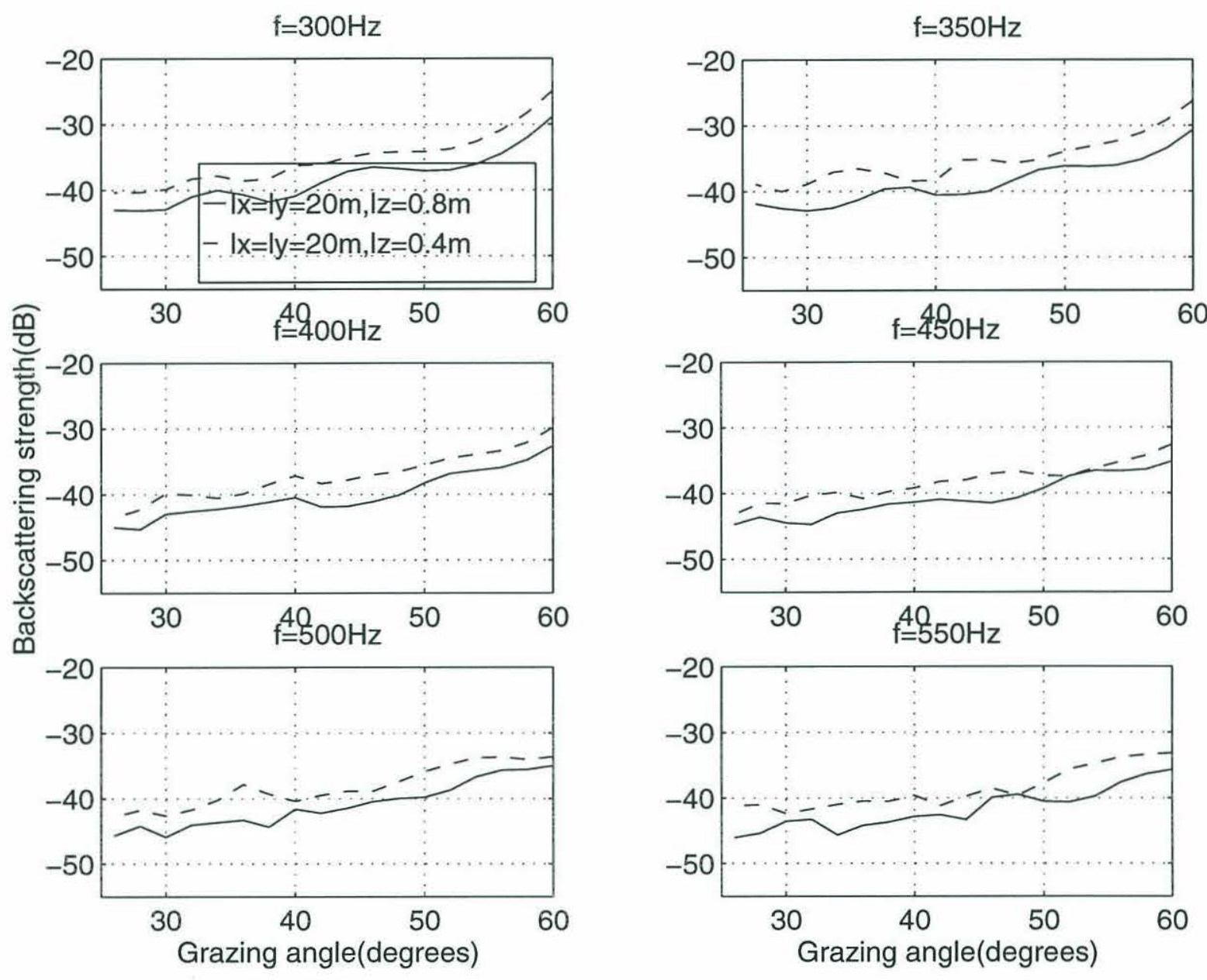

Figure 6-15: Parameter studies for sound speed and density fluctuations described by a power law type of power spectrum: the effect of the aspect ratio. 

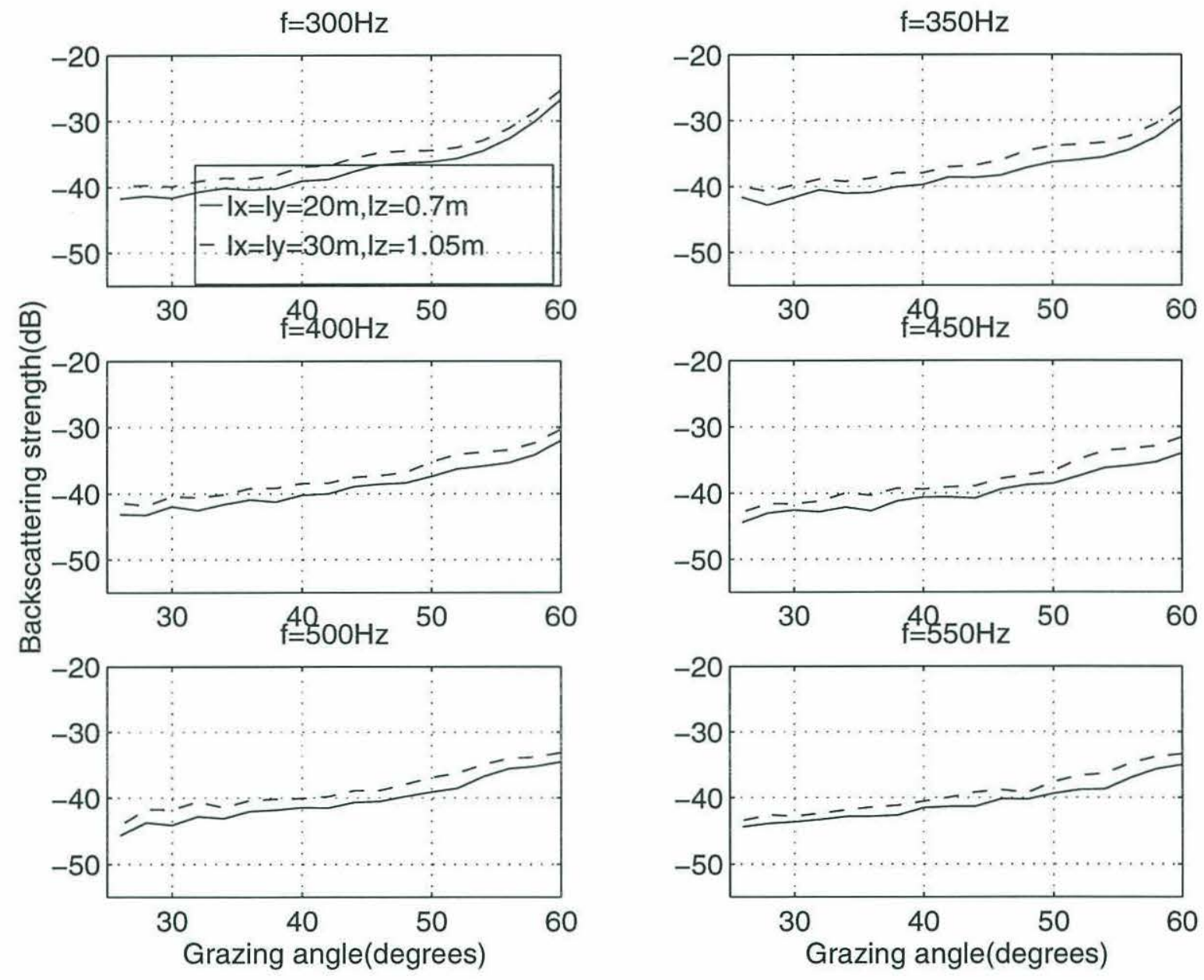

Figure 6-16: Parameter studies for sound speed and density fluctuations described by a power law type of power spectrum: same aspect ratio but different correlation lengths, $\nu=0.5$. 
. This insensitivity would make it difficult to invert for sediment properties such as correlation lengths from the angular dependence of the backscattering strength. On the other hand, it reveals the importance of estimating $\nu$ if random inhomogeneities are described by a power law distribution. In the meantime, it would benefit the task of predicting the directivity of the backscattered field without full knowledge of the ground truth.

In summary, the model is able to match the ARSRP backscattering data well with a power law type of power spectrum description of random sound speed and density fluctuations. Yet, model/data comparisons for more data sets are needed before any conclusion reached in this section can be generalized. Nevertheless, the successful model/data comparison does show the merit of our volume backscattering model. 


\section{Chapter 7}

\section{Conclusions and Future Work}

In this thesis an attempt has been made to model monostatic backscattering from 3-D volume inhomogeneities in the sediment and to compare the results of the model with ARSRP backscattering data. While the work presented here is helpful in understanding the underlying physics and predicting the scattering phenomena, more work remains to be carried out in order to achieve one of our ultimate goals, solving the inverse problem. In this chapter, we will try to draw some general conclusions from the work being done and come up with some suggestions for future research.

\subsection{Conclusions}

The results of the thesis can be summarized as follows:

- A scattering process cannot be modeled correctly without properly accounting for the incident field. The scattered field and the incident field are interrelated and actually it is the total field, i.e., the combination of the two, that controls the reradiation of acoustic energy in an inhomogeneous medium. In some scenarios, the scattered field is much weaker than the incident field, and therefore the total field can be approximated by the incident field (i.e., the Born approximation). In any case, an accurate evaluation of the incident field is essential in modeling the scattering process. As revealed in Chapter 2, several approximate propagation models used in volume scattering studies sometimes 
have their drawbacks in dealing with scattering from a bottom with complicated sound speed structure where multi-path effects are significant. As a matter of fact, not only do we need to examine the accuracy of these approximation methods in different scenarios, but also it is often necessary to evaluate the incident field exactly by either an analytical solution or a numerical method such as wavenumber integration. The above results are obtained through simulations in a deep-water environment. In shallow water, we would expect the propagation of sound waves become more intricate because of the waveguide effect [7], which would further the requirement of a good propagation model so as to calculate the incident field accurately in scattering modeling.

- With OASES chosen as the propagation model in this work, we are provided with the ability to model volume scattering from a bottom with complicated sound speed structures such as sound speed gradient, multi-layering, and even an elastic basement as long as the scatterers are located in a fluid layer.

- The concept of applying the equivalent surface backscattering strength in characterizing volume scattering processes is still valid in some high-frequency and high-attenuation bottom scattering studies. However, precautions need to be taken when multi-path contributions to the field are evident, which would put the obtained angular dependence of the equivalent surface backscattering strength in question.

- The volume scattering model described in Chapter 3 provides a tool to model scattering contributions from both sound speed and density fluctuations in a volume. Simulations in Chapter 5 have confirmed the fact that density variations are dominant in backscattering. Meanwhile, the analytic solutions in a free-space scenario show clearly that the nature of the scattered field depends strongly on the statistical distribution of the random sound speed/density variations. Both the characteristic length scale and the power spectrum descriptions of the random inhomogeneities have great impact on the directionality of the scattered field. Although the monostatic configuration cannot resolve 
the anisotropic nature of the scattered field in horizontal planes, the angular dependence would behave very differently because of that. Effects due to the vertical correlation of the random scatterers are evident in the numerical experiments. To model the scattering volume as vertically uncorrelated stacks of layers would bear the risk of losing important contributions due to the vertical correlation. All the above helps us to reach the conclusion that it is critical to obtain ground truth for the sound speed/density variations in the sediment volume. The effort of achieving better understanding and parameterizing the variations of the sediment properties should be an integrated part of volume scattering studies.

- Simulating scattering from full 3-D volumetric inhomogeneities is still very computionally expensive. In Chapter 4 , the monostatic backscattering configuration has been taken advantage of so that the azimuthally-summed 3-D random field can be generated with much relaxed computational requirements. It enables us to get a grip on the full scope of monostatic volume backscattering in a real environment.

- In the model and ARSRP backscattering data comparison, a power law distribution of random sound speed/density variations is found to fit the data very well. Parameter wise, on the one hand, the horizontal correlation length is much larger than the vertical correlation length, which is consistent with some of the geophysical models of the sediment (so-called "pancake" models). On the other hand, the change of horizontal correlation length in a power law distribution does not have strong effect on the slope of the backscattering strength versus grazing angle but only the level. Rather the slope is determined by the decaying rate of the corresponding power spectrum versus wavenumber, i.e., the fractal dimension, which is directly related to the sound speed/density fluctuations in small scales. This would benefit people who want to predict scattering phenomena but only with limited knowledge of the sediment properties. However, this insensitivity presents a challenge for people who attempt to do inversion. All in 
all, this first-order volume scattering model has been proved capable of matching the experimental data and the power law distribution is found to describe the sound speed and density fluctuations in the sediment satisfactorily.

\section{2 $\quad$ Future Work}

There are a number of ways to improve or extend the proposed model in this thesis.

- A logical next step would be to take advantage of the ability rendered by OASES to include rough surface scattering in the picture. The water/sediment interface scattering was insignificant in the ARSRP backscattering experiment. However, a more general scenario would inevitably involve both rough surface scattering and volume scattering. It would be interesting to see the combined effect and to compare that with surface scattering or volume scattering only.

- The volume scattering model that we have here is essentially a single scattering model, which would certainly be inaccurate when multiple scattering is of concern. The development of a multiple scattering model will improve our modeling ability and further our understanding of the scattering mechanisms.

- A very useful extension of this work would be to model bistatic scattering instead of only monostatic backscattering, although we may not be able to enjoy the benefit of a simplified 3-D random field generation.

- The work present above models volume scattering in fluid media only and cannot account for scattering from an elastic bottom. The model would be more useful if it could describe volume scattering in elastic media, which has attracted more attention recently.

- If an experiment similar to the ARSRP experiment were to be performed in the future, more effort in gathering ground-truth information is highly recommended, especially the attenuation coefficient. 


\section{Appendix A}

\section{The Variance and Power Spectral}

\section{Density of the 2-D}

\section{Azimuthally-Summed Random}

\section{Field with Gaussian Correlation}

\section{Function}

As mentioned in Chapter 4, the 2-D Gaussian correlation function in Cartesian coordinates is

$$
C\left(\xi_{x}, \xi_{y}\right)=\sigma^{2} \exp \left(-\frac{1}{2}\left[\frac{\xi_{x}^{2}}{l_{x}^{2}}+\frac{\xi_{y}^{2}}{l_{y}^{2}}\right]\right),
$$

where $\sigma$ is the standard deviation, and $l_{x}$ and $l_{y}$ are the correlation lengths in the $x$ and $y$ directions, respectively. The corresponding power spectral density is

$$
W\left(k_{x}, k_{y}\right)=2 \pi l_{x} l_{y} \exp \left(-\frac{k_{x}^{2} l_{x}^{2}+k_{y}^{2} l_{y}^{2}}{2}\right) .
$$


For an isotropic random field, we have $l_{x}=l_{y}=l$. After the coordinate transformation

$$
\left\{\begin{array}{l}
x=r \cos \phi \\
y=r \sin \phi
\end{array}\right.
$$

the correlation function of $\epsilon$ becomes

$$
<\epsilon\left(r_{1}, \phi_{1}\right) \epsilon^{*}\left(r_{2}, \phi_{2}\right)>=\exp \left(-\frac{r_{1}^{2}+r_{2}^{2}-2 r_{1} r_{2} \cos \left(\phi_{1}-\phi_{2}\right)}{2 l^{2}}\right),
$$

where $(*)$ stands for the conjugate operation. Since

$$
\eta(r)=\int_{0}^{2 \pi} \epsilon(r, \phi) d \phi
$$

the correlation function of $\eta$ becomes

$$
\begin{aligned}
<\eta\left(r_{1}\right) \eta^{*}\left(r_{2}\right)> & =\int_{0}^{2 \pi} \int_{0}^{2 \pi}<\epsilon\left(r_{1}, \phi_{1}\right) \epsilon^{*}\left(r_{2}, \phi_{2}\right)>d \phi_{1} d \phi_{2} \\
& =\sigma^{2} \exp \left(-\frac{r_{1}^{2}+r_{2}^{2}}{2 l^{2}}\right) \int_{0}^{2 \pi} \int_{0}^{2 \pi} \exp \left(\frac{r_{1} r_{2} \cos \left(\phi_{1}-\phi_{2}\right)}{l^{2}}\right) d \phi_{1} d \phi_{2} \\
& =4 \pi^{2} \sigma^{2} I_{0}\left(\frac{r_{1} r_{2}}{l^{2}}\right) \exp \left(-\frac{r_{1}^{2}+r_{2}^{2}}{2 l^{2}}\right) .
\end{aligned}
$$

If $r_{1}=r_{2}=r$, the variance at range $r$ is

$$
<\eta(r) \eta^{*}(r)>=4 \pi^{2} \sigma^{2} I_{0}\left(\frac{r^{2}}{l^{2}}\right) \exp \left(-\frac{r^{2}}{l^{2}}\right) .
$$

For an anisotropic random field, the correlation function of $\eta(r)$ is

$$
\begin{aligned}
& <\eta\left(r_{1}\right) \eta^{*}\left(r_{2}\right)>= \\
& \sigma^{2} \int_{0}^{2 \pi} \int_{0}^{2 \pi} \exp \left(-\left[\frac{\left(r_{2} \cos \phi_{2}-r_{1} \cos \phi_{1}\right)^{2}}{2 l_{x}^{2}}+\frac{\left(r_{2} \sin \phi_{2}-r_{1} \sin \phi_{1}\right)^{2}}{2 l_{y}^{2}}\right]\right) d \phi_{1} d \phi_{2} .
\end{aligned}
$$


The variance as a function of $r$ in this case becomes

$$
\begin{aligned}
& \operatorname{Var}\{\eta(r)\}= \\
& \quad \sigma^{2} \int_{0}^{2 \pi} \int_{0}^{2 \pi} \exp \left(-\frac{r^{2}}{2 l_{x}^{2} l_{y}^{2}}\left[l_{y}^{2}\left(\cos \phi_{2}-\cos \phi_{1}\right)^{2}+l_{x}^{2}\left(\sin \phi_{2}-\sin \phi_{1}\right)^{2}\right]\right) d \phi_{1} d \phi_{2} .
\end{aligned}
$$

The power spectral density $W_{s}$ is as follows

$$
\begin{aligned}
W_{s}\left(k_{r}\right) & =2 \pi l_{x} l_{y} \int_{0}^{2 \pi} \exp \left(-\frac{k_{r}^{2}\left(l_{x}^{2} \cos ^{2} \theta+l_{y}^{2} \sin ^{2} \theta\right)}{2}\right) d \theta \\
& =2 \pi l_{x} l_{y} \exp \left(-\frac{k_{r}^{2}\left(l_{x}^{2}+l_{y}^{2}\right)}{4}\right) \int_{0}^{2 \pi} \exp \left(-\frac{k_{r}^{2}\left(l_{x}^{2}-l_{y}^{2}\right) \cos 2 \theta}{4}\right) d \theta \\
& =4 \pi^{2} l_{x} l_{y} \exp \left(-\frac{k_{r}^{2}\left(l_{x}^{2}+l_{y}^{2}\right)}{4}\right) I_{0}\left(\frac{k_{r}^{2}\left(l_{x}^{2}-l_{y}^{2}\right)}{4}\right) .
\end{aligned}
$$




\section{Appendix B}

\section{Estimation of the Standard}

\section{Deviation}

The standard deviation is widely used to describe the dispersion of a random distribution. It is known to work best for a Gaussian distribution. However, the values of the standard deviation is greatly affected by outliers for some non-Gaussian distributions and therefore may be a poor measure of dispersion in those cases. The formula to estimate the standard deviation from random realizations is

$$
S T D\{y\}=\sqrt{\frac{1}{N-1} \sum_{i=1}^{N}\left(y_{i}-\bar{y}\right)^{2}},
$$

where $N$ represents the number of realizations and $\bar{y}$ the mean of $y$.

For the simulated backscattering coefficients from the model in Chapter 6 (a power law distribution), the distribution is not Gaussian but more like an exponential distribution (or a Chi-square distribution with 2 degrees of freedom), which can be seen in Fig. B-1. It is clear that in this case the standard deviation no longer qualifies as a quantitative measurement of the dispersion.

The backscattering coefficient estimated from the data is the mean of eight pings (eight independent realizations). If the mean value is treated as a new random vari- 


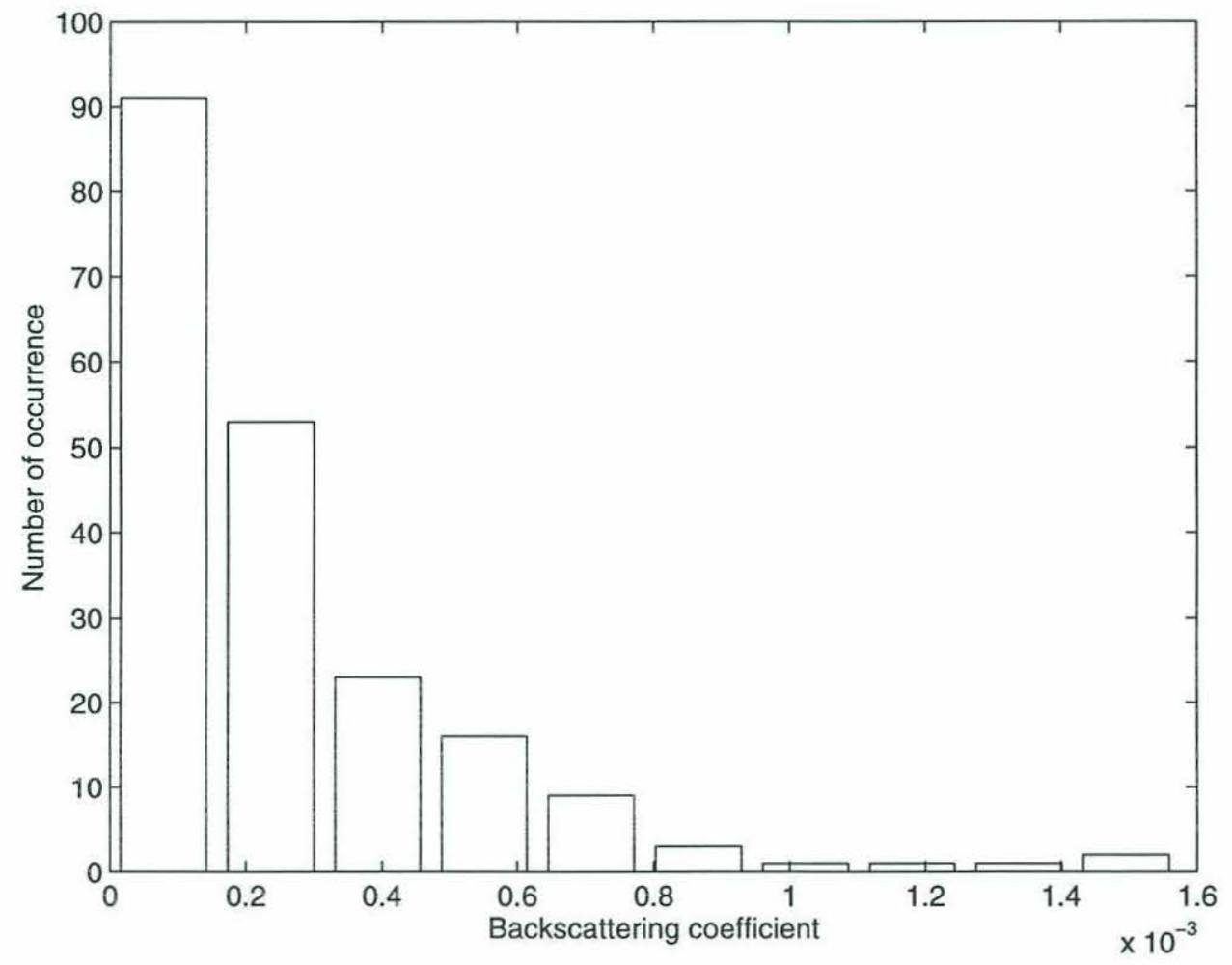

Figure B-1: Histogram of the simulated backscattering coefficients at $\theta=42$ degrees and $f=500 \mathrm{~Hz}$ (200 realizations). 


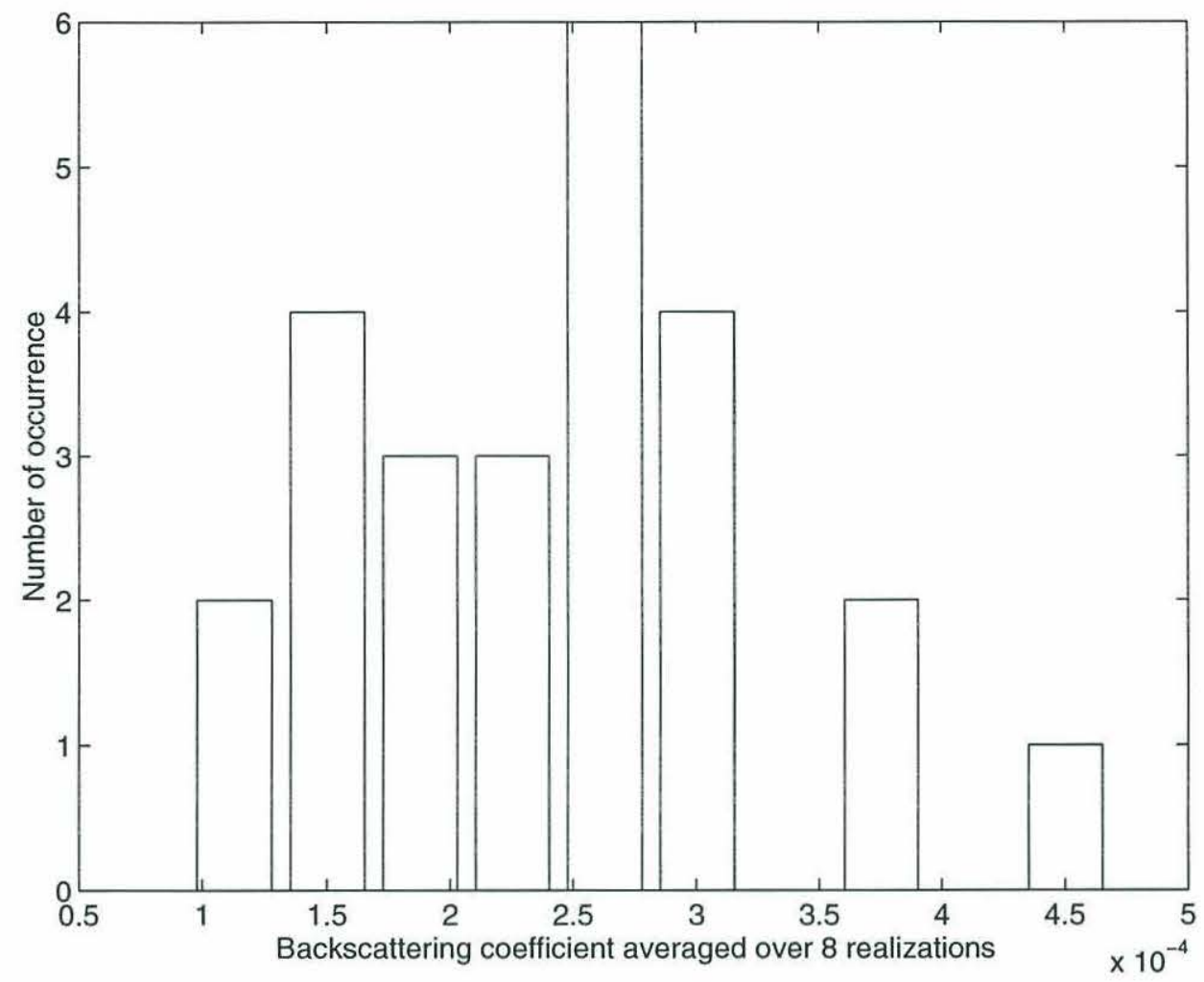

Figure B-2: Histogram of the simulated backscattering coefficients averaged over 8 realizations at $\theta=42$ degrees and $f=500 \mathrm{~Hz}$ (200 realizations).

able, we will arrive at a different random distribution. Assuming that the backscattering coefficient before averaging possesses an exponential distribution, the new random variable will have a Chi-square distribution with 16 degrees of freedom. In other words, it is closer to a Gaussian distribution than the original exponential distribution(it will be exactly a Gaussian distribution if the average is performed over an infinite number of independent realizations according to the Central Limit Theorem). This can be confirmed by the histogram of the simulated backscattering coefficient averaged over eight realizations as shown in Fig. B-2. As a result, the standard deviation estimated from the above equation would be a much better measurement of the dispersion, which is shown as the error bars in Fig. 6-13 and Fig. 6-14.

While for the data, only 8 pings are available. Therefore, the above procedure done to 200 realization of simulated backscattering coefficient cannot be performed, 


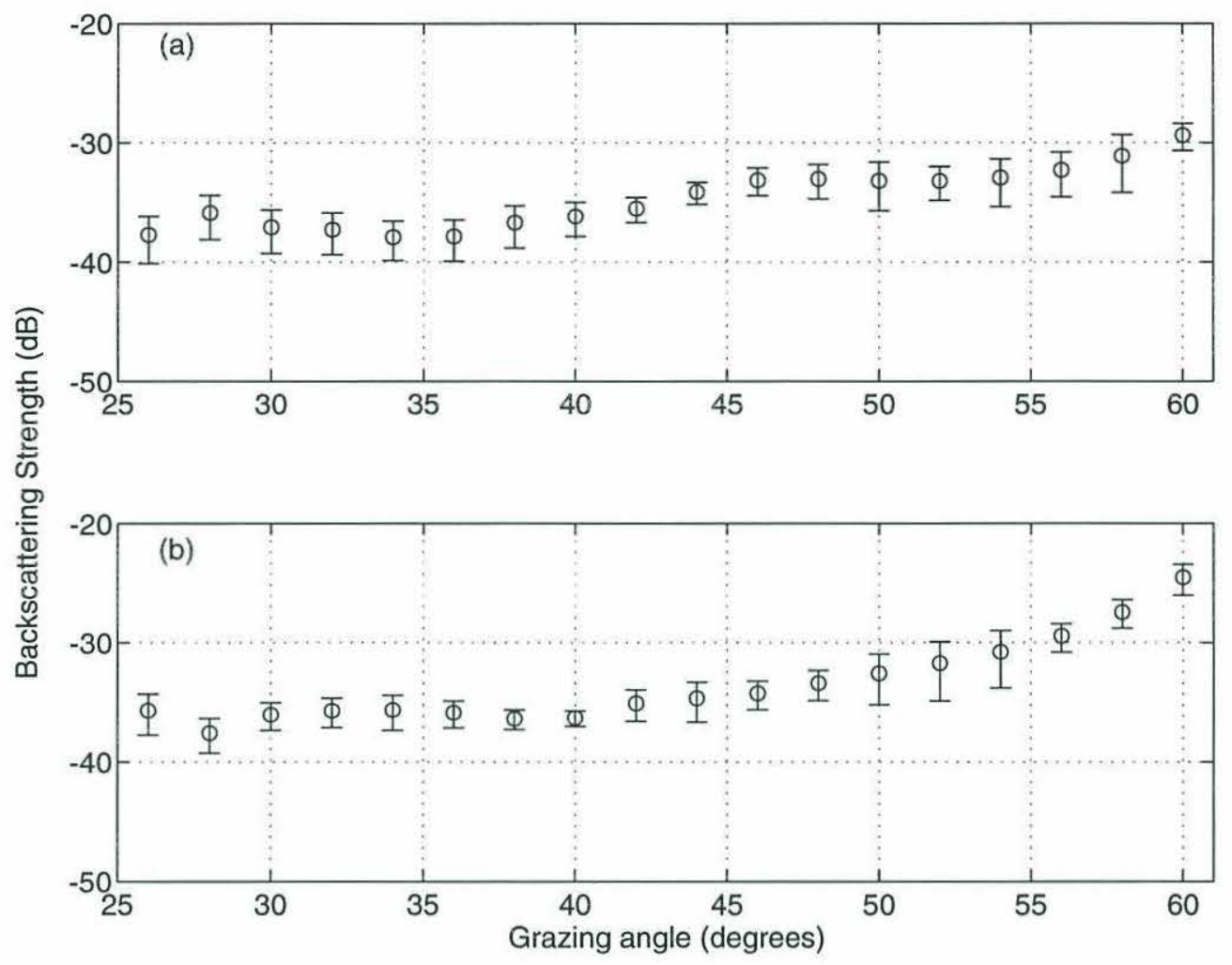

Figure B-3: The estimated backscattering strength with standard deviations (error bars) over all the frequencies for the (a) upper and (b) lower irregular layers at the east side of the sediment pond.

which is the reason why error bars are not plotted in Figs. 6-13 and 6-14 to represent the standard deviation estimated from the data. However, the average over independent frequencies in each ping should provide results which are similar to those obtained by averaging over pings. By using this approach, we can obtain reasonably good estimates of the standard deviation of the backscattering strength from the data, which are shown in Fig. B-3. 


\section{Bibliography}

[1] P. D. Mourad and D. R. Jackson. A model/data comparison for low-frequency bottom backscatter. J. Acoust. Soc. Am., 94:344-358, 1993.

[2] H. M. Merklinger. Bottom reverberation measured with explosive charges fired deep in the ocean. J. Acoust. Soc. Am., 44:508-513, 1968.

[3] D. R. Jackson, D. P. Winebrenner, and A. Ishimaru. Application of the composite roughness model to high-frequency bottom backscattering. J. Acoust. Soc. Am., 79:1410-1422, 1986.

[4] D. R. Jackson and K. B. Briggs. High-frequency bottom backscattering: roughness versus sediment volume scattering. J. Acoust. Soc. Am., 92:962-977, 1992.

[5] A. P. Lyons, A. L. Anderson, and F. S. Dwan. Acoustic scattering from the seafloor: Modeling and data comparison. J. Acoust. Soc. Am., 95:2441-2451, 1994.

[6] D. Tang, G. Jin, D. R. Jackson, and K. L. Williams. Analyses of high-frequency bottom and subbottom backscattering for two distinct shallow water environments. J. Acoust. Soc. Am., 96:2930-2936, 1994.

[7] B. H. Tracey. An Integrated Modal Approach to Surface and Volume Scattering in Ocean Acoustic Waveguides. PhD thesis, MIT/WHOI Joint Program, Cambridge, MA and Woods Hole, MA, 1996.

[8] D. Tang. Shallow water reverberation due to sediment volume inhomogeneities. IEEE J. Oceanic Eng., 1996. submitted. 
[9] D. Tang, G. V. Frisk, C. J. Sellers, and D. Li. Low-frequency acoustic backscattering by volumetric inhomogeneities in deep-ocean sediments. J. Acoust. Soc. Am., 98:508-516, 1995.

[10] D. Li. Low-frequency bottom backscattering data analysis using multiple constraints beamforming. Master's thesis, MIT/WHOI Joint Program, Cambridge, MA and Woods Hole, MA, 1995.

[11] J. S. Lim. Two-Dimensional Signal and Image Processing. Prentice-Hall, Englewood Cliffs, N.J., 1990.

[12] B. E. Tucholke, Private Communication.

[13] J. Jeffrey and P. M. Ogden. Bottom scattering strength measured in deep and shallow water using deep towed acoustics/geophysics system. NRL/FR/714097-9842, 1997. Naval Research Lab.

[14] P. C. Hines. Theoretical model of acoustic backscatter from a smooth seabed. J. Acoust. Soc. Am., 88:324-334, 1990.

[15] A. N. Ivakin. Sound scattering by random inhomogeneities of stratified ocean sediments. Sov. Phys. Acoust., 32:492-496, 1986.

[16] H. Schmidt. SAFARI: Seismo-acoustic fast field algorithm for range independent environments. User's Guide. Rep. SR-113, 1988. SACLANT Undersea Research Centre, La Spezia, Italy.

[17] P. M. Morse and K. U. Ingard. Theoretial Acoustics. McGraw-Hill,New York, 1968.

[18] D. R. Jackson, K. B. Briggs, K. L. Williams, and M. D. Richardson. Tests of models for high-frequency sea-floor backscatter. J. Acoust. Soc. Am., 1996. in press.

[19] M. B. Priestly. Spectral Analysis and Time Series. Academic, New York, 1981. 
[20] J. A. Ogilvy. Theory of Wave Scattering from Random Rough Surfaces. Adam Hilger, Bristol, 1991.

[21] J. H. Stockhausen. Scattering from the volume of an inhomogeneous half-space. Naval Research Establishment report,63/9, 1963. Canada.

[22] P. M. Morse. Vibration and Sound. McGraw-Hill, New York, 1948.

[23] A. W. Nolle, W. A. Hoyerand, J. F. Mifsud, W. R. Runyan, and M. B. Ward. Acoustic properties of water-filled sands. J. Acoust. Soc. Am., 35:1394-1408, 1963.

[24] P. A. Crowther. Some statistics of the sea-bed and acoustic scattering therefrom. In N. G. Pace, editor, Acoustics and the Sea-Bed, pages 147-155. Bath Univ., Bath,England, 1983.

[25] E. Y. Kuo. Wave scattering and transmission at irregular surfaces. J. Acoust. Soc. Am., 36:2135-2142, 1964.

[26] Yu. Yu. Zhitkovskii. Comparison of the sharp-pulse (explosion) and cw-burst methods for measuring the intensity of sound scattering by the ocean bottom. In Eighth All-Union Acoustics Conf., page 88, 1973. in Russian.

[27] V. I. Volovov and Yu. Yu. Zhitkovskii. Reflection and scattering of sound by the ocean bottom. In L. M. Brekhovskikh, editor, Ocean Acoustics, page 395. Nauka, Moscow, 1974. Part 6, in Russian.

[28] A. N. Ivakin and Yu. P. Lysanov. Theory of underwater sound scattering by random inhomogeneities of the bottom. Sov. Phys. Acoust., 27:61-64, 1981.

[29] Yu. P. Lysanov. Geoacoustic model of the upper sedimentary layer in shallow seas. Dokl. Adad. Nauk SSSR, 251:714, 1980.

[30] A. N. Ivakin and Yu. P. Lysanov. Underwater sound scattering by volume inhomogeneities of a bottom bounded by a rough surface. Sov. Phys. Acoust., 27:212-215, 1981. 
[31] P. D. Mourad and D. R. Jackson. High frequency sonar equation models for bottom backscatter and forward loss. In Oceans ' 89 Proceedings,IEEE, pages 1168-1175, New York, 1989.

[32] E. L. Hamilton. Compressional wave attenuation in marine sediments. Geophysics, 37:620-646, 1972.

[33] L. A. Chernov. Wave Propagation in a Random Medium. McGraw-Hill, New York, 1975. Translated from Russian by R. A. Silverman.

[34] P. C. Hines. Theoretical model of in-plane scatter from a smooth sediment seabed. J. Acoust. Soc. Am., 99:836-844, 1996.

[35] D. Tang. Acoustic Wave Scattering from a Random Ocean Bottom. PhD thesis, MIT/WHOI Joint Program, Cambridge, MA and Woods Hole, MA, 1991.

[36] D. Tang and G. V. Frisk. Plane-wave reflection from a random fluid half-space. J. Acoust. Soc. Am., 90:2751-2756, 1991.

[37] D. Tang and G. V. Frisk. Spectral parameterization of sound scattering from a random ocean bottom. J. Acoust. Soc. Am., 92:2792-2799, 1992.

[38] D. Tang and G. V. Frisk. Spatial correlation of acoustic waves scattered from a random ocean bottom. J. Acoust. Soc. Am., 97:2783-2803, 1995.

[39] T. Yamamoto. Acoustic scattering in the ocean from velocity and density fluctuations in the sediments. J. Acoust. Soc. Am., 99:866-879, 1996.

[40] D. D. Ellis. A shallow-water normal-mode reverberation model. J. Acoust. Soc. Am., 97:2804-2814, 1995.

[41] W. A. Kuperman and H. Schmidt. Self-consistent perturbation approach to rough surface scattering in stratified elastic media. J. Acoust. Soc. Am., 86:15111522, October 1989. 
[42] J.-Y. Liu, H. Schmidt, and W. A. Kuperman. Effect of a rough seabed on the spectral composition of deep ocean infrasonic ambient noise. J. Acoust. Soc. Am., 93:753-769, 1993.

[43] K. D. LePage. Elastic Scattering in Oceanic Waveguides. PhD thesis, Massachusetts Institute of Technology, 1993.

[44] H. Schmidt and W. A. Kuperman. Spectral respresentations of rough interface reverberation in stratified ocean waveguides. J. Acoust. Soc. Am., 97:2199-2209, April 1995.

[45] R. Lim. Multiple scattering by many bounded obstacles in a multilayered acoustic medium. J. Acoust. Soc. Am., 92:1593-1612, October 1991.

[46] R. H. Hackman and G. S. Sammelmann. Acoustic scattering in an inhomogeneous waveguide: Theory. J. Acoust. Soc. Am., 80:1447-1458, November 1986.

[47] P. Gerstoft and H. Schmidt. A boundary element approach to ocean seismoacoustic facet reverberation. J. Acoust. Soc. Am., 89:1629-1642, April 1991.

[48] K. V. Mackenzie. Bottom reverberation for 530- and 1030-cps sound in deep water. J. Acoust. Soc. Am., 33:1498-1504, 1961.

[49] H. Boehme, N. P. Chotiros, L. D. Rolleigh, S. P. Pitt, A. L. Garcia, T. G. Goldsberry, and R. A. Lamb. Acoustic backscattering at low grazing angles from the ocean bottom. Part I. Bottom backscattering strength. J. Acoust. Soc. Am., 77:962-974, 1984.

[50] J. R. Preston and T. Akal. Analysis of backscattering data in the Tyrrhenian sea. J. Acoust. Soc. Am., 87:119-134, 1989.

[51] P. C. Hines and P. J. Barry. Measurements of acoustic backscatter from the Sohm Abyssal Plain. J. Acoust. Soc. Am., 92:315-323, 1992.

[52] R. J. Urick. Principles of Underwater Sound. McGraw-Hill, New York, 1983. 
[53] T. Yamamoto. Acoustic scattering in the ocean from velocity and density fluctuations in the sediments. J. Acoust. Soc. Am., 99:866-879, 1996.

[54] E. K. Westwood. Complex ray methods for acoustic interaction at a fluid-fluid interface. J. Acoust. Soc. Am., 85:1872-1884, 1989.

[55] P. M. Morse and H. Feshbach. Method of Theoretical Physics. McGraw-Hill,New York, 1953.

[56] George V. Frisk. Ocean and Seabed Acoustics: A Theory of Wave Propagation. Prentice-Hall, Englewood Cliff, NJ, 1994.

[57] F. B. Jensen, W. A. Kuperman, M. B. Porter, and H. Schmidt. Computational Ocean Acoustics. American Institute of Physics, 1993.

[58] E. L. Hamilton. Sound velocity and related properties of marine sediments, North Pacific. Journal of Geophysics Research, 75:4423-4446, 1970.

[59] A. C. Kibblewhite. Attenuation of sound in marine sediments: A review with emphasis on new low-frequency data. J. Acoust. Soc. Am., 86:716-738, 1989.

[60] R. D. Stoll. Sediment Acoustics. Springer-Verlag, New York, 1989.

[61] A. K. Rogers, T. Yamamoto, and W. Carey. Experimental investigation of sediment effect on acoustic wave propagation in the shallow ocean. J. Acoust. Soc. Am., 93:1747-1761, 1993.

[62] E. L. Hamilton. Sound velocity gradients in marine sediments. J. Acoust. Soc. Am., 65:909-922, 1979.

[63] A. Frankel and R. W. Clayton. Finite difference simulations of seismic scattering: Implications for the propagation of short-period seismic waves in the crust and models of crustal heterogeneity. J. Geophysical Research, 91:6465-6489, 1986.

[64] T. Yamamoto. Velocity variabilities and other physical properties of marine sediments measured by crosswell acoustic tomography. J. Acoust. Soc. Am., 98:2235-2248, 1995. 
[65] V. I. Tatarski. Wave Propagation in a Turbulent Medium. McGraw-Hill,New York, 1961.

[66] J. A. Goff and T. H. Jordan. Stochastic modeling of seafloor morphology: Inversion of Sea Beam data for second-order statistics. Journal of Geophysics Research, 93:13589-13608, 1988.

[67] E. I. Thorsos. The validity of the Kirchhoff approximation for rough surface scattering using a Gaussian roughness spectrum. J. Acoust. Soc. Am., 83:78-92, 1987.

[68] H. Fan. Wave Theory Modeling of Three-Dimensional Seismo-Acoustic Reverberation in Ocean Waveguides. PhD thesis, MIT Ocean Engineering, Cambridge, MA, 1995.

[69] A. Mantoglou and J. L. Wilson. The turning bands method for the simulation of random fields using line generation by a spectral method. Water Resource Research, 18:1379-1394, 1982.

[70] M. J. Robin, A. L. Gutjhar, E. A. Sudicky, and J. L. Wilson. Cross-correlated random field generation with the direct Fourier transform method. Water Resource Research, 29:2385-2397, 1993.

[71] F. Ruan and D. McLaughlin. An efficient multivariate random field generator using the Fast Fourier Transform. Advances in Water Resource, 1996. submitted.

[72] J. S. Bendat and A. G. Piersol. Random Data: Analysis and Measurement Procedures. Wiley-Interscience, 1971.

[73] F. R. DiNapoli and R. L. Deavenport. Theoretical and numerical Green's function solution in a plane layered medium. J. Acoust. Soc. Am., 67:92-105, 1980.

[74] M. Abramowitz and I. A. Stegun. Handbook of Mathematical Functions: with Formulas, Graphs, and Mathematical Tables. Dover, New York, 1972. 
[75] M. B. Porter. The KRAKEN normal mode program. Rep. SM-245, 1991. SACLANT Undersea Research Centre, La Spezia, Italy.

[76] D. Tang, W. C. Burgess, S. D. Rajan, C. J. Sellers, G. V. Frisk, and D. Li. A summary of the direct-path scattering experiments in the ARSRP sediment pond and some preliminary results. In Fall ARSRP Research Symposium, December 1993. San Diego.

[77] S. Fu, R. Wilkens, and L. Frazer. Acoustic lance: New in situ seafloor velocity profiles. J. Acoust. Soc. Am., pages 234-242, 1996.

[78] W. K. Stewart, Private Communication.

[79] J. F. Gettrust, J. H. Ross, and M. M. Rowe. Development of a low frequency, deep tow geoacoustics system. Sea Technology, September 1991. 